\title{
Photonic applications based on bimodal interferometry in periodic integrated waveguides
}

\section{Luis Torrijos Morán}

Supervisor: Prof. Dr. Jaime García Rupérez

Doctoral Thesis

submitted for the degree of

Doctor of Philosophy in Telecommunication Engineering

July 2021, Valencia 


\section{Luis Torrijos Morán}

luitorm2@ntc.upv.es; luis.torrijosmoran@gmail.com

Photonic applications based on bimodal interferometry in periodic integrated waveguides

PhD Thesis, Nanophotonics Technology Center

UNIVERSITAT POLITÈCNICA DE VALÈNCIA

Cover: Luis Torrijos Morán

Render: Luis Torrijos Morán

First printing, July 2021 
A mis padres Luis y Teresa, a mi hermano Álvaro y a Andrea 

"Study hard what interests you the most in the most undisciplined, irreverent and original manner possible."

— Richard P. Feynman 



\section{Acknowledgements}

I want to express my gratitude to those who have been part of this period of my life full of learning and growth. Specially to my supervisor Jaime, who gave me the opportunity and freedom to make this thesis and develop my ideas. Thanks for your valuable guidance and encouragement, this work could not have been possible without your dedication. I would like to extend these acknowledgements to my colleagues of the Biophotonics group, Xisco, Raffaele, Ángela, David, Paula, Salva and Maribel, thanks for sharing all these experiences together and for your support. Thanks also to my colleagues from Ghent, especially Wim and Lukas, for welcoming me so warmly and get involved in the team despite the COVID circumstances.

I would also like to thank all the people from the NTC that have assisted me during all these years. Thanks to Amadeu, Jose, David, Laurent and Dora for helping me so much with the experimental work, and to Pablo and Antoine, for your assistance and guidance in some parts of the thesis. I want also to acknowledge my colleagues of the open room that have enlivened my stay at the NTC even more, Miguel, Gustavo, Manel, Javi, Elena, Sergio, Carles, Leo, thank you for making things easier and more fun for me.

I do not want to forget a special handful of people that I met at the NTC and who have accompanied me during all these years. Thanks for inspiring me and for giving me your insightful vision of life. They have been very enriching years, I learned a lot from all of you Fran, Jad, Javi, Kike and Seppo.

Por último, me gustaría dedicar un enorme agradecimiento a mi familia. En especial, a mi hermano Álvaro y a mis padres Luis y Teresa, porque, con todo su esfuerzo y dedicación, ellos son los verdaderos responsables de la persona que soy hoy en día. Y a ti, Andrea, por haber compartido conmigo todos estos momentos y los que nos quedan por vivir. Por enseñarme a ser feliz, gracias. 
Financial support is also gratefully acknowledged through postdoctoral FPI grants from Universitat Politècnica de València and Generalitat Valenciana (PAID 01-18 and ACIF/2019/009). 


\section{Abstract}

Silicon photonics is a key emerging technology in next-generation communication networks and data centers interconnects, among others. Its success relies on the ability of using CMOS-compatible platforms for the integration of optical circuits into small devices for a large-scale production at low-cost. Within this field, integrated interferometers play a crucial role in the development of several on-chip photonic applications such as biological sensors, electro-optic modulators, all-optical switches, programmable circuits or LiDAR systems, among others. However, it is well known that optical interferometry usually requires very long interaction paths, which hinders its integration in highly compact footprints. To mitigate some of these size limitations, several approaches emerged including sophisticated materials or more complex structures, which, in principle, reduced the design area but at the expense of increasing fabrication process steps and cost.

This thesis aims at providing general solutions to the long-standing size problem typical of optical integrated interferometers, in order to enable the densely integration of silicon-based devices. To this end, we combine the benefits from both bimodal waveguides and periodic structures, in terms of high-performance operation and compactness to design single-channel interferometers in very reduced areas. More specifically, we investigate the dispersive effects that arise from subwavelength grating and photonic crystal structures for their implementation in different bimodal interferometric configurations. Furthermore, we demonstrate various potential applications such as sensors, modulators and switches in ultra-compact footprints of a few square microns. In general, this thesis proposes a new concept of integrated interferometer that addresses the size requirements of current photonics and open up new avenues for future bimodal-operation-based devices. 



\section{Resumen}

La fotónica de silicio es una tecnología emergente clave en redes de comunicación e interconexiones de centros de datos de nueva generación, entre otros. Su éxito se basa en la utilización de plataformas compatibles con la tecnología CMOS para la integración de circuitos ópticos en dispositivos pequeños para una producción a gran escala a bajo coste. Dentro de este campo, los interferómetros integrados juegan un papel crucial en el desarrollo de diversas aplicaciones fotónicas en un chip como sensores biológicos, moduladores electro-ópticos, conmutadores totalmente ópticos, circuitos programables o sistemas LiDAR, entre otros. Sin embargo, es bien sabido que la interferometría óptica suele requerir caminos de interacción muy largos, lo que dificulta su integración en espacios muy compactos. Para mitigar algunas de estas limitaciones de tamaño, surgieron varios enfoques, incluyendo materiales sofisticados o estructuras más complejas, que, en principio, redujeron el área de diseño pero a expensas de aumentar los pasos del proceso de fabricación y el coste.

Esta tesis tiene como objetivo proporcionar soluciones generales al problema de tamaño típico de los interferómetros ópticos integrados, con el fin de permitir la integración densa de dispositivos basados en silicio. Para ello, aunamos los beneficios tanto de las guías de onda bimodales como de las estructuras periódicas, en términos de la mejora del rendimiento y la posibilidad para diseñar interferómetros monocanal en áreas muy reducidas. Más específicamente, investigamos los efectos dispersivos que aparecen en estructuras menores a la longitud de onda y en las de cristal fotónico, para su implementación en diferentes configuraciones interferométricas bimodales. Además, demostramos varias aplicaciones potenciales como sensores, moduladores y conmutadores en tamaños ultra compactos de unas pocas micras cuadradas. En general, esta tesis propone un nuevo concepto de interferómetro integrado que aborda los requisitos de tamaño de la fotónica actual y abre nuevas vías para futuros dispositivos basados en funcionamiento bimodal. 



\section{Resum}

La fotònica de silici és una tecnologia emergent clau en xarxes de comunicació i interconnexions de centres de dades de nova generació, entre altres. El seu èxit es basa en la utilització de plataformes compatibles amb la tecnologia CMOS per a la integració de circuits òptics en dispositius diminuts per a una producció a gran escala a baix cost. Dins d'aquest camp, els interferòmetres integrats juguen un paper crucial en el desenvolupament de diverses aplicacions fotòniques en un xip com a sensors biològics, moduladors electro-òptics, commutadors totalment òptics, circuits programables o sistemes LiDAR, entre altres. No obstant això, és ben sabut que la interferometría òptica sol requerir camins d'interacció molt llargs, la qual cosa dificulta la seua integració en espais molt compactes. Per a mitigar algunes d'aquestes limitacions de grandària, van sorgir diversos enfocaments, incloent materials sofisticats o estructures més complexes, que, en principi, van reduir l'àrea de disseny però a costa d'augmentar els processos de fabricació i el cost.

Aquesta tesi té com a objectiu proporcionar solucions generals al problema de grandària típica dels interferòmetres òptics integrats, amb la finalitat de permetre la integració densa de dispositius basats en silici. Per a això, combinem els beneficis tant de les guies d'ones bimodals com de les estructures periòdiques, en termes de funcionament d'alt rendiment per a dissenyar interferòmetres monocanal compactes en àrees molt reduïdes. Més específicament, investiguem els efectes dispersius que apareixen en estructures menors a la longitud d'ona i en les de cristall fotònic, per a la seua implementació en diferents configuracions interferomètriques bimodals. A més, vam demostrar diverses aplicacions potencials com a sensors, moduladors i commutadors en grandàries ultres compactes d'unes poques micres cuadrades. En general, aquesta tesi proposa un nou concepte d'interferòmetre integrat que aborda els requisits de grandària de la fotònica actual i obri noves vies per a futurs dispositius basats en funcionament bimodal. 



\section{Table of contents}

Acknowledgements $\quad$ v

Abstract vii

$\begin{array}{ll}\text { Resumen } & \text { ix }\end{array}$

Resum $\quad$ xi

1 Introduction 1

1.1 Objectives ......................... 4

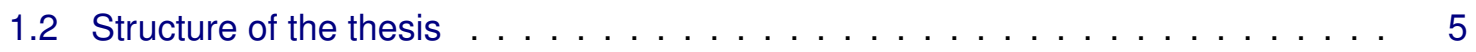

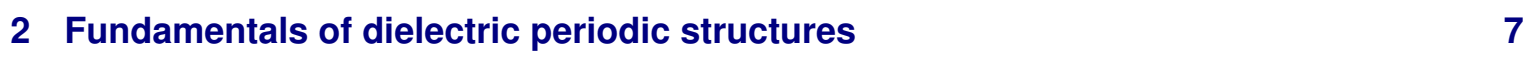

2.1 Introduction to optical waveguides $\ldots \ldots \ldots \ldots \ldots$

2.1 .1 Ray optics approach $\ldots \ldots \ldots \ldots \ldots \ldots \ldots$

2.1.2 Wave equation in electromagnetic theory . . . . . . . . . . . 11

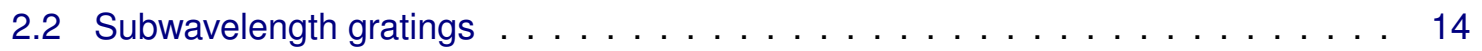

2.2.1 Electromagnetic waves in finely layered media . . . . . . . . . . . . 15

2.2.2 Waveguiding in subwavelength structures $\ldots \ldots \ldots \ldots$

2.2 .3 Multimode operation . . . . . . . . . . . . . . . . . . . 26

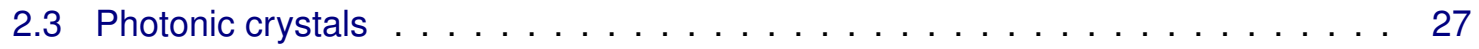

2.3.1 Eigenvalue problem of electromagnetic waves . . . . . . . . . . . . 28

2.3.2 Band structure analysis . . . . . . . . . . . . . . . . . . . . . 31

2.3.3 Photonic bandgaps and slow light phenomena . . . . . . . . . . . 34

2.3.4 One-dimensional periodic waveguides $\ldots \ldots \ldots \ldots \ldots$ 


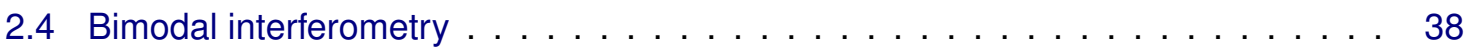

2.4.1 Integrated interferometers . . . . . . . . . . . . . . . . . 39

2.4.2 Bimodal behavior in periodic media . . . . . . . . . . . . . 41

3 Subwavelength grating bimodal waveguides $\quad 47$

3.1 Paper A: single-channel bimodal interferometric sensor using subwavelength structures . . . . . . . . . . . . . . . . . . . . . . 49

3.1 .1 Introduction . . . . . . . . . . . . . . . . 51

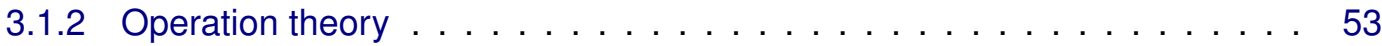

3.1 .3 Sensitivity analysis . . . . . . . . . . . . . . . . . . . 54

3.1 .4 Spectral analysis . . . . . . . . . . . . . . . . . . . 60

3.1 .5 Conclusions . . . . . . . . . . . . . . . . . . . . . . 64

3.1 .6 References . . . . . . . . . . . . . . . . . . . 65

3.2 Paper B: experimental study of subwavelength grating bimodal waveguides as ultrasensitive interferometric sensors . . . . . . . . . . . . . . . . . 69

3.2.1 Introduction . . . . . . . . . . . . . . . . . . . . 71

3.2 .2 Results . . . . . . . . . . . . . . . . . . . . . . . . . 72

3.2 .3 Conclusions . . . . . . . . . . . . . . . . . . . . 78

3.2 .4 References . . . . . . . . . . . . . . . . . . . . . . . 79

4 Bimodal interferometers in one-dimensional photonic crystals 83

4.1 Paper C: slow light bimodal interferometry in one-dimensional photonic crystal

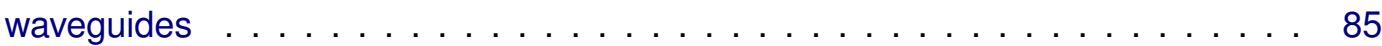

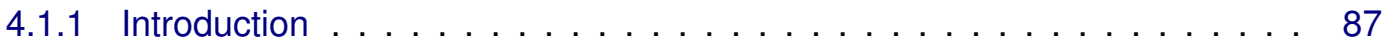

4.1 .2 Results . . . . . . . . . . . . . . . . . . . . . . . . 90

4.1 .3 Discussion . . . . . . . . . . . . . . . . . . . . . . . . . . . . . 104

4.1 .4 Materials and methods . . . . . . . . . . . . . . . . . . . 105

4.1 .5 References . . . . . . . . . . . . . . . . . . . . . . . . . 106

4.2 Paper D: ultra-compact optical switches using slow light bimodal silicon waveguides 111

4.2 .1 Introduction . . . . . . . . . . . . . . . . . . 113

4.2 .2 Concept and design . . . . . . . . . . . . . . . . . . . 115

4.2.3 Fabrication and characterization of the chip . . . . . . . . . . . . 119 
4.2 .4 Experimental demonstration $\ldots \ldots \ldots \ldots \ldots$

4.2 .5 Conclusion . . . . . . . . . . . . . . . . . . . . 123

4.2 .6 References . . . . . . . . . . . . . . . . . . . . . . . . . . 125

4.3 Paper E: design of slow-light-enhanced bimodal interferometers using dimensionality reduction techniques . . . . . . . . . . . . . . . . . . 129

4.3 .1 Introduction . . . . . . . . . . . . . . . . . . . . 131

4.3 .2 Figure of merit definition . . . . . . . . . . . . . . . . . . 133

4.3 .3 Design optimization . . . . . . . . . . . . . . . . . . . . . . . . 134

4.3.4 Comparison of the results and discussion $\ldots \ldots$. . . . . . . . . 140

4.3 .5 Conclusions . . . . . . . . . . . . . . . . . . . . . . . . . . . . 142

4.3 .6 References . . . . . . . . . . . . . . . . . . . . . . . . . . 144

5 Discussion and conclusions 151

5.1 General discussion of the results . . . . . . . . . . . . . . . . . . . 151

5.1 .1 Bimodal subwavelength waveguides $\ldots \ldots \ldots \ldots \ldots$

5.1 .2 Bimodal 1D photonic crystals . . . . . . . . . . . . . . . . . . . . 154

5.2 Main conclusions . . . . . . . . . . . . . . . . . . . . . . . . . 157

5.3 Future work . . . . . . . . . . . . . . . . . . . 160

5.4 Concluding remarks $\ldots \ldots \ldots \ldots \ldots \ldots \ldots$

$\begin{array}{ll}\text { Author's merits } & 163\end{array}$

$\begin{array}{ll}\text { Bibliography } & 167\end{array}$

$\begin{array}{ll}\text { List of figures } & 181\end{array}$

$\begin{array}{ll}\text { List of acronyms } & 189\end{array}$ 



\section{Chapter 1}

\section{Introduction}

Since the discovery of the transistor in 1947, the miniaturization of electronic components in limited space has stimulated considerable research effort from both academia and industry. In 1965, Gordon Moore published his famous essay, the so-called Moore's law, in which he predicted that the number of transistors on a chip would double every two years [1]. This fact established a timeline that came to dominate the information-technology revolution in the following decades that still prevails today (see Fig. 1.1). However, Moore's prediction seems to be nearing to its end. Transistor size is reaching the nanometer-limit, in which quantum uncertainties appear and thus the transistor performance becomes undependable [2].

Despite this, innovation beyond Moore's law continues by bringing together different technologies that overcome some of these electronic limitations. This is the case of photonics, concerning the manipulation of light, that provides potential solutions to mitigate the electronics bottleneck in terms of energy efficiency and bandwidth [3, 4]. In this context, silicon photonics arises as a disruptive technology driven by the needs of datacom and telecom, with significant implications in many different areas such as data centers interconnects [5-7], lab-on-a-chip (LoC) platforms [8-10] or high-performance computing devices [11, 12], among others. The success of silicon photonics stems from its ability to use complementary metal-oxide-semiconductor (CMOS) foundries for high-volume production of photonic integrated circuits (PICs) at low cost. The mature CMOS industry makes silicon photonics very well positioned to become dominant against other technologies, which explains the vast increase in scientific interest over the years [13]. The potential of silicon photonics was first investigated in 1985 [14], where high index contrast waveguides were fabricated on silicon-on-insulator (SOI) wafers. Since then, both passive and active components have been extensively demonstrated for the near-infrared regime by using standard CMOS fabrication processes [15, 16]. 


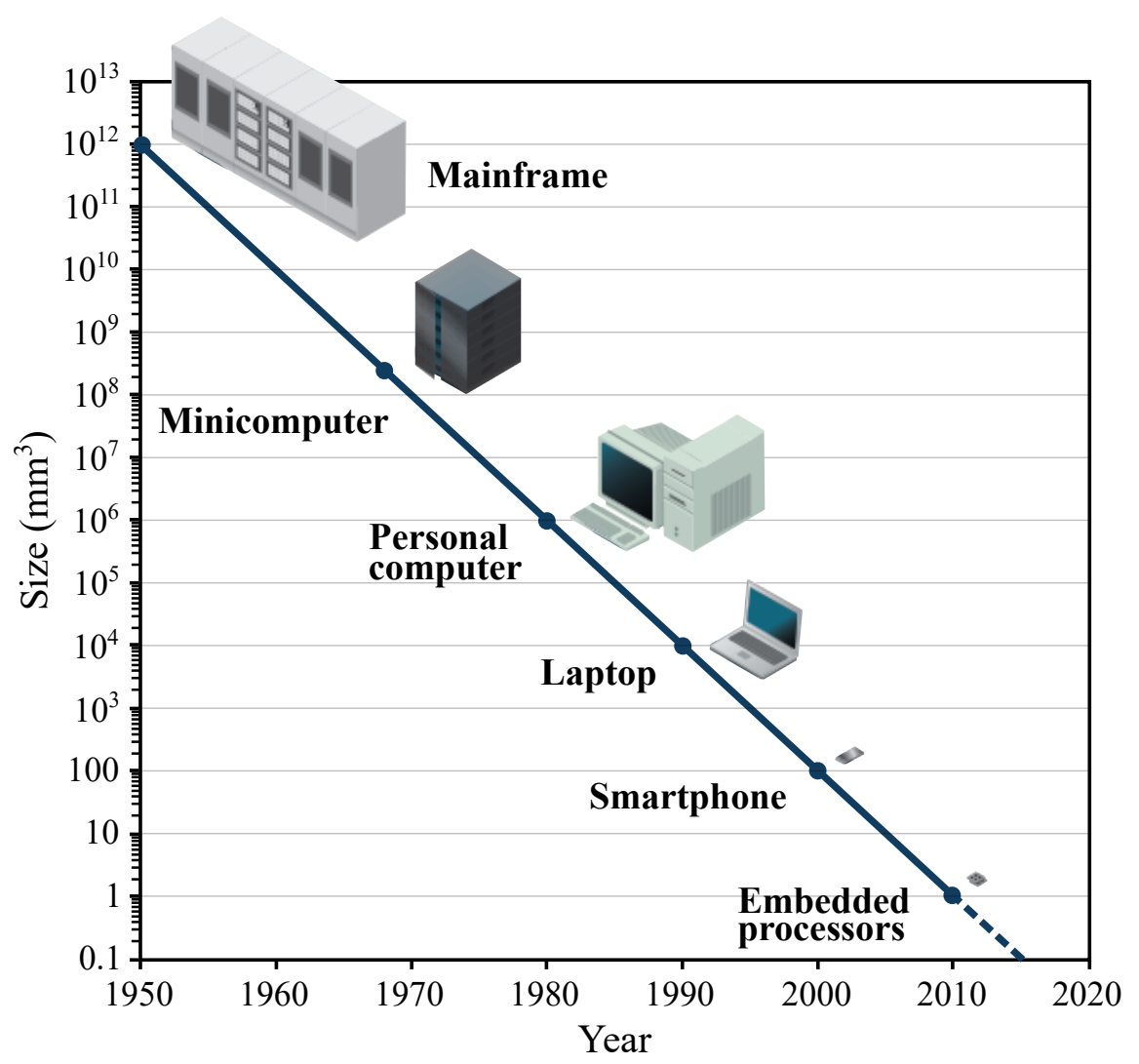

Fig. 1.1 Electronic equipment shrinkage in size as a function of time. Approximately, every decade a new class of machines emerged in agreement with Moore's law prediction [2].

Likewise, engineering novel wavelength-scale structures with multiple photonic functions have shown an unprecedented miniaturization in the final device size, which in turn reduces costs through high-yield wafer-scale processes [17]. On this matter, integrated interferometers emerged as key building blocks in all types of photonic devices such us modulators, switches and sensors [18-20]. Mach-Zehnder interferometers (MZIs) are the most commonly used structures therein to perform optical interferometry in an integrated circuit. Here light is split into two different paths and recombined afterwards to create an interference pattern at the output, which shifts for induced changes in the relative phase. Nowadays, MZI-based structures are reaching a degree of maturity never imagined, offering remarkable improvements for low-power consumption and high-performance operation [21, 22]. Nevertheless, conventional MZIs are usually too large, which hinders the fabrication and miniaturization of densely PICs. Many of these components have a length scale of millimeters and even centimeters in order to enhance the accumulated phase shift between both optical paths at the output [23, 24].

Thereafter, novel structures are reported aimed at reducing the resulting footprint, for instance, by inserting periodic structures in the MZI arms to increase light-matter 
interaction [25]. These are the so-called subwavelength grating (SWG) waveguides, consisting of periodically patterned structures arranged in the propagation direction of light and whose lattice constant is smaller than the effective wavelength so that refraction effects are suppressed [26, 27]. On the other hand, if the periodicity is in the order of the effective wavelength of light a Bragg mirror is obtained, where new phenomena appear due to induced reflections in the structure. This is the case of the well-known photonic crystals (PhCs) [28], where light may be slowed down, thereby reducing the physical interferometers length while the optical path remains large. Indeed, notable improvement is obtained by combining them with MZI-based configurations to develop highly compact devices $[29,30]$. In all aforementioned approaches, although a significant reduction in the device size is reached, classical MZI configurations still require additional photonic structures such as power splitters and different optical waveguides to perform the interference. In this sense, bimodal (BiM) waveguides address existing MZI size limitations as they do not need any extra structures to make two optical signals interfere $[31,32]$. The operation principle of BiM approaches is based on the interference between two electromagnetic modes supported by the same optical waveguide, so that the entire process is carried out in a single-channel structure. Most of these BiM waveguides have been widely demonstrated in silicon nitride platforms as evanescent-wave optical sensors for the detection of various biological substances [33-35]. These type of BiM interferometers present a highly reduced lateral size compared to MZIs, although they still suffer from very long optical waveguides of several centimeters.

It seems fairly evident that there is still a lack of novel approaches that fully address the long-standing challenge of miniaturization of photonic integrated interferometers. This thesis aims to provide general solutions to this field by changing the classical standpoint of on-chip optical interferometry. Having this in mind, a new concept of interferometer consisting of periodically patterned materials in all-dielectric single-channel structures is proposed. This approach encompasses the benefits from periodic structures and BiM waveguides to design high-performance optical interferometers in extremely reduced footprints. The potential of these designs is demonstrated for a wide range of applications such as refractive index (RI) sensors, modulators and switches with significant improvements compared to conventional solutions. Overall, this dissertation outlines the ever-growing demand of more powerful photonic devices in next-generation communication systems where the densely integration of optical circuits at the chip level is of great interest. Here we pave the road towards the realization of a new interferometric configuration which is not intended to supersede existing ones but complement them to strengthen silicon photonics against competing technologies. 


\subsection{Objectives}

Given the number of applications based on novel structures that have arisen in photonics over the last years, this thesis attempts to contribute to the field of optical integrated circuits by engineering new interferometric configurations. To this end, the benefits from both bimodal waveguides and periodic dielectric structures are encompassed in order to reduce the design area as much as possible and facilitate their CMOS-compatible integration in optical circuitry for mass level production.

In this context, the main goal is to investigate the bimodal behavior of light in periodic waveguides and how we can benefit from the resulting effects to develop interferometric applications. In such a scenario, the relationship between the wavelength of light and the structure periodicity will determine the operating regime and thus the physical modeling that must be considered in the design process. Specifically, we will look into detail subwavelength grating and photonic crystal structures as different bimodal dispersion effects are obtained using each of them. Specifically, the main objectives of employing these two types of periodic structures are:

- Develop a bimodal dispersion engineered waveguide in a SWG structure. By using these types of periodic waveguides, the device is intended to support a fundamental dispersive mode that will produce a flat phase shift as a function of wavelength. This fact enables high-performance spectral-based refractive index sensors, whose sensitivity does not depend on the sensor length so that very short interferometric schemes can be conceived.

- Develop a bimodal PhC waveguide working in the slow light regime. By using PhCs, we drastically reduce the group velocity of the high-order mode which is the most sensitive to refractive index variations. As a result, we enhance the accumulated phase shift at the output without the need of very long interferometric paths. These designs are intended to be experimentally demonstrated for switching and sensing in very compact footprint devices.

Regarding the methodology, the design starts from a semi-analytical modeling to propose a device concept, which is later on numerically simulated to prove its performance. Herein, our objective is to devise, simulate and demonstrate photonic applications based on bimodal SWG and PhC waveguides. The requirements to accomplish this aim rely on a theoretical background and a proper use of the simulations tools, either commercial or open-source software. Thereafter, optimized designs are fabricated and characterized to obtain the measured results and compare them with the initial ideas. To this end, an adequate use of the institute's facilities, including the clean room and laboratories, is made to fabricate and carry out the experimental work. 


\subsection{Structure of the thesis}

This thesis describes the work presented to obtain the degree of Doctor of Philosophy $(\mathrm{PhD})$ in Telecommunication Engineering under the doctorate program of the Universitàt Politècnica de València (UPV). The research has been carried out in the Nanophotonics Technology Center of the UPV under the supervision of Dr. Jaime García-Rupérez and during a three-month research stay at the Photonics Research Group ${ }^{1}$ of the Ghent University under the guidance of Prof. Wim Bogaerts.

The organization of this dissertation is conceived as a collection of papers published in peer-reviewed scientific journals. In these articles we can find the author's original contributions with the main findings of this thesis ${ }^{2}$, as well as the corresponding state of the art. This document is subsequently structured in the following chapters and sections:

- Chapter 1: Introduction puts the work into the context of photonics. More specifically, the miniaturization of optical circuits and the contributions of this thesis to this field are discussed. The main goals of this dissertation and the procedures to achieve them are also detailed.

- Chapter 2: Fundamentals of dielectric periodic structures provides the principles and origins of light propagation through periodic media, specifically in subwavelength and photonic crystal structures. Firstly, light confinement within optical waveguides is introduced by using ray optics approach and Maxwell's formalism. The latter part describes the basis of integrated interferometry including bimodal approaches in periodic media.

- Chapter 3: Subwavelength grating bimodal waveguides introduces the context of SWGs and how these are used to develop novel photonic applications. It also includes our original works on the theoretical design and experimental demonstration of evanescent-wave based RI sensors on bimodal SWG structures, Paper A and Paper B, respectively.

- Chapter 4: Bimodal interferometers in one-dimensional photonic crystals includes the state of the art of PhCs in connection with optical interferometers and bimodal waveguides aimed at reducing the design length. Our original contributions to the field in devising and conceiving bimodal slow light interferometers as sensors, modulators and switches are presented in Paper C and Paper D. In Paper E we explore new slow-light-enhanced bimodal designs by using dimensionality reduction techniques.

\footnotetext{
${ }^{1}$ From September to December 2020.

2 The detailed list of publications is shown in the Author's merits section.
} 
- Chapter 5: General discussions of the results outlines the main findings of this thesis as well as discuss the degree of compliance of the objectives. Further steps are also provided to be addressed in future work related with the topics under discussion.

At the end of the document, a section with complete list of the Author's merits is given, including the main contributions to scientific journals and conferences. A section of References that have been cited throughout the previous sections are also listed in the appearance order in which they have been included. 


\section{Chapter 2}

\section{Fundamentals of dielectric periodic structures}

Since ancient times, progress of mankind is largely based on the ability of our ancestors to recognize the utility of natural materials from the earth. From the improvements of the mechanical properties in metallurgy, ceramics and plastics to the semiconductor revolution in electronics, many of the humanity advances stem from a deeper knowledge of materials and its interaction with the physical laws that govern nature.

In the last few decades, the manipulation of light has attracted increasing attention due to various prominent advances in optics such as the invention of the laser and the development of semiconductor optical devices and fabrication techniques [36-38]. In this context, the search of a suitable transmission medium that guide light became a key aspect in the progress of this new technology [39]. In the early stages, lens systems and mirrors were employed to build optical waveguides, although they were too expensive to use them massively in optical communications systems [40]. With the appearance of the glass optical fiber with losses below $10 \mathrm{~dB} / \mathrm{km}$ much of these problems were mitigated, and fibers became dominant far outweigh all its competitors [41]. In the following years, however, with the rapid growth of integrated optics, new breakthroughs emerged on optical circuits based on thin film technology [42], and thereby, dielectric optical waveguides on CMOS-compatible platforms were widely used in all types of integrated photonic applications due to its straightforward and low-cost implementation [43, 44].

In this chapter, we provide a comprehensive description on the fundamentals of light propagation through periodic waveguides. We first introduce general concepts of optical waveguiding in homogeneous media and then we study light in periodically patterned structures, specifically subwavelength gratings and photonic crystals, to develop common path interferometers in highly dispersive bimodal waveguides. 


\subsection{Introduction to optical waveguides}

Here we present a brief description of optical guiding through dielectric structures, by firstly introducing two-dimensional models for the case of symmetric and asymmetric slab waveguides and then considering fully three-dimensional rectangular ones. Light transmission from the point of view of both geometrical and wave optics is given, as well as the derivation of the resulting guided and leaky modes [45, 46].

\subsubsection{Ray optics approach}

Let us first introduce the well-known ray optical model to investigate simple phenomena in a symmetric planar waveguide. Consider an incident ray $E_{i}$ propagating through a medium with a refractive index $n_{1}$ and with a certain angle $\theta_{1}$, see Fig. 2.1. At the interface with the medium of refractive index $n_{2}$, light is partially reflected $E_{t}$ and transmitted $E_{r}$ with an angle $\theta_{2}$, following the Snell's law:

$$
n_{1} \sin \theta_{1}=n_{2} \sin \theta_{2}
$$

Since the refractive index of the core medium $n_{1}$ is higher than $n_{2}, \theta_{2}$ will be thereby greater than $\theta_{1}$. As a result, there is a critical angle from which all light rays will be reflected and none transmitted into the $n_{2}$ medium. This is known as total internal reflection (TIR) and the angle that defines this region is given by the following equation

$$
\sin \theta_{c}=\frac{n_{2}}{n_{1}}
$$

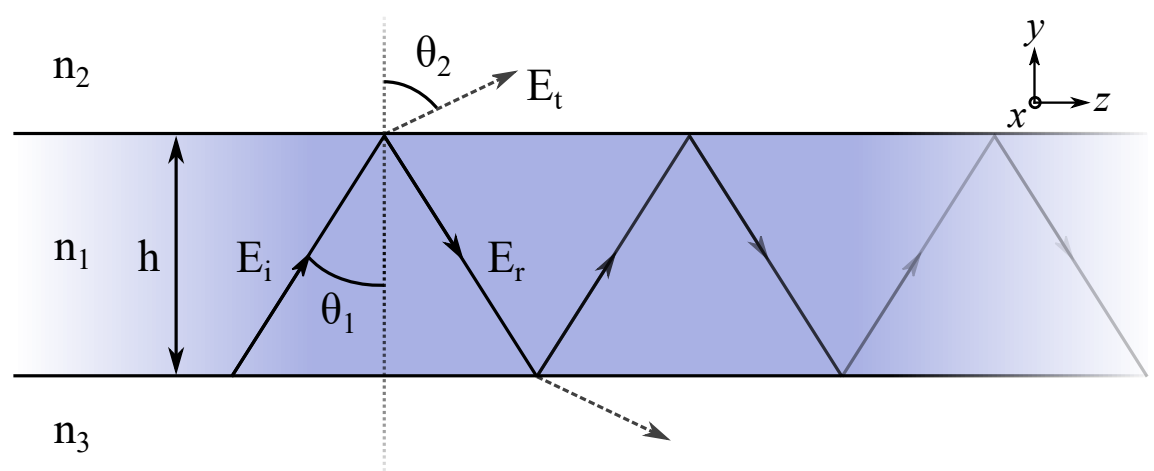

Fig. 2.1 Light rays propagating through a planar optical waveguide with three different media with a refractive index of the core $n_{1}$, and upper and lower cladding $n_{2}$ and $n_{3}$, respectively. 
If we now consider a third medium of refractive $n_{3}=n_{2}$ for which TIR is also produced, we can get an idea on how light propagates in the $z$ direction. However, not all angles greater than the critical one are supported in the optical waveguide. Fresnel formulas define that the incident and reflected rays are related as

$$
E_{r}=r E_{i}
$$

where $r$ is the complex reflection coefficient. Considering that rays are actually electromagnetic waves that bounce within the optical waveguide, we can define two polarizations: transverse electric (TE) with the electric field along the $x$ axis, and transverse magnetic (TM) with the electric field along the $y$ axis. For the TE polarization, the reflection coefficient is expressed as

$$
r_{T E}=\frac{n_{1} \cos \theta_{1}-n_{2} \cos \theta_{2}}{n_{1} \cos \theta_{1}+n_{2} \cos \theta_{2}}
$$

and for TM as

$$
r_{T M}=\frac{n_{2} \cos \theta_{1}-n_{1} \cos \theta_{2}}{n_{2} \cos \theta_{1}+n_{1} \cos \theta_{2}}
$$

using the Snell's law of Eq. 2.1.1 and substituting we obtain

$$
\begin{aligned}
& r_{T E}=\frac{n_{1} \cos \theta_{1}-\sqrt{n_{2}^{2}-n_{1}^{2} \sin ^{2} \theta_{1}}}{n_{1} \cos \theta_{1}+\sqrt{n_{2}^{2}-n_{1}^{2} \sin ^{2} \theta_{1}}}, \\
& r_{T M}=\frac{n_{2}^{2} \cos \theta_{1}-n_{1} \sqrt{n_{2}^{2}-n_{1}^{2} \sin ^{2} \theta_{1}}}{n_{2}^{2} \cos \theta_{1}+n_{1} \sqrt{n_{2}^{2}-n_{1}^{2} \sin ^{2} \theta_{1}}} .
\end{aligned}
$$

When the incident angle is greater than the critical one, the incident and reflected waves are equal, so that the reflection coefficient is $|r|=1$. Given that $r$ is a complex number and hence a phase shift is imposed, we can rewrite the reflection coefficient as $r=e^{j \phi}$. Assuming these considerations, we can express Eqs. 2.1.6 in terms of the phase shift for both TE and TM polarization as

$$
\begin{aligned}
& \phi_{T E}=2 \tan ^{-1} \frac{\sqrt{\sin ^{2} \theta_{1}-\frac{n_{2}^{2}}{n_{1}^{2}}}}{\cos \theta_{1}}, \\
& \phi_{T M}=2 \tan ^{-1} \frac{\sqrt{\frac{n_{1}^{2}}{n_{2}^{2}} \sin ^{2} \theta_{1}-1}}{\frac{n_{2}}{n_{1}} \cos \theta_{1}} .
\end{aligned}
$$


Extending the rays approach, light is composed by electromagnetic waves that follow the rays path. These waves can be written in terms of the phase shift in the exponential form for the electric and magnetic fields as follows

$$
\begin{aligned}
& E=E_{0} e^{j\left(k_{z} \pm \omega t\right)}, \\
& H=H_{0} e^{j\left(k_{z} \pm \omega t\right)},
\end{aligned}
$$

where $k_{z}$ is the wavector in the propagation direction, $\omega$ the angular frequency and $t$ the time. Let the waveguide height be $h$, so that is also confined in the $y$ direction as a standing wave. The phase shift of this wave across the waveguide and back again can be represented as

$$
\phi_{h}=2 h k_{y}=2 h k_{0} n_{1} \cos \theta_{1},
$$

where $k_{0}$ is the wavevector in free space. It is clear that reflections occur at the upper and lower interfaces of the waveguide, expressed in Eqs. 2.1.7. Let us call this phase shifts at $\phi_{u}$ and $\phi_{l}$, respectively, so that together with Eq. 2.1.9 the total phase shift in the waveguide is expressed as

$$
\phi_{t}=2 h k_{0} n_{1} \cos \theta_{1}-\phi_{u}-\phi_{l},
$$

where $\phi_{t}$ must an integer multiple times $2 \pi$ for the preservation of the wave propagation along the waveguide, thus obtaining

$$
2 h k_{0} n_{1} \cos \theta_{1}-\phi_{u}-\phi_{l}=2 m \pi
$$

Therefore, there will be a finite set of angles that satisfy Eq. 2.1.11, and hence a wavevector, or propagation constant that characterizes light propagation. Each solution is known as light modes and they are different depending on the polarization of light. The first solution $m=1$ for the TE polarization is known as the fundamental mode, and it is usually expressed as the $T E_{0}$ mode. Higher order modes are subsequently described by $m$ and the total number of modes is limited by the waveguide dimensions. In the case of the symmetrical planar waveguide, for $n_{2}=n_{3}$, the upper and lower phase shifts are equal $\phi_{u}=\phi_{l}$, so that substituting Eqs. 2.1.7 in Eq. 2.1.11, we obtain for the TE polarization

$$
2 h k_{0} n_{1} \cos \theta_{1}-4 \tan ^{-1}\left(\frac{\sqrt{\sin ^{2} \theta_{1}-\left(n_{2} / n_{1}\right)^{2}}}{\cos \theta_{1}}\right)=2 m \pi \text {. }
$$

In a asymmetric planar waveguide, where $n_{2} \neq n_{3}$, the upper and lower reflections do not produce the same phase shift so that $\phi_{u} \neq \phi_{l}$. In this case, Eq. 2.1.11 for the TE 
polarization yields

$$
k_{0} n_{1} \cos \theta_{1}-m \pi=\tan ^{-1}\left(\frac{\sqrt{\sin ^{2} \theta_{1}-\left(n_{2} / n_{1}\right)^{2}}}{\cos \theta_{1}}\right)+\tan ^{-1}\left(\frac{\sqrt{\sin ^{2} \theta_{1}-\left(n_{3} / n_{1}\right)^{2}}}{\cos \theta_{1}}\right) .
$$

These equations can be solved to find the angles $\theta_{1}$ for each mode $m$ that satisfy the accumulation of phase shifts previously imposed. Therefore, for a given wavelength, it is possible to have a negative square root value on the right-hand side of Eq. 2.1.13 so that there are no solutions. This could happen when the critical angle condition is not reached because the height $h$ is not large enough to support the fundamental mode. Such thickness will be different for each polarization. On the other hand, it is often convenient that the waveguide supports only the fundamental mode, single mode operation. This occurs when the wavelength is sufficiently small so that a single solution for the $m=0$ case is obtained from Eq. 2.1.13. This point, when the fundamental mode appears, is known as the cut-off frequency and determines from which wavelength light can propagate through the waveguide.

We can take the planar waveguide model a bit further to extract another interesting quantity. We can define the effective index of a certain mode and polarization as

$$
n_{e f f}=n_{1} \sin \theta_{1}
$$

so that it relates with the propagation constant as

$$
n_{e f f}=\beta \frac{\lambda_{0}}{2 \pi}
$$

The effective index is of particular interest as it characterizes a certain propagating mode in a specific waveguide. It is equivalent to think of a mode propagating straight in a waveguide with a refractive index $n_{e f f}$. It is also worth noting that the effective index value must be in between the refractive index of the core and the cladding, otherwise the resulting mode will not be propagated and it will be radiated out of the waveguide.

\subsubsection{Wave equation in electromagnetic theory}

A more complete study of an optical waveguide can be carried out by means of the Maxwell's equations, that relate the electric $\mathbf{E}$ and magnetic field $\mathbf{H}$ vectors. They are 
expressed in its differential form as ${ }^{1}$

$$
\begin{aligned}
& \nabla \cdot \mathbf{D}=\rho, \\
& \nabla \cdot \mathbf{B}=0, \\
& \nabla \times \mathbf{E}+\frac{\partial \mathbf{B}}{\partial t}=0, \\
& \nabla \times \mathbf{H}-\frac{\partial \mathbf{D}}{\partial t}=\mathbf{J},
\end{aligned}
$$

where $\rho$ is the electric charge and $\mathbf{J}$ the current density vector. The constitutive relations ${ }^{2}$ relate the $\mathbf{E}$ and $\mathbf{H}$ fields with the electric and magnetic flux density fields $\mathbf{D}$ and $\mathbf{B}$ in a lossless medium as

$$
\begin{aligned}
& \mathbf{D}=\epsilon_{r} \mathbf{E}, \\
& \mathbf{B}=\mu_{r} \mathbf{H},
\end{aligned}
$$

where $\epsilon_{r}$ and $\mu_{r}$ are the relative permittivity and permeability of the medium, respectively.

By properly operating with Eqs. 2.1.16 and 2.1.17 we obtain the wave equation that describes the propagation of electromagnetic waves, mathematically expressed as

$$
\nabla^{2} \mathbf{E}=\mu_{r} \epsilon_{r} \frac{\partial^{2} \mathbf{E}}{\partial t^{2}}
$$

In a planar waveguide, the wave equation can be simplified by considering that the electric field only exists in the $x$ direction, TE polarization. We also consider that the field is uniform in $x$ since it is assumed to be infinite in this direction. Taking this into account, we obtain the following scalar equation

$$
\frac{\partial^{2} E_{x}}{\partial^{2}}+\frac{\partial^{2} E_{x}}{\partial_{z}^{2}}=\mu_{r} \epsilon_{r} \frac{\partial^{2} E_{x}}{\partial t^{2}}
$$

Given that the electric field $\mathbf{E}$ only exists in the $x$ direction, we can express it in its exponential form as

$$
E_{x}=E(y) e^{-j(\beta z-\omega t)},
$$

so the wave equation is reduced to

$$
\frac{\partial^{2} E_{x}}{\partial y^{2}}+k_{y i}^{2} E_{x}=0
$$

\footnotetext{
${ }^{1}$ Bold variables represent vectorial magnitudes with several components.

${ }^{2} \mathrm{~A}$ more detailed derivation of the constitutive relations is explained in the photonic crystals section.
} 
whence

$$
k_{y i}^{2}=k_{0}^{2} n_{i}^{2}-\beta^{2},
$$

where $n_{i}$ and $k_{y i}$ can represent either the core or the upper and lower cladding. Therefore, the wave equation allow us to calculate the field profile of each mode, which was not possible by the optics ray approach. Solving the $x$ component of the electric field for the cladding, we obtain ${ }^{3}$

$$
E_{x}(y)=E_{\text {cladd }} \exp \left[-k_{y c l a d d}(y-h / 2)\right] \quad \text { for } \quad y \geq(h / 2)
$$

and for the core

$$
E_{x}(y)=E_{\text {core }} \exp \left[-j k_{y c o r e} y\right] \text { for } \quad-(h / 2) \leq y \leq(h / 2) .
$$

Applying boundary conditions of continuity on $E_{x}$ and its derivative we can also obtain the following expression for the propagation constants of the modes at each interface

$$
2 \tan ^{-1}\left(\frac{k_{y c l a d d}}{k_{y c o r e}}\right)=h k_{y c o r e}+m \pi \text {. }
$$

Substituting the value of each propagation constant into Eq. 2.1.25 we get the same expressions than the ones obtained for the optics ray approach, however, we now have the field profile equations for each mode $m$.

The planar model has allowed us to understand the guiding of light within an optical waveguide. However, more complex structures based on three-dimensional models with a finite dimension in the $x$ direction are the ones used in integrated optics. These are the rectangular waveguides, see Fig. 2.2, where light propagates along the $z$ direction and where the confinement is not only in the $y$ direction but also in the $x$ direction. The guiding mechanism, as in the case of planar examples, relies on TIR, given that the refractive index of the core is greater than in both claddings. Hence, we have to design not only the thickness, but also the width of the waveguide for the desired application. Rectangular waveguides are fabricated on planar substrates, giving rise to different types depending on the fabrication technology, materials and applications. In Fig. 2.2 we can see the three main rectangular waveguides examples with three different geometrical shapes. It is worth also noting that there are other aspects to consider in the design, such us the polarization, index contrast, single mode or multimode operation and propagation

\footnotetext{
${ }^{3}$ The expressions for the upper and lower cladding are the same except for a minus sign in the component $k_{y c l a d d}$, here we show them together
} 

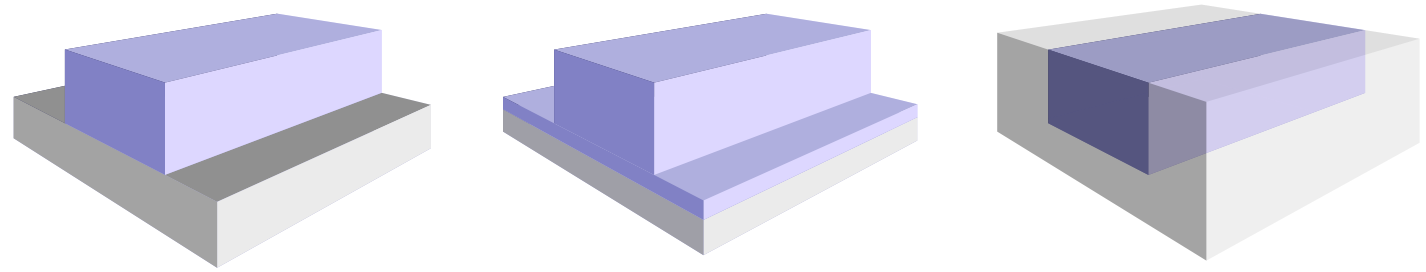

Fig. 2.2 Rectangular waveguides consisting of stripe, rib and buried structures on a planar substrate, from left to right, respectively. The refractive index of the core (blue colored material) is designed to be higher than the one of the cladding (grey colored material).

losses. The higher the index contrast the stronger the confinement of the field, which allows smaller bend radius and thus the densely integration of multiple waveguides in a chip. The most employed material technology is silicon for the core and silica for the cladding, by using SOI wafers. These configuration presents a high-index contrast so that light is easily confined within the waveguide for either TE or TM polarizations.

In the next sections we explore light propagation through non-homogeneous waveguides made of periodic dielectric structures. Based on mathematical formalism, we will study in detail how some of the effects obtained in homogeneous media can be distorted when dealing with periodically patterned waveguides with a smaller pitch than the wavelength of light, thus giving rise to new optical phenomena with interesting properties.

\subsection{Subwavelength gratings}

At the end of the 19th century, Heinrich Hertz conducted a series of experiments to prove Maxwell's predictions on electromagnetic waves [47]. He used a dipole transmitter and a detector surrounded by cylindrical metal mirrors to study the polarization of waves by passing light through a grid of wires, see Fig. 2.3. It was shown that transmission was achieved when the wires were perpendicular to the dipoles, while no signal was collected by the detector when the wires were placed parallel. This effect was later demonstrated to have its origin in the distance between the wires, when it was found that the pitch of the grid must be much smaller than the wavelength of the propagating wave. More detailed theoretical work on periodically layered media was presented by Rayleigh [48] in the following years, showing the birefringence effect that arises from wave propagation through subwavelength structures. More recently, Rytov demonstrated that periodic layered materials behaves like homogeneous media with an effective dielectric permittivity depending on the wave polarization [49]. In the optical domain, subwavelength structures were discovered as an inspiration by the eyes of night-flying 


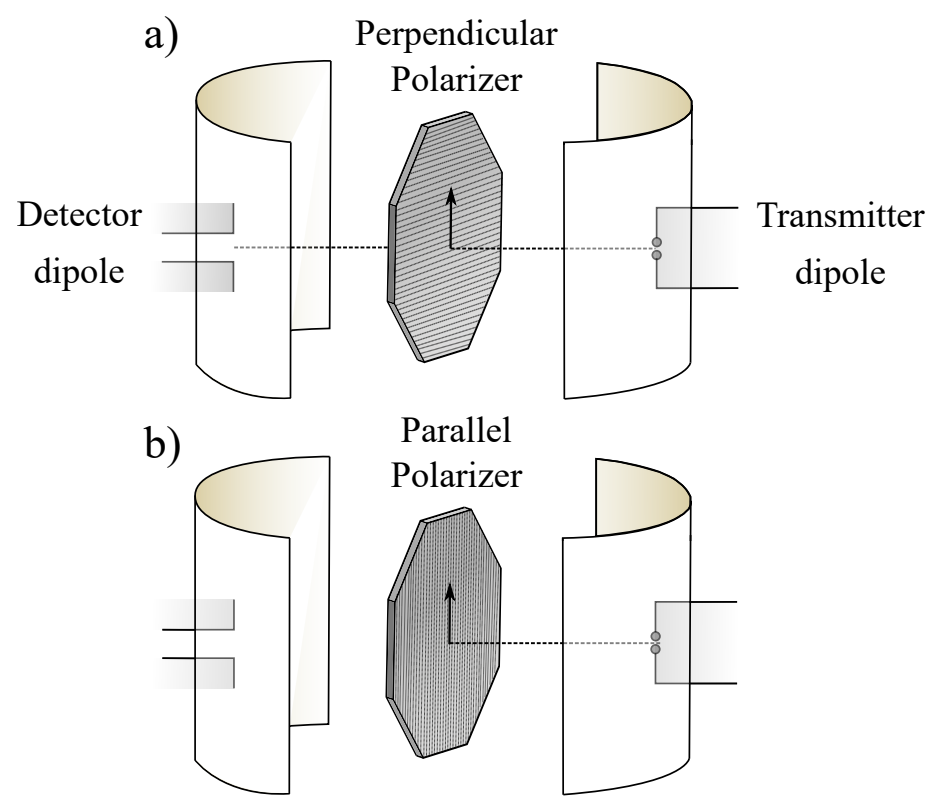

Fig. 2.3 Hertz's experiment on electromagnetic wave propagation through subwavelength wire grid polarizers. a) The electromagnetic wave pass through the polarizing filter when the wires are perpendicular to the dipoles and b) transmission is blocked when they are parallel.

moths and they were used as lenses with reduced reflectance effects [50, 51]. Since then, optical subwavelength devices have emerged for all kind of applications in free-space optics, polarizers and specially in integrated photonics with the growth of the CMOS industry [52-54, 27].

In this section we will focus on the fundamentals of electromagnetic wave propagation trough periodic structures in the subwavelength regime. First, Rytov's demonstration is presented for the homogenization of layered media by using effective medium theory. Then we apply this formalism to optical integrated waveguides based on subwavelength structures for multimode and broadband operation.

\subsubsection{Electromagnetic waves in finely layered media}

Let us consider an infinite layered structure as the one shown in Fig. 2.4 with a period $\Lambda$ consisting of two homogeneous materials with thicknesses $a, b$ and refractive indices $n_{1}, n_{2}$, respectively. Several studies attempted to characterize the electric parameters of this type of stratified medium, as in the case of Bruggeman's work in 1935 [55]. However, it was not until 1956 when Rytov demonstrated that layered structures with very small thicknesses compared to the wavelength, could behave like homogeneous and anisotropic materials [49]. 


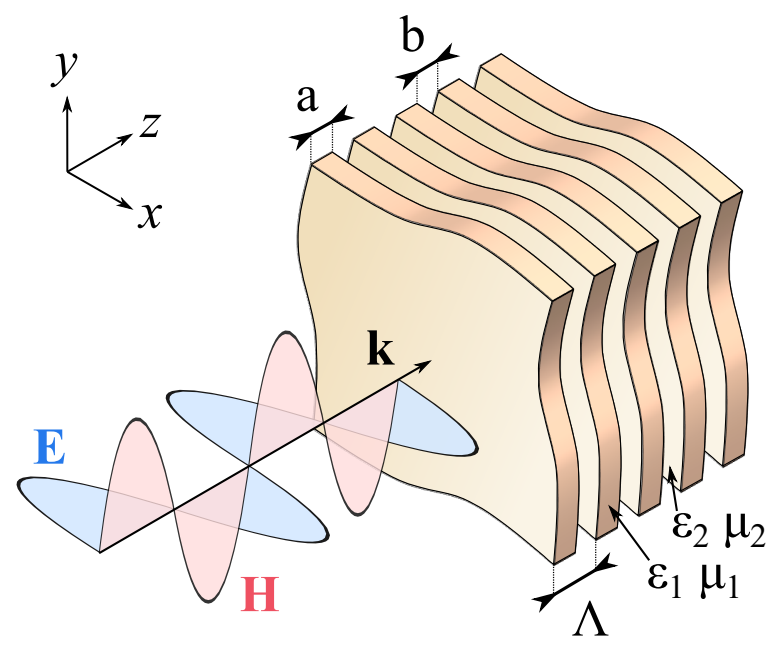

Fig. 2.4 Finely stratified structure consisting of two layers of electric permittivity $\epsilon_{1,2}$ and magnetic permeability $\mu_{1,2}$, respectively, repeated along the $z$ axis with a certain period $\Lambda$.

Let us now apply Maxwell's equations for the calculation of the effective index of the medium by following Rytov's derivation [56]. Considering the geometry of Fig. 2.4, it is clear that there are only three cases of electromagnetic wave propagation through the structure: parallel to the layers (with either the E field or $\mathrm{H}$ field vectors parallel to the interface), and perpendicular to the layers.

\section{Propagation along the layers and E vector parallel to the interface}

Wave propagation in the $x$ direction polarized with $\mathbf{E}$ vector along $y$ axis can be described by harmonic time dependence Maxwell's equations in the interface between two consecutive layers, see Fig. 2.5. Those electromagnetic components that are different from zero are the electric field $\mathbf{E}=\mathbf{E}_{y}$, and the magnetic field $\mathbf{H}_{z}$ and $\mathbf{H}_{x}$. Therefore, in

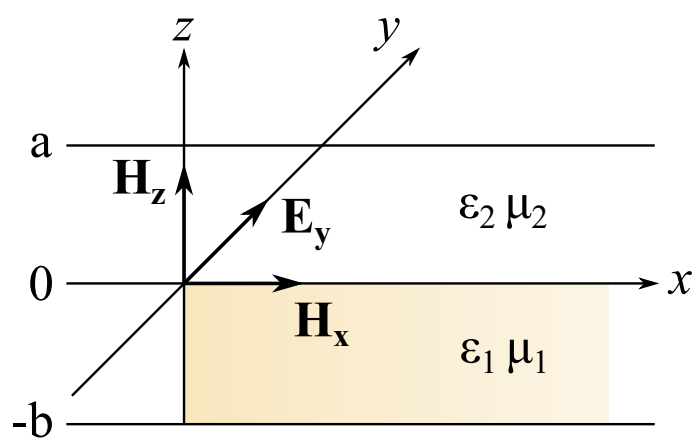

Fig. 2.5 Electromagnetic components distribution for a propagating wave along the layers with $\mathbf{E}$ vector parallel to the interface. 
each layer these fields satisfy the following equations ${ }^{4}$

$$
\begin{aligned}
& \frac{\partial \mathbf{E}}{\partial z}=-i k \mu \mathbf{H}_{x}, \\
& \frac{\partial \mathbf{E}}{\partial x}=i k \mu \mathbf{H}_{z}, \\
& \frac{\partial \mathbf{H}_{z}}{\partial x}-\frac{\partial \mathbf{H}_{x}}{\partial z}=i k \epsilon \mathbf{E}
\end{aligned}
$$

where $k=w / c$ is the wavevector, $\epsilon$ is the electric permittivity and $\mu$ the magnetic permeability of the material, which change periodically along the $z$ axis: $\epsilon_{1,2}$ and $\mu_{1,2}$ for the first and second layer, respectively.

The solutions of Eqs. 2.2.1 for electric and magnetic fields will be in the form

$$
\mathbf{E}=U(z) e^{i k n x}, \mathbf{H}_{x}=V(z) e^{i k n x}, \mathbf{H}_{z}=W(z) e^{i k n x}
$$

where $U, V$ and $W$ are functions of $z$, and $n$ is the refractive index of the medium. Substituting in Eqs. 2.2.1 we obtain

$$
\begin{aligned}
& \frac{\partial U}{\partial z}=-i k \mu V, \\
& n U=\mu W, \\
& \frac{\partial V}{\partial z}-i k n W=-i k \epsilon U .
\end{aligned}
$$

In the interface between two consecutive layers, let us consider $\epsilon_{1}, \mu_{1}$ constants from $z=0$ to $z=a$ and $\epsilon_{2}, \mu_{2}$ for $z=-b$ to $z=0$, see Fig. 2.5. In the first layer, for $0 \leq z \leq a$ exact solutions of Eqs. 2.2.3 are

$$
\begin{aligned}
& U_{1}=A \cos \alpha_{1} z+B \sin \alpha_{1} z, \\
& V_{1}=\frac{\alpha_{1}}{i k \mu_{1}}\left(A \sin \alpha_{1} z-B \cos \alpha_{1} z\right), \\
& W_{1}=\frac{n}{\mu_{1}}\left(A \cos \alpha_{1} z+B \sin \alpha_{1} z\right), \\
& \alpha_{1}=\sqrt{k\left(n_{1}^{2}-n^{2}\right)}, \quad n_{1}^{2}=\epsilon_{1} \mu_{1},
\end{aligned}
$$

\footnotetext{
${ }^{4}$ Note that the time dependency is expressed with a minus sign as $e^{-i w t}$ so that the sign of $i$ is reversed compared to Rytov equations, where it is expressed with a positive sign.
} 
and in the second layer for $-b \leq z \leq 0$

$$
\begin{aligned}
U_{2} & =C \cos \alpha_{2} z+D \sin \alpha_{2} z, \\
V_{2} & =\frac{\alpha_{2}}{i k \mu_{2}}\left(C \sin \alpha_{2} z-D \cos \alpha_{2} z\right), \\
W_{2} & =\frac{n}{\mu_{2}}\left(C \cos \alpha_{2} z+D \sin \alpha_{2} z\right), \\
\alpha_{2} & =\sqrt{k\left(n_{2}^{2}-n^{2}\right)}, \quad n_{2}^{2}=\epsilon_{2} \mu_{2} .
\end{aligned}
$$

Considering continuity and periodicity conditions for $\mathbf{E}$ and $\mathbf{H}_{x}$ fields among other pairs of layers:

$$
\begin{aligned}
& U_{1}(0)=U_{2}(0), \quad U_{1}(a)=U_{2}(-b), \\
& V_{1}(0)=V_{2}(0), \quad V_{1}(a)=V_{2}(-b),
\end{aligned}
$$

we obtain from Eqs. 2.2.4 and 2.2.5 the following set of four homogeneous equations

$$
\begin{aligned}
& C=A, \quad C \cos \alpha_{2} b-D \sin \alpha_{2} b=A \cos \alpha_{1} a+B \sin \alpha_{1} a, \\
& D=p B, \quad C \sin \alpha_{2} b-D \cos \alpha_{2} b=-\xi\left(A \sin \alpha_{1} a-B \cos \alpha_{1} a\right),
\end{aligned}
$$

where

$$
\xi=\frac{\mu_{2} \alpha_{1}}{\mu_{1} \alpha_{2}}
$$

By equating to zero the determinant of the system of Eqs. 2.2.7, we obtain the following dispersion equation, which determines $n$ as a function of $k$ :

$$
\left(1+\xi^{2}\right) \sin \alpha_{1} a \sin \alpha_{2} b+2 \xi\left(1-\cos \alpha_{1} a \cos \alpha_{2} b\right)=0
$$

We can solve Eq. 2.2.9 for $\xi$, thus obtaining the following two equations:

$$
\begin{aligned}
& \tan \left(\frac{\alpha_{2} b}{2}\right) \cot \left(\frac{\alpha_{1} a}{2}\right)=-\xi, \\
& \tan \left(\frac{\alpha_{2} b}{2}\right) \cot \left(\frac{\alpha_{1} a}{2}\right)=\frac{-1}{\xi} .
\end{aligned}
$$

The first of Eqs. 2.2.10 yields a wave in which $\mathbf{E}=\mathbf{E}_{y}$ and $\mathbf{H}_{z}$ are even functions around its middle for $z=0$, while the component $\mathbf{H}_{x}$ is odd so that its mean field over the period $\Lambda$ of the structure is equal to zero. Therefore, the wave is purely transverse with components only in $\mathbf{E}_{y}$ and $\mathbf{H}_{z}$ fields. Similarly, the second of Eqs. 2.2.10 corresponds to a wave in which $\mathbf{H}_{x}$ is even and $\mathbf{E}$ and $\mathbf{H} z$ are odd respect to the center of the layers; thereby 
the mean field has only the longitudinal component $\mathbf{H}_{x}$. However, such a wave will be attenuated for distances of the order of $\Lambda / 2 \pi$ in the $x$ direction, thus this component can be excluded from the analysis. As a result, we denote the mean value of $\mathbf{E}_{y}$ and $\mathbf{H}_{z}$ over the period $\Lambda$ by $\overline{\mathbf{E}}_{y}$ and $\overline{\mathbf{H}}_{z}$. It is necessary now to introduce the effective permeabilities $\epsilon^{e}$ and $\mu^{e}$ by the equations

$$
n=\sqrt{\mu^{e} \epsilon^{e}}, \quad \frac{\overline{\mathbf{E}}_{y}}{\overline{\mathbf{H}}_{z}}=\sqrt{\frac{\mu^{e}}{\epsilon^{e}}},
$$

where $n$ is the root of the first of Eqs 2.2.10 and

$$
\overline{\mathbf{E}}_{y}=\frac{1}{\Lambda}\left(\int_{0}^{a} U_{1} d z+\int_{-b}^{0} 2 U_{2} d z\right)
$$

Substituting $U_{1}$ and $U_{2}$ from Eqs. 2.2.4 and 2.2.5 and using relations 2.2.7 to calculate $\overline{\mathbf{E}}_{y}$, and similarly $\overline{\mathbf{H}}_{z}$, we get

$$
\frac{\overline{\mathbf{E}}_{y}}{\overline{\mathbf{H}}_{z}}=\frac{\mu_{1} \alpha_{2}^{2}-\mu_{2} \alpha_{1}^{2}}{n\left(\alpha_{2}^{2}-\alpha_{1}^{2}\right)} .
$$

Equating this with the second expression of Eqs. 2.2.11 and solving the resulting equations for $\epsilon^{e}$ and $\mu^{e}$, we obtain

$$
\begin{aligned}
\epsilon^{e} & =\frac{n^{2}\left(\alpha_{2}^{2}-\alpha_{1}^{2}\right)}{\mu_{1} \alpha_{2}^{2}-\mu_{2} \alpha_{1}^{2}}, \\
\mu^{e} & =\frac{\mu_{1} \alpha_{2}^{2}-\mu_{2} \alpha_{1}^{2}}{\alpha_{2}^{2}-\alpha_{1}^{2}} .
\end{aligned}
$$

Considering small thicknesses $a$ and $b$, so that $\tan \left(\alpha_{2} b / 2\right) \approx \alpha_{2} b / 2$ and $\cot \left(\alpha_{1} a / 2\right) \approx$ $\left(\alpha_{1} a / 2\right)^{-1}$ we find from Eqs. 2.2.14 that

$$
\epsilon^{e}=\bar{\epsilon}, \quad \mu^{e}=\tilde{\mu}
$$

where

$$
\begin{aligned}
& \bar{\epsilon}=\frac{1}{\Lambda}\left(a \epsilon_{1}+b \epsilon_{2}\right), \\
& \frac{1}{\widetilde{\mu}}=\frac{1}{\Lambda}\left(\frac{a}{\mu_{1}}+\frac{b}{\mu_{2}}\right),
\end{aligned}
$$

for the average value of $\epsilon$ and $1 / \mu$ over the period $\Lambda^{5}$.

\footnotetext{
${ }^{5}$ A more exact estimation considering the cubic terms in the expansion for the correction of the effective values $\epsilon^{e}$ and $\mu^{e}$ is detailed in [49].
} 
Propagation along the layers and $\mathrm{H}$ vector parallel to the interface

In this case, the components different from zero are $\mathbf{E}_{x}, \mathbf{E}_{z}$ and $\mathbf{H}_{y}=\mathbf{H}$, see Fig. 2.6, yielding the following Maxwell's equations

$$
\begin{aligned}
& \frac{\partial \mathbf{H}}{\partial z}=i k \epsilon \mathbf{E}_{x}, \\
& \frac{\partial \mathbf{H}}{\partial x}=-i k \epsilon \mathbf{E}_{z}, \\
& \frac{\partial \mathbf{E}_{x}}{\partial z}-\frac{\partial \mathbf{E}_{z}}{\partial x}=i k \mu \mathbf{H} .
\end{aligned}
$$

Considering continuity and periodicity conditions, all formulas derivation in this case can be obtained from the previous one by replacing $\mathbf{H}, \mathbf{E}, \mu, \epsilon$ by $\mathbf{E}, \mathbf{H}, \epsilon, \mu$, respectively. Therefore, the main field over the period is a pure transverse wave with components $\overline{\mathbf{H}}_{y}$ and $\overline{\mathbf{E}}_{z}$ that satisfy Maxwell's equations:

$$
\begin{aligned}
& \frac{\partial \overline{\mathbf{H}}_{y}}{\partial x}=-i k \epsilon^{e} \overline{\mathbf{E}}_{z}, \\
& \frac{\partial \overline{\mathbf{E}}_{z}}{\partial x}=-i k \mu^{e} \overline{\mathbf{H}}_{y},
\end{aligned}
$$

where the effective values of $\epsilon^{e}$ and $\mu^{e}$ are expressed as

$$
\begin{aligned}
\epsilon^{e} & =\frac{\epsilon_{1} \alpha_{2}^{2}-\epsilon_{2} \alpha_{1}^{2}}{\alpha_{2}^{2}-\alpha_{1}^{2}}, \\
\mu^{e} & =\frac{n^{2}\left(\alpha_{2}^{2}-\alpha_{1}^{2}\right)}{\epsilon_{1} \alpha_{2}^{2}-\epsilon_{2} \alpha_{1}^{2}},
\end{aligned}
$$

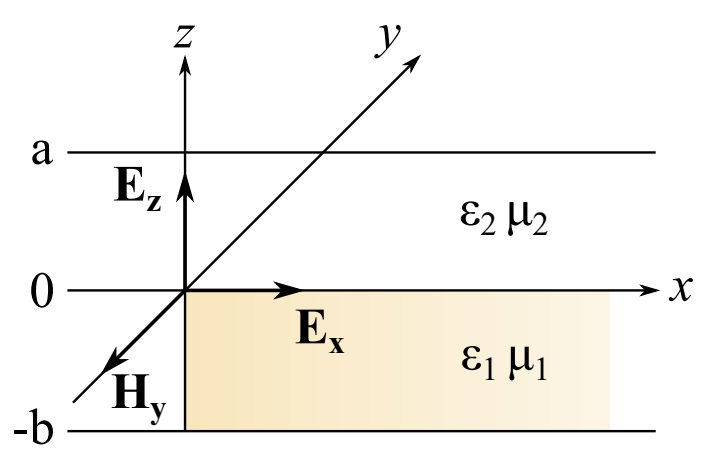

Fig. 2.6 Electromagnetic components distribution for a propagating wave along the layers with $\mathbf{H}$ vector parallel to the interface. 
obtained from Eqs. 2.2.14 by the substitution previously shown. The equation for $n$ is in this case is

$$
\frac{\alpha_{2}}{\epsilon_{2}} \tan \left(\frac{\alpha_{2} b}{2}\right)=-\frac{\alpha_{1}}{\epsilon_{1}} \tan \left(\frac{\alpha_{1} a}{2}\right)
$$

that for small values of $a$ and $b$ we obtain

$$
\epsilon^{e}=\widetilde{\epsilon}, \quad \mu^{e}=\bar{\mu}
$$

where

$$
\begin{aligned}
& \frac{1}{\widetilde{\epsilon}}=\frac{1}{\Lambda}\left(\frac{a}{\epsilon_{1}}+\frac{b}{\epsilon_{2}}\right), \\
& \bar{\mu}=\frac{1}{\Lambda}\left(a \mu_{1}+b \mu_{2}\right) .
\end{aligned}
$$

\section{Propagation perpendicular to the interface}

In the last case, the wave propagation occurs along the $z$ axis, normal to the interface, see Fig. 2.7. Those components different from zero are $\mathbf{E}_{x}=\mathbf{E}$ and $\mathbf{H}_{y}=\mathbf{H}$ and satisfy the equations

$$
\begin{aligned}
& \frac{\partial \mathbf{E}}{\partial z}=i k \mu \mathbf{H}, \\
& \frac{\partial \mathbf{H}}{\partial z}=i k \epsilon \mathbf{E} .
\end{aligned}
$$

According to the Floquet's theorem [57], now the solutions to Eqs. 2.2.23 will be in the form

$$
\mathbf{E}=U(z) e^{i k n z}, \mathbf{H}=V(z) e^{i k n z},
$$

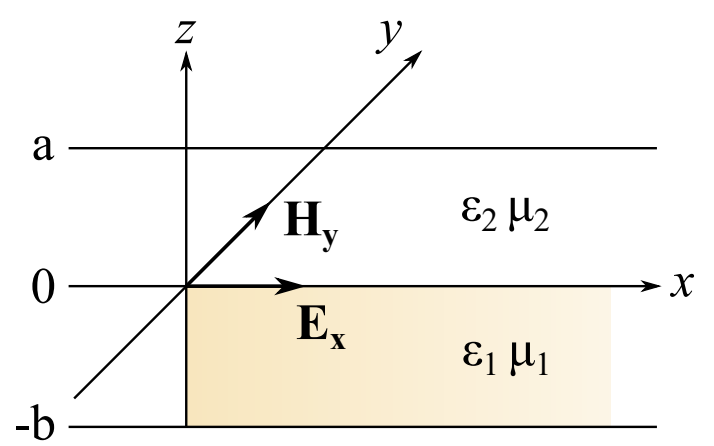

Fig. 2.7 Electromagnetic components distribution for a propagating wave normal to the layers. 
where $U$ and $V$ are periodic functions of $z$ with a certain period $\Lambda$.

Substituting in Eqs. 2.2.23 and solving for each separate layer, we obtain for $0 \leq z \leq a$

$$
\begin{aligned}
& U_{1}=e^{-i k n z}\left(A e^{-i \alpha_{1} z}+B e^{i \alpha_{1} z}\right), \\
& V_{1}=-\frac{\alpha_{1}}{k \mu_{1}} e^{-i k n z}\left(A e^{-i \alpha_{1} z}+B e^{i \alpha_{1} z}\right), \\
& \alpha_{1}=k n_{1}=k \sqrt{\epsilon_{1} \mu_{1}}
\end{aligned}
$$

and in the second layer for $-b \leq z \leq 0$

$$
\begin{aligned}
& U_{2}=e^{-i k n z}\left(C e^{-i \alpha_{2} z}+D e^{i \alpha_{2} z}\right), \\
& V_{2}=-\frac{\alpha_{2}}{k \mu_{2}} e^{-i k n z}\left(C e^{-i \alpha_{2} z}+D e^{i \alpha_{2} z}\right), \\
& \alpha_{2}=k n_{2}=k \sqrt{\epsilon_{2} \mu_{2}} .
\end{aligned}
$$

Considering continuity and periodicity conditions expressed in Eqs. 2.2.6 on $U$ and $V$, we get the following set of four equations

$$
\begin{aligned}
& C+D=A+B, \quad e^{i k n b}\left(C e^{i \alpha_{2} b}+D e^{-i \alpha_{2} b}\right)=e^{-i k n a}\left(A e^{-i \alpha_{1} a}+B e^{i \alpha_{1} a}\right), \\
& C-D=\xi(A-B), \quad e^{i k n b}\left(C e^{i \alpha_{2} b}-D e^{-i \alpha_{2} b}\right)=\xi e^{-i k n a}\left(A e^{-i \alpha_{1} a}+B e^{i \alpha_{1} a}\right),
\end{aligned}
$$

where

$$
\xi=\frac{\mu_{2} \alpha_{1}}{\mu_{1} \alpha_{2}} \sqrt{\frac{\epsilon_{1} \mu_{2}}{\epsilon_{2} \mu_{1}}} .
$$

Solving the determinant of the system in Eqs. 2.2.25 and 2.2.26 to zero, we get the dispersion equation of $n$ :

$$
\cos k n \Lambda=\cos \alpha_{1} a \cos \alpha_{2} b-\frac{1+\xi^{2}}{2 \xi} \sin \alpha_{1} a \sin \alpha_{2} b .
$$

We now calculate the mean fields $\overline{\mathbf{E}}_{x}=\overline{\mathbf{E}}$ and $\overline{\mathbf{H}}_{y}=\overline{\mathbf{H}}$ over the period $\Lambda$, that satisfy Maxwell's equations

$$
\begin{gathered}
\frac{\partial \overline{\mathbf{E}}_{x}}{\partial z}=i k \mu^{e} \overline{\mathbf{H}}_{y}, \\
\frac{\partial \overline{\mathbf{H}}_{y}}{\partial z}=i k \epsilon^{e} \overline{\mathbf{E}}_{x},
\end{gathered}
$$


for the conditions

$$
n=\sqrt{\epsilon^{e} \mu^{e}}, \quad \frac{\overline{\mathbf{E}}_{x}}{\overline{\mathbf{H}}_{y}}=\frac{\bar{U}}{\bar{V}}=\sqrt{\frac{\mu^{e}}{\epsilon^{e}}},
$$

whence

$$
\epsilon^{e}=\frac{n \overline{\mathbf{H}}_{y}}{\overline{\mathbf{E}}_{x}}, \quad \mu^{e}=\frac{n \overline{\mathbf{E}}_{x}}{\overline{\mathbf{H}}_{y}} .
$$

Solving these equations, we get the expressions for the effective values of $\epsilon^{e}$ and $\mu^{e}$

$$
\begin{aligned}
& \epsilon^{e}=\bar{\epsilon}\left[1+\frac{i k a b\left(\mu_{1} \epsilon_{2}-\mu_{2} \epsilon_{1}\right)}{4 \Lambda \sqrt{\overline{\epsilon \mu}}}\right], \\
& \mu^{e}=\bar{\mu}\left[1-\frac{i k a b\left(\mu_{1} \epsilon_{2}-\mu_{2} \epsilon_{1}\right)}{4 \Lambda \sqrt{\overline{\epsilon \mu}}}\right] .
\end{aligned}
$$

Considering small thicknesses for $a$ and $b$ the correction terms of Eqs. 2.2.33 can be neglected.

Joining together all the cases previously considered, we get the set of Maxwell's equations for the mean fields

$$
\begin{array}{lll}
\frac{\partial \overline{\mathbf{E}}_{y}}{\partial x}=i k \widetilde{\mu} \overline{\mathbf{H}}_{z}, & \frac{\partial \overline{\mathbf{E}}_{x}}{\partial z}=i k \bar{\mu} \overline{\mathbf{H}}_{y}, & \frac{\partial \overline{\mathbf{E}}_{z}}{\partial x}=-i k \bar{\mu} \overline{\mathbf{H}}_{y} \\
\frac{\partial \overline{\mathbf{H}}_{y}}{\partial x}=-i k \widetilde{\epsilon} \overline{\mathbf{E}}_{z}, & \frac{\partial \overline{\mathbf{H}}_{z}}{\partial x}=i k \bar{\epsilon} \overline{\mathbf{E}}_{y}, & \frac{\partial \overline{\mathbf{H}}_{y}}{\partial z}=i k \bar{\epsilon} \overline{\mathbf{E}}_{x} .
\end{array}
$$

This set of Maxwell's equations characterize a medium whose permittivity and permeability are governed by singly degenerated tensors with the following principal axes and values:

$$
\begin{aligned}
& \epsilon_{1}^{e}=\epsilon_{2}^{e}=\bar{\epsilon}=\frac{\epsilon_{1} a+\epsilon_{2} b}{\Lambda}, \\
& \epsilon_{3}^{e}=\widetilde{\epsilon}=\frac{\epsilon_{1} \epsilon_{2} \Lambda}{\epsilon_{2} a+\epsilon_{1} b}, \\
& \mu_{1}^{e}=\mu_{2}^{e}=\bar{\mu}=\frac{\mu_{1} a+\mu_{2} b}{\Lambda}, \\
& \mu_{3}^{e}=\widetilde{\mu}=\frac{\mu_{1} \mu_{2} \Lambda}{\mu_{2} a+\mu_{1} b} .
\end{aligned}
$$

Therefore, the resulting layered material behaves like a homogeneous and anisotropic medium with properties of an uniaxial crystal, and characterized by the dielectric and magnetic permittivities $\epsilon^{e}$ and $\mu^{e}$. 


\subsubsection{Waveguiding in subwavelength structures}

Let us now consider a certain dielectric structure of refractive index $n_{1}$ with a thickness $a$, width $w$ and height $h$ repeated along the $z$ axis with a periodicity $\Lambda$, see Fig. 2.8. The gap in between the periodic elements is filled with a medium of refractive index $n_{2}$ and the whole structure is placed over a substrate of refractive index $n_{3}$. As it has been shown in the previous section, wave propagation through the structure is possible for small periodicities compared to the wavelength. Such a periodic structure can thereby be modeled as a homogeneous anisotropic material with their corresponding electric permittivities and magnetic permeabilities, following Rytov's derivation. Considering dielectric materials with a permeability value of $\mu^{e}=1$, we can calculate the effective index as

$$
n^{e}=\sqrt{\epsilon^{e}}
$$

Substituting in Eqs. 2.2.35, we obtain

$$
\begin{aligned}
& n_{\|}^{2}=\frac{a}{\Lambda} n_{1}^{2}+\left(1-\frac{a}{\Lambda}\right) n_{2}^{2}, \\
& \frac{1}{n_{\perp}^{2}}=\frac{a}{\Lambda} \frac{1}{n_{1}^{2}}+\left(1-\frac{a}{\Lambda}\right) \frac{1}{n_{2}^{2}},
\end{aligned}
$$

where $n_{\|}$and $n_{\perp}$ is the effective index of the effective medium for a TE polarization wave with the $\mathbf{E}$ vector parallel and perpendicular to the structure periodicity, $k_{1}$ and $k_{2}$ in Fig. 2.8 , respectively.

This formalism is valid for those structures with a small enough pitch to avoid diffraction, entering in the so-called subwavelength regime [26]. Many applications are based on waveguides as the one shown in Fig. 2.8 using the SOI platform, where the core

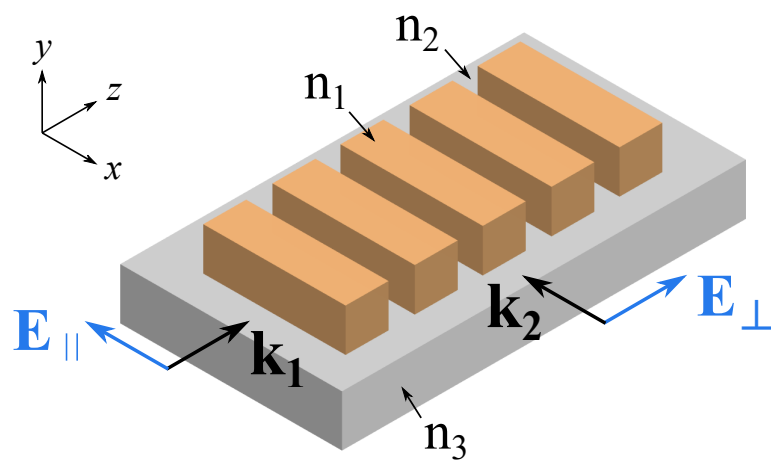

Fig. 2.8 Schematic of a periodic dielectric waveguide based on an array of subwavelength elements on a substrate. 
is made of silicon ( $\left.n_{1} \sim 3.5\right)$, upper cladding of silica or air ( $\left.n_{2} \sim 1-1.45\right)$ and a substrate of silica $\left(n_{3} \sim 1.45\right)$. Light propagation through these segmented waveguides is modeled by the Bloch-Floquet modes [58, 59], similar to an electron in a natural crystal. The propagation of a Bloch mode in a periodic waveguide is defined as ${ }^{6}$

$$
\mathbf{E}(x, z+\Lambda)=\mathbf{E}_{B},(x, z) e^{-\gamma \Lambda}
$$

where $\mathbf{E}_{B},(x, z)$ is the field distribution of the Bloch mode over a period $\Lambda$ and $\gamma_{B}$ is its complex propagation constant, expressed as

$$
\gamma_{B}=\alpha_{B}+i k_{B}=\alpha_{B}+n_{B} i \frac{2 \pi}{\Lambda},
$$

where $\alpha_{B}$ and $k_{B}$ are the attenuation constant and the wavevector, respectively, and $n_{B}$ the effective index of the Bloch mode. Therefore, for a given period $\Lambda$ the behavior of light propagated along SWG waveguides will be strongly dependent on the operating frequency. Figure 2.9 shows the dispersion relation of a certain periodic structure as the angular frequency $w$ versus the wavector of the Bloch mode $k$. In the subwavelength regime, for low frequencies, light behaves like in a homogeneous waveguide and reflection effects in the interfaces are suppressed, see field distribution of Fig. 2.9. As we increase the frequency, the wavelength is comparable to the structure periodicity so that light is reflected backwards. In this case, the periodic waveguide behaves like a Bragg mirror and the propagating mode is completely attenuated after a certain distance. Above this region for even higher frequencies, part of the light is radiated into the free space and high-order bands appear in the band diagram of the resulting photonic crystal.

To determine whether we are working in the subwavelength regime, a first approximation considering the relationship between the periodicity of the structure and the effective index of the Bloch mode is usually expressed as follows

$$
n_{B}<\frac{\lambda}{2 \Lambda}
$$

where $\lambda$ is the operating wavelength, assuring that we work below the Bragg threshold. Within the subwavelength region, waveguiding effects must be considered when dealing with structures as in the case of Fig. 2.8, since the waveguide does not extend infinitely in $x$ and $y$ directions. It should be also noted that as we get closer to the Bragg regime, more accurate analysis must be carried out by using numerical methods as the expressions of Eqs. 2.2.37 are only valid in the deep subwavelength region.

\footnotetext{
${ }^{6}$ A more exhaustive study of Bloch-Floquet formalism is detailed in $[58,59]$, here we just provide the basis for the understanding of general concepts.
} 


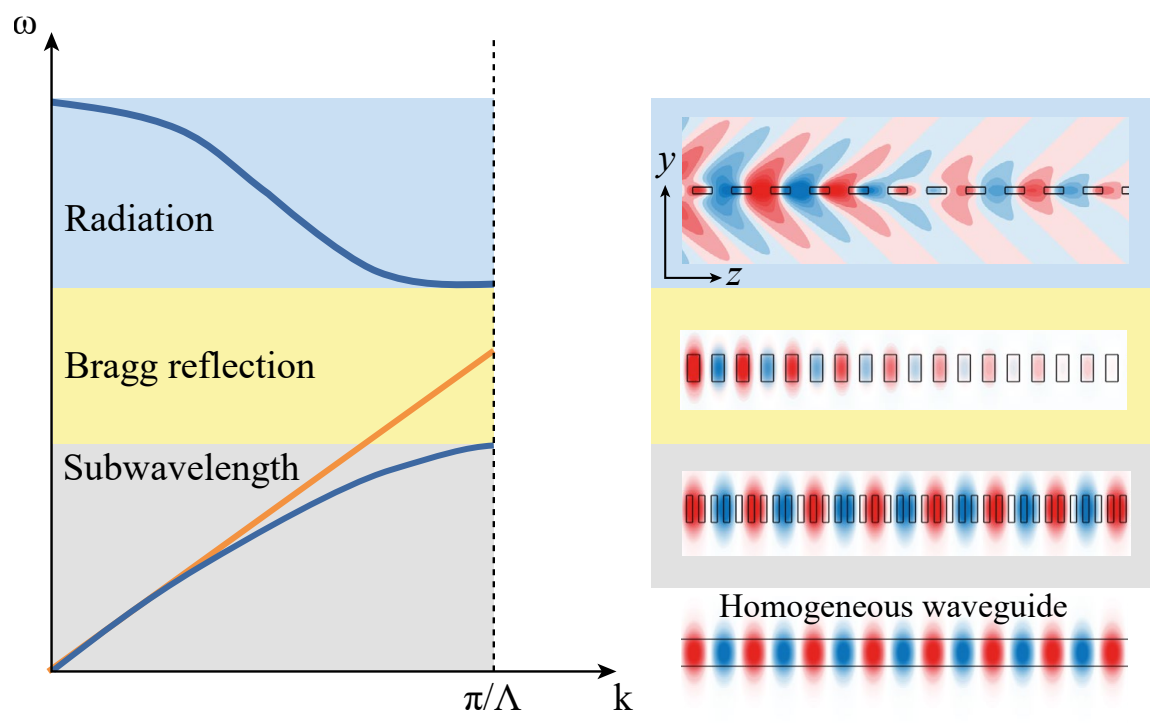

Fig. 2.9 Light behaviour in a periodic dielectric waveguide [27]: schematic dispersion diagram and field distribution of its corresponding region, left and right part, respectively.

\subsubsection{Multimode operation}

As in conventional rectangular waveguides, SWG structures can support several modes propagated along the periodic interface. In Fig. 2.10 we consider a multimode waveguide with a width $w$ made of a SWG elements with refractive indices $n_{\|}$and $n_{\perp}$, see Eqs. 2.2.37. Optical modes will be confined within the waveguide as the form

$$
\varphi_{m}(x) \approx \sin \left(k_{x, m} x\right)
$$

where the wavevector in the $x$ axis is defined by

$$
k_{x, m} \approx m \pi / w,
$$

for the $m$ th order guided mode. In turn, the longitudinal component in the $z$ axis depends on the effective index of the modes as

$$
n_{m}^{e}=\frac{k_{z, m} \lambda}{2 \pi},
$$

which is used to design the beat length of certain devices based on multimode operation.

Considering the elliptical dispersion relation of the uniaxial resulting crystal

$$
\left(\frac{k_{z, m}}{n_{\|}}\right)^{2}+\left(\frac{k_{x, m}}{n_{\perp}}\right)^{2}=\left(\frac{2 \pi}{\lambda}\right)^{2},
$$




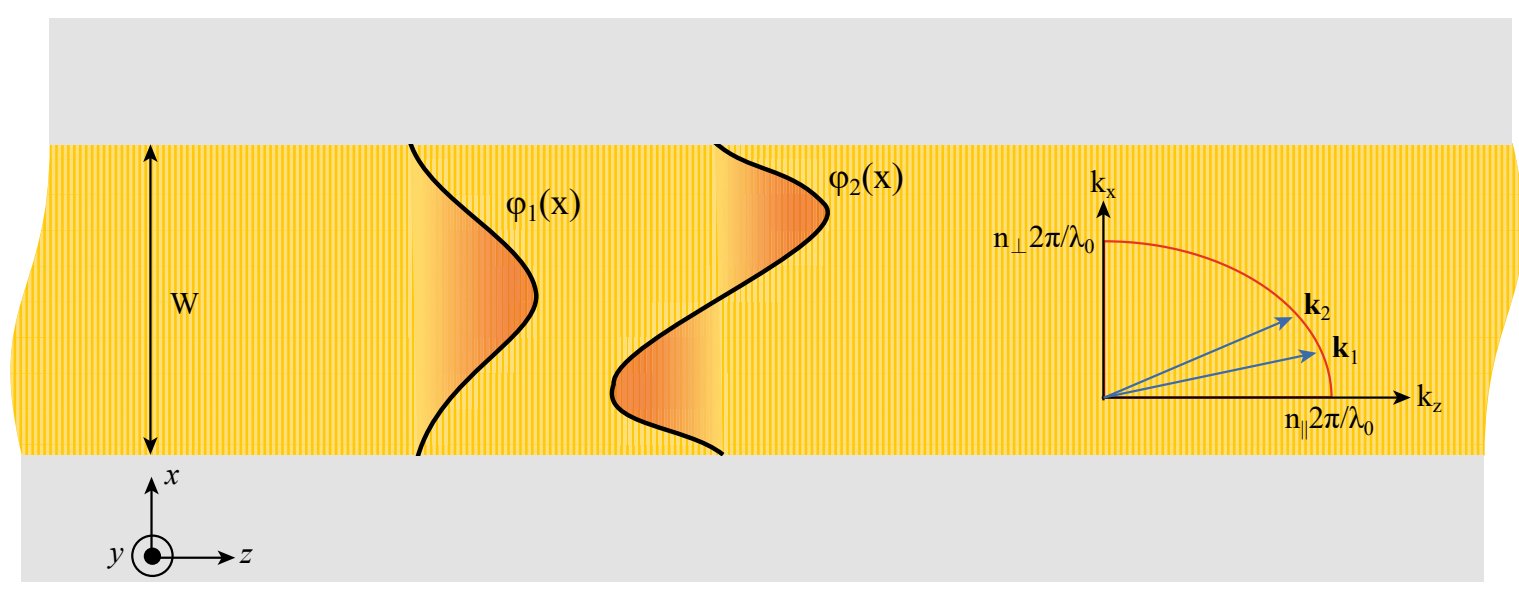

Fig. 2.10 Top view of a multimode subwavelength waveguide sketch of a certain width $w$ with the representation of the two first lower order modes [27].

and for the paraxial approximation $k_{x}<<2 \pi / \lambda$, we can express the modes effective indices as follows

$$
n_{m}^{e} \approx n_{\|}-\frac{n^{2} \lambda^{2} n_{\|}^{2}}{8 w^{2} n_{\perp}^{2}} .
$$

We can now define the beat length $L_{\pi}$ between the lowest order modes by the following expression

$$
L_{\pi}=\frac{2 \pi}{k_{z, 1}-k_{z, 2}} \approx \frac{4 w^{2} n_{\perp}^{2}}{3 \lambda n_{\|}} .
$$

By properly designing the SWG structure we can engineer the effective indices $n_{\|}$and $n_{\perp}$ to provide an active control on the beat length, thus becoming wavelength-independent and therefore enable broadband operation. This fact allow us to develop novel devices based on SWG structures with improved properties compared to conventional homogeneous waveguides. Specifically, in the chapter 3 we further investigate multimode operation in SWGs for high-performance sensing based on the dispersion effects near the Bragg regime.

\subsection{Photonic crystals}

In the previous section, we have seen how periodic structures behave like homogeneous waveguides when the pitch is much smaller than the effective wavelength of the propagating light. It has also been shown that for larger periodicities light is reflected and radiated and thus the waveguide becomes lossy. This fact, however, is not entirely 

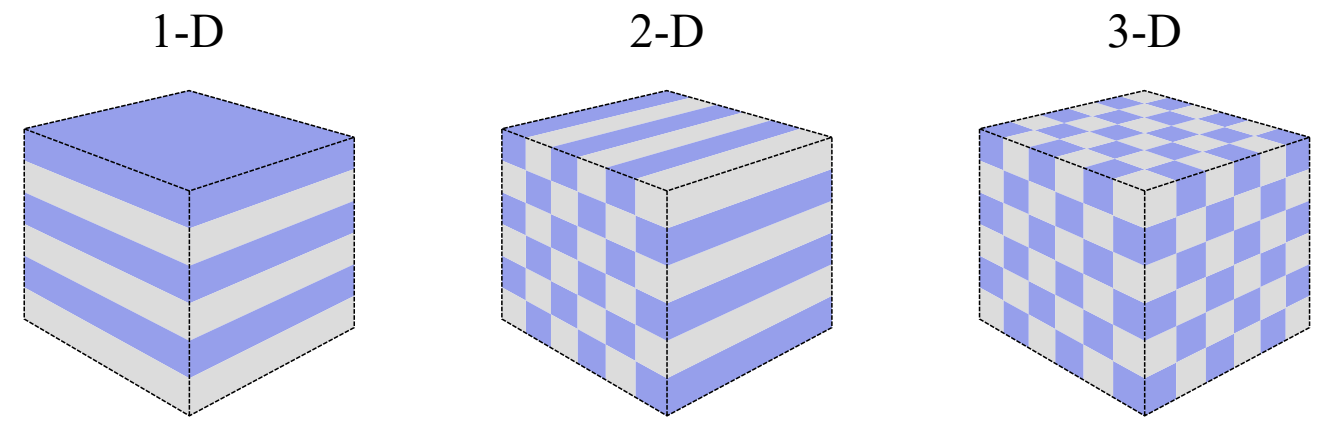

Fig. 2.11 Sketch of periodic structures as one-dimensional (1D), two-dimensional (2D) and three-dimensional (3D) photonic crystals. The colors represent different materials with their respective dielectric constants.

accurate and loss-less transmission can be accomplished beyond the subwavelength regime. In solid-state physics, electrons avoid scattering effects from the periodic potential of a crystal lattice because they propagate as waves that meet certain criteria so that transmission without scattering can be achieved.

In optics, the analogue is the photonic crystal, consisting of macroscopic structures with a given periodic refractive index instead of atoms with periodic potentials. Photonic crystals can extend in one, two or three spatial directions, see Fig. 2.11, and avoid refractions and reflections of light even when the refractive index contrast of the materials is large, thus obtaining a loss-less periodic dielectric medium. These structures can manipulate the propagation direction of light and prevent it in some specific directions and for certain frequencies, the so-called photonic bandgaps. In addition, they allow propagation with some anomalous effects such as the slow light phenomena, where light is drastically slowed down which in turn can be used in several useful ways. In this chapter, we provide a short introduction to light behavior in photonic crystals, and discuss the main optical properties obtained from the mathematical derivation. Then we focus on one-dimensional structures and provide the basis to understand the band structure formation in periodic dielectric structures similar to the ones presented in the following chapters.

\subsubsection{Eigenvalue problem of electromagnetic waves}

Light behavior in a photonic crystal is governed by Maxwell's equations, as we have seen in the previous sections. However, here the equations are proposed as a linear Hermitian eigenvalue problem, similar to what occurs in quantum mechanics with the Schrödinger equation [60]. Let us detail again the macroscopic Maxwell's equations which are usually 
formulated as

$$
\begin{aligned}
& \nabla \cdot \mathbf{D}=\rho, \\
& \nabla \cdot \mathbf{B}=0, \\
& \nabla \times \mathbf{E}+\frac{\partial \mathbf{B}}{\partial t}=0, \\
& \nabla \times \mathbf{H}-\frac{\partial \mathbf{D}}{\partial t}=\mathbf{J},
\end{aligned}
$$

where $\mathbf{E}$ and $\mathbf{H}$ are the electric and magnetic fields, $\mathbf{D}$ and $\mathbf{B}$ the electric displacement and magnetic induction, and $\rho$ and $\mathbf{J}$ are the electric charge and current density, respectively.

We consider a periodic dielectric medium consisting of homogeneous materials with a certain permittivity constant as a function of the position vector $\mathbf{r}$, with no free charges and no currents: $\rho=0$ and $\mathbf{J}=0$. The constitutive relations relate the electric and magnetic fields $\mathbf{E}$ and $\mathbf{H}$ to the displacement and magnetic induction $\mathbf{D}$ and $\mathbf{B}$. The components of the electric field and displacement are related for each component via power series in the following form ${ }^{7}$

$$
D_{i} / \epsilon_{0}=\sum_{j} \epsilon_{i j} E_{j}+\sum_{j, k} \chi_{i j k} E_{j} E_{k}+O\left(E^{3}\right)
$$

where $\epsilon_{0} \approx 8.854 \times 10^{-12} \mathrm{Farad} / \mathrm{m}$ is the value of vacuum permittivity. We now consider that we are working in the linear regime, so that $\chi_{i j k}$ terms can be neglected. We also assume macroscopic and isotropic materials, so that $\mathbf{E}(\mathbf{r}, \omega)$ and $\mathbf{D}(\mathbf{r}, \omega)$ relate by the scalar relative permittivity $\epsilon(\mathbf{r}, \omega)$. We ignore dispersion effects of $\epsilon$ and consider a loss-less material, which means that its value is purely real and positive ${ }^{8}$.

Taking into account all these considerations and that the value of $\mu(\mathbf{r})$ can be approximated to unity, we obtain the following constitutive relations

$$
\begin{aligned}
& \mathbf{D}(\mathbf{r})=\epsilon_{0} \epsilon(\mathbf{r}) \mathbf{E}(\mathbf{r}), \\
& \mathbf{B}(\mathbf{r})=\mu_{0} \mathbf{B}(\mathbf{r}),
\end{aligned}
$$

\footnotetext{
7 This mathematical expression is taken from Bloembergen's work in 1965 on nonlinear optics [61].

${ }^{8}$ Negative dielectric constants are interesting descriptions for some materials such as metals, which may be used to develop mixed dielectric-metals photonic crystals $[62,63]$
} 
With all these assumptions, Eqs. 2.3.1 become

$$
\begin{aligned}
& \nabla \cdot[\epsilon(\mathbf{r}) \mathbf{E}(\mathbf{r}, t)]=0, \\
& \nabla \cdot \mathbf{H}(\mathbf{r}, t)=0, \\
& \nabla \times \mathbf{E}(\mathbf{r}, t)+\mu_{0} \frac{\partial \mathbf{H}(\mathbf{r}, t)}{\partial t}=0, \\
& \nabla \times \mathbf{H}(\mathbf{r}, t)-\epsilon_{0} \epsilon(\mathbf{r}) \frac{\partial \mathbf{E}(\mathbf{r}, t)}{\partial t}=0 .
\end{aligned}
$$

The time dependency of both $\mathbf{E}$ and $\mathbf{H}$ can be expressed by expanding the fields into a set of harmonic modes that evolve sinusoidally with time. Therefore, these harmonic modes can be written as a certain pattern multiply by a complex exponential as follows

$$
\begin{aligned}
& \mathbf{H}(\mathbf{r}, t)=\mathbf{H}(\mathbf{r}) e^{-i \omega t}, \\
& \mathbf{E}(\mathbf{r}, t)=\mathbf{E}(\mathbf{r}) e^{-i \omega t} .
\end{aligned}
$$

For a certain frequency, to obtain the field profile of the modes we must insert these expressions in Eqs. 2.3.4. The two divergence equations yield the next condition

$$
\nabla \cdot \mathbf{H}(\mathbf{r})=\nabla \cdot[\epsilon(\mathbf{r}) \mathbf{E}(\mathbf{r})]=0,
$$

which indicates that there are no sources or sinks of electric displacement and magnetic field in the medium. In addition, these fields consist of purely transverse waves perpendicular to the propagation direction, so that for a given plane wave $\mathbf{H}(\mathbf{r})=a e^{i \mathbf{k r}}$ for a wavevector $\mathbf{k}$ Eq. 2.3.6 requires that $\mathbf{a} \cdot \mathbf{k}=0$. Likewise, we now focus on the curl equations

$$
\begin{aligned}
& \nabla \times \mathbf{E}(\mathbf{r})-i \omega \mu_{0} \mathbf{H}(\mathbf{r})=0, \\
& \nabla \times \mathbf{H}(\mathbf{r})+i \omega \epsilon_{0} \epsilon(\mathbf{r}) \mathbf{E}(\mathbf{r})=0 .
\end{aligned}
$$

We now divide the bottom of Eq. 2.3.7 by $\epsilon(\mathbf{r})$ and then take the curl. Then, by using the first equation to eliminate $\mathbf{E}(\mathbf{r})$ and combining $\epsilon_{0}$ and $\mu_{0}$ with the vacuum speed of light we obtain the following expression for the magnetic field $\mathbf{H}(\mathbf{r})$

$$
\nabla \times\left(\frac{1}{\epsilon(r)} \nabla \times \mathbf{H}(\mathbf{r})\right)=\left(\frac{\omega}{c}\right)^{2} \mathbf{H}(\mathbf{r}) .
$$

Eq. 2.3.8 is the master equation and relates the magnetic modes $\mathbf{H}(r)$ with its corresponding frequencies for a given dielectric structure $\epsilon(\mathbf{r})$. Then, we can use the 
second equation of 2.3.7 to obtain the electric field as

$$
\mathbf{E}(\mathbf{r})=\frac{i}{\omega \epsilon_{0} \epsilon(\mathbf{r})} \nabla \times \mathbf{H}(\mathbf{r}) .
$$

This process satisfies the transversality condition $\nabla \cdot \epsilon \mathbf{E}=0$ given that the divergence of a curl is always zero. Similarly, we can also obtain $\mathbf{H}$ from $\mathbf{E}$ in Eq. 2.3.7 as

$$
\mathbf{H}(\mathbf{r})=-\frac{i}{\omega \mu_{0}} \nabla \times \mathbf{E}(\mathbf{r})
$$

For mathematical convenience it is easier to solve the problem in terms of the magnetic field $\mathbf{H}(\mathbf{r})$ rather than the electric field $\mathbf{E}(\mathbf{r})$. The master Eq. 2.3.8 is an eigenvalue problem in which the eigenfunction or eigenvector ${ }^{9}$ is the function itself multiplied by some constant, also called eigenvalue. Specifically, we can express the left side of the master equation with an operator $\hat{\Theta}$ acting on $\mathbf{H}(\mathbf{r})$ as

$$
\hat{\Theta} \mathbf{H}(\mathbf{r})=\left(\frac{\omega}{c}\right)^{2} \mathbf{H}(\mathbf{r})
$$

whence $\hat{\Theta}$ is the differential operation that takes the curl, divides by $\epsilon(\mathbf{r})$ and takes the curl again:

$$
\hat{\Theta} \mathbf{H}(\mathbf{r}) \triangleq \nabla \times\left(\frac{1}{\epsilon(\mathbf{r})} \nabla \times \mathbf{H}(\mathbf{r})\right)
$$

where $\mathbf{H}(\mathbf{r})$ is the eigenvector and $(w / c)^{2}$ the eigenvalues of the system. It should be also noted that $\hat{\Theta}$ is a linear operator so that many solutions can be obtained as a linear combination of the eigenvector solutions.

\subsubsection{Band structure analysis}

Photonic crystals, like atoms or molecules, are invariant for a given translation that is multiple of the structure pitch. Hence, they have a discrete translational symmetry in the periodic spatial direction. The periodic length of the structure is called the lattice constant $a$ and the step vector is the primitive lattice vector. Therefore, for a given structure, its dielectric constant $\epsilon(\mathbf{r})$ will be repeated an integer multiple times $\epsilon(\mathbf{r})=\epsilon(\mathbf{r} \pm a)$. The dielectric unit that forms the photonic crystal along the spatial direction is known as the basic unit cell and its key to identify the eigenmodes of the structure. Furthermore, the solutions of Eqs. 2.3.8 for $\mathbf{H}$ can be expressed as the product of a plane wave with a

\footnotetext{
${ }^{9}$ In related literature it is very common to find eigen as a prefix to define any part of the eigenvalue problem such us the eigenfield, eigenmode or eigenstate.
} 

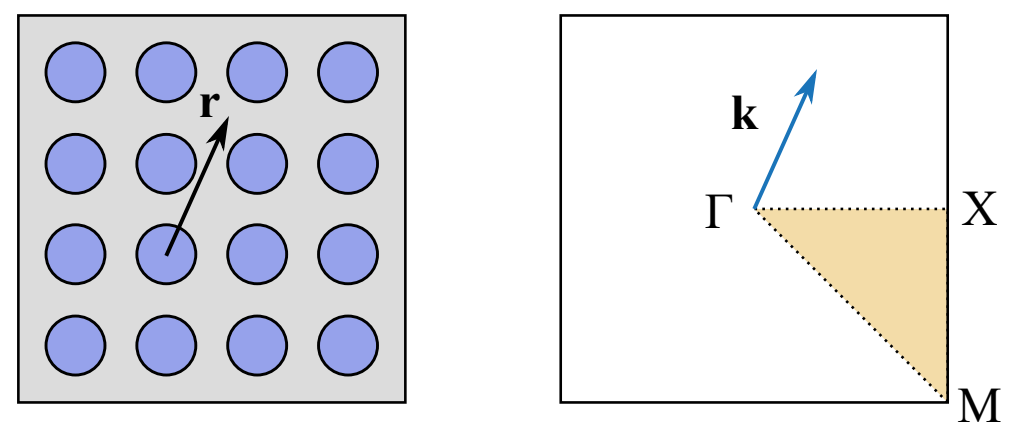

Fig. 2.12 Square lattice of a two-dimensional photonic crystal. On the left hand side the real space is represented and on the right hand side the reciprocal lattice with its corresponding Brillouin zone [60].

periodic function by the following equation

$$
\mathbf{H}_{k}(\mathbf{r})=e^{i \mathbf{k r}} \mathbf{u}_{\mathbf{k}}(\mathbf{r}),
$$

where $\mathbf{k}$ is the wavevector that determines the phase and $\mathbf{u}_{k}(\mathbf{r})$ is a periodic function that defines the field profile with the same periodicity that the dielectric structure, where $\mathbf{u}_{k}(\mathbf{r})=\mathbf{u}_{\mathbf{k}}(\mathbf{r}+\mathbf{R})$ for all lattice vectors $\mathbf{R}$, which in turn, have the same number of components as the $\mathrm{PhC}$ of periodic dimensions. These are known as Bloch modes ${ }^{10}$ due to the similarities with Bloch states in solid-state physics and Floquet's modes in mechanics $[64,65]$. It should be noted that wavevectors that differ an integer multiple times $2 \pi / a$ are physically the same, so that wavevectors only in the range $-\pi / a<k<\pi / a$ must be considered. This region of non-repeated wavevectors, see Fig. 2.12, is called the Brillouin zone ${ }^{11}$. The reciprocal space of wavectors will be also periodic and defined by the reciprocal lattice vectors. In a $3 \mathrm{D}$ photonic crystal with lattice vectors $\left(\mathbf{a}_{1}, \mathbf{a}_{2}, \mathbf{a}_{3}\right)$ their reciprocal lattice vectors are $\left(\mathbf{b}_{1}, \mathbf{b}_{2}, \mathbf{b}_{3}\right)$ and may be calculated as $\mathbf{a}_{i} \mathbf{b}_{j}=2 \pi \delta_{i j}$. These reciprocal lattice vectors are used to calculate a Bloch mode by using the expression $\mathbf{k}=k_{1} \mathbf{b}_{1}+k_{2} \mathbf{b}_{2}+k_{3} \mathbf{b}_{3}$ where $k$ lies in the Brillouin zone.

In order to calculate the band structure of the system and solve the periodic function $\mathbf{u}_{\mathbf{k}}(\mathbf{r})$, we need to insert the Bloch state of Eq. 2.3.13 into the master equation 2.3.8 as

$$
\hat{\Theta} \mathbf{H}_{\mathbf{k}}=\left(\frac{\omega(\mathbf{k})}{c}\right)^{2} \mathbf{H}_{\mathbf{k}}
$$

\footnotetext{
10 The fundamentals of Bloch theorem was discovered independently by Hill, Floquet, Lyapunov and Bloch, but the nomenclature refers to the latest.

${ }^{11}$ More detailed information on the reciprocal lattice and Brillouin zones can be found in [60].
} 
substituting the operator $\hat{\Theta}$ we obtain

$$
\begin{aligned}
& \nabla \times \frac{1}{\epsilon(\mathbf{r})} \nabla \times e^{i \mathbf{k} \cdot \mathbf{r}} \mathbf{u}_{\mathbf{k}}(\mathbf{r})=\left(\frac{\omega(\mathbf{k})}{c}\right)^{2} e^{i \mathbf{k} \cdot \mathbf{r}} \mathbf{u}_{\mathbf{k}}(\mathbf{r}), \\
& (i \mathbf{k}+\nabla) \times \frac{1}{\epsilon(\mathbf{r})}(i \mathbf{k}+\nabla) \times \mathbf{u}_{\mathbf{k}}(\mathbf{r})=\left(\frac{\omega(\mathbf{k})}{c}\right)^{2} \mathbf{u}_{\mathbf{k}}(\mathbf{r}), \\
& \hat{\Theta}_{\mathbf{k}} \mathbf{u}_{\mathbf{k}}(\mathbf{r})=\left(\frac{\omega(\mathbf{k})}{c}\right)^{2} \mathbf{u}_{\mathbf{k}}(\mathbf{r}),
\end{aligned}
$$

where $\hat{\Theta}_{\mathbf{k}}$ is a new Hermitian operator that depends on $\mathbf{k}$ and is defined as

$$
\hat{\Theta}_{\mathbf{k}} \triangleq(i \mathbf{k}+\nabla) \times \frac{1}{\epsilon(\mathbf{r})}(i \mathbf{k}+\nabla) \times .
$$

The last of Eqs. 2.3.15 together with the periodic function $\mathbf{u}_{\mathbf{k}}(\mathbf{r})$ allow $\mathbf{u s}$ to calculate the supported modes by a photonic crystal for each wavevector $\mathbf{k}$. This problem will be limited for the Brillouin zone in the reciprocal space, obtaining infinite solutions for different frequencies ${ }^{12}$. These solutions are ordered in bands with difference indices $n$, so that the $n=0$ band corresponds to the lowest frequency solution of the system. Moreover, these bands continuously evolve with the wavevector, giving rise to the band diagram or dispersion relation of a photonic crystal $\omega_{n}(\mathbf{k})$.

To calculate the band diagram $\omega_{n}(\mathbf{k})$ of a photonic crystal with a given periodic structure $\epsilon(\mathbf{r})$ we need to make use of computational tools that solve the aforementioned equations. In this work we use MPB (MIT Photonics Bands) developed by the Massachusetts Institute of Technology, which is an open source software that computes definite-frequency eigenstates of the latest equation of 2.3.15 in fully-vectorial and three-dimensional spaces. It employs an iterative technique to minimize the solutions of the system for each wavevector $\mathbf{k}$ by using plain wave expansion (PWE) numerical methods ${ }^{13}$.

Figure 2.13 shows an example of band diagram obtained for a two-dimensional photonic crystal consisting of a rectangular array of dielectric columns with a lattice constant $a$. Light propagates within the plane in all directions and for both TE and TM polarizations. The vertical axis represents the frequency of the modes, while the horizontal axis shows the in-plane wavevector $\mathbf{k}$ that moves along the irreducible Brillouin zone, depicted in the left inset of Fig. 2.13. From left to right, it starts from $\Gamma$ to $X$ and then to $M$, covering the entire Brillouin region. Specifically, this band structure has been

\footnotetext{
12 It is worth noticing that, due to rotational symmetries, some of the wavevectors $\mathbf{k}$ inside the Brillouin zone are redundant, thus we only need to calculate those within the smallest region not related with symmetry, the so called irreducible Brillouin zone.

${ }^{13}$ More detailed information about the software and the code can be found in [66].
} 


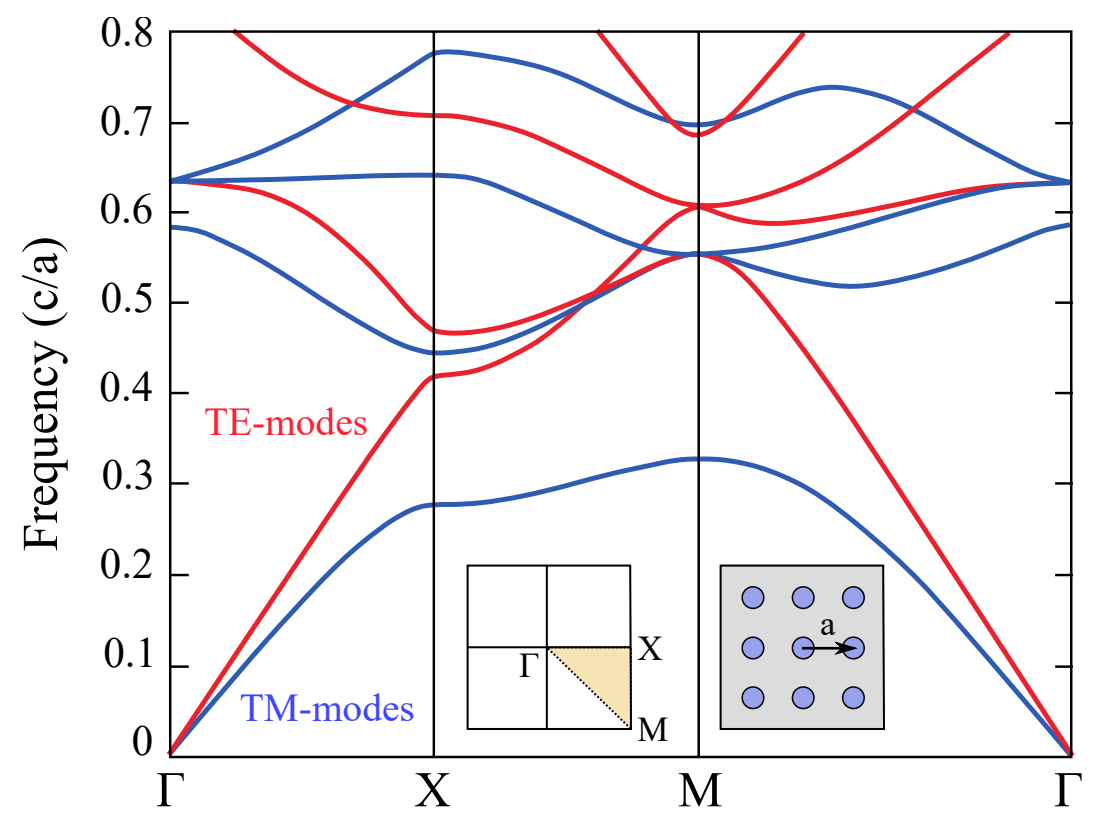

Fig. 2.13 Photonic band structure of a two-dimensional rectangular array of dielectric columns. The red lines represent the TE polarization modes, while the blue ones represent the TM polarization modes. The left inset represents the Brillouin zone with the irreducible area shaded in light yellow. The right inset schematically shows the real space with the dielectric distribution of two dielectric materials represented in different colors [60].

obtained for a crystal structure of alumina ( $\epsilon=8.9)$ rods in air with a given radius of $r / a=0.2$, as it is shown in the right inset of Fig. 2.13.

\subsubsection{Photonic bandgaps and slow light phenomena}

Let us consider a multilayer film as the one shown in Fig. 2.14, forming the simplest photonic crystal in the periodic propagation direction $z$. In the previous section on SWG we have considered light propagation through finely layered media so that we avoid diffraction. In this case, the periodicity is larger enough to produce reflections on each interface and thus a complete band structure analysis is required. The material is periodic in $z$ and homogeneous in the $x y$ plane. Therefore we can distinguish between two different wavevectors parallel and perpendicular to the interface $k_{\|}, k_{z}$, respectively, and the band number $n$. We can express the modes using Bloch formalism as

$$
\mathbf{H}_{n, k_{z}, k_{\|}}=e^{i \mathbf{k}_{\|} \rho} e^{i k_{z} z} \mathbf{u}_{n, k_{z}, k_{\|}}(z)
$$

where $k_{\|}$can be any value since no symmetries can be applied, while $k_{z}$ is restricted within the Brillouin zone $-\pi / a<k_{z} \leq \pi / a$. We now consider waves that propagate only in the $z$ direction so that $k_{\|}=0$. In Fig. 2.14 we show the bands $\omega_{n}(k)$ for the wavevector 
$k=k_{z}$ for three different multilayer cases: where both materials have the same dielectric constant and thus it is actually an homogeneous medium, and for low and high-contrast between the dielectric constants of the layers. Note that for the first case, since there is no real periodicity, the modes lie along the light line given by the refraction index of the material considered. Herein the modes fold back into the Brillouin zone when they reach the limit $k=0.52 \pi / a$ as they are repeated outside this region because of symmetry conditions in the reciprocal lattice. On the other hand, for cases where we have different dielectric constants in the layers and real periodicity, a small gap appears in the region when the modes reaches the end of the Brillouin zone, see the middle and right graph of Fig. 2.14. This is the so-called photonic bandgap, where modes with a frequency within the gap are not allowed to propagate in the crystal. Photonic bandgaps are widely used in many different applications of two and three-dimensional photonic crystals such as narrow-band filters and sensors. Another important fact is that the higher
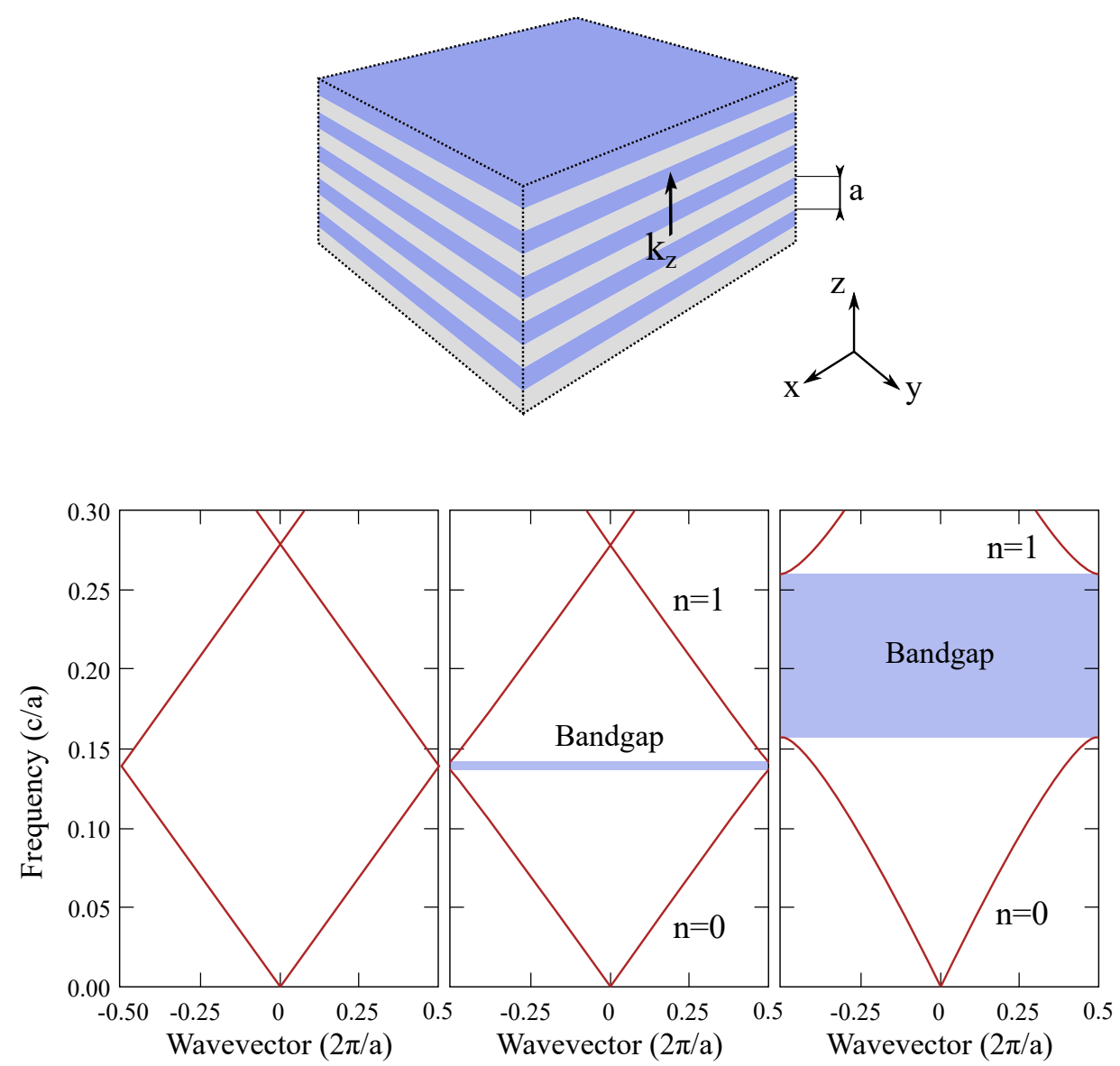

Fig. 2.14 One-dimensional photonic crystal consisting of a multilayer structure and its corresponding band diagram. Three different examples are depicted: on the left graph, both layers present the same dielectric constant $\epsilon_{1}=\epsilon_{2}=13$, in the middle low-index contrast layers of $\epsilon_{1}=13$ and $\epsilon_{2}=12$ and on the right high-index contrast layers of $\epsilon_{1}=13$ and $\epsilon_{2}=1$ [60]. 
the index-contrast between the layers the larger the bandgap. The right graph of Fig. 2.14 shows this effect for a layered medium of $\epsilon_{1}=13$ and $\epsilon_{2}=1$, which produces a remarkable gap of forbidden frequencies.

In the aforementioned examples, we must remark another important effect that arises from the bandgap formation in a photonic crystal. As it is shown in the right graph of 2.14 , both $n=1$ and $n=2$ bands are flattened near the edge of the bandgap. This is strongly related to the group velocity which is just the slope of the band as a function of the wavevector for a given frequency. Mathematically, it is expressed as

$$
v_{g}=\frac{\partial \omega}{\partial k_{z}}
$$

where $k_{z}$ is the wavector in the periodic direction. The group velocity is the velocity that transports the energy in a lossless and non-dispersive medium with a real value of the wavevector. In an homogeneous structure without dispersion, the group velocity is the same than the phase velocity. However, in the case of a photonic crystal, the group velocity can be seen as the velocity of the envelope through the space, and its usually different from the phase velocity ${ }^{14}$. The group velocity is drastically reduced in the vicinity of the bandgap reaching a point where the slope of the band mathematically goes to zero. This region is known as the slow light regime, in which the velocity of the wave can be arbitrarily slowed down to very low values. Moreover, this effect produces a higher interaction between the propagating electromagnetic field and the structure, which is highly desirable for buffering and compressing optical signals for photonic applications [67].

\subsubsection{One-dimensional periodic waveguides}

Three-dimensional photonic crystals can confine light in all spatial directions and provide complete bandgaps for the desired frequency. However, the fabrication of these type of photonic crystals remains challenging due to it structural complexity. Instead of these, simpler structures based on periodic dielectric waveguides are easier to design and fabricate as well as they provide most of the photonic crystal features previously discussed. These structures consist of a one-dimensional periodic pattern in the propagation direction of light in a three-dimensional model with a certain thickness and width. Figure 2.15 depicts some examples of periodic waveguides based on a rectangular waveguide with circular holes, an array of cylinders and a corrugated waveguide, from bottom to top, respectively. All such structures will present a photonic bandgap in the

\footnotetext{
${ }^{14}$ The phase velocity is defined as $v_{p}=\omega / k_{z}$, so that it would be equal to the group velocity in the case that $w$ is directly proportional to $k$
} 


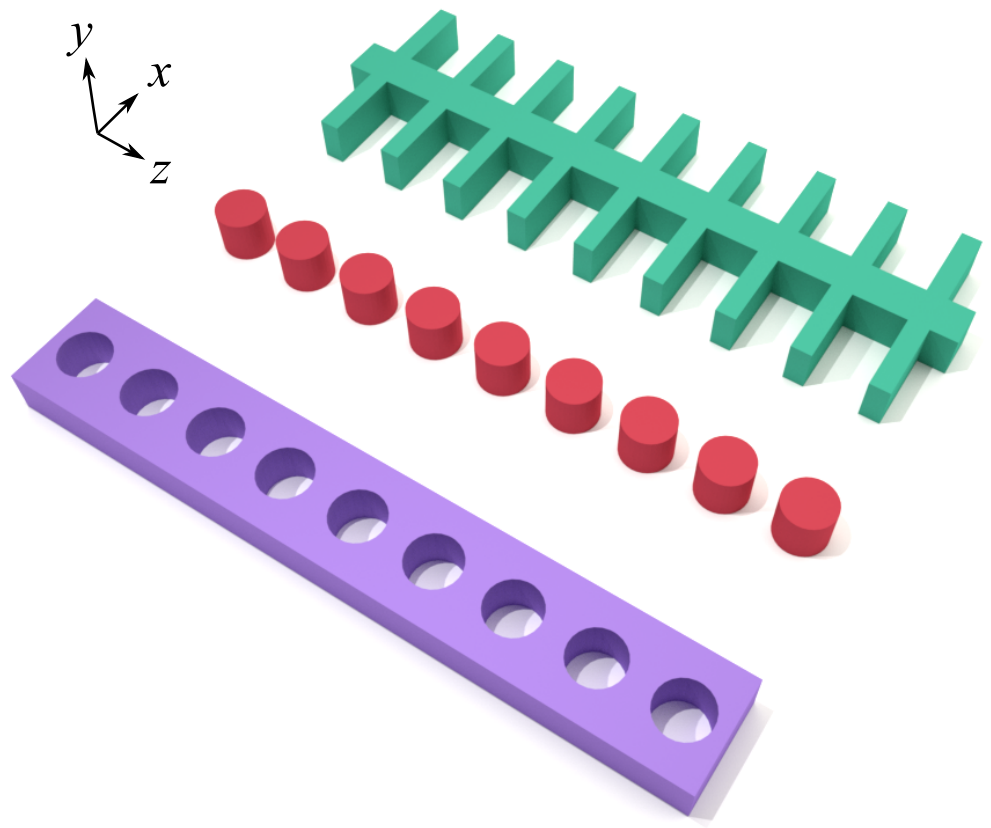

Fig. 2.15 Periodic dielectric waveguides examples consisting of one-dimensional periodic patterns in the propagation direction.

propagation direction due to the periodic pattern, while the light will be confined within the waveguide, in the transversal direction, by the principle of index guiding.

One-dimensional periodic waveguides were first conceived and studied in the middle of the 90s using the band structure analysis by [68-70]. Similar structures, considering an uniform strip waveguide and a periodic set of dielectric squares of $\epsilon=12$, see Fig. 2.16, and herein depicted. In the first case, the homogeneous structure presents a continuous translational symmetry in the $z$ direction so that $k$ is unrestricted in the band study, although here we impose an artificial restriction for the sake of comparison. In the periodic waveguide only the wavector in the periodic direction $z$ is conserved and thus a discrete translational symmetry is obtained. Specifically, dielectric squares with dimensions of $0.4 a \times 0.4 a$ have been considered for the calculations of the bands $\omega_{n}\left(k_{z}\right)$. The wavevector region of $0<k<2 p i / a$ is depicted for both cases in the band structure of Fig. 2.16, as well as the fundamental and first order modes for the TM polarization. The lightcone for $\omega \geq c k$ is also shown for the extended states of light scattered into the air. Below the lightcone, discrete modes exists for both continuous and periodic waveguides, classified as even and odd bands depending on its symmetry in the transversal direction. It should be noted that the first even band corresponds to the fundamental mode that propagates for the lowest frequency. One could think that light propagation in the case of the periodic waveguides results in scattering and losses, however, Bloch theorem states 
that periodic patterns do not need scatter waves since the periodicity guarantees that the wavevector in the propagation direction is conserved. Therefore, the bands shown in the right graph of Fig. 2.16 are truly guided modes and propagate along the waveguide without losses.

Contrary to what occurs in the uniform waveguide, the Brillouin zone in the case of the periodic waveguide is finite. The region $\pi / a<k<2 \pi / a$ is equivalent to $-\pi / a<k<0$ which in turn is a mirrored image of the $0<k<\pi / a$ Brillouin zone region. This fact is key to understand the formation of high-order modes and their interaction in the band diagram depending on the polarization and symmetry. For a wider enough structure, more than two modes can appear for the same frequency resulting in a multimode waveguide. In the following chapters we study how these modes can be engineered to develop bimodal waveguides in periodically patterned structures for high-performance operation. We also show how to exploit the benefits of slow light phenomena in photonic crystals to reduce the length of the device and thus the resulting footprint.

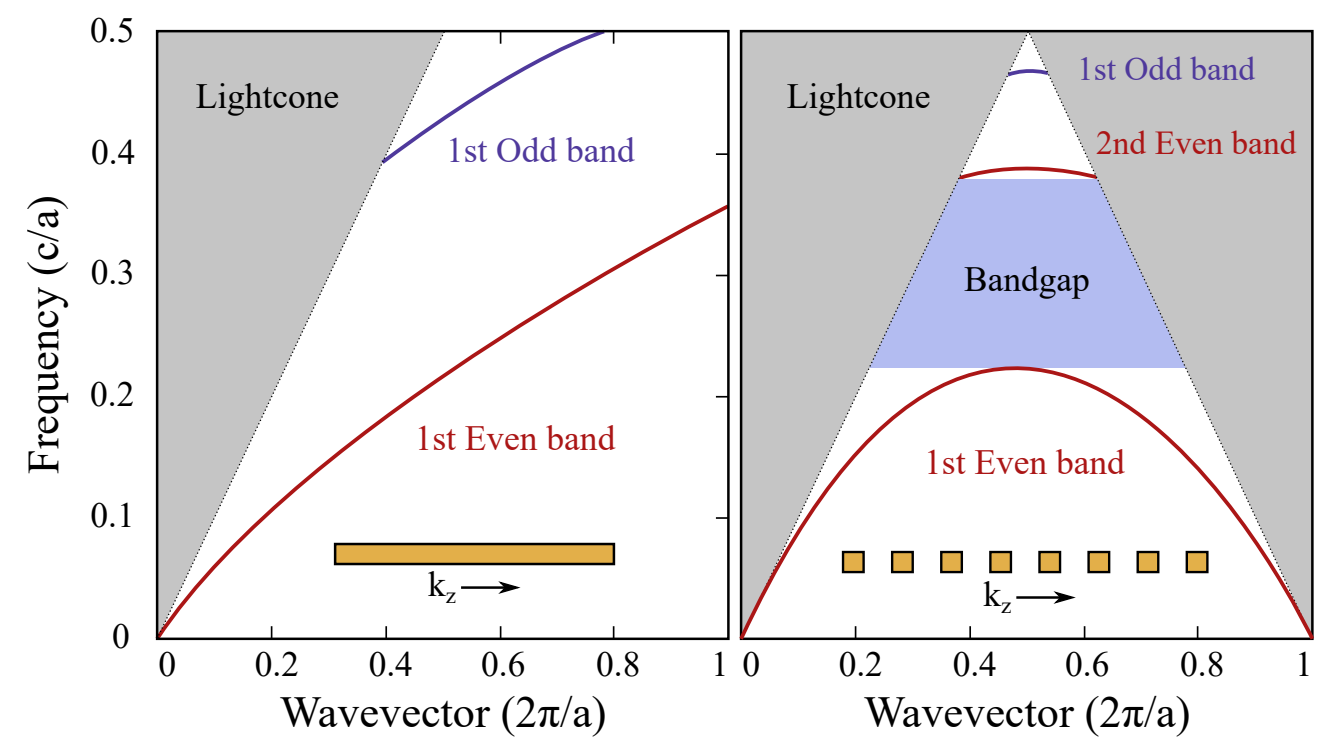

Fig. 2.16 Band diagrams of an uniform and one-dimensional periodic waveguide for the TM polarization, left and right graphs, respectively [60].

\subsection{Bimodal interferometry}

So far, we have seen how light behaves in periodic media depending on the pitch and wavelength of the propagating wave. In this section, we explore integrated interferometers consisting of bimodal configurations based on the previously detailed periodic waveguides. First, we introduce basic concepts of integrated interferometers 
and then we extrapolate them for the case of bimodal periodic platforms where additional effects arise and thus improved performance can be obtained.

\subsubsection{Integrated interferometers}

Optical interferometry is a technique aimed at measuring the interference between electromagnetic waves that propagate along different optical paths [71]. Towards the end of the 19th century, various types of interferometric configurations were discovered as in the case of the Fabry-Perot interferometer, where light passes through parallel reflecting surfaces producing multiple reflections at each interface [72], or the Michelson interferometer, where light is splitted into two optical beams and reflected afterwards to combine their amplitudes [73]. Shortly after, Ernst Mach and Ludwig Zehnder proposed a simpler configuration in which light travels only once through the optical paths, instead of twice as Michelson proposed. This is the so-called Mach-Zehnder interferometer [74, 75], which was widely employed in the next century for all kind of on-chip photonic applications with the revolution of integrated optics [18, 19]. At the same time, common path interferometers were discovered and used for the first time as humidity sensors, where the interference was carried out by two optical modes of the TE and TM polarization. Although both optical modes propagate in the same waveguide, they travel at different velocities because of the polarization dependency, so that a phase shift is induced at the output [76, 77]. More recently, a novel approach of single-channel interferometry was developed by using a bimodal waveguide that supports the first two order modes of the same polarization within an optical waveguide. Similarly to the previous common path interferometer, the higher order mode propagates with a lower propagation constant than the fundamental mode, resulting in an accumulated phase shift over a certain distance [78].

The operation principle of an integrated optical interferometer is shown in Fig. 2.17. On the left hand side a MZI is shown, where light is coupled into the fundamental mode of the input waveguide, which is splitted into two different arms and recombined afterwards in a single output port [79]. In contrast, in a BiM waveguide, see right sketch of Fig. 2.17, a single mode input port supporting the fundamental mode excites the first two order modes of the bimodal part that similarly contribute to the excitation of the fundamental mode at the output. In this case the interferometric performance is carried out in a single-channel structure [31], while in the MZI case, two different physical paths are required. In both approaches, however, a relative phase shift between the optical signals is obtained at the output port as a result of either the physical length difference between the arms in of the MZI, or the propagation constant difference between the modes of the BiM waveguide [80]. 
Due to the wave nature of light, the measured intensity at the output of an interferometer can be mathematically expressed as a sinusoidal function of the phase shift by the following equation

$$
I_{\text {out }}=I_{1}+I_{2}+2 \sqrt{I_{1} I_{2}} \cos (\Delta \phi)
$$

where $I_{1,2}$ are the intensities in the first and second arms in a MZI, or for the first a second modes in a BiM waveguide, and $\Delta \phi$ is the phase shift between both optical signals at the output. The phase shift of the different optical paths $L_{1}$ and $L_{2}$ can be defined as

$$
\Delta \phi=k_{0}\left(\int_{L_{1}} n(r) d r-\int_{L_{2}} n(r) d r\right)
$$

where $k_{0}=2 \pi / \lambda_{0}$ is the wave number, $\lambda_{0}$ the wavelength in vacuum and $n(r)$ the refractive index of the medium at a certain point $r$. Likewise, the phase shift can also be expressed in terms of the effective index of the propagating modes through the interferometer as follows ${ }^{15}$

$$
\Delta \phi=\frac{2 \pi}{\lambda_{0}}\left(L_{1} n_{e f f 1}-L_{2} n_{e f f 2}\right),
$$

where $L_{1,2}$ are the lengths and $n_{e f f 1,2}$ the effective indices of the the first and second arms or modes, in a MZI or in a BiM interferometer, respectively, see Fig. 2.17.

It is worth noticing that $n_{\text {eff } 1}=n_{\text {eff } 2}$ in a MZI when the dimensions and refractive index of both arm waveguides are equal, so that the phase shift will therefore depend

\footnotetext{
${ }^{15}$ The effective index can also be expressed in terms of the propagation constant, or wavevector in the propagation direction, as $\beta=k=n_{\text {eff }} 2 \pi / \lambda$.
}
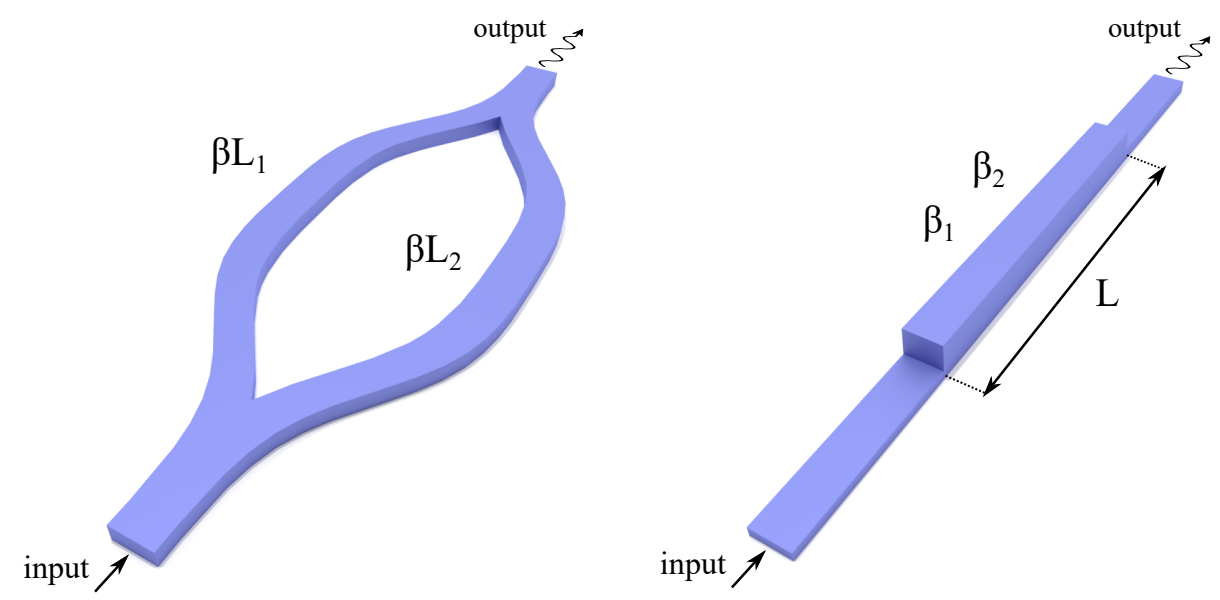

Fig. 2.17 Integrated interferometer examples. A Mach-Zehnder configuration is shown on the left hand side, while a bimodal waveguide is depicted on the right hand side. 
entirely on the length difference between the paths, otherwise they phase shift will be zero. Similarly, in a BiM waveguide, since both modes propagate through the same optical path, $L_{1}=L_{2}$ and hence the phase shift is produced as a result of the difference between the effective indices of the modes.

Furthermore, it is also interesting to explore the performance of the interferometer for a given change either in the refractive index of the core or in the cladding. This allows us to investigate the response of the interferometer when a variation in the system is induced for sensing or modulating purposes, for instance. Mathematically, a change in the refractive index of the system will produce a variation in the effective index of the propagating modes $\Delta n_{e f f 1,2}$. We can express the total amount of phase shift difference relative to an arbitrarily given original state by the following equation ${ }^{16}$

$$
\Delta \phi_{T}=\Delta \phi_{1}-\Delta \phi_{2}=\frac{2 \pi}{\lambda_{0}}\left(L_{1} \Delta n_{e f f 1}-L_{2} \Delta n_{e f f 2}\right) .
$$

In a conventional MZI configuration, one of the arms is supposed to be isolated from changes in the refractive index, the so-called reference arm, so that the phase shift will solely depend on the change in the effective index of the mode propagated through the active arm, $\Delta n_{e f f 2}$, for example, while $\Delta n_{e f f 1}=0$. On the other hand, in a BiM waveguide both modes will interact with the refractive index variations since they propagate along the same path and cannot be isolated from each other. That is the reason why BiM waveguide sensors are designed to maximize the effective index difference, specifically to obtain a large variation in the higher order mode compared to the fundamental one $\Delta n_{\text {eff } 2}>\Delta n_{\text {eff } 1}$. This is due to the fact that the higher order mode is less confined within the waveguide so it is more sensitive to cladding refractive index variations than the fundamental mode, which in turn acts as a reference [31]. It should be also noted that in both cases the resulting phase shift depends on the length $L$ of the interferometer, which explains why the designs are usually that long and why they require very large footprints.

\subsubsection{Bimodal behavior in periodic media}

After revising the fundamentals of conventional interferometers, in this section we will investigate how we can benefit from periodically patterned waveguides to enhance the interferometric response. The idea of merging periodic structures and interferometers was first introduced at the end of the last century [81]. The underlying concept was to reduce the group velocity of one of the propagating modes in a MZI so that the optical path can be increased while the physical length remains small. This is translated

\footnotetext{
${ }^{16} \Delta \phi_{1}$ represents the phase shift for an arbitrarily initial state, and $\Delta \phi_{2}$ is the phase shift after a certain change in the refractive index of the system.
} 
into a higher variation of the effective index $\Delta n_{\text {eff }}$ for a given change in the system refractive index, and consequently in a higher accumulated phase shift, see Eq. 2.4.4. In a non-dispersive material like silicon, engineered structures such as periodic waveguides can produce some dispersion effects in the propagating waves that can be used to improve the interferometer efficiency.

\section{Interferometry in SWG structures}

As we have seen in previous sections, periodic waveguides working in the deep-subwavelength region can be modelled as uniform structures with a pitch much smaller than the wavelength of light. However, as we approximate to the $\mathrm{PhC}$ regime increasing the periodicity, electromagnetic modes become dispersive and the resulting effective index of the effective material becomes strongly dependent on the frequency. In multimode SWG waveguides, the fundamental mode becomes dispersive as it reaches the end of the first Brillouin zone, while the higher order modes are not affected. This fact produces a constant phase shift between the lowest order modes for a wide range of wavelengths, which has been used in reported works to demonstrate broadband operation in SWG configurations.

In evanescent-wave based sensors, hetero-modal interferometers with constant phase shifts as a function of wavelength are investigated for critical spectral-based sensitivity [82]. The operation principle of these devices is based on the interference between two modes with different propagation constants, such as a MZI with different waveguide dimensions in each arm. In these configurations, the output power is measured for a range of source wavelengths, so that the sensitivity expressed in increments or differentials can be defined as

$$
S=\frac{\Delta \lambda}{\Delta n_{c}} \rightarrow S=\frac{\partial \lambda}{\partial n_{c}}
$$

where $\Delta \lambda$ is the shift of a certain spectral feature for a given change in the bulk cladding refractive index $\Delta n_{c}$. Let us now consider that $L_{1}$ and $L_{2}$ are equal and that we work on a spectral peak (constructive or destructive interference), we can rewrite Eq. 2.4.4 as

$$
\Delta \phi\left(\lambda, n_{c}\right) \equiv \frac{2 \pi L}{\lambda} \eta\left(\lambda, n_{c}\right)=N \pi
$$

where $\eta=\Delta n_{e f f 1}-\Delta n_{e f f 2}$ and $\mathrm{N}$ is an integer number, an even value for the case of a constructive interference and odd for the destructive case. Using implicit function differentiation, the phase derivative respect to the cladding refractive index is defined as

$$
\frac{\partial \phi\left(\lambda, n_{c}\right)}{\partial n_{c}}=\frac{2 \pi L}{\lambda} \frac{\partial \eta\left(\lambda, n_{c}\right)}{\partial n_{c}},
$$


and respect to lambda as

$$
\frac{\partial \phi\left(\lambda, n_{c}\right)}{\partial \lambda}=\frac{2 \pi L}{\lambda}\left(\frac{\partial \eta\left(\lambda, n_{c}\right)}{\partial \lambda}-\frac{\eta\left(\lambda, n_{c}\right)}{\lambda}\right)
$$

Dividing Eq. 2.4.7 by Eq. 2.4.8, we obtain an expression for the sensitivity as a function of the phase shift $\phi$, the effective index differences $\eta$ and wavelength $\lambda$ :

$$
S=\frac{\partial \lambda}{\partial n_{c}}=\frac{\partial \phi\left(\lambda, n_{c}\right) / \partial n_{c}}{\partial \phi\left(\lambda, n_{c}\right) / \partial \lambda}=\frac{\partial \eta\left(\lambda, n_{c}\right)}{\partial n_{c}} /\left(\frac{\partial \eta\left(\lambda, n_{c}\right)}{\partial \lambda}-\frac{\eta\left(\lambda, n_{c}\right)}{\lambda}\right)
$$

From this expression, first thing we can highlight is that the spectral sensitivity becomes theoretically infinite when the slope of the phase shift as a function of wavelength is zero. Specifically, this condition is reached at the critical working point, lambda $\lambda_{c}$, occurring at

$$
\frac{\partial \eta\left(\lambda, n_{c}\right)}{\partial \lambda}=\frac{\eta\left(\lambda, n_{c}\right)}{\lambda}
$$

On the other hand, using bimodal SWG waveguides allows to combine the constant phase shifts produced by the dispersion of the fundamental mode with the high light-matter interaction typical of SWG periodic elements, to critically enhance the sensitivity in spectral-based interferometric sensors. In addition, note that the sensitivity expression in Eq. 2.4.9 does not depend on the interferometer length, so that long paths are not required for high performance operation. In order to illustrate this, Fig. 2.18 schematically shows a comparison between a uniform and a SWG bimodal waveguide for the lowest order modes used to perform the interferometry. The upper figures depict the propagation constant of both modes at an arbitrarily initial cladding, solid line, and after a induced change in its refractive index, dashed line. Note that the $\beta_{0}$ mode becomes dispersive for lower wavelengths in the SWG structure, which produces an almost constant phase shift as a function of wavelength, see middle graphs of Fig. 2.18 while in the uniform waveguide the phase shift is directly proportional to the wavelength. The lower graphs show the transmission spectra generated by the phase shift for a certain length. In the uniform waveguide, small spectral shifts of the dips towards higher wavelengths are shown after a change in the cladding refractive index, while in the SWG case the spectral shifts are larger and shifted towards lower and higher wavelengths around the critical working point. In chapter 3 we will see in much more detail the design and development of a refractive index sensor based on bimodal SWG structures, as well as the experimental verification of the spectral sensitivity based on the effects herein showed. 


\section{Interferometry in PhC structures}

In a conventional uniform bimodal waveguide, the high-order mode is usually more sensitive to changes in the refractive index since it is less confined within the core, and hence with a lower effective index than the fundamental mode. For the case of bimodal SWG waveguides, the fundamental mode becomes dispersive, so that both modes are sensitive and somehow they cancel each other, accumulating almost no phase shift at the output. In order to critically enhance the phase shift in a bimodal interferometer, we

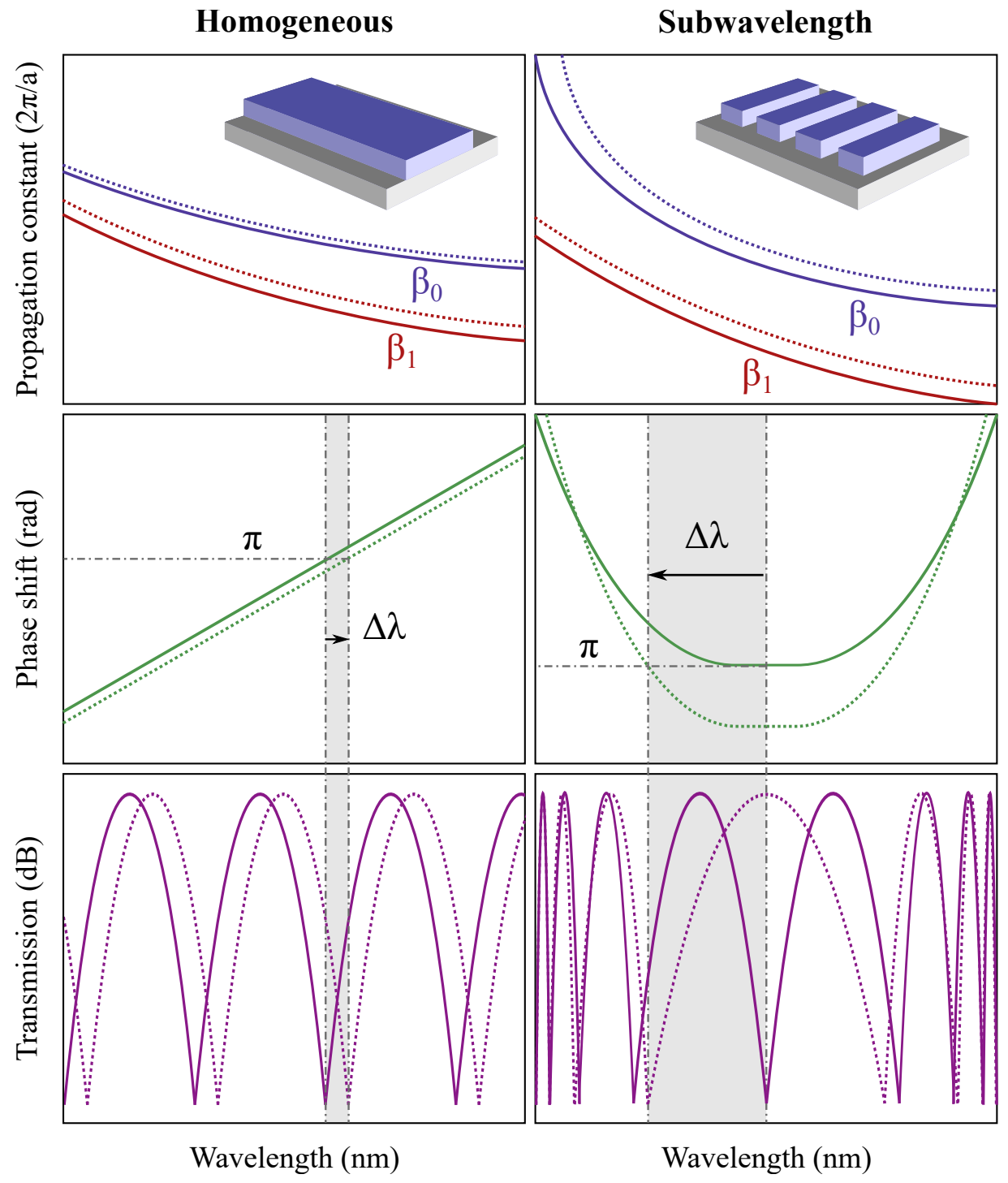

Fig. 2.18 Comparison between the uniform and the subwavelength bimodal waveguides under a static cladding and for a given variation in its refractive index $\Delta n_{c}$, solid and dashed lines, respectively. From upper to lower figures, the dispersion relations, phase shifts and transmitted spectra are shown. 
must reduce the group velocity of the higher order mode (the most sensitive one), and thus increase the change in its effective index when a refractive index variation is induced. On this matter, PhCs allow us to engineer bimodal interferometers with highly-dispersive high-order modes by properly designing the band structure of the unit cell. In this case, the interferometers are exploited to exhibit a large phase shift at the output, similar to a conventional MZI and in contrast to SWG bimodal waveguides, where we focused on the spectral shifts. For sensing purposes, the sensitivity is now expressed in terms of the phase shift as

$$
S=\frac{\Delta \phi}{\Delta n_{c}}=\frac{2 \pi L}{\lambda_{0}}\left(\frac{\partial n_{e f f 2}}{\partial n_{c}}-\frac{\partial n_{e f f 1}}{\partial n_{c}}\right)
$$

where $\Delta n_{c}$ is the change in the refractive of the cladding, and $\Delta n_{e f f 1,2}$ the variation of the fundamental and high-order modes effective index, respectively. As it is expressed by this equation, the higher the difference between the effective index of the modes, the higher the phase shift. By reducing the group velocity of the high-order mode we increase the effective index differences when a change in the cladding is produced. In addition, this conclusion can be extrapolated to changes not only in the cladding but also in the silicon core, to extend the use of the interferometer beyond sensing.

Figure 2.19 illustrates how bimodal waveguides are formed in one-dimensional photonic crystals for larger periodicities than the ones shown with the SWG structures. Here diffraction and reflection effects are not suppressed and photonic bandgaps are therefore produced when the bands reach the end of the first Brillouin zone ${ }^{17}$ [28], see the PBG of the left graph of Fig. 2.19. In this case the unit cell dimensions are not sufficiently large to support more than the fundamental mode, so the entire band diagram is formed by this mode $\beta_{0}$ folded into the Brillouin zone. In contrast, for a $\mathrm{PhC}$ with larger dimensions a second mode $\beta_{1}$ appears and interacts with the fundamental mode in the band formation. In the right graph of Fig. 2.19 the second mode $\beta_{1}$ intersects with the folded fundamental mode $\beta_{0}$, which couple with each other and form an anti-crossing point where no modes are allowed to propagate $[83,84]$. Within this region a second PBG is formed, around which the bands are composed by a mixture of the fundamental and the high-order modes. For the band above the second PBG, a bimodal behavior is obtained, in which the fundamental $\beta_{0}$ mode becomes highly dispersive as it reaches the end of the Brillouin zone. On the other hand, in the case of the band below the second PBG, is the higher order mode $\beta_{1}$ that becomes dispersive as it nears the Brillouin zone edge. This band is of particular interest since the desired bimodal operation previously studied is produced, where the higher order mode works in the slow light regime with very low group velocity values compared to the fundamental mode. Slow light phenomena enhances

\footnotetext{
17 In Fig. 2.19 only the irreducible Brillouin zone is shown although no specific values are given. The limit of the horizontal axis would correspond to a wavevector in the propagation direction, or propagation constant, of $0.5 \pi / a$.
} 
linear a non-linear optical effects as well as enlarge light-matter interactions with the surrounding medium [67]. This effect is visible in Fig. 2.19, when the bands nearing the PBGs present a gentle slope. Since these bands will be shifted when the refractive index is tuned, very large increments in the effective indices are obtained due to the shallow gradient of the modes within the slow light region $[85,86]$.

In chapter 4 we explore the band formation of bimodal PhCs for the creation of high-performance interferometers by reducing the group velocity of the high-order mode to enlarge the optical path while maintaining a short physical length. These effects are investigated for modes of the same even parity and also for the odd parity, specifically to develop refractive index sensors, modulators and switches with drastically reduced footprints.

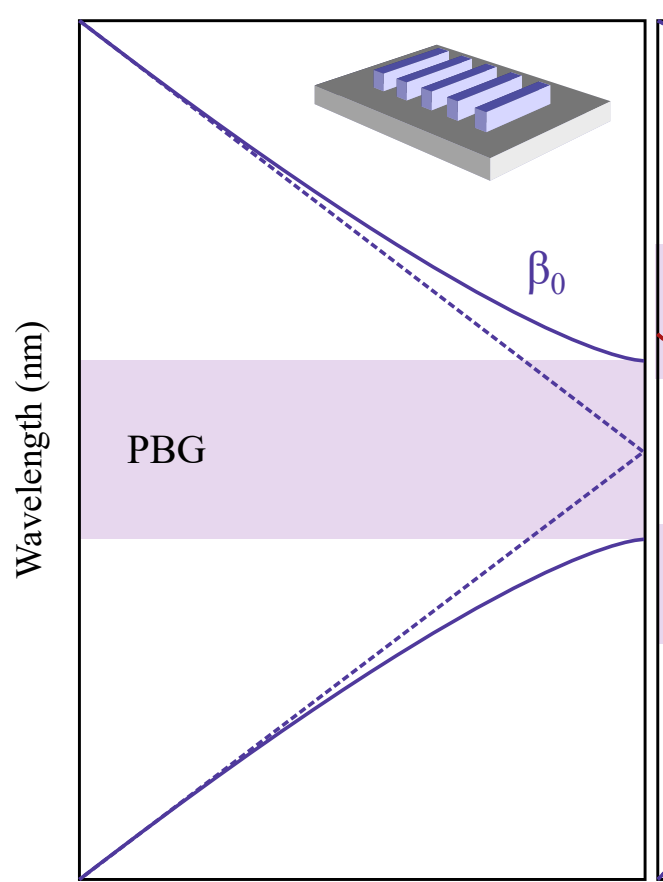

Propagation constant $(2 \pi / a)$

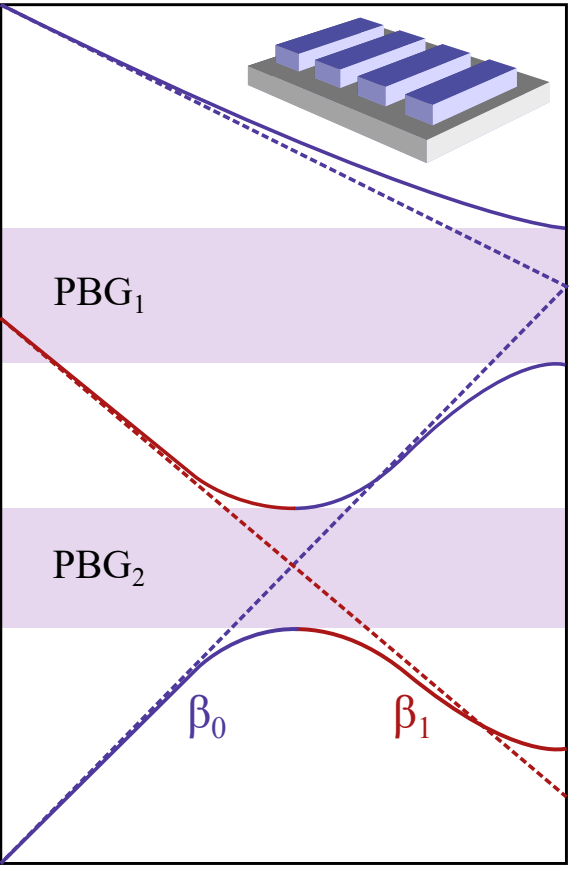

Propagation constant $(2 \pi / a)$

Fig. 2.19 Band structure representation of one-dimensional photonic crystals. The example on the left side hand schematically shows the behavior of the bands for a single mode configuration. On the right side hand, the band structure of a bimodal configuration is depicted. The solid lines show the bands of the periodic structure, while the dashed lines represent the hypothetical bands of a uniform non-periodic waveguide of similar dimensions. 


\section{Chapter 3}

\section{Subwavelength grating bimodal waveguides}

Artificially engineered materials in the subwavelength scale have been known and used for over fifty years in the field of free-space optics [87, 88]. Early studies on subwavelength designs were reported in laminar semiconductor structures with nonlinear optical properties [89, 90], although it was not until the next century when the subwavelength phenomena was implemented in periodic dielectric waveguides [91-94]. Since then, SWG structures, as synthesized materials with a controllable refractive index, have been exploited for a plethora of photonic applications such us input couplers, multiplexers, waveguide crossings or all-optical switches, among others [94-99]. More recently, anisotropy and dispersion effects have been proved in SWGs in order to provide broadband operation in integrated optical devices as beam splitters, multimode interferometers, polarization controllers, mode converters, densely integrated wavguides and gradient-index lenses [100-106]. At the same time, SWG structures have found application in evanescent-wave based sensors as a result of the high field delocalization that interacts with the substance of interest [107, 108]. Due to their the outstanding sensitivty, conventional structures like ring-resonators have been redesigned using SWG waveguides to develop enhanced biosensors with very low limit of detection values compared to other sensing architectures [109-116].

This chapter includes original contributions on subwavelength periodic waveguides for bimodal interferometry in refractive index sensing applications. In Paper A we introduce the theoretical design of a bimodal sensor based on the dispersion effects typical of SWGs for the enhancement of the spectral sensitivity. Paper B address the experimental demonstration and reports a record-high bulk sensitivity value in the state-of-the-art of silicon-based sensors. 



\subsection{Paper A:}

Optics Express 27, 8168-8179 (2019)

\section{Single-channel bimodal interferometric sensor using subwavelength structures}

Luis Torrijos-Morán and Jaime García-Rupérez 



\title{
Single-channel bimodal interferometric sensor using subwavelength structures
}

\author{
Luis Torrijos-Morán and Jaime García-Rupérez \\ Nanophotonics Technology Center, Universitat Politècnica de València, \\ Camino de Vera s/n, 46022 Valencia, Spain
}

\begin{abstract}
A novel configuration of photonic sensor based on a single-channel bimodal interferometer is proposed. The design consists of a subwavelength grating (SWG) periodic structure supporting two dispersive TE-like modes that interfere at the output to create fringes in the transmission spectrum. Dispersion relations of the bimodal periodic structures have been computed in order to study the sensing performance, obtaining a theoretical bulk sensitivity of $\sim 1300 \mathrm{~nm} / \mathrm{RIU}$ and a surface sensitivity of $\sim 6.1 \mathrm{~nm} / \mathrm{nm}$. FDTD analysis has been also carried out to confirm the sensitivity results previously obtained, showing a perfect agreement between theoretical modelling and simulation.
\end{abstract}

\subsubsection{Introduction}

Electromagnetic wave propagation through periodic dielectric media has been widely studied in the literature for a huge range of configurations and applications, among which we can find periodic structures for sensing purposes [1,2]. Within this field, the most relevant examples are photonic crystals (PhCs), which consist of a periodic dielectric repetition in the space for one, two or three dimensions. They were firstly described in detail in [3] as a powerful tool for the implementation of all kind of photonic devices, being later used for a wide range of applications such as modulators, delay lines or filters $[4,5]$. Various types of photonic crystals have been also demonstrated for sensing applications [6-8], offering several advantages in terms of sensitivity and compactness as a consequence of their inherent dispersion properties.

More recently, other type of periodic structures have emerged as a promising alternative in the biosensors field: the so-called subwavelength grating (SWG) structures. These structures can be considered as uniaxial PhCs with lower periodicity values so that the light propagates through the structure as if it were a homogeneous medium, in comparison to the Bragg's behavior occurring in PhCs [9]. Moreover, for periodicity values lower than the wavelength of light, the refractive index (RI) of the resultant metamaterial can be engineered to create photonic devices with enhanced properties. This is the case of broadband beam splitters based on a SWG structure where dispersion control 
was used [10,11], tilted structures controlling anisotropy [12] or high-performance ring resonator (RR) sensors whose sensitivity is around three times that of a conventional RR sensor [13]. When a cladding RI variation takes place, the effective index change of a SWG waveguide mode is significantly higher than for a normal strip waveguide due to the interaction of not only the evanescent field but also the field of the propagating mode itself $[14,15]$. This fact has given rise to the appearance of new photonic sensors based on SWG configurations, offering remarkable advantages respect to the old existing ones and opening new opportunities in this area.

At the same time, integrated interferometers have been commonly used as sensors in lab-on-a-chip (LoC) devices during the last years, with great advantages in terms of sensitivity for label-free detection [16]. Among them, Mach Zehnder Interferometer (MZI) is the most representative configuration $[17,18]$ although it needs from additional structures like MMIs or power splitters to perform the sensing. In addition, different approaches have been reported in the study of interferometric sensors with enhanced sensitivities in spectral interrogation-based sensing [19]. A MZI with different waveguide widths between both arms was experimentally demonstrated for this effect in [20], reporting really high bulk sensitivity values as a consequence of the differential phase shift between the excited modes. On the other hand, single-channel interferometer sensors such as bimodal waveguides appear as one of the best options for integration purposes in LoC devices due to their simplicity and outstanding surface sensitivity for biosensing [21-25]. The sensing principle of these sensors is based on the phase shift produced between the two first TE modes propagating through a bimodal waveguide and measured at the output by a photodetector. However, long bimodal sections are required in order to achieve enough phase shift sensitivity as it scales with sensor length, leading to large footprints of several millimeters or even centimeters.

In this paper, we report the design of a new modal sensor by using a SWG waveguide as a single-channel interferometer. This concept encompasses the benefits of SWG structures in terms of high sensitivity to effective indices variations, while maintaining the characteristics and benefits of bimodal interferometric waveguides. Moreover, as a consequence of working with periodic structures, dispersive modes can be obtained for the SWG structure while behaving like a homogenous medium, what also introduces some of the advantages of PhCs for sensing. As a result, a differential phase shift providing an extremely high spectral sensitivity is obtained, without the need of large paths and additional photonic structures. Band diagrams of the two first TE-like modes were computed to obtain the differential phase shift between them, as well as FDTD simulations to check the spectral response of the sensor under different sensing scenarios, showing bulk sensitivities around $1300 \mathrm{~nm} / \mathrm{RIU}$ and surface sensitivities of $6.1 \mathrm{~nm} / \mathrm{nm}$. This work expands the use of SWG structures for interferometric sensing purposes, 
exhibiting high sensitivities and allowing its integration in reduced footprints of only few microns.

\subsubsection{Operation theory}

When an object structure is larger than the wavelength of light, its electromagnetic behavior can be modelled by the Snell's law using reflection, diffraction and refraction coefficients. Wave propagation through a periodic repetition of this kind of structures gives rise to a lossy system where no light is guided as a consequence of scattering effects [26]. Conversely, if we consider a periodic medium whose lattice constant is much smaller than wavelength, light propagates through the structure as if it were a homogenous medium where diffraction effects are suppressed, thereby obtaining a SWG configuration. Between these two regimes, when the periodicity is not small enough to avoid diffraction, rigorous solution of Maxwell's electromagnetic equations is needed to describe the optical properties of the resultant structure: a PhC. The most common way to delimit these regions and to determine whether we are working in the SWG regime or not is described by the expression $\lambda / \Lambda>2 n_{\text {eff }}$ where $\lambda$ is the operating wavelength and $\Lambda$ is the periodicity value [27].

Here, we work with periods close to the SWG limit and near the PhC zone, where the periodic structure supports two true lossless modes as a consequence of working in the subwavelength regime but with some of the dispersion properties typical of periodic structures. This fact generates non-linear phase shifts falls between both modes that cannot be obtained with a normal bimodal waveguide. The design of the proposed sensor is presented in Fig. 1. It is composed by a SWG bimodal section with a width enough to support two guided TE-like modes with different symmetry in the ' $x$ ' axis (even and odd) for the wavelength range of interest. By properly placing the single mode waveguide used as input port, both modes of the SWG section are excited. After propagating through the bimodal section, these two modes will interfere and contribute to the excitation of the fundamental mode of the single mode waveguide used as output port. The output power will therefore depend on the phase shift between both modes, creating constructive or destructive interferences when the differential phase is an even or an odd multiple of $\pi$, respectively. These interferences will give rise to the appearance of spectral dips in the transmission spectrum for those wavelengths where the modes interfere destructively.

The wavevector difference between the SWG modes for a given cladding RI can be expressed as $\Delta k_{n 1}=k_{1}-k_{2}$ where $k_{1,2}$ are the wavevectors in the propagation direction of the even and odd modes, respectively. When a RI variation is produced in the cladding of the SWG sensing structure, the differential phase shift between both modes is described by the following equation: 


$$
\Delta \phi=L\left|\delta k_{1}-\delta k_{2}\right|
$$

where $L$ is the length of the SWG bimodal section and $\delta k_{1,2}$ are the wavevectors differential for a small change in the cladding RI. Taking into account the dispersion properties of the guided modes, non-linear phase shifts as a function of wavelength are obtained. Consequences of this effect are studied by calculating the dispersion diagrams of the periodic structures and how they behave for changes of the cladding RI. In the following sections, we study how to benefit from dispersion effects in a bimodal SWG interferometric structure to enhance the spectral sensitivity.

\subsubsection{Sensitivity analysis}

\section{Bulk sensing}

In spectral interrogation based sensors such as resonators, the sensitivity is measured from the spectral shift suffered by the spectral features of interest when a change in the

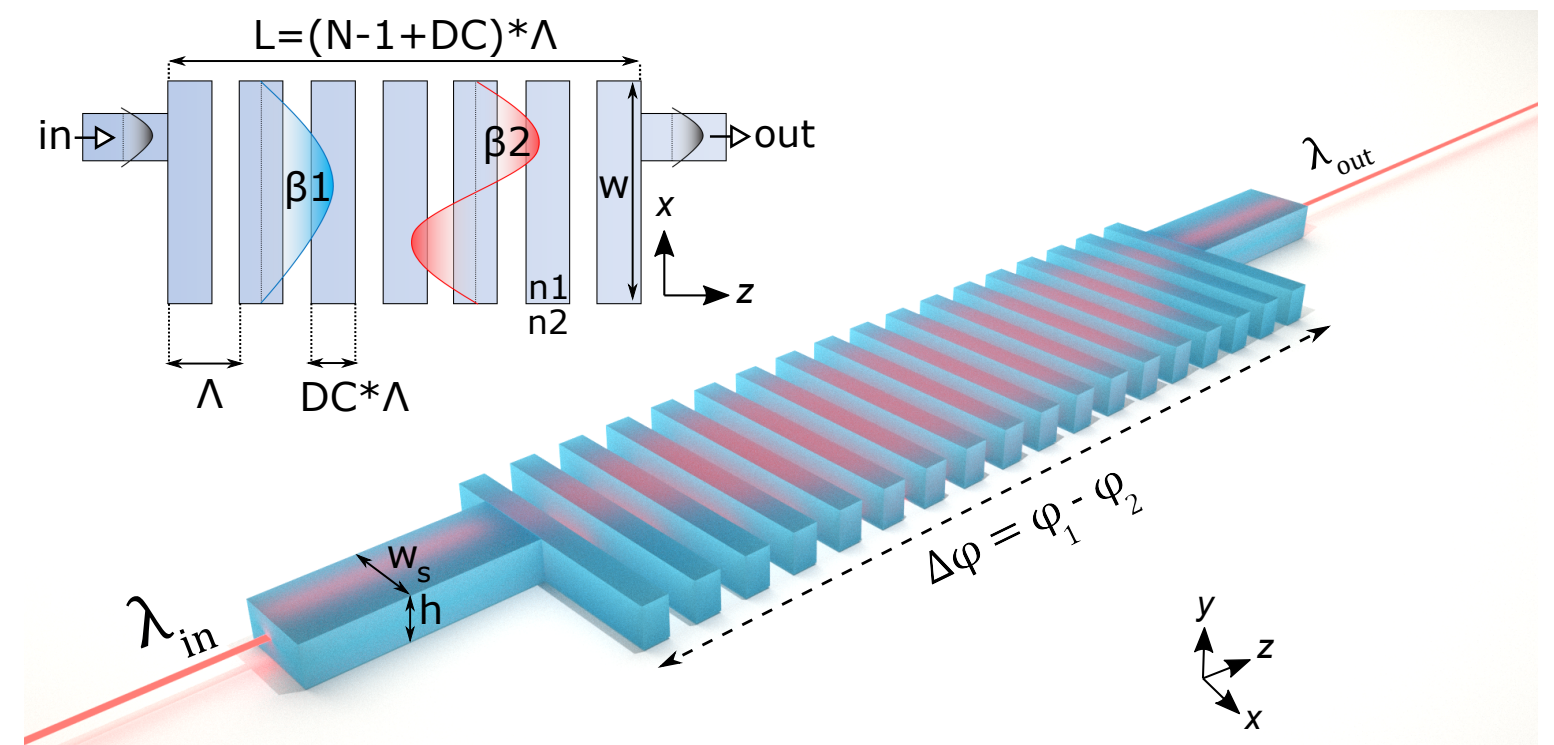

Fig. A.1. Schematic representation of the proposed SWG bimodal sensor where ' $\Lambda$ ' is the period, ' $D C$ ' the duty cycle of the SWG elements, ' $w$ ' the width of the SWG elements, ' $h$ ' the height and ' $w_{s}$ ' the width of the input and output waveguides. The length ' $L$ ' is determined by the number of elements $N: L=(N-1+D C)^{*} \Lambda$. The inset schematically shows the profile of the propagating modes. 
refractive index of the cladding is produced [28]. This type of sensors can define its bulk sensitivity as [29]:

$$
S_{b}=\frac{\delta \lambda_{f}}{\delta n_{c}}
$$

where $\delta \lambda_{f}$ expresses the shift in $\mathrm{nm}$ of a spectral feature for a differential change in the cladding RI $\delta n_{n}$ measured in refractive index units (RIU). In this type of spectral interrogation based sensors, it has been demonstrated both theoretically and experimentally that significantly higher spectral shifts are obtained when using SWG structures, since a dramatic change in the modes propagation constant is produced [13]. These sensing benefits of SWG-based sensors do not only lie in a stronger mode delocalization, but mainly in the direct interaction of the optical field with the target medium in the gaps of the SWG elements.

By multiplying and dividing the expression in Eq. (2) by $\delta \phi$, we obtain an analytical expression for the bulk sensitivity as a function of the phase shift between the even and odd modes of the SWG bimodal sensor:

$$
S_{b}=\frac{\delta \phi / \delta n_{c}}{\delta \phi / \delta \lambda_{f}}
$$

As it can be seen, the sensitivity is inversely proportional to the phase derivative with respect to the wavelength. Because of this, the bulk sensitivity can be analytically infinite as long as the slope of the phase as a function of wavelength reaches zero. This critical lambda point appears for those values where $\delta \phi / \delta \lambda_{f}=0$, as it is reported in [19], and consequently, higher sensitivities are achieved as we get closer to this working point.

Several simulations using MIT Photonics Band (MPB) free software have been carried out in order to obtain the electromagnetic modes of the periodic SWG sensing structure and their dispersion relations. This software computes definite-frequency eigenstates of Maxwell's equations in fully-vectorial and three-dimensional spaces, calculating dispersion relations of the periodic structures for different wavevector points [30]. We have considered a SWG structure made of silicon $(n=3.47)$ over a silica substrate $(n=1.44)$ and an aqueous environment as upper cladding $(n=1.36)$, for which two TE-like modes are supported for an operation wavelength around $1550 \mathrm{~nm}$ when the design values are $\Lambda=290 \mathrm{~nm}, D C=50 \%, w=1400 \mathrm{~nm}$ and $h=220 \mathrm{~nm}$. Due to scaling properties of periodic configurations, the bimodal region can be shifted to other operating wavelengths by properly selecting the period of the structure. The band diagrams obtained for the even and odd TE-like modes of this SWG structure are shown in Fig. 2(a), while Fig. 2(b) shows the phase shift between these two modes as a function of wavelength for different cladding RIs. 
(a)

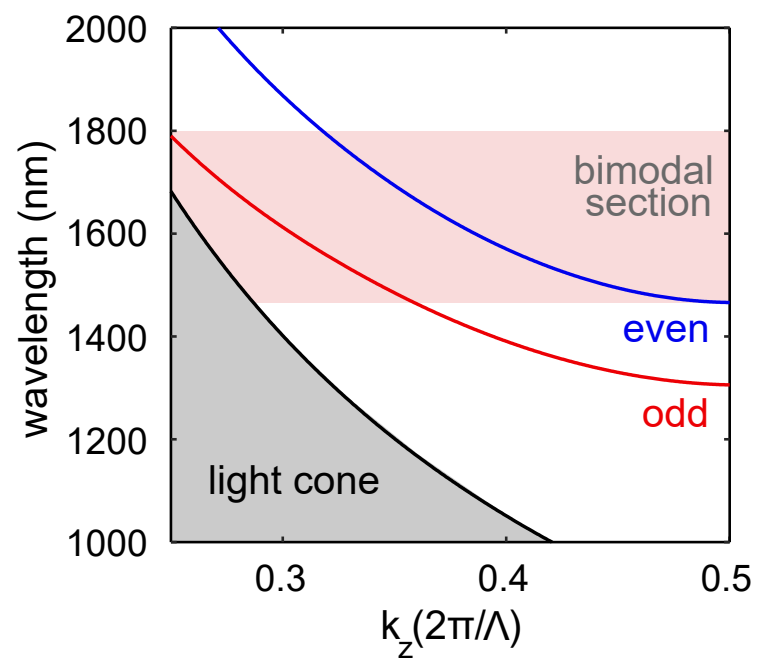

(b)

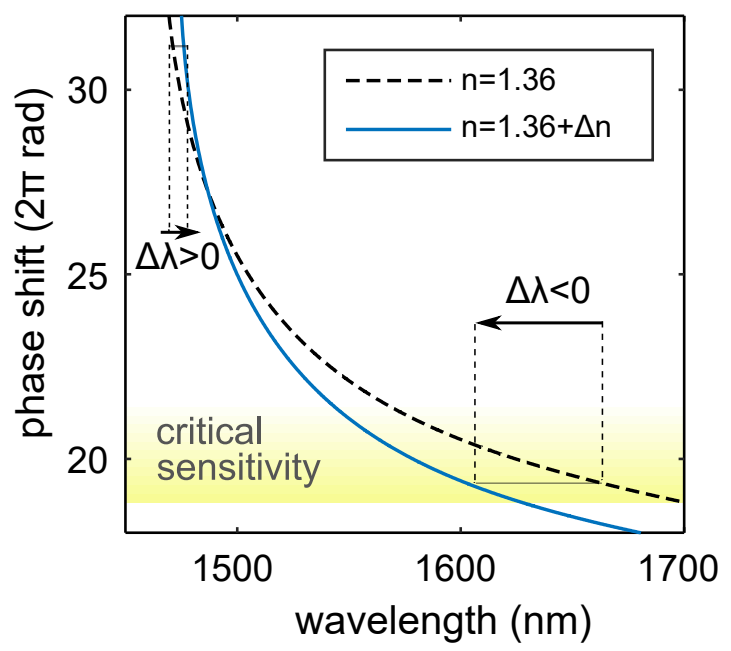

Fig. A.2. (a) Dispersion relations of the even and odd TE-like modes of a SWG bimodal structure of period $\Lambda=290 \mathrm{~nm}, D C=50 \%, w=1400 \mathrm{~nm}$ and $h=220 \mathrm{~nm}$, under an aqueous environment as cladding $(n=1.36)$. (b) Phase shift as a function of wavelength between both modes for a cladding of $1.36 \mathrm{RIU}$ and for a RI change of $\Delta n=0.07 \mathrm{RIU} ; N=40$ periods have been considered.

The first thing we can highlight from Fig. 2(b) is that the sign of the wavelength shift changes from positive to negative values as we move towards higher wavelengths, obtaining a zero-sensitivity point between these two regions for which the phase remains the same regardless the RI of the cladding. We can also observe that an abrupt fall in the phase shift with respect to the wavelength is produced for lower wavelengths, what is determined by the highly dispersive behavior of the even and odd modes near the edge of the Brillouin zone, as shown in Fig. 2(a). Contrary for higher wavelengths, the group velocity of the odd mode increases and starts becoming non-dispersive, provoking a change in the trend of the phase shift and therefore to the appearance of lower slopes as a function of wavelength. Taking into account this fact, the maximum sensitivity appears for the spectral fringes located in the critical sensitivity zone, which is defined as the highest wavelength spectral region where the bimodal condition is obtained, in which the phase slope is close to zero, as it was determined in Eq. (3). Note that, for even higher wavelengths, the odd mode goes below the light cone of the silica lower cladding and becomes a leaky mode, so this spectral region is not considered for the operation of the SWG bimodal interferometer sensor. Finally, we can also highlight that the odd mode presents a lower effective index than the even mode, thus having a higher interaction with the surrounding medium since it is less confined.

The influence of the duty cycle over the phase shift is depicted in Fig. 3(a) under an aqueous environment of $1.36 \mathrm{RIU}$ and when a RI increment of $0.01 \mathrm{RIU}$ is considered. It can be seen how the wavelength shift increases with the duty cycle due to the reduction 
of the slope of the phase shift as a function of wavelength for higher duty cycles. However, additional propagating modes might be supported by the SWG structure when the duty cycle is increased, thus losing the bimodal condition required for the operation of the interferometric sensor. In addition, critical sensitivity regions are narrower for higher duty cycles, making it more difficult to design spectral dips at those zones. Therefore, a trade-off is produced between sensitivity and proper operation, obtaining good results for average values of the duty cycle.

(a)
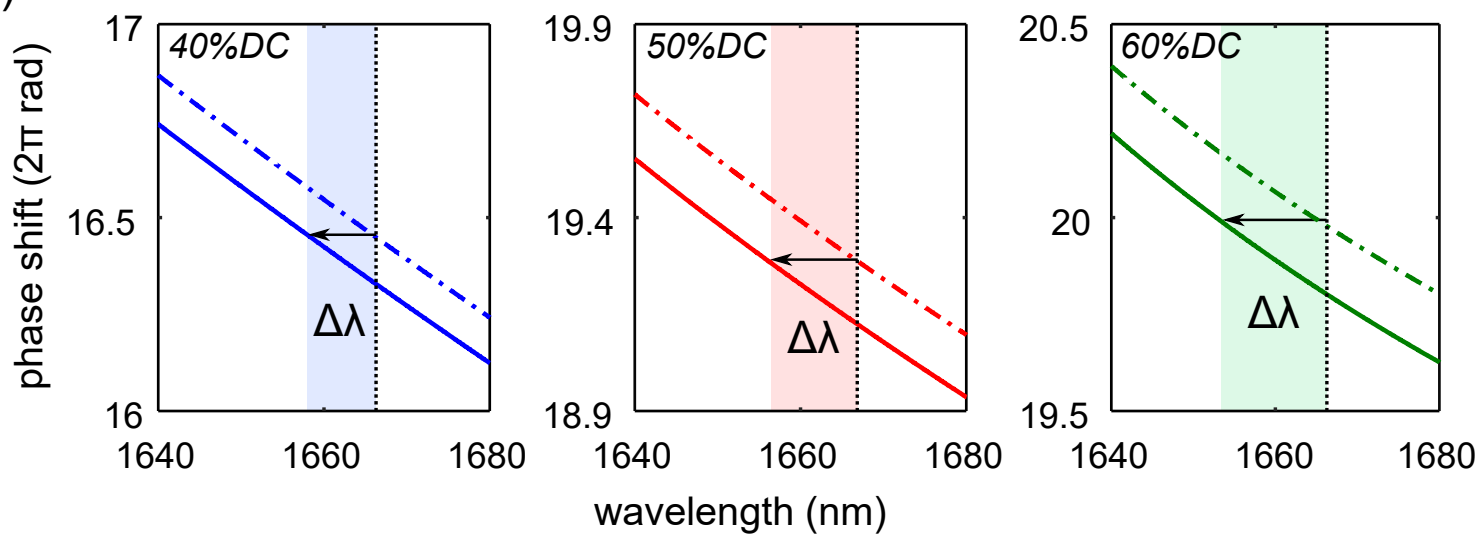

(b)

(c)
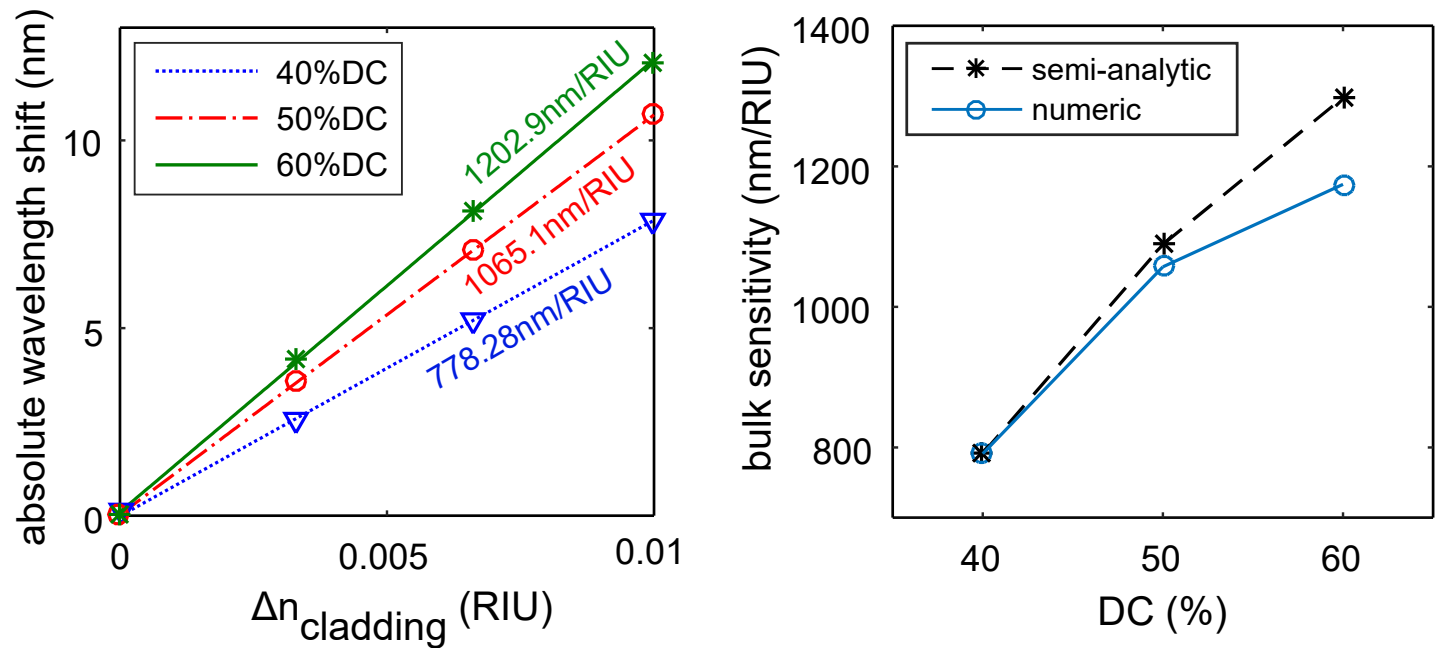

Fig. A.3. (a) Phase shift between both modes of the SWG bimodal structure for different duty cycles. Dashed and solid lines show the phase shift for a cladding RI of 1.36 RIU and 1.37 RIU, respectively. Design parameters are $\Lambda=290 \mathrm{~nm}, D C=50 \%, w=1400 \mathrm{~nm}$ and $h=220 \mathrm{~nm}$. (b) Numerical wavelength shift as a function of the cladding RI for different duty cycles at 1665 $\mathrm{nm}$. Note that the spectral features located in the critical sensitivity region are shifted towards lower wavelengths for increments of the cladding RI, so absolute values of the wavelength shift are considered. (c) Bulk sensitivity comparison between the numerical and the semi-analytic calculations obtained using Eq. (3) at an operation wavelength of $1665 \mathrm{~nm}$. 
Figure 3(b) depicts the sensitivity curves for the different duty cycles considered in the study. They have been numerically calculated from the MPB band diagrams by obtaining the absolute value of the wavelength shift between both modes when considering cladding RI variations being multiple of $3.3 \times 10^{-3}$ RIU. An operating wavelength of $\lambda=$ $1665 \mathrm{~nm}$ has been considered to calculate the sensitivity, since it is within the critical sensitivity range and close to the light cone for the considered design. Bulk sensitivity values of $778.28 \mathrm{~nm} / \mathrm{RIU}, 1065.1 \mathrm{~nm} / \mathrm{RIU}$ and $1202.9 \mathrm{~nm} / \mathrm{RIU}$ have been numerically obtained for duty cycles of $40 \%, 50 \%$ and $60 \%$, respectively. In comparison with the literature, a sensitivity of $490 \mathrm{~nm} /$ RIU was experimentally obtained using a SWG RR [13]. This sensitivity value was recently enhanced by using a multi-box SWG RR, presenting bulk sensitivities of $580 \mathrm{~nm} / \mathrm{RIU}$ [31]. Therefore, our proposed sensor configuration theoretically shows bulk sensitivities more than twice of that reported for other spectral interrogation based SWG sensors, what represents a significant step forward within this type of structures. Note that similar sensitivities to those calculated for our bimodal SWG sensor have been also obtained for other configurations, as for example when using slot waveguide RRs [32]. However, the fabrication of this type of structures is significantly more challenging than for the relatively simple bimodal SWG configuration proposed in this work.

Additionally, semi-analytical bulk sensitivities have been also calculated using Eq. (3) by applying finite differential derivatives, with respect to the cladding RI and the wavelength, on the phase shifts obtained in MPB. A comparison between the numerical and semi-analytical results are presented in Fig. 3(c), showing a good agreement for the different duty cycles considered. However, the difference between numerical and semi-analytical results increase with the duty cycle, obtaining a sensitivity as high as 1298 $\mathrm{nm} / \mathrm{RIU}$ for the semi-analytical study in the case of a $D C$ of $60 \%$. Finally, it should be noted that the bulk wavelength sensitivity does not directly depend on the sensor length if we are measuring the spectral shift. By increasing the sensor length, we can increase the number of fringes appearing at the same spectral range, but the wavelength shift of each spectral feature will remain the same.

\section{Surface sensing}

The surface sensitivity relates the shift of a given spectral feature with the thickness of a certain layer with a different RI than the cladding, considering what happens when a certain biological substance is deposited over the sensor. In the literature, it is defined in $\mathrm{nm} / \mathrm{nm}$ by the following expression for spectral interrogation based sensors [33]:

$$
S_{s}=\frac{\Delta \lambda_{f}}{\rho_{\text {layer }}}
$$


where $\Delta \lambda_{f}$ is the feature spectral shift and $\rho_{\text {layer }}$ is the thickness of the deposited layer. The surface sensitivity has been numerically calculated using MPB for several thicknesses of a layer deposited over the SWG sensing structure and considering an operation in the critical sensitivity region. Figure 4(a) shows the surface sensitivity results obtained for different duty cycles as a function of the layer thickness, while Fig. 4(b) depicts the surface sensitivity as a function of the wavelength for a SWG structure of $60 \%$ duty cycle when the thickness of the deposited layer increases from $10 \mathrm{~nm}$ to $100 \mathrm{~nm}$. Note that since high wavelength shifts of the bimodal region are obtained when different layers are considered, we cannot obtain a common operating wavelength for all thicknesses. Therefore, we have considered as operating wavelengths those where maximum surface sensitivity values are placed. Hence, in order to obtain the maximum surface sensitivity results as possible in future designs, the operating wavelength will depend on the thickness of the specific biolayer to be detected.

A maximum surface sensitivity of $6.138 \mathrm{~nm} / \mathrm{nm}$ has been obtained for a layer thickness of $60 \mathrm{~nm}$ in the case of $60 \%$ duty cycle. As it was explained before, results are strongly dependent on the duty cycle, considerably enhancing the surface sensitivity by a factor

(a)

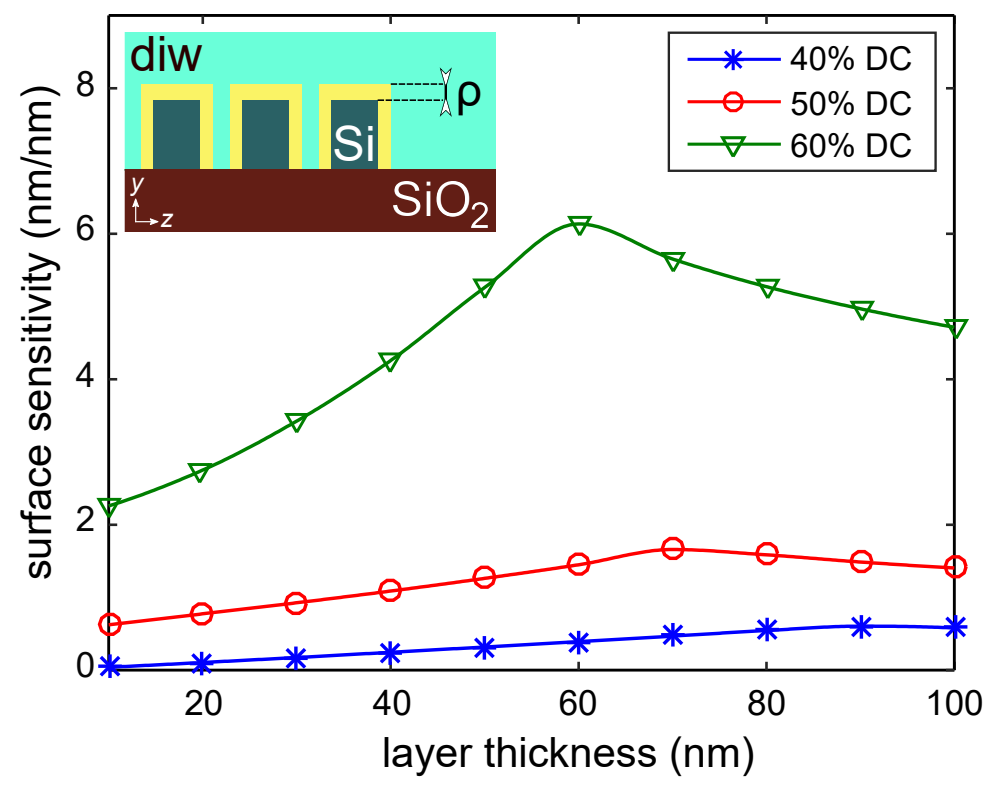

(b)

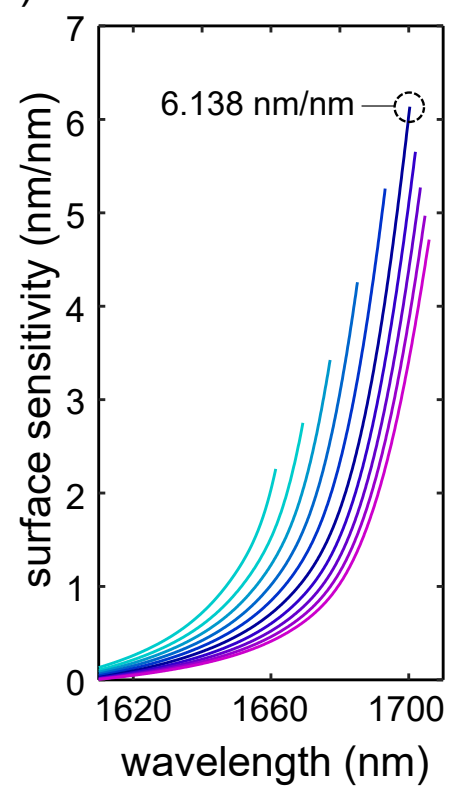

Fig. A.4. (a) Surface sensitivity as a function of the layer thickness $\rho$ for the SWG bimodal sensor in an aqueous environment for different duty cycles. The inset depicts the cross section of the transversal SWG elements in the $y-z$ plane with the deposited layer. A RI of 1.48 RIU is considered for the deposited layer. (b) Surface sensitivity as a function of wavelength for different layer thicknesses and a duty cycle of $60 \%$. The layer thickness goes from $10 \mathrm{~nm}$ (light blue) to $100 \mathrm{~nm}$ (purple). Note that sensitivity values have been calculated considering only wavevectors for which the modes remain above the light cone of the silica lower cladding. 
higher than three in the case of $60 \%$ compared to the case of $50 \%$. This is due to the significant reduction of the phase slope obtained for duty cycles above $50 \%$, as it was previously shown in Fig. 3(a). On the other hand, surface sensitivity decays for certain thicknesses due to the overlap of the deposited layer within the gap of the SWG elements. These outstanding results are obtained thanks to the direct interaction of the optical modes with the deposited layer, obtaining maximum sensitivity values when the hole is totally filled with the target substance. Such big layers around 60-70 nm could be interesting in biosensing applications, for example, in the recognition of viruses or other biological substance with similar dimensions. Nonetheless, the detection of small protein layers of few nanometers is still feasible using this sensor, since more than 2 $\mathrm{nm} / \mathrm{nm}$ of surface sensitivity is obtained for a thickness of $10 \mathrm{~nm}$, making it a promising configuration for biosensing purposes.

Revising the literature, surface sensitivity values around $0.4 \mathrm{~nm} / \mathrm{nm}$ have been previously presented for a conventional RR sensor working for TM polarization; these values have been recently increased until $1 \mathrm{~nm} / \mathrm{nm}$ and $2 \mathrm{~nm} / \mathrm{nm}$ for a SWG RR and for a multi-box SWG RR, respectively, all of them for small layer thicknesses around $10 \mathrm{~nm}$ [34]. Our results show better surface sensitivities for this thicknesses range when considering a $60 \%$ duty cycle and an ultrahigh sensitivity behavior for layers around $60 \mathrm{~nm}$, compared to the values below $0.5 \mathrm{~nm} / \mathrm{nm}$ reported in the multi-box SWG RR for thicker layers.

\subsubsection{Spectral analysis}

\section{SWG bimodal sensor excitation}

FDTD simulations have been carried out using CST Studio software with the aim of analyzing the sensor response when it is excited using the single mode access waveguides. In order to properly excite both modes, an asymmetric coupling of the single mode waveguide in the $x$ axis is needed. The field distribution of the even and odd modes of the SWG structure, calculated by using RSOFT software, is depicted in Fig. 5(a) as well as the profiles of the modes in Fig 5(b). It can be seen that both modes are confined inside the silicon elements of the SWG structure in the case of the $x$ component of the electric field. However, high values of the $z$ component distribution are located within the gaps, what explains the strong field interaction with the cladding. From Fig. 5(b) we can observe that the $x$-axis of the even mode is strongly localized in the center of the SWG structure, while the odd mode is distributed on its outer sides. So, in order to properly excite both modes, the single mode access waveguide has to be displaced respect to the center of the SWG structure ( $x=0$ position). Figure 5 (c) shows the transmission spectra calculated for a SWG bimodal sensor with a duty cycle of $50 \%$ when different lateral positions of an access 
(a)
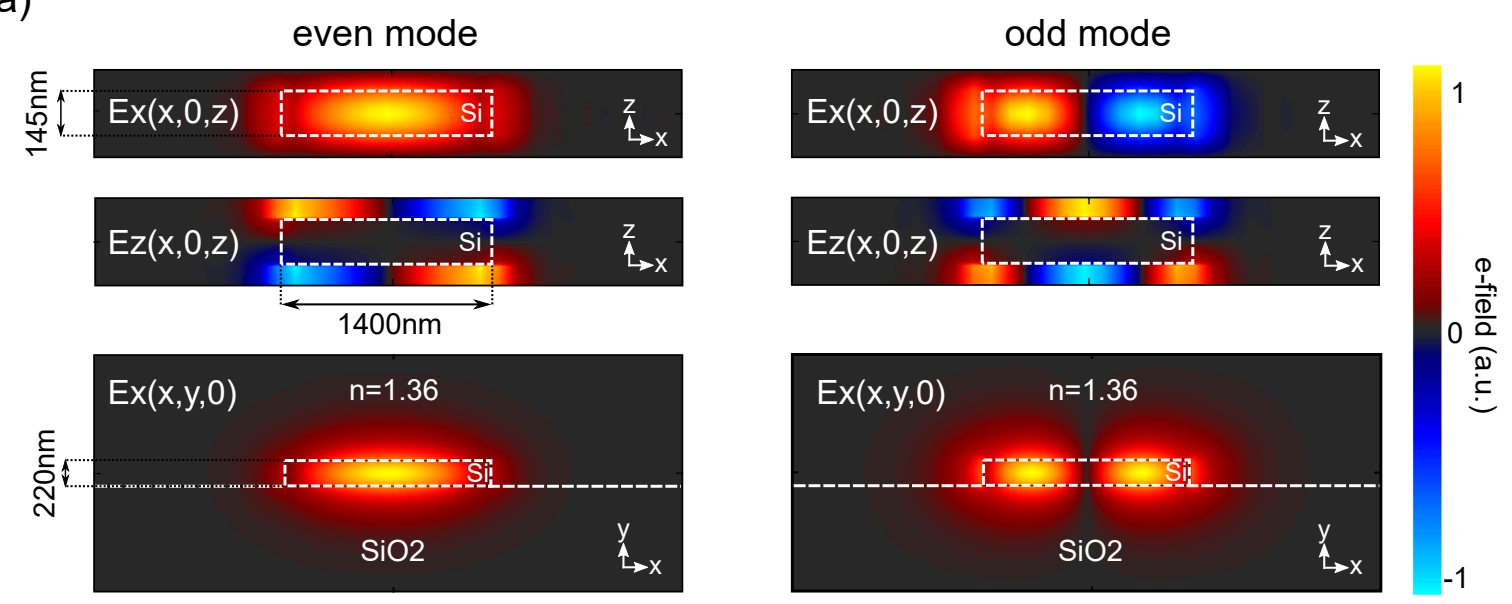

(b)

(c)
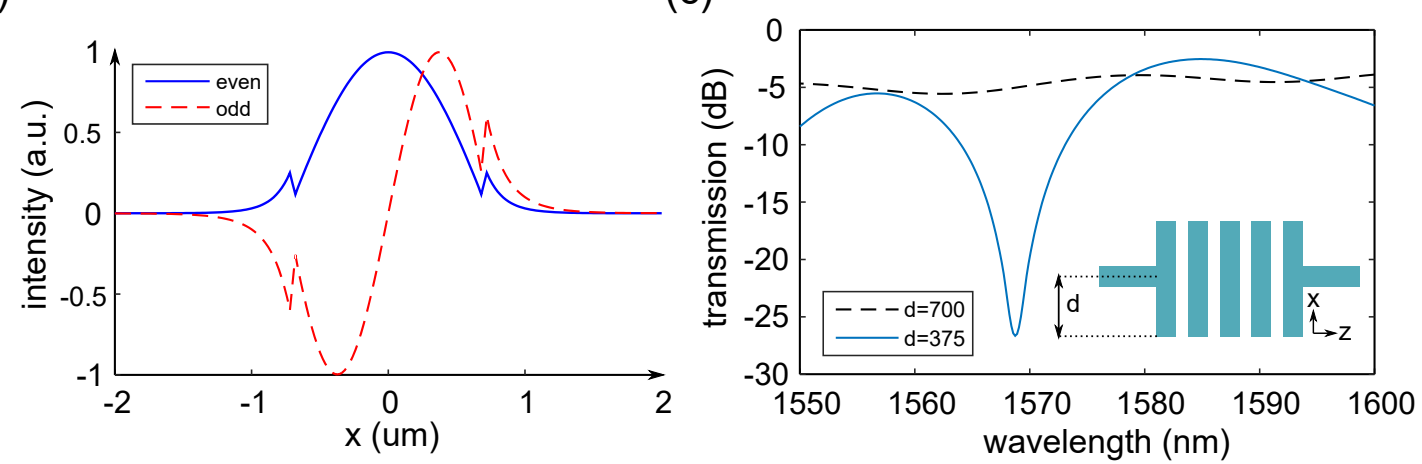

Fig. A.5. (a) Electric field magnitude distribution of a periodic cell of the SWG structure in different planes for even and odd modes. The white dashed rectangles represent the SWG element. Design parameters are $\Lambda=290 \mathrm{~nm}, D C=50 \%, w=1400 \mathrm{~nm}$ and $h=220 \mathrm{~nm}$ for a silica lower cladding and an aqueous environment of $1.36 \mathrm{RIU}$. (b) Mode profile of the $x$-component of the electric field for both modes in the $x$-axis at $y=0$ and $z=0$. (c) Transmission spectra for a $50 \%$ duty cycle SWG bimodal structure as a function of the displacement ' $d$ ' of a single mode waveguide of width $w_{s}=450 \mathrm{~nm}$ at the input and output. A length of $N=120$ elements has been considered for the calculations.

waveguide of width $w_{s}=450 \mathrm{~nm}$ are considered (centered access waveguide $-d=700$ $\mathrm{nm} /$ displaced access waveguide $-d=375 \mathrm{~nm}$ ). An interference fringe can be observed in the spectrum for the displaced waveguide excitation, while an almost flat spectrum is obtained when the single mode waveguide is centered since only the even mode is excited. Finally, note that the number of spectral dips available can be significantly increased by simply increasing the length of the SWG bimodal sensor since higher phase shifts are then reached for the same range of wavelengths. 


\section{Spectral sensing response}

The transmission spectrum of the SWG bimodal sensor has been obtained using FDTD simulations when considering different RI claddings, in order to study the sensing characteristics of the final device. As it was previously seen, bulk sensitivity depends on wavelength since the phase shift between the SWG modes shows a non-linear behavior and it reaches an almost zero slope for higher wavelengths within the bimodal bandwidth. Therefore, an accurate design of the sensor is key to obtain optimum spectral dips at critical sensitivity wavelengths to take maximum advantage from the sensor. Making the sensor longer produces more spectral features in the bimodal region, easing the design of high sensitivity fringes. However, when the cladding RI changes, so does the fringe wavelength and consequently its sensing performance.

Figure 6(a) shows the sensor behavior of the higher wavelength spectral feature appearing in the spectrum for two different duty cycles (see Fig. 6(b)). The SWG structures have been designed in order to obtain that spectral feature around $1665 \mathrm{~nm}$ with the purpose of being able to compare the FDTD results with those previously obtained using MPB. In order to locate the spectral feature at that wavelength, we have simply properly selected the number of transversal elements considered in the SWG bimodal structure ( $N$ of 120 and 220 for duty cycles of $50 \%$ and $60 \%$, respectively). Sensitivities of $1070.1 \mathrm{~nm} / \mathrm{RIU}$ and $1375.5 \mathrm{~nm} / \mathrm{RIU}$ have been obtained for duty cycles of $50 \%$ and $60 \%$, respectively. Additionally, Fig. 6(c) depicts the $x$-component of the electric field distribution in FDTD at operating wavelengths corresponding to constructive and destructive interferences.

Moreover, the use of two significantly different lengths also allows us to demonstrate that the spectral shift sensitivity is independent of the sensor length, as well as to confirm that a higher number of spectral fringes is obtained for longer structures (see Fig. 6(b)). It should also be noted that deeper dips are achieved for lower wavelengths, indicating that both SWG modes are almost equally excited, while that modal excitation is not as balanced for higher wavelengths and shallower dips are obtained. Additionally, spectral dips are narrower and more grouped for lower wavelengths as a consequence of the dispersion properties of the odd mode at this region. These two factors make that higher quality factors are achieved for the spectral dips located at lower wavelengths. Therefore, although the spectral sensitivity does not directly depend on the coupling ratio between the two modes and on the number of elements $N$, these are two important parameters to optimize in future experimental developments, since an increase of the quality factor of the spectral dips will allow enhancing the limit of detection of the sensor. On the other hand, we have also to consider that an increase in the number of elements $N$ will be translated into higher propagation losses that could negatively affect the sensor 
performance. However, as it has been previously explained, the dispersive behavior of the modes propagating in the SWG structure allows to obtain a very high sensitivity without the need of very long lengths, what will also contribute to reducing the insertion losses of the sensor.

Finally, a comparative study between the results obtained in MPB and those from the FDTD simulations is presented in Fig. 7. As it can be observed, both approaches match perfectly: the theoretical semi-analytic study of the sensitivity using Eq. (3) with the dispersion relations calculated using MPB and the shift of the spectral fringes measured when making a FDTD analysis of the complete structure.

(a)

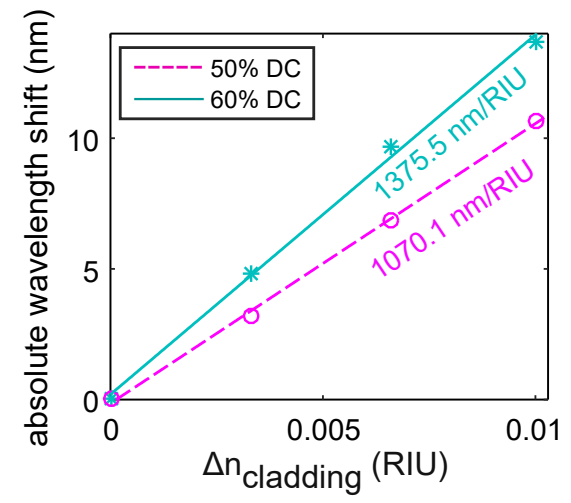

(c)

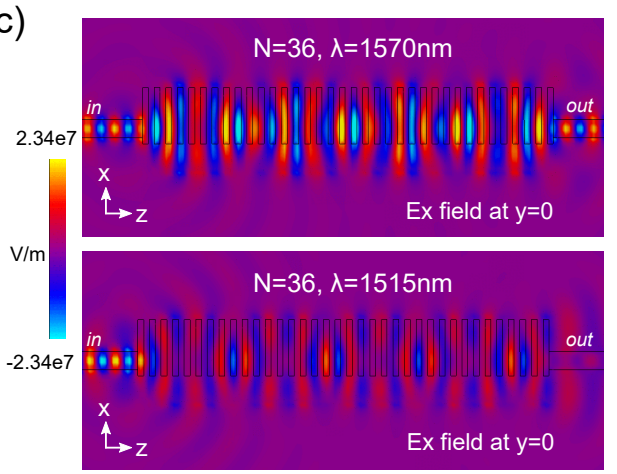

(b)
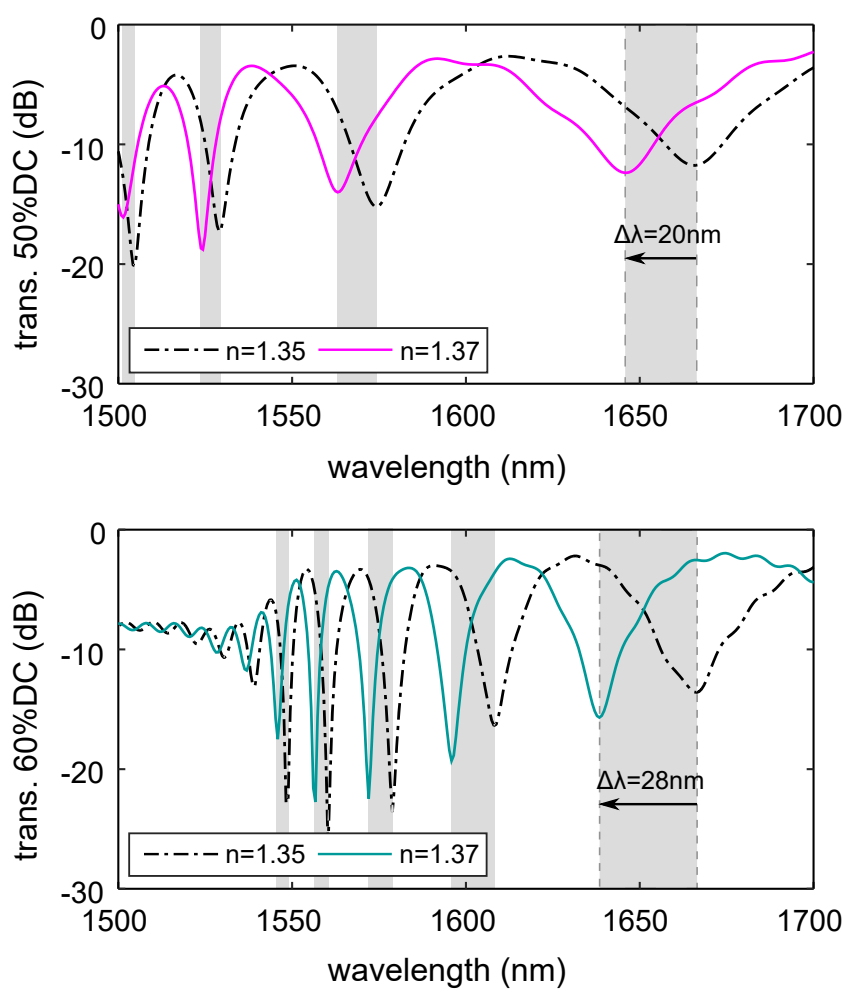

Fig. A.6. (a) Spectral shift as a function of cladding RI variations for $50 \%$ and $60 \%$ duty cycles with $N=120$ and $N=220$ periods, respectively. Design parameters are $\Lambda=290 \mathrm{~nm}$, $w=1400 \mathrm{~nm}, h=220 \mathrm{~nm}$ and $d=375 \mathrm{~nm}$ with a silica lower cladding and under an aqueous environment of 1.36 RIU. (b) Transmission spectra for $50 \%$ duty cycle (top) and $60 \%$ duty cycle (bottom) at different RI scenarios. The shaded areas represent the spectral shift of the interference fringes for a cladding RI increment from 1.35 RIU to 1.37 RIU. (c) $x$-component of the electric field at $y=0$ slice, for $N=36$ periods and $50 \%$ duty cycle. The upper contour map represents maximum transmission for a wavelength exhibiting constructive interference. The lower contour map depicts minimum transmission for a wavelength where a spectral dip is located (i.e., destructive interference). Note that for this number of SWG elements $N$, spectral dips are located at different wavelengths regarding the previous spectrum. 


\subsubsection{Conclusions}

A new sensor concept including SWG structures and single-channel bimodal interferometers has been presented. The combination of these two concepts allows significantly increasing the sensitivity of the device while keeping a compact size. The theoretical background of the SWG bimodal behavior has been studied, as well as its influence in creating critical sensitivity regions in spectral based sensors. A strong dependence with the duty cycle has been determined, since it influences the evolution of the phase shift as a function of wavelength, and thus, the sensitivity. As a result, ultrahigh bulk sensitivity values that can go even above $1300 \mathrm{~nm} / \mathrm{RIU}$ have been obtained both semi-analytically using the dispersion relations of the propagated modes and by means of FDTD simulations. These sensitivity values improve by a factor of 2.5 those reported for SWG RRs, by a factor of almost 6 those reported for conventional RRs and are similar to those reported for other more complex configurations as for the case of slot RRs. Additionally, surface sensitivity has also been studied, obtaining values of up to $6.138 \mathrm{~nm} / \mathrm{nm}$ for $60 \mathrm{~nm}$-thick layers, which are, to the best of our knowledge, the highest reported in the literature for integrated photonic sensors on silicon. Furthermore, the SWG bimodal interferometric sensor also presents outstanding surface sensitivity values

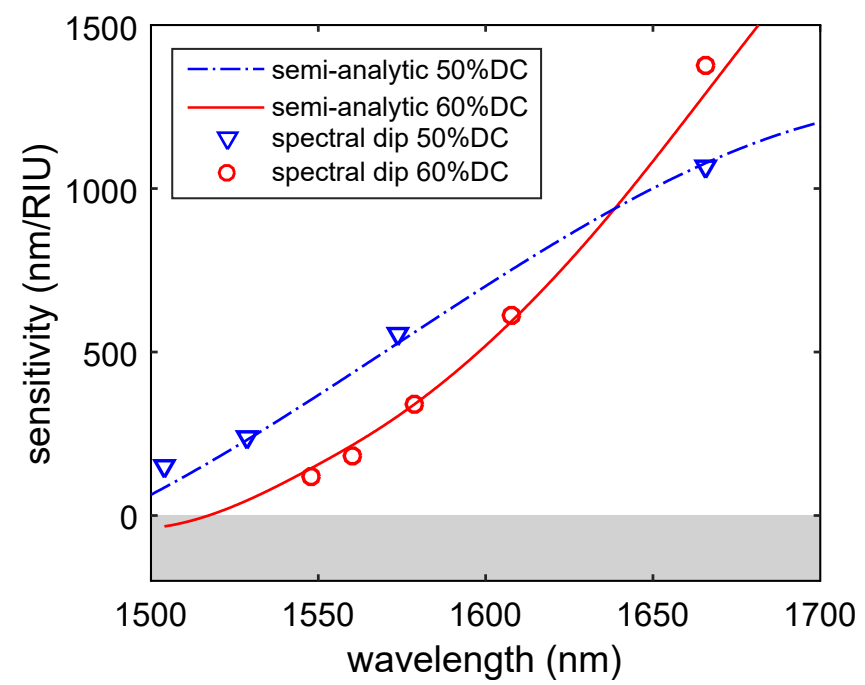

Fig. A.7. Comparison between the sensitivity results obtained in MPB and CST for the SWG sensor having a duty cycle of $50 \%$ and $60 \%$. Semi-analytical curves (solid and dashed lines) represent the theoretical bulk sensitivity as a function of wavelength obtained using MPB and Eq. (3). Diamonds and circle markers show the sensitivity of certain spectral fringes in the spectrum obtained from the FDTD simulations. The parameters of the SWG sensor are $N=120$ for 50 $\%$ duty cycle, $N=220$ for $60 \%$ duty cycle, $\Lambda=290 \mathrm{~nm}, w=1400 \mathrm{~nm}$ and $h=220 \mathrm{~nm}$ in silica lower cladding $(n=1.44)$ and under an aqueous environment of $1.36 \mathrm{RIU}$. The gray shaded area represents the region where the sign of the spectral sensitivity changes. 
of more than $2 \mathrm{~nm} / \mathrm{nm}$ for thicknesses of few nanometers, similarly to the best results in SWG RRs.

\section{Acknowledgements}

European Commission through the Horizon 2020 Programme (PHC-634013 PHOCNOSIS project).

\subsubsection{References}

1. S. M. Rytov, "Electromagnetic properties of a finely stratified medium," JETP, Sov. Phys. 2(3), 466-475 (1956).

2. J. Topol'ančik, P. Bhattacharya, J. Sabarinathan, and P. C. Yu, "Fluid detection with photonic crystal-based multichannel waveguides," Appl. Phys. Lett. 82(8), 1143-1145 (2003).

3. J. D. Joannopoulos, P. R. Villeneuve, and S. Fan, "Photonic crystals: Putting a new twist on light," Nature 386(6621), 143-149 (1997).

4. M. Soljačić, S. G. Johnson, S. Fan, M. Ibanescu, E. Ippen, and J. D. Joannopoulos, "Photonic-crystal slow-light enhancement of nonlinear phase sensitivity," J. Opt. Soc. Am. B 19(9), 2052-2059 (2002)

5. M. L. Povinelli, S. G. Johnson, and J. D. Joannopoulos, "Slow-light, band-edge waveguides for tunable time delays," Opt. Express 13(18), 7145-7159 (2005).

6. E. Chow, A. Grot, L. W. Mirkarimi, M. Sigalas, and G. Girolami, "Ultracompact biochemical sensor built with two-dimensional photonic crystal microcavity," Opt. Lett. 29(10), 1093-1095 (2004).

7. N. Skivesen, A. Têtu, M. Kristensen, J. Kjems, L. H. Frandsen, and P. I. Borel, "Photonic-crystal waveguide biosensor," Opt. Express 15(6), 3169-3176 (2007).

8. J. G. Castelló, V. Toccafondo, P. Pérez-Millán, N. S. Losilla, J. L. Cruz, M. V. Andrés, and J. García-Rupérez, "Real-time and low-cost sensing technique based on photonic bandgap structures," Opt. Lett. 36(14), 2707-2709 (2011).

9. P. Cheben, R. Halir, J. H. Schmid, H. A. Atwater, and D. R. Smith, "Subwavelength integrated photonics," Nature 560(7720), 565-572 (2018).

10. R. Halir, P. Cheben, J. M. Luque-González, J. D. Sarmiento-Merenguel, J. H. Schmid, G. Wangüemert-Pérez, D. X. Xu, S. Wang, A. Ortega-Moñux, and Í. Molina-Fernández, 
"Ultra-broadband nanophotonic beamsplitter using an anisotropic sub-wavelength metamaterial," Laser Photonics Rev. 10(6), 1039-1046 (2016).

11. D. Benedikovic, M. Berciano, C. Alonso-Ramos, X. Le Roux, E. Cassan, D. Marris-Morini, and L. Vivien, "Dispersion control of silicon nanophotonic waveguides using sub-wavelength grating metamaterials in near- and mid-IR wavelengths," Opt. Express 25(16), 19468-19478 (2017).

12. J. M. Luque-González, A. Herrero-Bermello, A. Ortega-Moñux, Í. Molina-Fernández, A. V. Velasco, P. Cheben, J. H. Schmid, S. Wang, and R. Halir, "Tilted subwavelength gratings: controlling anisotropy in metamaterial nanophotonic waveguides," Opt. Lett. 43(19), 4691-4694 (2018).

13. J. Flueckiger, S. Schmidt, V. Donzella, A. Sherwali, D. M. Ratner, L. Chrostowski, and K. C. Cheung, "Sub-wavelength grating for enhanced ring resonator biosensor," Opt. Express 24(14), 15672-15686 (2016).

14. J. Gonzalo Wangüemert-Pérez, P. Cheben, A. Ortega-Moñux, C. Alonso-Ramos, D. Pérez-Galacho, R. Halir, I. Molina-Fernández, D.-X. Xu, and J. H. Schmid, "Evanescent field waveguide sensing with subwavelength grating structures in silicon-on-insulator," Opt. Lett. 39(15), 4442-4445 (2014).

15. J. G. Wangüemert-Pérez, A. Hadij-ElHouati, A. Sánchez-Postigo, J. Leuermann, D. X. Xu, P. Cheben, A. Ortega-Moñux, R. Halir, and Í. Molina-Fernández, "Subwavelength structures for silicon photonics biosensing," Opt. Laser Technol. 109, 437-448 (2019).

16. P. Kozma, F. Kehl, E. Ehrentreich-Förster, C. Stamm, and F. F. Bier, "Integrated planar optical waveguide interferometer biosensors: A comparative review," Biosens. Bioelectron. 58, 287-307 (2014).

17. Q. Liu, X. Tu, K. W. Kim, J. S. Kee, Y. Shin, K. Han, Y. J. Yoon, G. Q. Lo, and M. K. Park, "Highly sensitive Mach-Zehnder interferometer biosensor based on silicon nitride slot waveguide," Sensors Actuators, B Chem. 188, 681-688 (2013).

18. D. Sarkar, N. S. K. Gunda, I. Jamal, and S. K. Mitra, "Optical biosensors with an integrated Mach-Zehnder Interferometer for detection of Listeria monocytogenes," Biomed. Microdevices 16(4), 509-520 (2014).

19. R. Levy and S. Ruschin, "Critical sensitivity in hetero-modal interferometric sensor using spectral interrogation.," Opt. Express 16(25), 20516-20521 (2008).

20. R. Levy, S. Ruschin, and D. Goldring, "Critical sensitivity effect in an interferometer sensor.," Opt. Lett. 34(19), 3023-3025 (2009). 
21. R. Levy and S. Ruschin, "Design of a single-channel modal interferometer waveguide sensor," IEEE Sens. J. 9(2), 146-153 (2009).

22. K. E. Zinoviev, A. B. González-Guerrero, C. Domínguez, and L. M. Lechuga, "Integrated bimodal waveguide interferometric biosensor for label-free analysis," J. Light. Technol. 29(13), 1926-1930 (2011).

23. D. Duval, A. B. González-Guerrero, S. Dante, J. Osmond, R. Monge, L. J. Fernández, K. E. Zinoviev, C. Domínguez, and L. M. Lechuga, "Nanophotonic lab-on-a-chip platforms including novel bimodal interferometers, microfluidics and grating couplers," Lab Chip 12(11), 1987-1994 (2012).

24. C. S. Huertas, D. Fariña, and L. M. Lechuga, "Direct and label-free quantification of micro-RNA-181a at attomolar level in complex media using a nanophotonic biosensor," ACS Sensors 1(6), 748-756 (2016).

25. C. S. Huertas, S. Domínguez-Zotes, and L. M. Lechuga, "Analysis of alternative splicing events for cancer diagnosis using a multiplexing nanophotonic biosensor," Sci. Rep. 7, 1-8 (2017).

26. P. Lalanne and M. Hutley, "Artificial media optical properties - subwavelength scale," Encycl. Opt. Eng. 1, 62-71 (2003).

27. P. J. Bock, P. Cheben, J. H. Schmid, J. Lapointe, A. Delâge, S. Janz, G. C. Aers, D.-X. Xu, A. Densmore, and T. J. Hall, "Subwavelength grating periodic structures in silicon-on-insulator: a new type of microphotonic waveguide," Opt. Express 18(19), 20251-20262 (2010).

28. K. De Vos, I. Bartolozzi, E. Schacht, P. Bienstman, and R. Baets, "Silicon-on-Insulator microring resonator for sensitive and label-free biosensing," 15(12), 7610-7615 (2007).

29. L. Chrostowski, S. Grist, J. Flueckiger, W. Shi, X. Wang, E. Ouellet, H. Yun, M. Webb, B. Nie, Z. Liang, K. C. Cheung, S. A. Schmidt, D. M. Ratner, and N. A. F. Jaeger, "Silicon photonic resonator sensors and devices," Proc. SPIE 8236, 823620 1-16 (2012).

30. S. Johnson and J. Joannopoulos, "Block-iterative frequency-domain methods for Maxwell's equations in a planewave basis," Opt. Express 8(3), 173-190 (2001).

31. E. Luan, H. Yun, L. Laplatine, K. Cheung, Y. Dattner, D. Ratner, J. Flückiger, and L. Chrostowski, "Sub-wavelength multi-box waveguide-based label-free sensors," Integr. Opt. Devices, Mater. Technol. XXII 10535, 105350H (2018).

32. W. Zhang, S. Serna, X. Le Roux, L. Vivien, and E. Cassan, "Highly sensitive refractive index sensing by fast detuning the critical coupling condition of slot waveguide ring resonators," Opt. Lett. 41(3), 532-535 (2016). 
33. A. F. Gavela, D. G. García, J. C. Ramirez, and L. M. Lechuga, "Last advances in silicon-based optical biosensors," Sensors (Basel) 16(3), 285 (2016).

34. E. Luan, H. Yun, L. Laplatine, Y. Dattner, D. M. Ratner, K. C. Cheung, and L. Chrostowski, "Enhanced sensitivity of subwavelength multibox waveguide microring resonator label-free biosensors," IEEE J. Sel. Top. Quantum Electron. 25(3), (2019). 


\subsection{Paper B:}

Optics Letters 44, 4702-4705 (2019)

\section{Experimental study of subwavelength grating bimodal waveguides as ultrasensitive interferometric sensors}

Luis Torrijos-Morán, Amadeu Griol and Jaime García-Rupérez 



\title{
Experimental study of subwavelength grating bimodal waveguides as ultrasensitive interferometric sensors
}

\author{
Luis Torrijos-Morán, Amadeu Griol and Jaime García-Rupérez \\ Nanophotonics Technology Center, Universitat Politècnica de València, \\ Camino de Vera s/n, 46022 Valencia, Spain
}

\begin{abstract}
Over the recent years, subwavelength grating (SWG) structures have increasingly attracted attention in the area of evanescent-field photonic sensors. In this Letter, for the first time to the best of our knowledge, we demonstrate experimentally the real-time refractive index (RI) sensing using the SWG bimodal interferometric structures. Two different configurations are considered to compare the effect of the nonlinear phase shift, obtained between the two first transverse electromagnetic propagating modes, in the measured bulk sensitivity. Very high experimental values up to $2270 \mathrm{~nm} / \mathrm{RIU}$ are reached, which perfectly match the numerical simulations and significantly enhance other existing SWG and spectral-based sensors. By measuring the spectral shift, the obtained experimental sensitivity does not depend on the sensor length. As a result, a highly sensitive and compact single-channel interferometer is experimentally validated for refractive index sensing, thus opening new paths in the field of optical integrated sensors.
\end{abstract}

\subsubsection{Introduction}

A periodic dielectric configuration having a lattice constant smaller than the wavelength of light can be considered a homogeneous anisotropic material in which the electromagnetic wave propagation is feasible [1]. This is the case of the so-called subwavelength gratings (SWGs), first described in [2-4] as an alternative type of silicon waveguides and later on extended to all kind of integrated photonic applications $[5,6]$. More recently, SWG structures have been reported in the literature for refractive index (RI) sensing [7], providing very high bulk and surface sensitivities due to a stronger light-matter interaction with the surrounding media than for conventional waveguides. As a consequence, several sensor configurations such as ring resonators have been redesigned using SWG structures [8-11], exhibiting a superior performance in terms of both the sensitivity and limit of detection. Moreover, SWG also offer additional dispersive properties [12] that can be exploited to develop, for instance, broadband directional couplers [13], beam splitters [14], polarization controllers, [15] and densely integrated 
waveguides [16]. Within this context, we proposed in a previous work the use of a bimodal SWG waveguide as a spectral-interrogationbased sensor [17], where high-sensitivity values were theoretically obtained as a result of the inherent dispersive behavior of SWG structures. On the other hand, similar single-channel interferometers in homogeneous structures have been extensively studied and validated for biosensing purposes [18-20]. In these cases, the sensing performance has been determined by measuring the phase shift, which scales directly with the sensor length, and with the main drawback that large dimensions are required to reach high sensitivities.

In this Letter, we experimentally demonstrate for the first time, to the best of our knowledge, the use of SWG bimodal waveguides as high-performance single-channel interferometric sensors. The underlying idea is to take advantage of the SWG dispersion properties to enhance the spectral shift of a certain interference dip in the spectra. As a result, we show an experimental bulk sensitivity of $2270 \mathrm{~nm} / \mathrm{RIU}$ for a compact SWG bimodal device being only $\sim 125 \mu \mathrm{m}$ long.

\subsubsection{Results}

The sketch of the proposed configuration is depicted in Fig. 1(a), where a single transverse electromagnetic (TE) mode waveguide, acting as the input port, excites the first two TE modes of the bimodal SWG waveguide. Similarly, these two modes will contribute to the excitation of the fundamental mode of the output single-mode waveguide. At this point, the transferred power will depend on the phase shift between the propagating modes of the SWG structure, creating an interference pattern in the transmission spectrum. By tracking the position of a certain spectral dip in the spectrum, caused by a destructive interference between both modes, we can determine the sensitivity of the sensor under different bulk RI variations. In this kind of spectral-interrogation-based sensors, bulk sensitivity can be mathematically expressed as follows [17]:

$$
S_{b}=\frac{\delta \lambda_{f}}{\delta n_{c}}=\frac{\delta \phi / \delta n_{c}}{\delta \phi / \delta \lambda_{f}}
$$

where $\delta \lambda_{f}$ is the differential shift of the spectral dip in $\mathrm{nm}, \delta n_{c}$ is the differential RI change of the cladding in refractive index units (RIU), and $\delta \phi$ is the differential phase shift in radians between the two interfering modes. According to Eq. (1), low slopes of the phase shift as a function of wavelength will increase the the periodic SWG structures created on silicon ( $n=3.47)$ over a silica substrate $(n=1.44)$ were computed using the MIT Photonics Bands (MPB) free software [see Fig. 1(b)]. The design dimensions were the following: SWG bimodal waveguide width $w_{\text {swg }}=1400 \mathrm{~nm}$, height $h=220 \mathrm{~nm}$, lattice period $\Lambda_{1}=$ $260 \mathrm{~nm}$, and transversal elements width $w_{i}=160 \mathrm{~nm}$. In the beginning of the bimodal 
region, at approximately $1500 \mathrm{~nm}$, the even mode is more dispersive than the odd one. In contrast, for higher wavelengths, the even mode becomes less dispersive than the odd mode, producing lower slopes of the phase shift as a function of wavelength, as shown in Fig. 1(c). This fact will provoke high wavelength shifts for those spectral features located at the low-slope wavelength regions. By using different SWG periodicity values, we can tune where this effect occurs and study its influence in the shift of the spectral dips. In Fig. 1(c), we show the evolution of the phase shift as a function of wavelength for $\Lambda_{1}=260 \mathrm{~nm}, w_{i}=$ $160 \mathrm{~nm}$, and a secondary design of $\Lambda_{2}=280 \mathrm{~nm}$ and $w_{i}=180 \mathrm{~nm}$. At higher wavelengths, the slope of both configurations decreases, which will be translated into high spectral shifts of the dips, as indicated by Eq. (1). Nonetheless, for standard operating wavelengths at approximately $1550 \mathrm{~nm}$, this effect will be theoretically higher for the SWG structure of $\Lambda_{1}=260 \mathrm{~nm}$, since we are working in a flatter phase shift region than for the $\Lambda_{2}=280 \mathrm{~nm}$ configuration.

For the experimental demonstration, the sensor was accessed in and out with a single-mode waveguide of width $w_{s}=450 \mathrm{~nm}$. Due to symmetry conditions of the modes field distribution [see Fig. 1(d)], the access single-mode waveguides must be displaced a certain distance " $d$ " with respect to the $x=0$ point to excite the odd parity mode in the SWG region [see Fig. 1(e)]. A displaced distance $d=350 \mathrm{~nm}$ was used for an optimal excitation of both SWG modes.

These designed SWG structures were fabricated on a silicon-on-insulator (SOI) wafer with an upper silicon layer thickness of $220 \mathrm{~nm}$ and a buried silica layer of $2 \mu \mathrm{m}$. Electron beam lithography, with an acceleration voltage of $30 \mathrm{KeV}$ and an aperture size of $30 \mu \mathrm{m}$, was used in the exposure process on an HSQ negative resist, and inductively coupled plasma etching of the top silicon layer was employed to transfer the resist patterns into the SOI wafer. Scanning electron microscope (SEM) images of the fabricated SWG sensors are depicted in Figs. 1(d) and 1(e).

The optical characterization of the fabricated sensors was carried out using a coherent TE polarized light from a tunable laser (Keyshight 81980 ) vertically coupled into the chip using grating couplers. Light collected at the output is measured using an optical power meter (Keyshight 81636B) synchronized with the laser sweep. A LabVIEW application was programed to continuously save the spectrum data, and thus being able to perform time evolution experiments.

First results of the transmission spectra are shown in Fig. 2(a) for a SWG structure of $N=480$ elements, normalized with respect to a uniform reference waveguide in $\mathrm{dB}$ units. The sensor was covered with pure deionized water (DIW) and with a dilution of $6 \%$ ethanol (EtOH) volume in DIW, added gradually on the initial pure DIW dilution to obtain the desired concentrations. The dilutions were directly dropped onto the sample 

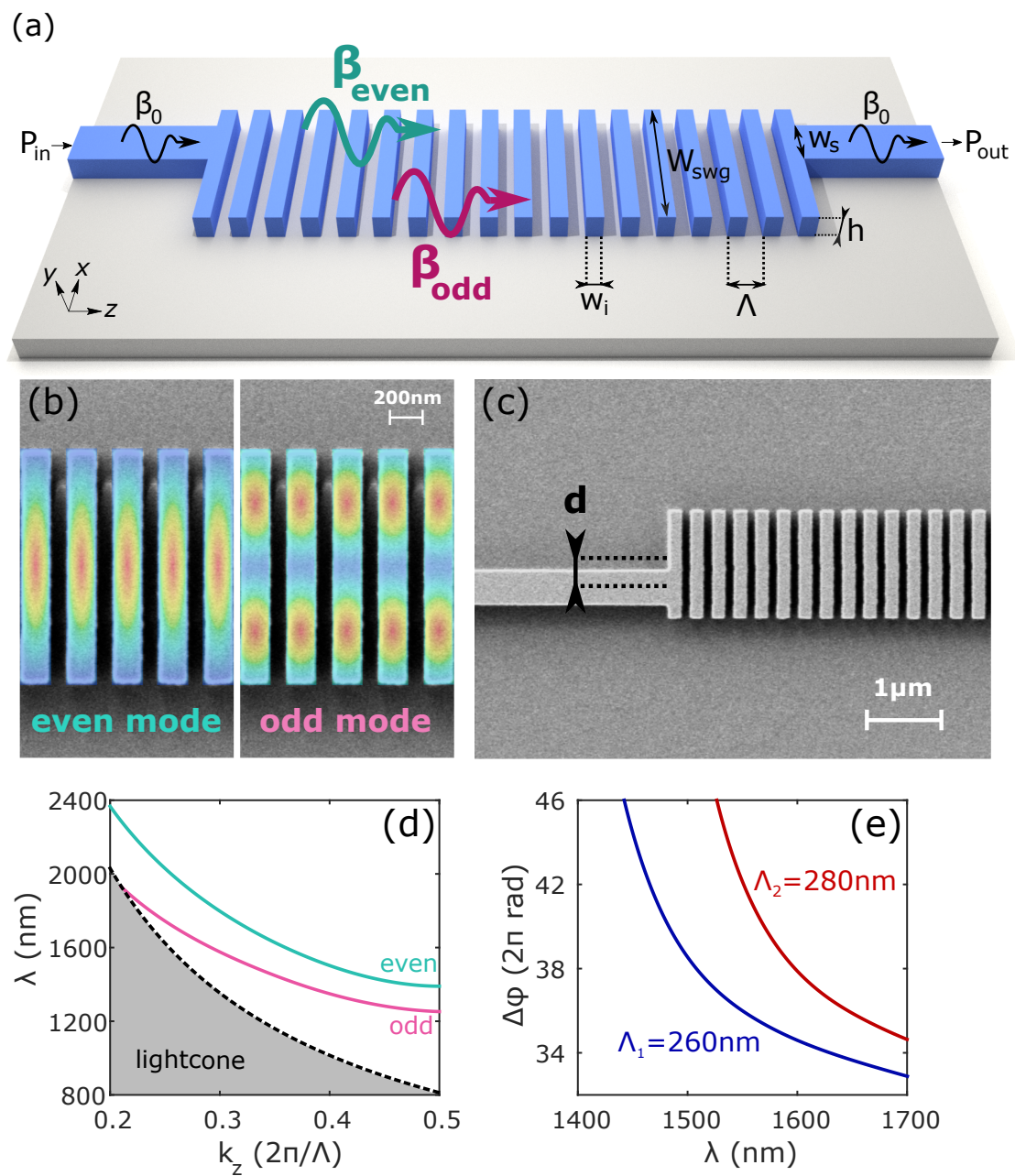

Fig. B.1. (a) Sketch of the proposed bimodal SWG sensor and its design parameters. (b) Dispersion diagram of even and odd modes with $w=1400 \mathrm{~nm}, h=220 \mathrm{~nm}, \Lambda_{1}=260 \mathrm{~nm}$, and $w_{i}=160 \mathrm{~nm}$. (c) Phase shift " $\Delta \phi=L *\left(\beta_{\text {even }}-\beta_{\text {odd }}\right)$ " between the even and odd modes as a function of wavelength for $\Lambda_{1}$ and $\Lambda_{2}=$ configurations, and $N=480$ elements. (d) Electric field energy density of the TE even and odd modes excited in the SWG bimodal region. They are depicted over a SEM image of the fabricated structure. (e) SEM image of the interface between the SWG bimodal waveguide and the single-mode access waveguide, showing an input-output displacement distance of $d=350 \mathrm{~nm}$.

and measured afterwards. According to [22], the RI of these dilutions can be linearly approximated (in mass) for these working conditions $\left(\Lambda=1550 \mathrm{~nm}\right.$ at $25^{\circ} \mathrm{C}$ ), obtaining a value of $n_{D I W}=1.3173$ and $n_{E t O H 6 \%}=1.3205$. The experimental data present a ripple, as a consequence of the Fabry-Perot resonances produced in the SWG discontinuities, that could have a negative effect on the limit of detection since the minimum of the spectral response could not be easily tracked in future biosensing experiments. For that reason, a Lorentzian fitting was applied to the raw data of each spectral dip to better follow the 
evolution of the minimum. As it was theoretically predicted in [17], the spectral dips are shifted toward lower wavelengths when the RI of the dilution increases [see Fig. 2(a)]. In addition, it can be seen how the absolute wavelength shift of the spectra for $\Lambda_{1}=$ $260 \mathrm{~nm}$ is higher than that obtained for the spectra of $\Lambda_{2}=280 \mathrm{~nm}$. This is due to the lower phase shift slope as a function of wavelength obtained for the $\Lambda_{1}$ configuration in comparison with the $\Lambda_{2}$ configuration, and for our experimental wavelength range, as previously described. In addition, note that the spectral shift in both configurations is lower as we decrease the wavelength, which indicates a high dependence of the sensitivity with the dip spectral position.

Conversely, a greater number of spectral dips is experimentally observed in Fig. 2(a) for the configuration with $\Lambda_{2}=280 \mathrm{~nm}$ compared to the other with $\Lambda_{1}=260 \mathrm{~nm}$, in the same range of wavelengths. This is explained by the higher phase shift slope obtained for the $\Lambda_{2}$ configuration that produces more destructive interferences than the $\Lambda_{1}$ configuration in the same bandwidth. As a consequence, the spectral dips will be narrower for the $\Lambda_{2}$ configuration and thus easier to track in future sensing experiments. More specifically, for the $\Lambda_{2}=280 \mathrm{~nm}$ configuration, the full width at half maximum

(a)

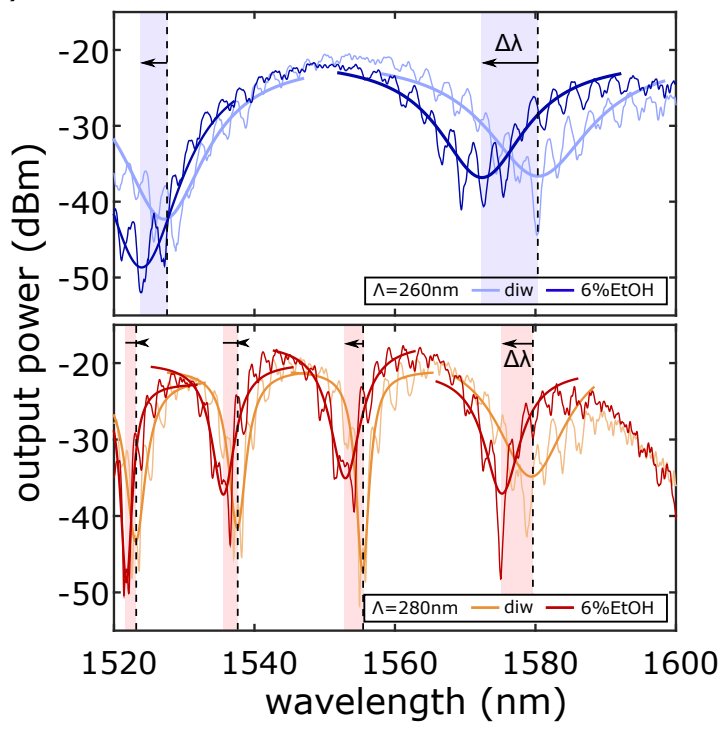

(b)

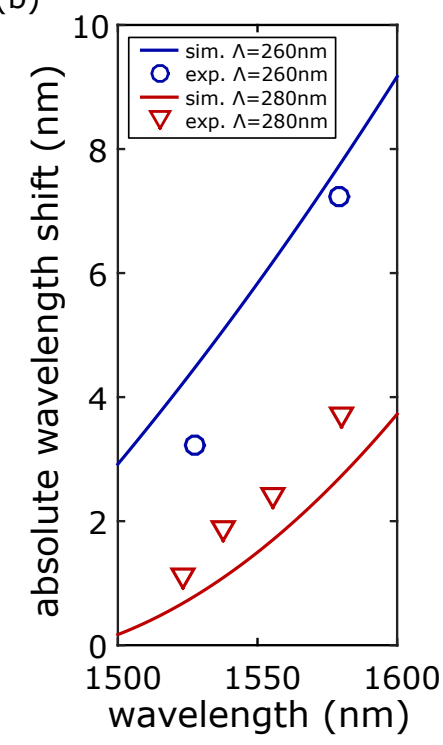

Fig. B.2. (a) Experimental normalized transmission spectra with respect to a reference waveguide, obtained for a bimodal SWG sensor of $N=480$ elements, $\Lambda_{1}=260 \mathrm{~nm}$, and $w_{i}=160 \mathrm{~nm}$ (upper blue graph), and $\Lambda_{2}=280 \mathrm{~nm}, w_{i}=180 \mathrm{~nm}$ (lower red graph). A Lorentzian fitting is also depicted over the spectral dips to ease the determination of the minimum location. (b) Experimental comparison between the numerical simulations and the absolute wavelength shifts of each spectral dip for a refractive index change of $3.2 \times 10^{-3} \mathrm{RIU}$ as a function of wavelength, and for the periods of $\Lambda_{1}=260 \mathrm{~nm}$ and $\Lambda_{2}=280 \mathrm{~nm}$. The lines show the numerical simulations for a continuous range of wavelengths, and the markers show the experimental values of each spectral dip located at a certain wavelength. 
(FWHM) of the most sensitive spectral dip located at $1580 \mathrm{~nm}$ is twice that for the lowest dip at $1523 \mathrm{~nm}$. Therefore, a trade-off between sensitivity and narrower spectral dips is observed, although the FWHM of the most sensitive dips could be reduced by increasing the number of elements $N$.

To compare the results with simulations, the dispersion relations of both SWG modes were computed under different cladding RI scenarios of pure DIW and $6 \%$ ethanol volume in DIW. The computed absolute wavelength shift of each spectral dip and the experimental results are depicted in Fig. 2(b) for both configurations,showing a good agreement between theory and measurements for a RI increment of $3.2 \times 10^{-3}$ RIU. Moreover, it should be noted again that in Fig. 2(b) the shift is strongly dependent on wavelength because of the nonlinear phase shift behavior of the SWG structure. This fact provokes higher sensitivities for those spectral dips located at higher wavelengths.

Likewise, several time evolution measurements were carried out by covering the SWG sensor with different ethanol dilutions. To this end, real-time spectrum data were collected for $35 \mathrm{~min}$ and subsequently processed to properly track the minimum of each spectral dip over time. Figure 3(a) shows the tracking of the spectral dip located at 1580 $\mathrm{nm}$ for $\Lambda_{1}=260 \mathrm{~nm}$ and $\Lambda_{2}=280 \mathrm{~nm}$ configurations. Ethanol dilutions of $2 \%, 4 \%$, and $6 \%$ in DIW were considered for the experiments, corresponding to a RI of 1.3183, 1.3194, and 1.3205 , respectively. The absolute wavelength shift versus RI variations is depicted in Fig. 3(b) for both configurations. In this graph, we obtain a bulk sensitivity of $2270 \mathrm{~nm} / \mathrm{RIU}$ for the SWG configuration with $\Lambda_{1}=260 \mathrm{~nm}$ and a value of $1253 \mathrm{~nm} / \mathrm{RIU}$ for the SWG with $\Lambda_{2}=280 \mathrm{~nm}$. These results are in good agreement with the numerically simulations previously calculated and consistent with the phase shift slopes presented in Fig. 1(c). Note that there is a period of time that the measurements take to stabilize, and this is due to the RI variations occurring when new ethanol dilutions are added.

In comparison with the literature, SWG spectral-based sensors such as multi-box ring resonators [23] have shown an experimental bulk sensitivity of $580 \mathrm{~nm} / \mathrm{RIU}$, and other configurations like slot ring resonators [24] have been experimentally demonstrated for RI sensing with sensitivities up to $1300 \mathrm{~nm} / \mathrm{RIU}$, as well as slotted photonic crystal sensors [25] with a reported sensitivity of $1538 \mathrm{~nm} /$ RIU. On the other hand, similar bimodal interferometers [26] using homogeneous waveguides have been presented as spectral-based sensors with sensitivity values of $789 \mathrm{~nm} / \mathrm{RIU}$. Therefore, our proposed sensor presents a markedly higher bulk sensitivity than previous examples, while keeping a very low structural complexity, thus confirming the high potential of SWG bimodal waveguides as a promising alternative for sensing applications in CMOS-compatible integrated devices. 
(a)

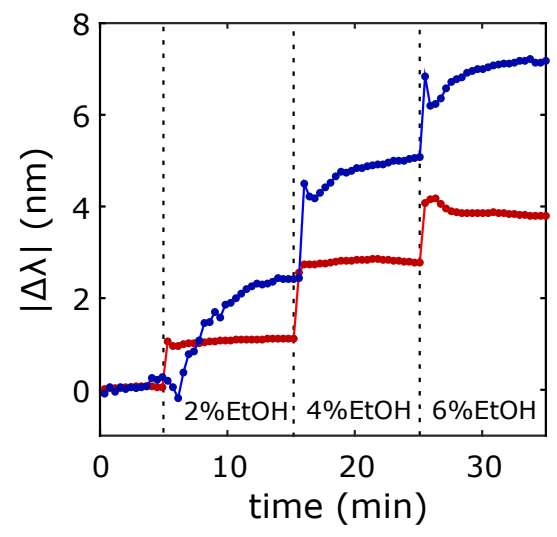

(b)

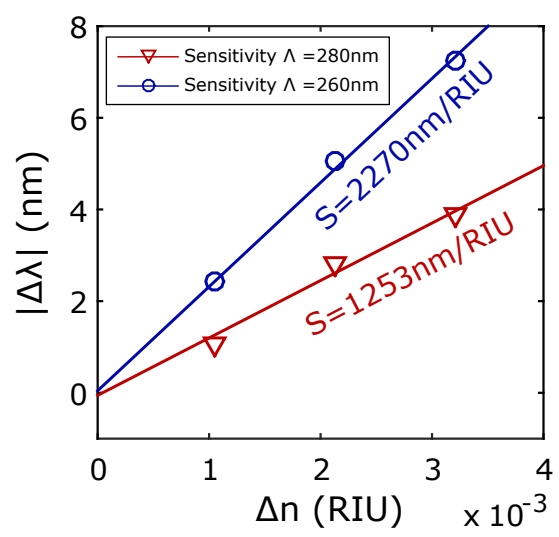

Fig. B.3. (a) Time evolution of the spectral dips located at $1580 \mathrm{~nm}$ with $N=480$ elements for $\Lambda_{1}=260 \mathrm{~nm}, w_{i}=160 \mathrm{~nm}$ (blue curve) and $\Lambda_{2}=280 \mathrm{~nm}, w_{i}=180 \mathrm{~nm}$ (red curve) under three different dilutions of $2 \%, 4 \%$, and $6 \%$ of ethanol volume in DIW. (b) Representation of the absolute wavelength shift of the spectral dips under different RI variations. The linear fitting represents the bulk sensitivity experimentally obtained for both configurations.

To investigate the dependence of the experimental sensitivity with the sensor length, the spectral shift of several dips with SWG configurations of $N=120, N=240, N=360$, and $N=480$ elements for a RI change of $5.39 \times 10^{-3}$ RIU was also measured. The rest of the design parameters are the same used in the previous analysis ( $w_{s}=450 \mathrm{~nm}, w=$ $1400 \mathrm{~nm}, h=220 \mathrm{~nm}, d=350 \mathrm{~nm}, \Lambda_{2}=280 \mathrm{~nm}$, and $w_{i}=180 \mathrm{~nm}$ ). Figure 4(a) depicts numerical simulations for the numerator and the denominator of Eq. (1), varying the number $N$ of SWG elements. In this graph, we can clearly see that the value of the numerator and denominator highly depends on $N$ and wavelength. However, when we apply Eq. (1) and divide both expressions, the theoretical sensitivity obtained remains constant for any value of $N$, although it still depends on wavelength. This theoretical sensitivity curve is shown in Fig. 4(b) as a function of wavelength. The colored bars represent the experimental sensitivity of each spectral dip obtained for different $N$ at a certain wavelength. All the measurements are in a good agreement with the simulations and remain on the line of Eq. (1). These results demonstrate that the bulk sensitivity, measuring the wavelength shift, does not depend on the sensor length, as it occurs in interferometric configurations when measuring the phase shift.

Despite this, as it has been explained before, the quality factor of the spectral dip increases with $N$ since it is more grouped in the spectrum, and narrower spectral features are obtained. As a result, longer SWG structures will facilitate the tracking of those dips and thus enhance the limit of detection of the sensor, although it will increase its footprint, which is not very suitable for integration purposes. Future optimized designs must be made to enhance the sensor limit of detection, and thus compare it to other 
(a)

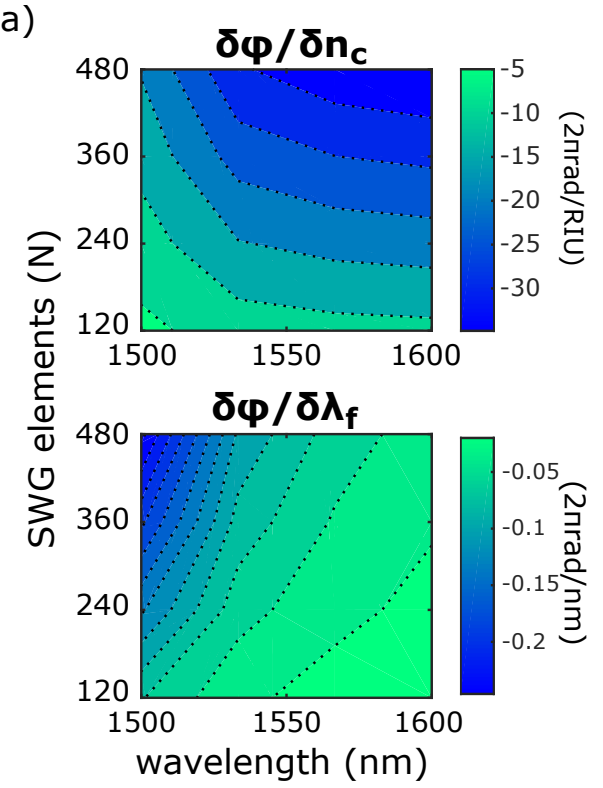

(b)

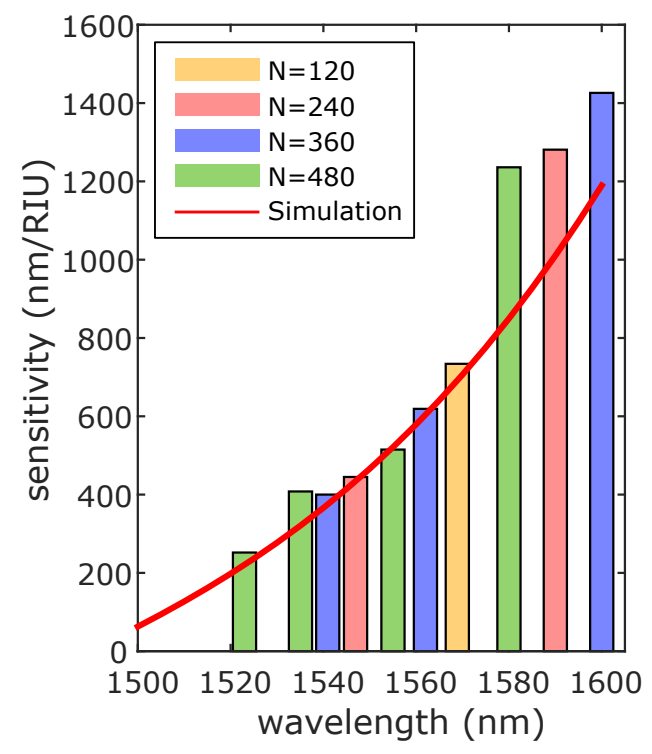

Fig. B.4. (a) Color map representing the numerical derivative of the phase shift with respect to the cladding RI (upper graph) and wavelength (lower graph), for a different number of periods $N$ and wavelengths. (b) Comparison between the simulated sensitivity obtained using Eq. (1) (red curve) and the experimental results considering a different number of periods $N$ (colored bars). The SWG dimensions used for the simulation and experimental measurements are $w=1400 \mathrm{~nm}$, $h=220 \mathrm{~nm}, d=350 \mathrm{~nm}, \Lambda_{2}=280 \mathrm{~nm}$, and $w_{i}=180 \mathrm{~nm}$.

existing devices in biosensing applications [27]. The obtained transmission spectra for a different $N$ were compared to the one recorded with a single-mode waveguide, with the aim of measuring the insertion losses of the sensor. A value around $1 \mathrm{~dB}$ was obtained for any number of elements $N$, which means that losses are mainly due to the coupling between the single-mode access waveguides and the bimodal SWG, and that propagation losses can be considered practically negligible, thus allowing the use of long bimodal SWG sections, if desired.

\subsubsection{Conclusions}

To conclude, in this Letter, we have demonstrated the use of bimodal SWG waveguides for RI sensing and studied their high-performance experimental features. The proposed mechanism relies on the dispersive behavior of SWG structures to critically enhance the wavelength shift of the spectral dips when varying the RI of the cladding. A complete comparison of experimental results and simulations is provided, as well as time evolution measurements to evaluate its bulk sensitivity. Values up to $2270 \mathrm{~nm} / \mathrm{RIU}$ have been obtained for a SWG periodicity and element width of $260 \mathrm{~nm}$ and $160 \mathrm{~nm}$, respectively. To the best of our knowledge, these experimental results are the highest reported in the 
literature for an integrated silicon RI sensor. Furthermore, its scalable properties allow us to design the sensor to work at the desired wavelength by simply changing the lattice period of the structure. Overall, its compact fully etched single-channel design and its outstanding sensitivity place it as a promising alternative for lab-on-a-chip devices in future work.

\section{Funding}

European Commission (PHC-634013 PHOCNOSIS project); Spanish Government (TEC2015- 63838-C3-1-R-OPTONANOSENS project); Universitat Politècnica de València (grant PAID 01-18).

\subsubsection{References}

1. S. M. Rytov, "Electromagnetic properties of a finely stratified medium," JETP, Sov. Phys. 2(3), 466-475 (1956).

2. P. Cheben, D.-X. Xu, S. Janz, and A. Densmore, "Subwavelength waveguide grating for mode conversion and light coupling in integrated optics," Opt. Express 14, 4695 (2006).

3. D.-X. Xu, E. Post, J. Lapointe, P. Cheben, S. Janz, and J. H. Schmid, "Gradient-index antireflective subwavelength structures for planar waveguide facets," Opt. Lett. 32, 1794 (2007).

4. P. J. Bock, P. Cheben, J. H. Schmid, J. Lapointe, A. Delâge, S. Janz, G. C. Aers, D.-X. $\mathrm{Xu}, \mathrm{A}$. Densmore, and T. J. Hall, "Subwavelength grating periodic structures in silicon-on-insulator: a new type of microphotonic waveguide," Opt. Express 18, 20251-20262 (2010).

5. R. Halir, P. J. Bock, P. Cheben, A. Ortega-Moñux, C. Alonso-Ramos, J. H. Schmid, J. Lapointe, D. X. Xu, J. G. Wangüemert-Pérez, Í. Molina-Fernández, and S. Janz, "Waveguide sub-wavelength structures: A review of principles and applications," Laser Photonics Rev. 9, 25-49 (2015).

6. P. Cheben, R. Halir, J. H. Schmid, H. A. Atwater, and D. R. Smith, "Subwavelength integrated photonics," Nature 560, 565-572 (2018).

7. J. Gonzalo Wangüemert-Pérez, P. Cheben, A. Ortega-Moñux, C. Alonso-Ramos, D. Pérez-Galacho, R. Halir, I. Molina-Fernández, D.-X. Xu, and J. H. Schmid, "Evanescent field waveguide sensing with subwavelength grating structures in silicon-on-insulator," Opt. Lett. 39, 4442-4445 (2014). 
8. V. Donzella, A. Sherwali, J. Flueckiger, S. M. Grist, S. T. Fard, and L. Chrostowski, "Design and fabrication of SOI micro-ring resonators based on sub-wavelength grating waveguides," Opt. Express 23, 4791 (2015).

9. J. Flueckiger, S. Schmidt, V. Donzella, A. Sherwali, D. M. Ratner, L. Chrostowski, and K. C. Cheung, "Sub-wavelength grating for enhanced ring resonator biosensor," Opt. Express 24, 15672-15686 (2016).

10. H. Yan, L. Huang, X. Xu, S. Chakravarty, N. Tang, H. Tian, and R. T. Chen, "Unique surface sensing property and enhanced sensitivity in microring resonator biosensors based on subwavelength grating waveguides," Opt. Express 24, 29724 (2016).

11. H. Tian, H. Yan, N. Tang, R. T. Chen, S. Chakravarty, L. Huang, and X. Xu, "Improving the detection limit for on-chip photonic sensors based on subwavelength grating racetrack resonators," Opt. Express 25, 10527 (2017).

12. D. Benedikovic, M. Berciano, C. Alonso-Ramos, X. Le Roux, E. Cassan, D. Marris-Morini, and L. Vivien, "Dispersion control of silicon nanophotonic waveguides using sub-wavelength grating metamaterials in near- and mid-IR wavelengths," Opt. Express 25, 19468-19478 (2017).

13. Y. Wang, Z. Lu, M. Ma, H. Yun, F. Zhang, N. A. F. Jaeger, and L. Chrostowski, "Compact Broadband Directional Couplers Using Subwavelength Gratings," IEEE Photonics J. 8, 1-8 (2016).

14. R. Halir, P. Cheben, J. M. Luque-González, J. D. Sarmiento-Merenguel, J. H. Schmid, G. Wangüemert-Pérez, D. X. Xu, S. Wang, A. Ortega-Moñux, and Í. Molina-Fernández, "Ultra-broadband nanophotonic beamsplitter using an anisotropic sub-wavelength metamaterial," Laser Photonics Rev. 10, 1039-1046 (2016).

15. J. M. Luque-González, A. Herrero-Bermello, A. Ortega-Moñux, Í. Molina-Fernández, A. V. Velasco, P. Cheben, J. H. Schmid, S. Wang, and R. Halir, "Tilted subwavelength gratings: controlling anisotropy in metamaterial nanophotonic waveguides," Opt. Lett. 43, 4691-4694 (2018).

16. S. Jahani, S. Kim, J. Atkinson, J. C. Wirth, F. Kalhor, A. Al Noman, W. D. Newman, P. Shekhar, K. Han, V. Van, R. G. Decorby, L. Chrostowski, M. Qi, and Z. Jacob, "Controlling evanescent waves using silicon photonic all-dielectric metamaterials for dense integration," Nat. Commun. 9, (2018).

17. L. Torrijos-Morán and J. García-Rupérez, "Single-channel bimodal interferometric sensor using subwavelength structures," Opt. Express 27, 8168-8179 (2019). 
18. R. Levy and S. Ruschin, "Design of a single-channel modal interferometer waveguide sensor," IEEE Sens. J. 9, 146-153 (2009).

19. K. E. Zinoviev, A. B. González-Guerrero, C. Domínguez, and L. M. Lechuga, "Integrated bimodal waveguide interferometric biosensor for label-free analysis," J. Light. Technol. 29, 1926-1930 (2011).

20. P. Kozma, F. Kehl, E. Ehrentreich-Förster, C. Stamm, and F. F. Bier, "Integrated planar optical waveguide interferometer biosensors: A comparative review," Biosens. Bioelectron. 58, 287-307 (2014).

21. R. Levy and S. Ruschin, "Critical sensitivity in hetero-modal interferometric sensor using spectral interrogation.," Opt. Express 16, 20516-20521 (2008).

22. J. García-Rupérez, V. Toccafondo, M. J. Bañuls, J. G. Castelló, A. Griol, S. Peransi-Llopis, and Á. Maquieira, "Label-free antibody detection using band edge fringes in SOI planar photonic crystal waveguides in the slow-light regime.," Opt. Express 18, 24276-24286 (2010).

23. E. Luan, H. Yun, L. Laplatine, K. Cheung, Y. Dattner, D. Ratner, J. Flückiger, and L. Chrostowski, "Sub-wavelength multi-box waveguide-based label-free sensors," Integr. Opt. Devices, Mater. Technol. XXII 10535, 105350H (2018).

24. W. Zhang, S. Serna, X. Le Roux, L. Vivien, and E. Cassan, "Highly sensitive refractive index sensing by fast detuning the critical coupling condition of slot waveguide ring resonators," Opt. Lett. 41, 532-535 (2016).

25. A. Di Falco, L. O’Faolain, and T. F. Krauss, "Chemical sensing in slotted photonic crystal heterostructure cavities," Appl. Phys. Lett. 94, 92-95 (2009).

26. Y. Liang, M. Zhao, Z. Wu, and G. Morthier, "Bimodal Waveguide Interferometer RI Sensor Fabricated on Low-Cost Polymer Platform," IEEE Photonics J. 11, (2019).

27. Í. Molina-Fernández, J. Leuermann, A. Ortega-Moñux, J. G. Wangüemert-Pérez, and R. Halir, "Fundamental limit of detection of photonic biosensors with coherent phase read-out," Opt. Express 27, 12616 (2019). 



\section{Chapter 4}

\section{Bimodal interferometers in one-dimensional photonic crystals}

Integrated interferometers are fundamental components of current optical circuits. Among others, the leading candidate is the MZI, which has been employed since the end of the last century to develop devices such as modulators and sensors [117-119]. However, the demands of densely integrated chips are significant, and herein conventional MZIs do not offer clear solutions due to their usually large design area [120-122]. To address some of these size limitations, PhCs provide remarkable advantages by building MZI configurations with 2D structures [123-131], or integrating simpler 1D PhCs within the MZI arms [132-134], to develop, for instance, ultra-compact modulators and switches. To this end, 1D PhCs are easier to fabricate and provide a smaller lateral size compared to 2D and 3D structures, which is also desirable for integration purposes [135-142]. Moreover, they preserve most part of PhC benefits, specifically the slow light effects that may be used to reduce the interferometer length [143-147]. Even so, MZI-based configurations require two paths to perform the interferometry, which hinders the integration of multiple devices in a single circuit. On this matter, BiM waveguides provide common path solutions as integrated interferomters with significant applications in biosensing [148153], although long physical paths are still needed.

The following chapter contains original works on the design and experimental verification of one-dimensional photonic crystals for bimodal operation. In these articles, we encompass the benefits from both slow light structures and bimodal waveguides to develop common path interferometers as high-performance photonic devices such as modulators and sensors (see Paper C), as well as optical switches (see Paper D), all of them in extremely compact footprints. In turn, Paper E provides a new design method to optimize multi-parameter photonic crystals to enhance the bimodal behavior. 



\subsection{Paper C:}

Light: Science \& Applications 10, 16 (2021)

\section{Slow light bimodal interferometry in one-dimensional photonic crystal waveguides}

Luis Torrijos-Morán, Amadeu Griol and Jaime García-Rupérez 



\title{
Slow light bimodal interferometry in one-dimensional photonic crystal waveguides
}

\author{
Luis Torrijos-Morán, Amadeu Griol and Jaime García-Rupérez \\ Nanophotonics Technology Center, Universitat Politècnica de València, \\ Camino de Vera s/n, 46022 Valencia, Spain
}

\begin{abstract}
Strongly influenced by the advances in the semiconductor industry, the miniaturization and integration of optical circuits into smaller devices has stimulated considerable research efforts in recent decades. Among other structures, integrated interferometers play a prominent role in the development of photonic devices for on-chip applications ranging from optical communication networks to point-of-care analysis instruments. However, it has been a long-standing challenge to design extremely short interferometer schemes, as long interaction lengths are typically required for a complete modulation transition. Several approaches, including novel materials or sophisticated configurations, have been proposed to overcome some of these size limitations but at the expense of increasing fabrication complexity and cost. Here, we demonstrate for the first time slow light bimodal interferometric behaviour in an integrated single-channel one-dimensional photonic crystal. The proposed structure supports two electromagnetic modes of the same polarization that exhibit a large group velocity difference. Specifically, an over 20 -fold reduction in the higher-order-mode group velocity is experimentally shown on a straightforward all-dielectric bimodal structure, leading to a remarkable optical path reduction compared to other conventional interferometers. Moreover, we experimentally demonstrate the significant performance improvement provided by the proposed bimodal photonic crystal interferometer in the creation of an ultra-compact optical modulator and a highly sensitive photonic sensor.
\end{abstract}

\subsubsection{Introduction}

The slowing down of light was first theoretically described by Hendrik Lorentz more than a century ago, when it was shown that the group velocity can be drastically decreased in the presence of an ultracold atomic vapour [1]. More recently, in 1999, these predictions were experimentally demonstrated, and a light speed of just $17 \mathrm{~m} / \mathrm{s}$ was achieved using an electromagnetically induced transparency quantum phenomenon [2]. This intriguing 
finding captured the interest of the research community and gave rise to significant work aimed at producing slow light in solids at room temperature. Coherent population oscillation processes were introduced years later to solve this issue, and the feasibility of this effect was shown in ruby and alexandrite crystals [3,4]. Likewise, material engineered structures can create artificial optical resonances and produce slow light. This is the case for photonic crystal $(\mathrm{PhC})$ structures that consist of periodic dielectric repetition for any of the three spatial dimensions and where the group velocity of the supported modes is dramatically reduced near the edge of the Brillouin zone [5]. PhCs can also exhibit one or several photonic bandgap (PBG) regions, in which certain frequencies are not allowed to propagate through the structure and where slow light is produced near the edge of the bands defining these PBGs [6]. Additionally, slow light behaviour can be observed for guided modes propagating through linear defects introduced in $\mathrm{PhC}$ structures. For instance, two-dimensional (2D) hole patterned $\mathrm{PhC}$ waveguides were exploited to show the active control of light, and an over 300-fold reduction in the group velocity was achieved on a compact silicon integrated circuit [7]. Slow light also enables us to temporarily store optical signals or provoke a stronger light-matter interaction that enhances optical phase non-linearities [8,9], among other advantages. Nonetheless, PhCs present some limitations regarding the operating bandwidth as well as coupling losses and the tuning of the slow modes [10].

In this context, three-dimensional (3D) and 2D PhCs have been used in subsequent years for the creation of 3D PBGs at near-infrared wavelengths [11], ultra-compact optical switches [12], refractive index (RI) biosensors [13] or near-zero RI materials [14]. However, the combination of these types of PhCs with other integrated structures can be challenging due to their structural complexity, which hinders fabrication processes for mass production [15]. Instead, more straightforward designs based on one-dimensional (1D) periodic waveguides ease the fabrication process while preserving the slow light benefits of phase non-linearities and dispersion tunability [16]. Furthermore, these structures have a smaller lateral size in comparison with 2D PhCs, which reduces the footprint of the device. These were first demonstrated for the propagation of ultrashort pulses near the band edge with large group delays [17] and as short resonators in planar integrated platforms [18]. Quasi-one-dimensional PhCs based on Bragg grating structures were subsequently employed for refractometric sensing [19], and 1D PhC waveguides were reported for integrated tuneable time delay devices [20] and negative group velocity anomalous phenomena [21]. These types of corrugated waveguides were demonstrated with low propagation losses below $1 \mathrm{~dB}$ [22], which had a significant impact years later in the development of several applications, such as mid-infrared slow light engineering waveguides [23] or label-free biosensors [24]. 1D PhC structures have also been extensively used in $\mathrm{PhC}$ enhancement microscopy applications, as in the case of 
enhanced fluorescence to quantify the concentration of a certain analyte in a liquid sample [25].

Since the early 1990s, optical interferometry based on Mach-Zehnder interferometer (MZI)-integrated schemes has been extensively studied for the development of devices such as silicon modulators [26] and biosensors [27]. Basically, in an MZI, light is split into two different optical paths and recombined to create an interference pattern at the output signal. Changes in the real part of the material RI induced, for example, by a temperature change or by an applied electric field produce a relative phase shift with respect to the reference arm. MZI-based systems have since been employed in making compact high-speed low-power consumption silicon modulators [28,29], as well as high-performance integrated devices for biosensing [30]. Nevertheless, in all of the abovementioned approaches, the performance of the MZI scales with the length of the optical paths, which makes it very difficult to design compact interferometers with high operational features. Accordingly, plasmonic interferometers have been introduced in recent years to overcome some of these drawbacks, creating high-speed ultra-compact and low energy consumption modulators $[31,32,33]$. Similarly, nano-slits in a thin metal film have been employed to develop plasmonic MZI on-chip biosensors with very high bulk RI sensitivity [34]. Moreover, other MZI configurations involving novel materials such as graphene, indium tin oxide (ITO) and lithium niobate have been investigated for their broadband and efficient electro-optical responses [35, 36, 37]. Although these interferometers offer prominent breakthroughs in comparison with classic dielectric MZI configurations, the use of new materials makes the fabrication processes complex and adds extra costs and difficulties to the micro-structuring of photonic circuits.

Integrating all-dielectric slow light elements in MZI schemes offers some advantages in terms of footprint reduction for the final device while maintaining a fully silicon-based structure. This idea was initially introduced at the end of the last century, where grating structures were included in the optical paths of an MZI modulator to improve its efficiency by reducing the group velocity of the propagating modes [38]. Later, this concept was extended to 2D PhCs to develop a highly compact asymmetric MZI of only $20 \mu \mathrm{m}$ in length [39], as well as high-speed low-voltage modulators employing polymer-infiltrated materials [40] or embedded PhC cavities based on electro-optic [41] or thermo-optic effects [42] with switching speed limitations [43]. For 1D PhC waveguides, high-speed electro-optic modulators of $500 \mu \mathrm{m}$ length, including a corrugated waveguide in one of the MZI arms, have been proposed for a dense integration level in foreseeable network-on-chip devices [44]. Similar MZI designs, including highly dispersive 1D periodic structures made of embedded circular holes in rectangular waveguides, have also been validated for achieving very short biosensing devices [45]. However, classic MZI-based configurations require additional photonic structures, such as power splitters 
or different optical waveguides, to perform interferometry. In this scenario, bimodal waveguide sensors were proposed to address limitations regarding compactness and ease of fabrication [46]. The underlying concept of these interferometers relies on exciting the first two electromagnetic modes that have the same polarization in an optical waveguide and making them interfere, converting a change in RI into an intensity modulation. Since both modes do not interact equally with an induced RI change, a phase shift is therefore produced between the fundamental mode, acting as a reference, and a higher-order mode, acting as an active mode. The higher-order mode is more sensitive to changes in the RI than the fundamental mode in a conventional MZI sensing arm, leading to a higher accumulated phase shift. This principle has been extensively studied in recent work for biosensing applications in lab-on-a-chip platforms [47]. Other bimodal concepts have also been proposed by our group for ultra-high spectral-based sensitivity in silicon periodic structures in the subwavelength regime $[48,49]$. Nonetheless, in this latter case, the operation principle is different from that of standard bimodal waveguides since both modes present similar dispersion properties and equally interact with the surrounding medium and thus create very large shifts in the spectral interferences.

In this work, we propose a short and single-channel bimodal interferometer enabled by all-dielectric 1D $\mathrm{PhC}$ waveguides working in the slow light regime at telecom wavelengths. We optimize and experimentally demonstrate the periodic structure to support a dispersive higher-order mode (acting as an active mode) with a drastically reduced group velocity in comparison to the fundamental mode (acting as reference). Our design encompasses benefits from PhCs and bimodal-based interferometers in terms of sensitivity and compactness by including slow light elements of straightforward 1D designs. We also experimentally demonstrate the operation of these interferometers when temperature and cladding RI changes are produced, confirming them as promising alternatives for high-efficiency modulators and high-sensitivity RI sensors, respectively, both with extremely reduced footprints.

\subsubsection{Results}

\section{Principle of operation}

The proposed design, which is shown in Fig. 1a, is fully based on a silicon structure surrounded by silica cladding, in which a single-mode input waveguide, supporting the fundamental mode of transverse electric (TE) polarization, transfers its power to the first two TE-like even modes in the bimodal region of the 1D PhC: TE0-like and TE2-like. These two modes propagate through the $1 \mathrm{D} \mathrm{PhC}$ and, after a certain distance, interfere in the abrupt discontinuity with the exit single-mode waveguide and thus contribute to the 
excitation of the fundamental TE mode at the output. Therefore, the transferred power may be expressed as a function of the phase shift accumulated between both modes in the bimodal region. Consequently, as occurs with a conventional MZI, by measuring the interference pattern in the transmission spectra, we obtain information about the phase shift between both modes and how it varies when a change in the RI is induced. In addition, a rectangular taper is placed in the transition between the single-mode waveguide and the bimodal $\mathrm{PhC}$ waveguide for efficient modal excitation in the periodic section. The bimodal interferometric structure is composed of a repetition of a basic unit cell consisting of a transversal element (or corrugation) over a central rectangular waveguide (see the inset of Fig. 1a), thereby creating a 1D PhC in the z-axis propagation direction. The design parameters of the proposed unit cell are a lattice period $a=370$ $\mathrm{nm}$, transversal element width $w_{i}=220 \mathrm{~nm}$, transversal element length $w_{e}=1400 \mathrm{~nm}$, central waveguide width $w=600 \mathrm{~nm}$ and height $h=220 \mathrm{~nm}$, accessed in and out with a single-mode waveguide of width $w_{s}=450 \mathrm{~nm}$.

Figure $1 \mathrm{~b}$ depicts a dispersion diagram of the designed $1 \mathrm{D} \mathrm{PhC}$ in the irreducible Brillouin zone, showing the TE-like bands that will be excited by the fundamental TE mode of the single-mode input waveguide. Only those bands presenting an even parity with respect to the $x=0$ plane are considered, since the odd parity modes will not be excited, as in the case of the first-order mode ( $\mathrm{TE}_{1}$-like), due to symmetry conditions in the interface between the single-mode waveguides and the bimodal waveguide. The first three bands are depicted and show the contributions of the fundamental ( $\mathrm{TE}_{0}$-like) and second-order ( $\mathrm{TE}_{2}$-like) modes in blue and red, respectively. The first band (I) is completely formed by the fundamental mode, while the second and third bands (II and III) are a combination of both modes. A PBG is created between these two bands as a result of the anti-crossing point produced by the fundamental mode, folded into the first Brillouin zone, and the higher-order mode. In PhC theory, when two modes of the same polarization and parity intersect, they couple, and the bands repel [50], producing a dispersive behaviour similar to that obtained when a band reaches the edge of the irreducible Brillouin zone [51] $\left(k_{z}=0.52 \pi / a\right)$. Consequently, we obtain two different bimodal regimes near the PBG for the second and third bands, although in this work, we focus on the third band since lower group velocities can be achieved in this operating region. Therefore, in the third band (III), a bimodal behaviour with two different propagation constants and field patterns (see Fig. 1c) is obtained for wavelengths of $\sim 1550 \mathrm{~nm}$, which is the region of interest (ROI) for our purposes (shaded green area in Fig. 1b). The fundamental mode is strongly confined within the central waveguide of the $\mathrm{PhC}$, while the higher-order mode is partially localized in the transversal elements of the structure. Consequently, the higher-order mode strongly interacts with the periodic pattern of the structure, thus producing a highly dispersive behaviour of the third band 
a

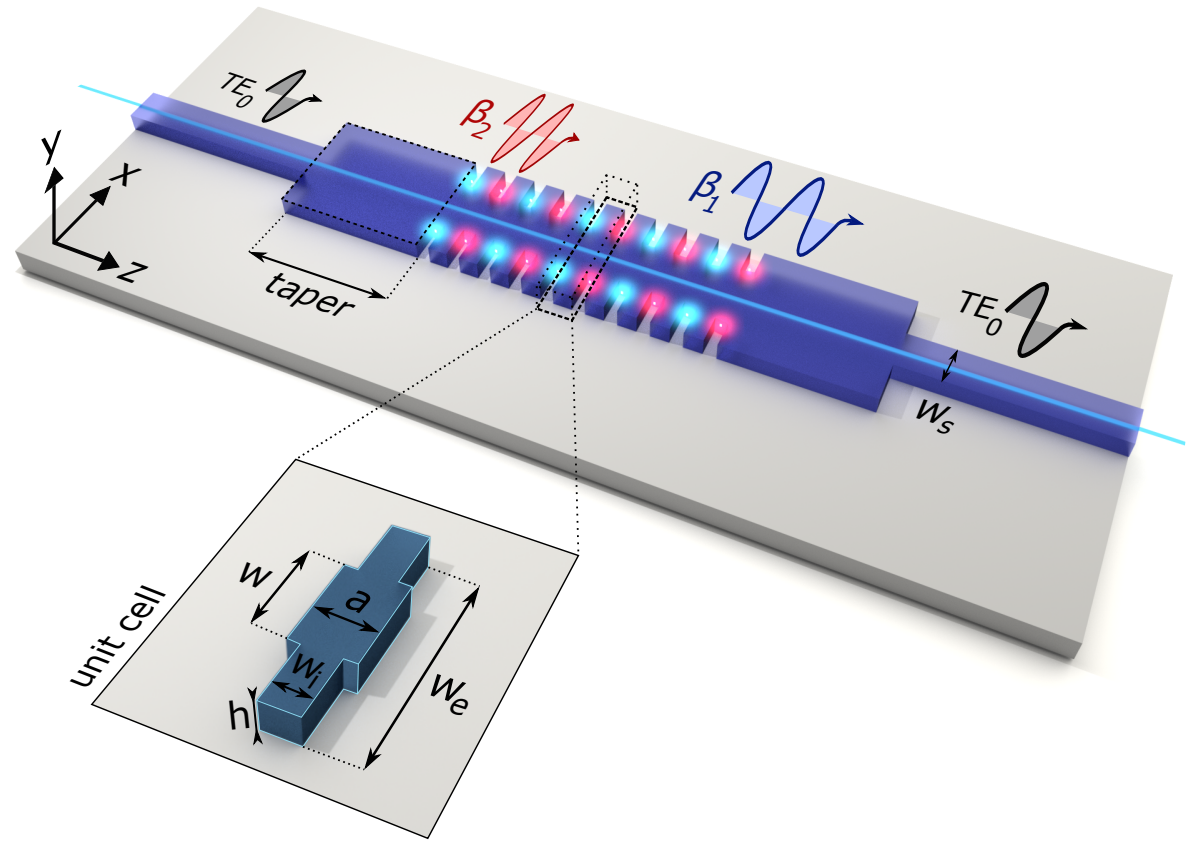

b

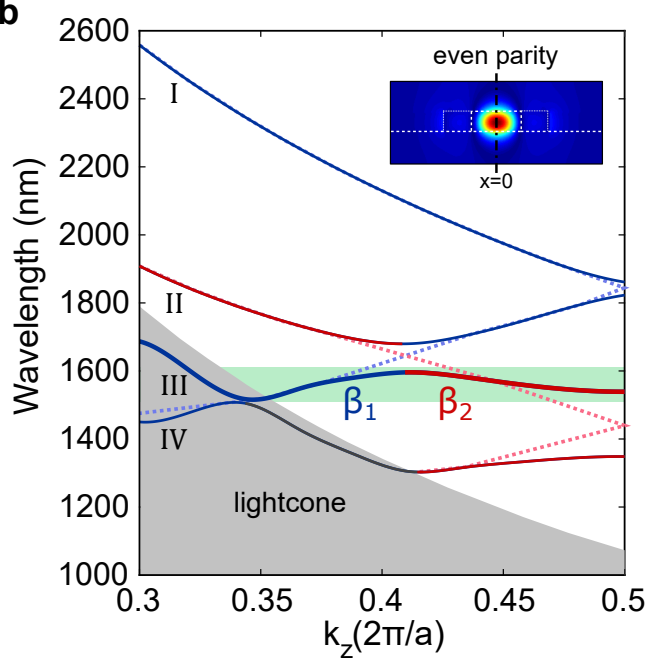

C
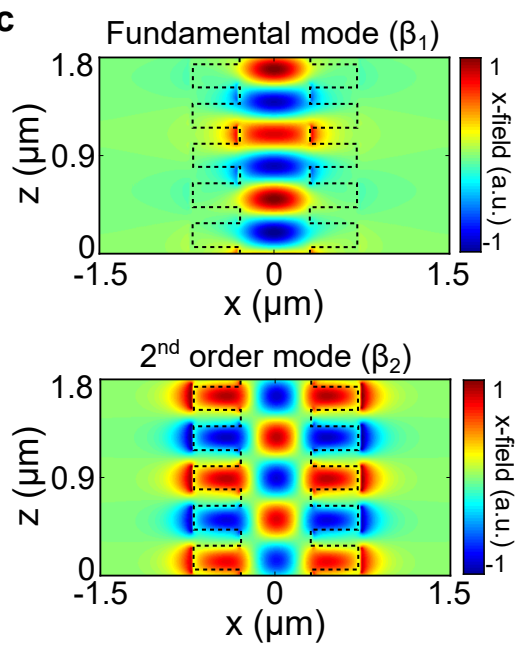

Fig. C.1. Design and simulation of the 1D PhC. (a) 3D sketch of the proposed design composed of two single-mode waveguides at the input/output ports and a rectangular taper between the uniform and periodic bimodal parts. The inset shows the unit cell dimensions of the 1D PhC. (b) Dispersion diagram of the 1D PhC showing the first three $x$-even parity bands for TE-like polarization. The blue lines depict the contribution of the fundamental mode to each band, and the red lines show the contribution of the higher-order mode. Dashed lines represent the hypothetical behaviour of the modes for a uniform non-periodic structure. The green shaded area indicates the bimodal region created in the proposed structure. The design parameters are $a=370$ $\mathrm{nm}$, transversal element width $w_{i}=220 \mathrm{~nm}$, transversal element length $w_{e}=1400 \mathrm{~nm}$, central waveguide width $w=600 \mathrm{~nm}$ and height $h=220 \mathrm{~nm}$, and single-mode waveguide width $w_{s}=$ $450 \mathrm{~nm}$. (c) Real part of the electric field $x$-component for the fundamental and higher-order modes in the $x z$ plane for $y=0$. The field patterns are calculated in the third band for $k_{z}=0.365$ $2 \pi / a$ and $k_{z}=0.47522 \pi / a$. The black dashed line represents the geometric shape of the silicon 1D PhC structure. 
at the end of the Brillouin zone. The group velocity, which is mathematically described as the derivative of the angular frequency with respect to the wave vector $\left(v_{g}=\delta w / \delta k\right)$ , is given by the slope of the bands for each $k_{z}=$ value in the dispersion diagrams previously shown. Therefore, at high wavelengths in the ROI, slow light is produced for both modes, which is not suitable for our purposes. In contrast, at lower wavelengths, only the higher-order mode becomes slow light as it nears the edge of the Brillouin zone, while the fundamental mode shows normal dispersive behaviour, thus achieving a high group velocity difference between both modes in this wavelength region.

Under induced RI variations in the system, the effective index of the higher-order mode will be drastically changed in comparison to the effective index of the fundamental mode, which acts as a reference. Accordingly, slowing the higher-order mode critically enhances the phase shift accumulated when a change in the RI is induced. Hence, an effect is obtained similar to what happens in an MZI when the arm length is increased to achieve higher phase shifts, but in this case, it drastically slows down the higher-order mode. To achieve this, we focus on studying the influence of the design parameters on the bimodal ROI in the third band. In Fig. 2a, we can observe the evolution of the dispersion relations for the second and third bands for different values of the parameter we. The rest of the design dimensions are kept as detailed above to obtain a bimodal behaviour around $1550 \mathrm{~nm}$. Figure 2a shows that as we increase we, the second and third bands are shifted to higher wavelengths. The higher-order mode contribution (red part of the bands) is clearly more sensitive to we variations than the fundamental mode (blue part of the bands) since the higher-order mode is more localized inside the transverse elements of the periodic structure. Having control of the higher-order-mode cut-off frequency enables us to design for which wavelengths the slow light effect of this mode occurs, thus obtaining the desired bimodal behaviour. Figure $2 \mathrm{~b}$ shows the maximum group velocity difference between both modes in the higher-order-mode slow light region and the bandwidth of the bimodal ROI as a function of we. As previously explained, the group velocity difference in the ROI increases with we until we reach wavelengths near the PBG, where the fundamental mode also becomes slow light. The bandwidth decreases due to the flattening of the higher-order mode, resulting in smaller wavelength bimodal regions. We selected a we value of $1400 \mathrm{~nm}$, as this configuration provides a high group velocity difference and a larger bandwidth. The critical effect of we can also be seen if we calculate the interference pattern of the spectrum as a sinusoidal function of the phase shift calculated from the bands in Fig. 2a and for a given length. In Fig. 2c, we can observe how we plays a crucial role in obtaining a large number of destructive and constructive peaks. With a we of $1300 \mathrm{~nm}$, only two interference peaks are formed in the ROI, while up to eight peaks are observed when this parameter is increased to $1400 \mathrm{~nm}$, as if it were an MZI with one of its arms drastically longer than the other. Moreover, the slow light influence of the 
higher-order mode on the interference pattern periodicity (see Fig. 2c) should be noted, where more grouped peaks are obtained for those wavelengths where the group velocity difference is maximized.

To study the interferometric behaviour of the device, the complete configuration with single-mode waveguides of $450 \mathrm{~nm}$ width as input and output ports was analysed. The transmission spectra for a length $N=150$ elements are depicted in Fig. 3a, with and without a rectangular taper of $1200 \mathrm{~nm}$ between the end of the single-mode waveguide and the bimodal periodic waveguide. It can be observed that the response is nearly flat when the taper is not present, since only the fundamental mode is propagated through the $1 \mathrm{D} \mathrm{PhC}$. With a rectangular taper, the higher-order mode is properly excited, and thus, the interference pattern is clearly observed. Moreover, as was previously shown in Fig. $2 c$, the interference pattern is more grouped at lower wavelengths, demonstrating the dispersive behaviour of the higher-order mode in this region. This behaviour appears for wavelengths of $\sim 1532 \mathrm{~nm}$ near the end of the irreducible Brillouin zone for the higher-order mode, where it becomes slow light. At even lower wavelengths (i.e., below $1530 \mathrm{~nm}$ ), only the fundamental mode propagates, and thus, a mono-modal response of the transmission spectrum is observed. The bimodal excitation is optimized for a range of taper lengths in Fig. 3b, calculated as the difference between a maximum and a minimum peak caused by modal interference. An optimal amplitude modulation of $\sim 70 \%$ for a taper of $1200 \mathrm{~nm}$ long is obtained for the modal interference nearest to the PBG. However, note that the bimodal excitation is decreased for lower wavelengths, as shown in the green shaded area of the ROI in Fig. 3a. To clarify the bimodal behaviour at the interfaces, the absolute value of the 2D-plane electric field at the output of the bimodal interferometer is depicted with and without a taper for destructive interference at $1563.5 \mathrm{~nm}$ in Fig. 3c and d, respectively. In the absence of a rectangular taper, all the energy is transferred to the single-mode output waveguide, while almost no power is transmitted to the output waveguide when the taper is present due to the proper bimodal excitation, which creates destructive interference.

To study the interferometric behaviour of the device, the complete configuration with single-mode waveguides of $450 \mathrm{~nm}$ width as input and output ports was analysed. The transmission spectra for a length $N=150$ elements are depicted in Fig. 3a, with and without a rectangular taper of $1200 \mathrm{~nm}$ between the end of the single-mode waveguide and the bimodal periodic waveguide. It can be observed that the response is nearly flat when the taper is not present, since only the fundamental mode is propagated through the 1D PhC. With a rectangular taper, the higher-order mode is properly excited, and thus, the interference pattern is clearly observed. Moreover, as was previously shown in Fig. 2c, the interference pattern is more grouped at lower wavelengths, demonstrating the dispersive behaviour of the higher-order mode in this region. This behaviour appears 


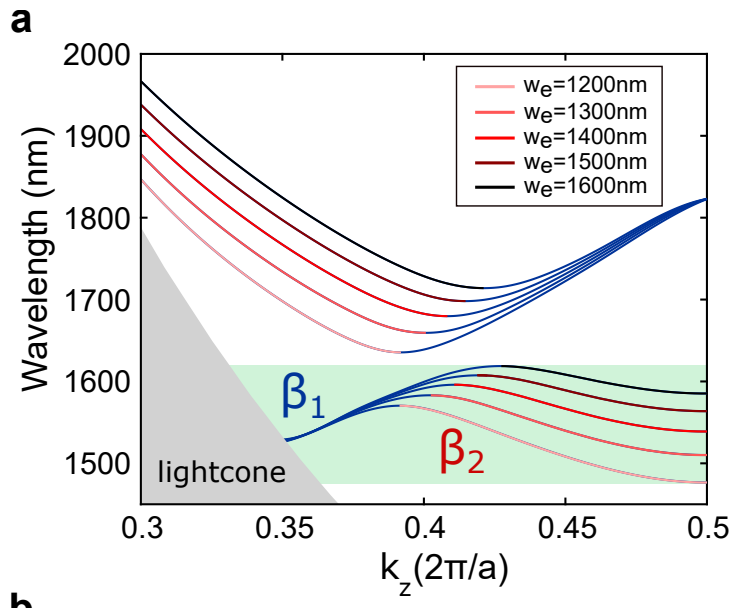

b
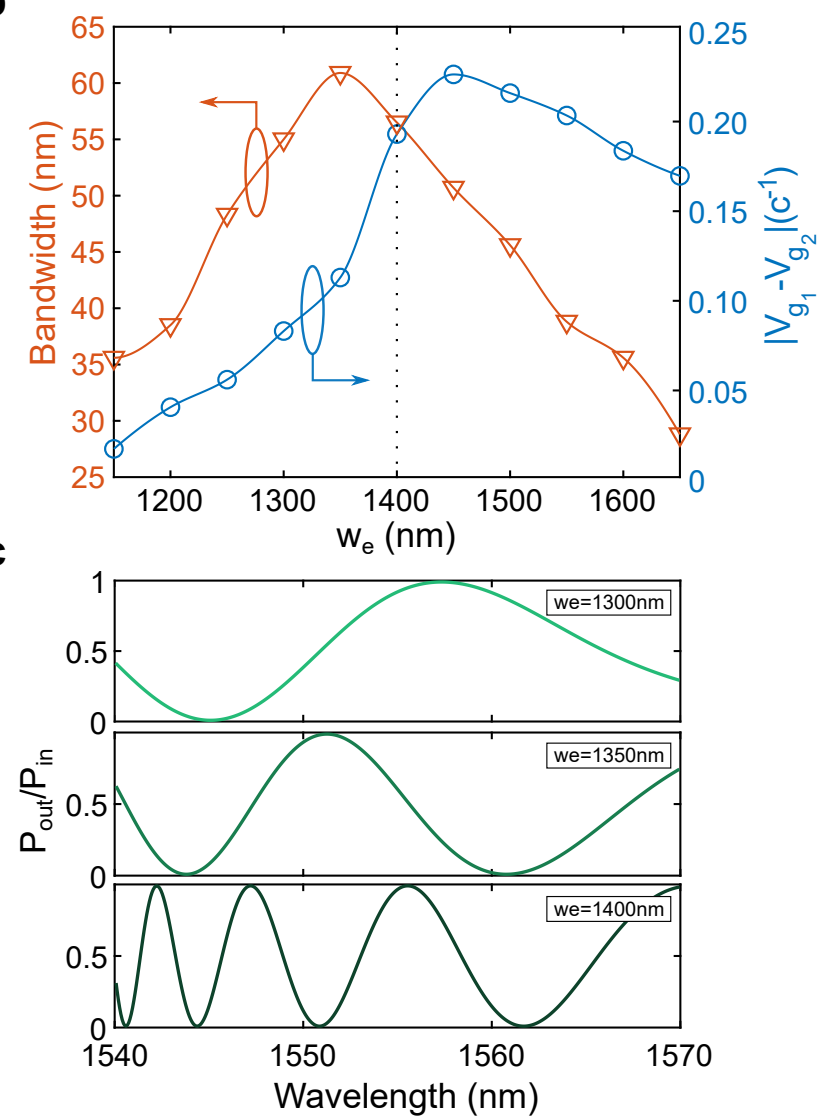

Fig. C.2. Optimization of the band structure. (a) Second and third bands for different transversal element lengths $w_{e}$ (the rest of the design parameters are the same as previously detailed). The contributions of the fundamental and higher-order modes to band formation in the bimodal region are depicted in blue and red, respectively. (b) Trade-off between the group velocity difference between both modes (in blue) and the bimodal bandwidth (in orange) as a function of $w_{e}$. The group velocity difference was calculated for those wavelengths in the lower edge of the bimodal ROI where a slow light behaviour is obtained for the higher order. (c) Ratio between the output and input power, calculated as the squared cosine of the phase shift as a function of the wavelength. Various we lengths are considered, representing the interference pattern evolution as a result of the slow light behaviour. 
for wavelengths of $\sim 1532 \mathrm{~nm}$ near the end of the irreducible Brillouin zone for the higher-order mode, where it becomes slow light. At even lower wavelengths (i.e., below $1530 \mathrm{~nm}$ ), only the fundamental mode propagates, and thus, a mono-modal response of the transmission spectrum is observed. The bimodal excitation is optimized for a range of taper lengths in Fig. 3b, calculated as the difference between a maximum and a minimum peak caused by modal interference. An optimal amplitude modulation of $\sim 70 \%$ for a taper of $1200 \mathrm{~nm}$ long is obtained for the modal interference nearest to the PBG. However, note that the bimodal excitation is decreased for lower wavelengths, as shown in the green shaded area of the ROI in Fig. 3a. To clarify the bimodal behaviour at the interfaces, the absolute value of the 2D-plane electric field at the output of the bimodal interferometer is depicted with and without a taper for destructive interference at $1563.5 \mathrm{~nm}$ in Fig. 3c and d, respectively. In the absence of a rectangular taper, all the energy is transferred to the single-mode output waveguide, while almost no power is transmitted to the output waveguide when the taper is present due to the proper bimodal excitation, which creates destructive interference.

To demonstrate that the fringes in the spectrum are due to modal interference, the 1D electric field $x$-component along the $z$-axis for $y=0$ in a $N=150$ bimodal periodic structure is calculated. By applying the Fast Fourier transform (FFT) over the propagating field, we obtain the wave vectors that are excited in the bimodal region. The results are shown in Fig. 3e as a function of the normalized wave vector axis and for different wavelengths. Each peak of the FFT corresponds to a propagating mode inside the periodic waveguide. As shown, the wave vectors of the modes inside the irreducible Brillouin zone are obtained, as well as those for the unfolded region between $k_{z}=0.5(2 \pi / a)$ and $k_{z}=0.7(2 \pi / a)$. This second Brillouin zone is a mirror image of the first region and provides information about the modes and their dispersion characteristics. Therefore, perfect agreement is obtained between the positions of the peaks and the band diagrams previously calculated by using two different simulation methods, which enables us to clarify the modal excitation inside the 1D PhC. Note that the fundamental mode at $k_{z}=$ $\sim 0.65(2 \pi / a)$ has a higher intensity than the higher-order mode at $k_{z}=\sim 0.45(2 \pi / a)$, which means that both modes are not equally excited and explains the results obtained in Fig. 3a regarding the interference pattern amplitude in the transmission spectrum.

\section{Experimental demonstration of slow light bimodal behaviour}

We fabricated bimodal interferometers with the design parameters previously detailed and a taper of nominal length $(1200 \mathrm{~nm})$ at the input-output interfaces (see Fig. 4a). All the parameters of the fabricated structures perfectly match the theoretical design except for the taper, which has a measured length of $\sim 1400 \mathrm{~nm}$. This difference occurs 
a
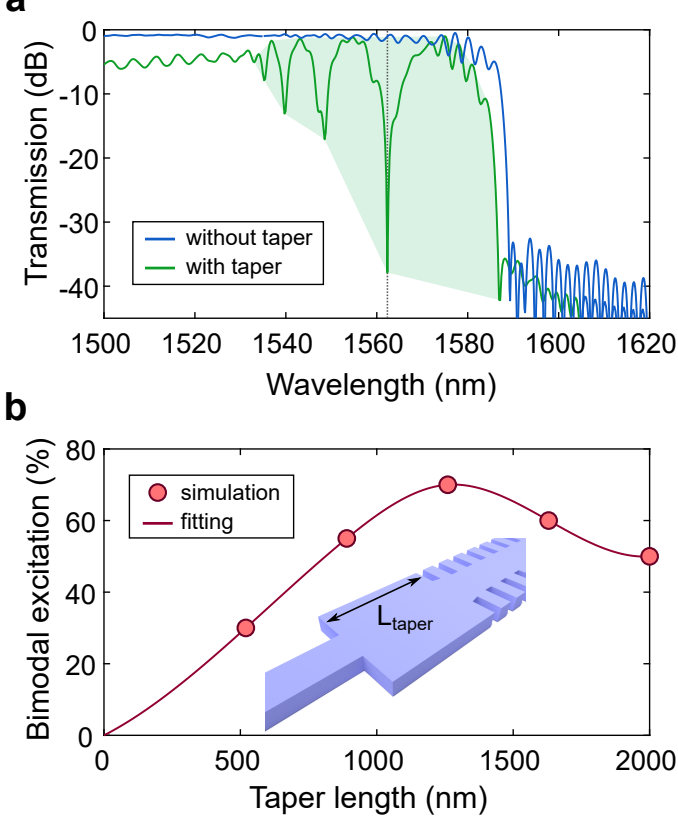

C

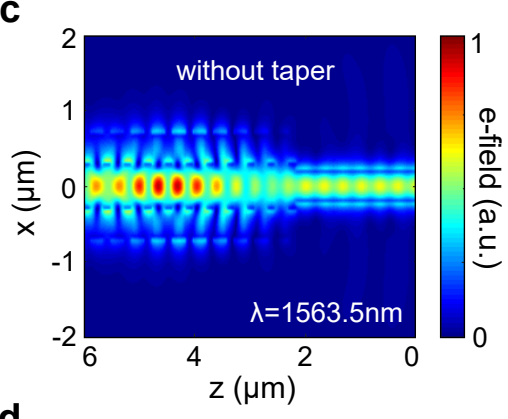

d

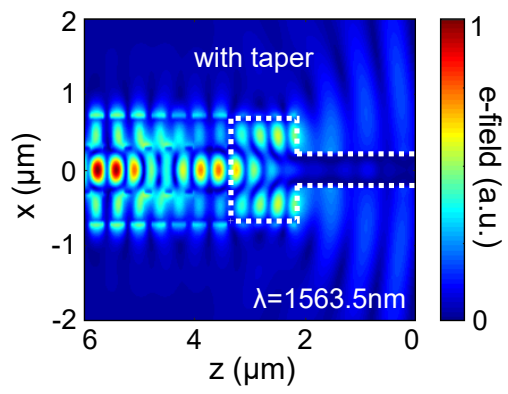

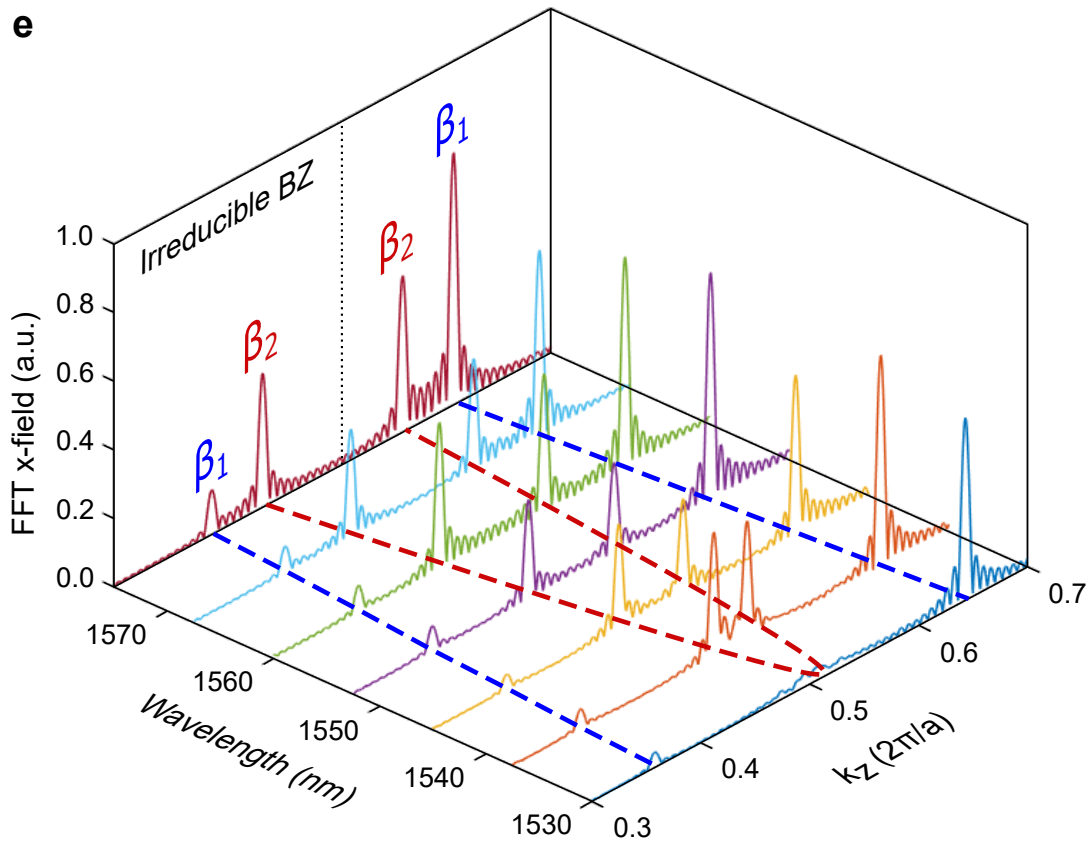

Fig. C.3. Simulation of the bimodal behaviour. (a) Transmission spectra for a bimodal PhC interferometer with and without a rectangular taper of $1200 \mathrm{~nm}$ with the previously detailed design parameters and for $N=150$ cell units repeated along the $z$-axis. The green shaded area represents the region of interest of the interferometer, corresponding to the bimodal region near the PBG. (b) Modal excitation obtained from the amplitude difference between constructive and destructive interference in the spectrum for different taper lengths. The inset details the value under consideration in a 3D sketch. (c,d) Electric field absolute value for the $x z$ plane and $y=0$ at the output interface between the bimodal periodic part and the single-mode waveguide with and without a rectangular taper between interfaces. (e) FFT amplitude of the electric field $x$-component along the propagation direction $z$ for different wavelengths in the bimodal region. The dashed blue and red lines in the wavelength $-k_{z}$ plane represent the dispersion relations of the fundamental and higher-order modes, respectively. The irreducible and second Brillouin zones are depicted. 
because the taper was very close to the first transversal element of the $1 \mathrm{D} \mathrm{PhC}$, with a gap of only $60 \mathrm{~nm}$ that was not resolved in the fabrication (see Fig. 4a). Despite this deviation, the fabricated taper length also remains within the optimal bimodal excitation range, as shown in Fig. 3b; thus, the experimental performance is not expected to be dramatically altered. Two different bimodal PhC lengths of $74 \mu \mathrm{m}$ and $148 \mu \mathrm{m}$ are considered, corresponding to numbers of periodic elements $N=200$ and $N=400$. The total length of the photonic sample is $0.9 \mathrm{~mm}$, corresponding to the on-chip length between the input-output grating couplers. The experimental transmission spectra for both configurations are depicted in Fig. 4b. A PBG for wavelengths higher than $1590 \mathrm{~nm}$ can be observed, below which the bimodal ROI is obtained, as was theoretically predicted in the band diagram and propagation simulations. The raw experimental spectra, as well as the filtered data, are depicted to distinguish the bimodal interferences from the ripple caused by Fabry-Perot resonances. The free spectral range (FSR) of the ripple is homogeneously distributed and very similar to that calculated for an on-chip cavity distance of $0.9 \mathrm{~mm}(\sim 1.5 \mathrm{~nm})$, which demonstrates that this is caused by the Fabry-Perot contribution. Moreover, the results show the same FSR for the two different bimodal lengths considered; hence, the ripple must be caused by a common optical cavity in both designs (i.e., the resonances between the access grating couplers).

The positions of the maximum and minimum oscillations originating from the bimodal constructive and destructive interferences, which are marked with circles in Fig. $4 \mathrm{~b}$, are obtained by applying Lorentzian fitting over the filtered spectra. As expected, a higher number of bimodal interferences is observed in the spectrum for the $N=400$ periods than for $N=200$ as a result of the increment of the physical optical length of the interferometer. To evaluate the slow light behaviour of a PhC in standard MZI configurations [7], the experimental group index of the active arm ( $\beta_{2}$ mode in our case) is calculated from the simulations of the reference arm ( $\beta_{12}$ mode). Therefore, the spectral dependence of the group index in the higher-order slow light mode can be deduced from the positions of the maximum $\lambda_{\max }$ and minimum $\lambda_{\min }$ spectral fringes as:

$$
n_{g}^{\beta 2}(\lambda)=\frac{\lambda_{\max } \lambda_{\min }}{2 L\left(\lambda_{\max }-\lambda_{\min }\right)}+n_{g}^{\beta 1}(\lambda)
$$

where $L$ is the length of the bimodal PhC waveguide and $n_{g}^{\beta 1}$ is the group index of the fundamental mode acting as a reference signal. The red and blue markers in Fig. 4c represent the experimental group index in the $N=200$ and $N=400$ configurations by using the equation described above. The bimodal slow light interferometer exhibits a group index up to $\sim 23$ for the higher-order mode, which perfectly matches the simulations. These simulated values were calculated from the propagation constants of both modes considered in the bimodal band region, demonstrating that the oscillations 
a

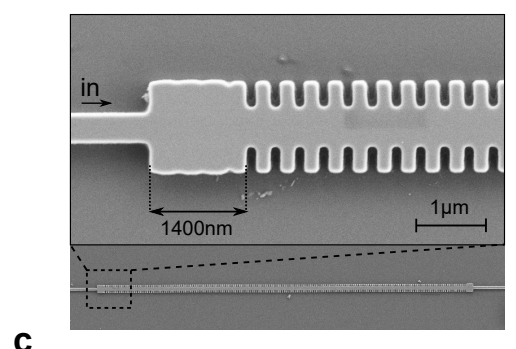

C

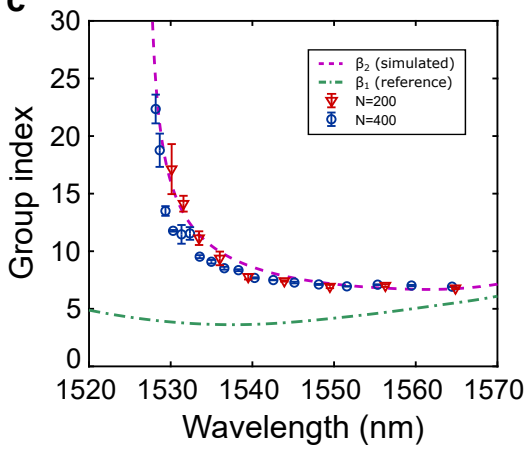

b

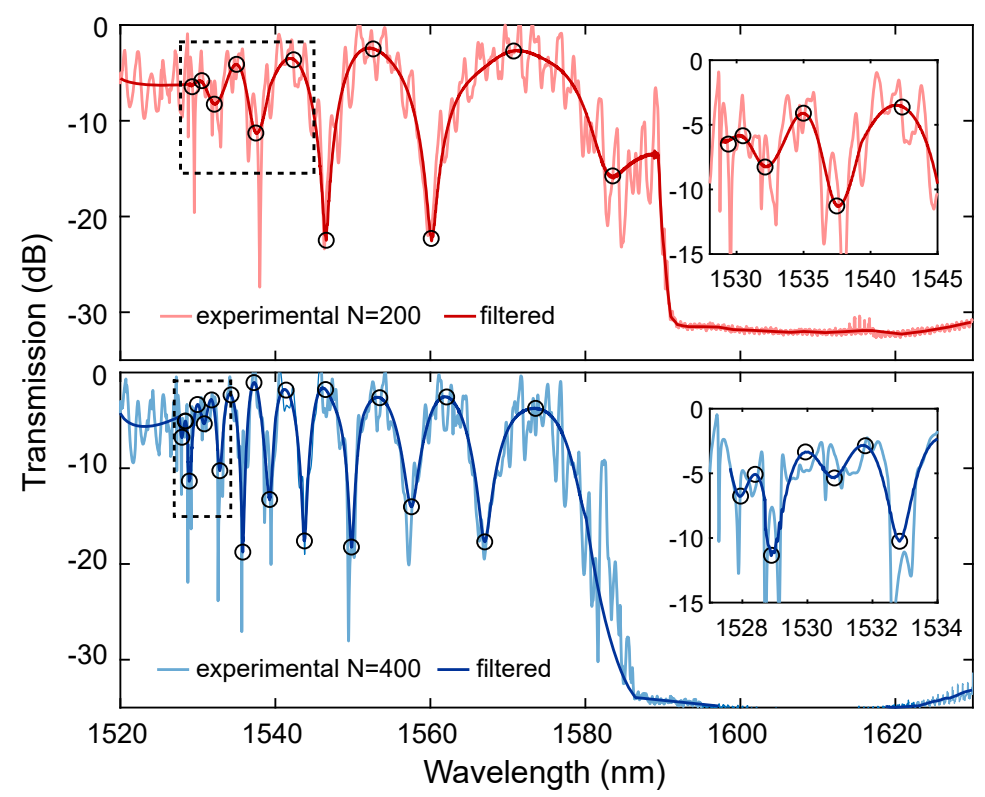

Fig. C.4. Experimental measurements of slow light bimodal behaviour. (a) Scanning electron microscopy (SEM) image of the fabricated silicon structures without a silica cover layer. A detailed picture of the interface between the single-mode and bimodal sections is shown with the presence of a fabricated rectangular taper of $\sim 1400 \mathrm{~nm}$ in length. (b) Normalized experimental transmitted spectra for $N=200$ and $N=400$ periods under a silica cover (upper and lower graphs, respectively). The spectra were normalized with respect to a reference uniform waveguide measured under the same environmental conditions. Light colours represent the raw experimental data; dark colours refer to the filtered signal without the Fabry-Perot ripple created in the photonic circuit cavities. Constructive and destructive interferences are indicated by the black circles in both graphs. Insets depict a close-up view of the transmission spectrum in the slow light region. (c) Group index experimentally obtained from the maxima and minima interference points and with the simulated fundamental mode group index as a reference. Three different experimental measurements were carried out to obtain the error bars, depicted as the data standard deviation. The dashed line represents the theoretical results obtained from the band diagram simulations, which were shifted by only $5 \mathrm{~nm}$ towards lower wavelengths to compensate for the small fabrication deviations.

marked with circles in Fig. $4 \mathrm{a}$ and $\mathrm{b}$ are due to the bimodal contribution. The simulated group index of the fundamental mode used in Eq. (1) is shown in green in Fig. 4c and presents lower group velocity values below 5 in the ROI.

\section{Interferometric performance in dynamic systems}

Once the static response is provided, to calculate the phase shift accumulated by an induced change in the RI, we must also consider the spectral shift of a given interference peak. Moreover, due to the dispersive behaviour of our proposed structure, the FSR 
caused by the bimodal interferences varies along the ROI, yielding lower values in the slow light region. Hence, the mean value between two contiguous FSRs is used to obtain the experimental phase shift, which is calculated as follows:

$$
\Delta \phi(\lambda)=\frac{2 \Delta \lambda}{\left(F S R_{H}+F S R_{L}\right)}
$$

where $\Delta \lambda$ is the wavelength shift of the minima produced for the induced RI changes and $F S R_{H, L}$ is the free spectral range at higher and lower wavelengths with respect to a given minimum interference peak, respectively.

To evaluate the response of the device as an optical modulator, a Peltier heater is used to change the chip operating temperature. Figure 5a shows the phase shift obtained as a function of the wavelength for different temperature increments in the $N=200$ and $N=400$ configurations. In both cases, the effect of slow light is clearly seen at wavelengths of $\sim 1530 \mathrm{~nm}$, where a drastic increase in the phase shift is obtained, which is also in good agreement with the simulations. Note that the $N=400$ interferometer results are twice as high as those of the shorter case due to double the length being used. A fitting of the phase shift evolution is depicted in Fig. $5 \mathrm{~b}$ for different destructive minima peaks when the temperature is varied, presenting perfectly linear behaviour for the available interferences. In the slow light region, phase shift values up to $\pi$ for temperature increments of $30^{\circ} \mathrm{C}$ are obtained at the $1532 \mathrm{~nm}$ interference, corresponding to a required silicon RI change of $5.4 \times 10^{-3}$ for a $L_{\pi}$ length of just $78 \mu \mathrm{m}$ (see dashed line in the upper graph of Fig. 5b). Likewise, for the $148-\mu \mathrm{m}$-long interferometer at $1529 \mathrm{~nm}, 13.2^{\circ} \mathrm{C}$ is required for a $\pi$ phase shift, corresponding to a required silicon RI change of $2.4 \times 10^{-3}$. By contrast, outside the slow light region, phase shift values of $\pi$ are obtained for a temperature change of $75^{\circ} \mathrm{C}$ at the $1550 \mathrm{~nm}$ interference in the $148-\mu \mathrm{m}$-long configuration, corresponding to a required silicon change in refractive index units (RIU) of $1.35 \times 10^{-2}$ (see dashed line in the lower graph of Fig. 5b). Nevertheless, even in this regime, efficient interferometers with a large bandwidth are proved for optical modulation, which also demonstrates scalable design flexibility depending on the desired operation.

Examples of the measured spectra for the different temperatures considered are shown in Fig. 5c for slow light and normal operations. The filtered signal is also depicted to clearly reveal the modulating response in the transmitted spectra between the on and off states. In addition, it should be noted that the insertion losses and the extinction ratio of the raw data depend strongly on the ripple of the Fabry-Perot resonances. Nonetheless, if we consider the filtered spectra as the contribution of the bimodal behaviour, the insertion losses are $\sim 2.5 \mathrm{~dB}$ for the upper graph in Fig. $5 \mathrm{c}$ and almost negligible in the case of the lower graph. In turn, an extinction ratio of $\sim 10 \mathrm{~dB}$ is obtained in the slow 
a
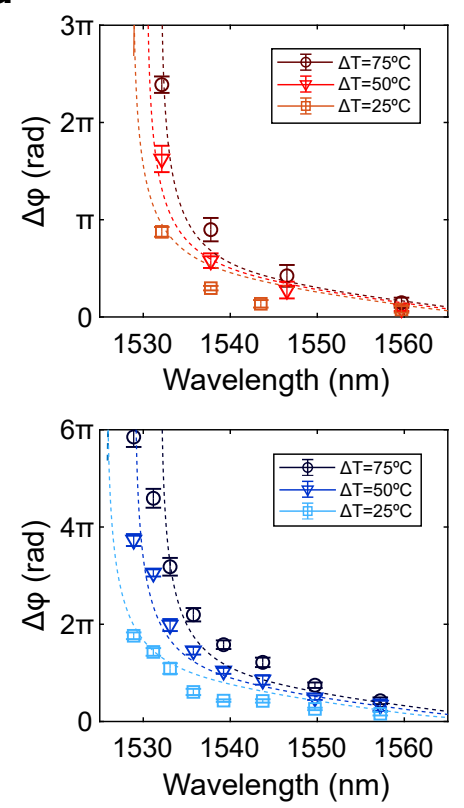

b
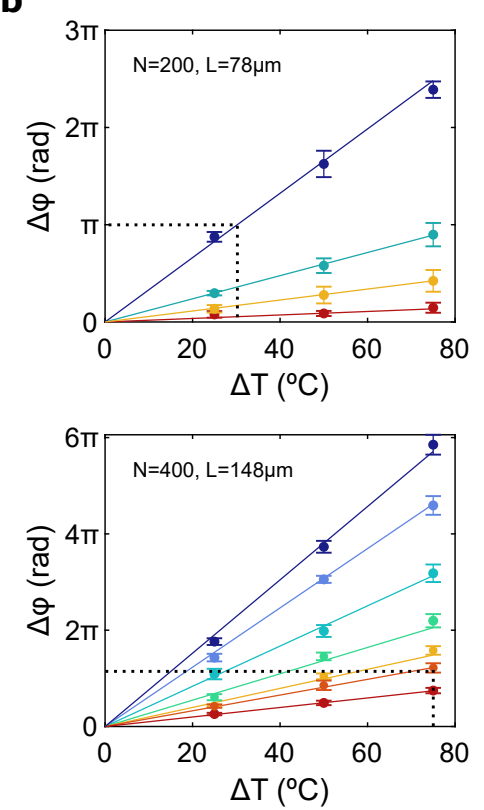

C
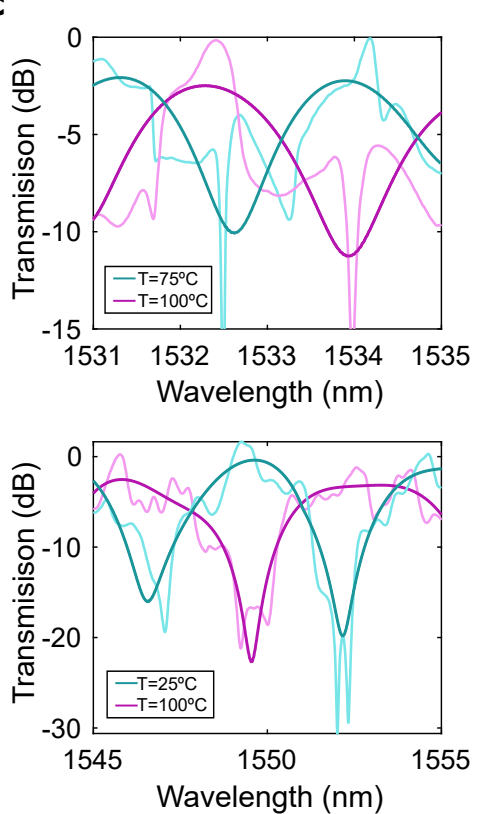

Fig. C.5. Optical response for changes in the silicon temperature. (a) Experimental phase shift obtained in the fabricated bimodal 1D PhC interferometer as a function of the wavelength for the different temperature increments considered and for the $N=200$ and $N=400$ configurations (upper and lower graphs, respectively). Dashed lines represent the band diagram simulation results, shifted $5 \mathrm{~nm}$ as in the previous case. (b) Evolution of the phase shift measured for a linear increment in the chip temperature. For the $N=200$ configuration in the upper graph, different minima interference wavelengths at $1559.68 \mathrm{~nm}, 1546.51 \mathrm{~nm}, 1537.76 \mathrm{~nm}$ and $1532.13 \mathrm{~nm}$ are shown, with the increase in phase sensitivity represented by the shift from red to blue. For the $N=$ 400 configuration, the minima interferences are placed at $1557.24 \mathrm{~nm}, 1549.73 \mathrm{~nm}, 1543.73 \mathrm{~nm}$, $1539.23 \mathrm{~nm}, 1535.75 \mathrm{~nm}, 1533.08 \mathrm{~nm}, 1530.54 \mathrm{~nm}$ and $1528.95 \mathrm{~nm}$ and are represented by the lines transitioning from red to blue in the lower graph. Five different experimental measurements were carried out to obtain the error bars depicted. (c) The upper graph represents a close-up view of the normalized transmission spectrum for the $N=200$ configuration (a bimodal 1D PhC length of $74 \mu \mathrm{m}$ ) working in the slow light region for a temperature change of $25{ }^{\circ} \mathrm{C}$, corresponding to an accumulated phase shift of $0.9 \pi$. The lower graph represents a close-up view of the normalized transmission spectrum for the $N=400$ configuration (a bimodal 1D PhC length of $148 \mu \mathrm{m}$ ) working in the normal light region for a temperature change of $75{ }^{\circ} \mathrm{C}$ and corresponding to an accumulated phase shift of $\pi$. For both graphs, raw and filtered data are represented by light and dark colours, respectively.

light region, which increases up to $\sim 20 \mathrm{~dB}$ outside this regime as a result of the improved bimodal excitation predicted theoretically.

Additional slow light bimodal structures without the silica upper cladding have also been fabricated to investigate the performance of the interferometer as a sensor. The design parameters are the same as those previously used to evaluate the operation as an optical modulator by means of temperature changes. Its transmission spectra when 
different ethanol dilutions in deionized water (DIW) are deposited over the 1D PhC interferometric structure are shown in Fig. 6a. Specifically, ethanol volumes in DIW of $3 \%, 6 \%$ and $9 \%$ are considered, corresponding to linear RI increments with respect to pure DIW of $1.6 \times 10^{-3}, 3.2 \times 10^{-3}$ and $4.8 \times 10^{-3}$ RIU according to the literature [52]. As can be observed, an interferometric response is now obtained for lower wavelengths (fringes located around $1515 \mathrm{~nm}$ are now tracked) since the DIW-based upper cladding has a lower RI than that of the silica. The spectrum is shifted towards higher wavelengths when increasing the RI of the cladding. As in the case of temperature changes, a Lorentzian fitting is processed over the spectral interferences to properly obtain the minima peaks caused by the bimodal behaviour (see Fig. 6a). Likewise, by knowing the spectral shift due to cladding RI changes, we can calculate its corresponding phase shift by using Eq. (2). Figure $6 \mathrm{~b}$ shows the accumulated phase shift for the interferences with the highest sensitivity in the slow light region for a linear increment in the cladding RI for both the $N=$ 200 and $N=400$ configurations. The experimental sensitivity can be determined as the slope of the phase shift fitting for different cladding RI changes. Experimental values up to $75.202 \pi \mathrm{rad} / \mathrm{RIU}$ and $150.832 \pi \mathrm{rad} / \mathrm{RIU}$ are obtained for bimodal interferometer lengths of $74 \mu \mathrm{m}$ and $148 \mu \mathrm{m}$, respectively. The obtained results clearly follow a linear evolution and present a twofold higher value due to the double length used for this configuration in comparison to the shorter one, similar to the previous case. In addition, the experimental results are compared to the simulations by applying the following equation to calculate the phase sensitivity of the interferometer:

$$
S(\lambda)=\frac{\Delta \phi}{\Delta n_{c}}=\frac{2 \pi L}{\lambda}\left(\frac{\delta n_{e f f 2}}{\delta n_{c}}-\frac{\delta n_{e f f 1}}{\delta n_{c}}\right)
$$

where $n_{c}$ is the cladding RI, $L$ is the interferometer length and $n_{e f f 1,2}$ is the effective index of the fundamental and higher-order modes, respectively. In standard MZI schemes in which one of the arms is completely isolated, the phase sensitivity is related only to the variation in the sensing arm effective index. In our case, both modes interact with the cladding variations; thus, the sensitivity depends on the effective index difference between them. Since the fundamental mode is strongly confined with a low group index and the higher-order mode presents a high dispersive behaviour, outstanding sensitivity values are obtained for the proposed bimodal $1 \mathrm{D} \mathrm{PhC}$ waveguide. The wavelength dependence of the sensitivity is depicted in Fig. 6c; as shown, its value dramatically increases in the slow light region, and perfect agreement with the simulations is presented. To compare these results with those of other interferometers in the literature with different lengths, values normalized to $1 \mathrm{~cm}$ are also calculated, corresponding to almost identical experimental sensitivities up to $10.62 \times 10^{3} 2 \pi \mathrm{rad} / \mathrm{RIUcm}$ and $10.19 \times 10^{3}$ $2 \pi \mathrm{rad} / \mathrm{RIUcm}$ for the $N=200$ and $N=400$ configurations, since they are normalized 
a

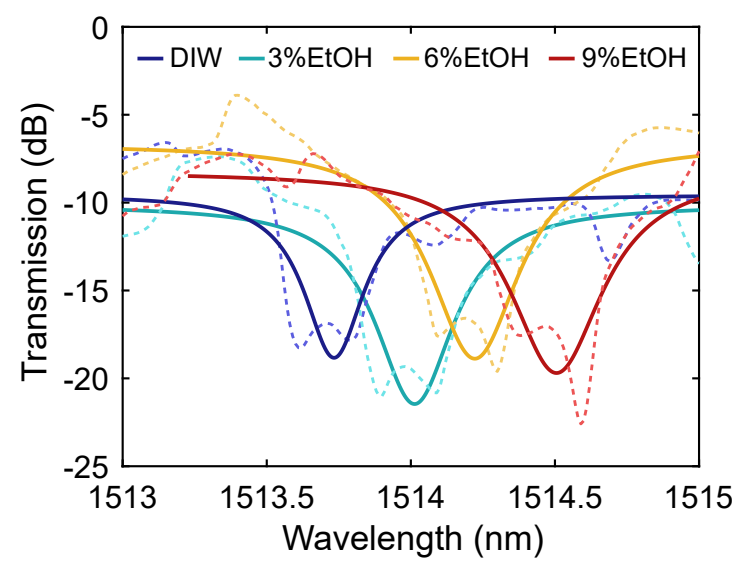

b

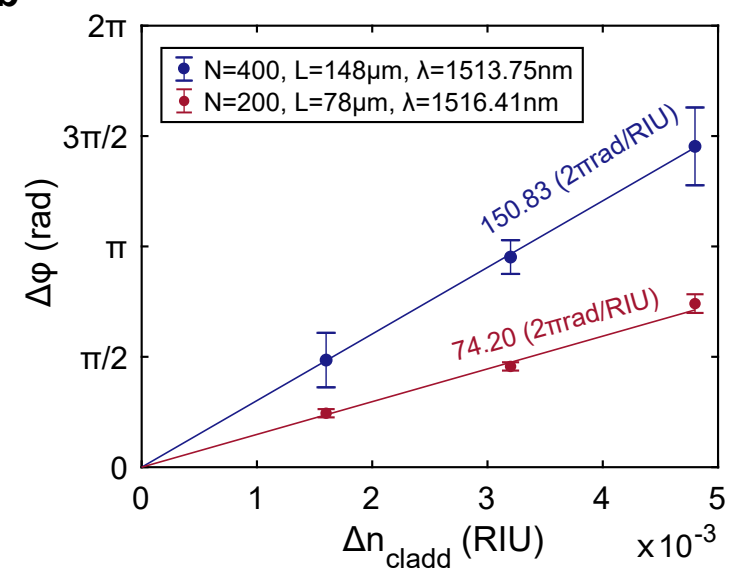

C

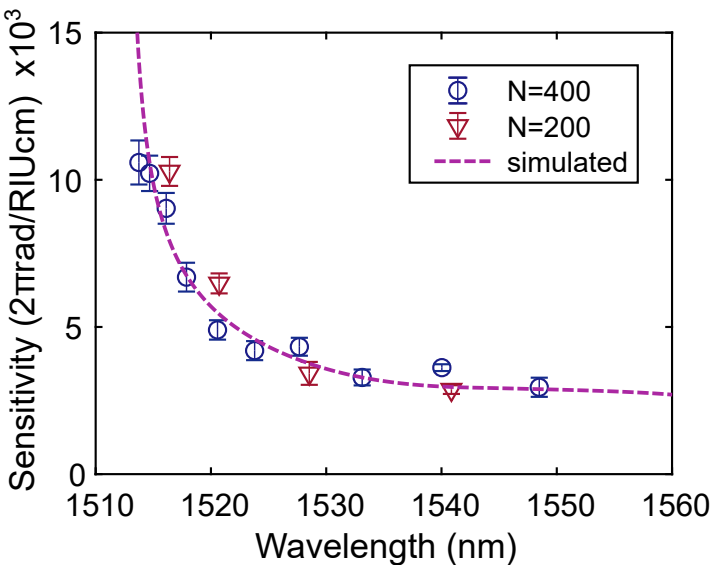

Fig. C.6. Optical response for changes in the cladding refractive index. (a) Normalized experimental spectra obtained for the $N=400$ configuration fabricated without a silica cover layer when the structure is covered with different ethanol concentrations in DIW dilutions. Raw data are depicted as dashed lines, while the Lorentzian fitting over the filtered spectra, removing the Fabry-Perot contribution, is represented as solid lines. (b) Phase shift measurements for both the $N=200$ and $N=400$ configurations as a function of the linear increments in the cladding RI. (c) RI sensitivity experimentally obtained for $N=200$ and $N=400$ bimodal 1D PhC sensors as the slope of the linear fitting for different changes in the cladding RI. These values are scaled to a $1 \mathrm{~cm}$ length to compare our results with the literature. The pink dashed line represents the simulated sensitivity curve obtained from the theoretical band structures under similar conditions. 
to a common length. Likewise, outside the slow light region, values around $3.5 \times 10^{3}$ $2 \pi \mathrm{rad} / \mathrm{RIUcm}$ for a large operation range of $30 \mathrm{~nm}$ are experimentally demonstrated, which are not as good as those in the slow light region but still higher than those of other interferometric configurations. In addition, the spectral sensitivity in nanometres per RI unit is calculated, yielding values of $138.75 \mathrm{~nm} / \mathrm{RIU}$ for those minima spectral dips occurring in the slow light regime shown in Fig. 6a. These values are in the range of the spectral sensitivities reported for similar slow light elements in the MZI configurations [45]. However, it should be noted that the spectral sensitivity may change outside the slow light regime due to the highly dispersive behaviour of the structure.

\subsubsection{Discussion}

In conclusion, we have proposed and demonstrated the possibility of obtaining optical interferometers by using a single-channel bimodal periodic structure. Compared to other bimodal configurations $[46,47,48,49]$, our design makes use of 1D PhC structures with an active mode working in the slow light regime, which is translated into a significantly higher phase-shift sensitivity to induced RI changes. This effect has been realized by engineering the periodic unit cell to achieve active control of the band structure to optimize the desired bimodal behaviour. An experimental group index of 23 was measured for the higher-order mode supported by the fabricated 1D PhC. This value is in the range of those results reported in other works in which similar 1D periodic structures were included in an MZI [44] but not as high as for works where 2D PhC structures were directly used to create an MZI [7]. This last result encourages us to look for other PhC configurations in which the single-channel bimodal slow wave behaviour can be further exploited to reduce the interferometer footprint and further improve the performance. Further optimization of the spectral response must be addressed in future experimental work for the current design, especially to reduce the Fabry-Perot ripple and improve the modulation depth and losses in the slow light regime.

The proposed device has also been experimentally validated for optical modulation and sensing purposes to determine its efficiency in dynamic systems. By changing the temperature of the chip, we can test the response of the interferometer to small changes in the RI of the silicon structure. In comparison with other interferometers that include slow light elements $[38,39]$, we propose a highly efficient temperature modulation where a change of only $30{ }^{\circ} \mathrm{C}$ is required to achieve phase shifts of $\pi$ in a single-channel interferometer with a footprint of only $\sim 100 \mu \mathrm{m}^{2}$. This size means a reduction of two orders of magnitude with respect to conventional MZI structures [28] and of more than one order of magnitude with respect to compact MZI modulators [29] and other interferometric schemes, including those based on the use of 1D and 
2D PhCs $[44,53]$. Due to its extremely reduced design, this type of bimodal PhC silicon waveguide may be used for the integration of multiple on-chip modulators [54], as well as for the implementation of matrix multiplications of several input binary signals for making all-optical programmable logic devices. However, switching speed limitations as a result of using thermo-optic effects [43] must be addressed in further developments. The sensing operation has also been demonstrated for different ethanol dilutions in DIW, corresponding to a linear change in the RI of the cladding. Experimental sensitivities of $10^{4} 2 \pi \mathrm{rad} / \mathrm{RIUcm}$ are reported, which indicate an improvement by a factor of more than 10 with respect to the traditional MZI configurations [30] and of $\sim 7.5$ for slot-based MZIs and silicon nitride bimodal waveguides [46,55]. These sensitivity results are similar to those obtained for other slow light MZI sensors [45] but in our case integrated in a significantly more compact single-channel structure. Furthermore, its straightforward monolithically formed design in silicon offers remarkable advantages for mass integration and low-cost production with significant implications for optical network interconnects or lab-on-a-chip instruments, among others.

\subsubsection{Materials and methods}

Numerical simulations to obtain the band diagrams of the basic $1 \mathrm{D}$ PhC unit cell were carried out using the MIT Photonics Band (MPB) free software, which computes definite-frequency eigenstates of Maxwell's equations in periodic dielectric structures. It employs the plane wave expansion (PWE) numerical method in fully vectorial and $3 \mathrm{D}$ spaces. In particular, we used silicon $(n=3.477)$ for the periodic structure, with a thermo-optic coefficient of $1.8 \times 10^{-4} / \mathrm{K}$, and silica $(n=1.444)$ for the substrate and cladding, with a grid step of $10 \mathrm{~nm}$, as the mesh. The first five TE-like bands were computed, including the first three that present an even parity with respect to the $x=$ 0 plane. The simulations of the transmission spectra and the field excitation were numerically calculated using the software package CST Microwave Studio. In more detail, we used a fully vectorial 3D time domain solver using finite-integration techniques to simulate the whole interferometric system, including the single-mode and bimodal waveguides. A hexahedral grid of 20 cells per wavelength was used for the entire structure, with silica as the background. In turn, the FFT of the field along the $z$-axis was obtained using 5000 points to obtain the propagating modes in the bimodal section. The excitation of the structure was provided by using standard waveguide ports at the input and output to provide the scattering parameters.

The photonic structures were fabricated on a silicon-on-insulator (SOI) wafer with a silicon layer thickness of $220 \mathrm{~nm}$ and a silica buried layer of $2 \mu \mathrm{m}$. An acceleration voltage of $30 \mathrm{KeV}$ and an aperture size of $30 \mu \mathrm{m}$ were used in an electron-beam lithography 
process to create the photonic structures on a hydrogen silsesquioxane (HSQ) negative resist, and then inductively coupled plasma etching was used to transfer the designed patterns onto the silicon layer. For the experimental characterization, a continuous wave (CW) tuneable laser (Keysight 81980) and a coherent TE polarizer were used to vertically couple the light into photonic structures by using cleaved optical fibres close to the grating couplers. At the output, a power metre (Keysight 81636B) synchronized with the laser measured the response of the optical circuits. The transmitted spectra were digitally recorded using a LabVIEW application, which was also responsible for controlling the chip temperature by using a Peltier heater connected to the copper holder of the photonic sample. A time frame of 5 min was used after the temperature changes to let the sample stabilize at the desired conditions. For the sensing experiments, the chip was placed on an airtight container and covered with increasing ethanol in DIW dilutions directly dropped onto the sample and measured after a 5-min stabilization period.

\section{Acknowledgements}

The authors acknowledge funding from the Generalitat Valenciana through the AVANTI/2019/123, ACIF/2019/009 and PPC/2020/037 grants and from the European Union through the operational program of the European Regional Development Fund (FEDER) of the Valencia Regional Government 2014-2020. We also thank Pablo Sanchis and Irene Olivares for their helpful discussions and assistance.

\section{Author contributions}

The idea and its development were conceived by L.T.M. and J.G.R. The chip fabrication processes were carried out by A.G. under the supervision of L.T.M. and J.G.R. The optical characterizations and experiments were performed by L.T.M. The data were studied and analysed by L.T.M. and J.G.R. The manuscript was written by L.T.M. and J.G.R. The authors declare that they have no conflict of interest.

\subsubsection{References}

1. Lorentz, H. A. The Theory of Electrons and Its Applications to the Phenomena of Light and Radiant Heat. (New York: Columbia University Press, 1909).

2. Hau, L. V. et al. Light speed reduction to 17 metres per second in an ultracold atomic gas. Nature 397, 594-598 (1999). 
3. Bigelow, M. S., Lepeshkin, N. N. \& Boyd, R. W. Observation of ultraslow light propagation in a ruby crystal at room temperature. Physical Review Letters 90, 113903 (2003).

4. Bigelow, M. S., Lepeshkin, N. N. \& Boyd, R. W. Superluminal and slow light propagation in a room-temperature solid. Science 301, 200-202 (2003).

5. Joannopoulos, J. D., Villeneuve, P. R. \& Fan, S. H. Photonic crystals: putting a new twist on light. Nature 386, 143-149 (1997).

6. Joannopoulos, J. D. et al. Photonic Crystals: Molding the Flow of Light. 2nd edn. (Princeton, NJ, USA: Princeton University Press, 2008).

7. Vlasov, Y. A. et al. Active control of slow light on a chip with photonic crystal waveguides. Nature 438, 65-69 (2005).

8. Krauss, T. F. Why do we need slow light? Nature Photonics 2, 448-450 (2008).

9. Baba, T. Slow light in photonic crystals. Nature Photonics 2, 465-473 (2008).

10. Krauss, T. F. Slow light in photonic crystal waveguides. Journal of Physics D: Applied Physics 40, 2666-2670 (2007).

11. Noda, S. et al. Full three-dimensional photonic bandgap crystals at near-infrared wavelengths. Science 289, 604-606 (2000).

12. Beggs, D. M. et al. Ultracompact and low-power optical switch based on silicon photonic crystals. Optics Letters 33, 147-149 (2008).

13. Lee, M. R. \& Fauchet, P. M. Two-dimensional silicon photonic crystal based biosensing platform for protein detection. Optics Express 15, 4530-4535 (2007).

14. Liberal, I. \& Engheta, N. Near-zero refractive index photonics. Nature Photonics 11, 149-158 (2017).

15. Notomi, M. Manipulating light with strongly modulated photonic crystals. Rep. Prog. Phys. 73, 096501 (2010).

16. Centini, M. et al. Dispersive properties of finite, one-dimensional photonic band gap structures: applications to nonlinear quadratic interactions. Physical Review E 60, 4891-4898 (1999).

17. Scalora, M. et al. Ultrashort pulse propagation at the photonic band edge: large tunable group delay with minimal distortion and loss. Physical Review E 54, R1078-R1081 (1996). 
18. Yun, T. Y. \& Chang, K. Uniplanar one-dimensional photonic-bandgap structures and resonators. IEEE Transactions on Microwave Theory and Techniques 49, 549-553 (2001).

19. Hopman, W. C. L. et al. Quasi-one-dimensional photonic crystal as a compact building-block for refractometric optical sensors. IEEE Journal of Selected Topics in Quantum Electronics 11, 11-16 (2005).

20. Povinelli, M. L., Johnson, S. G. \& Joannopoulos, J. D. Slow-light, band-edge waveguides for tunable time delays. Optics Express 13, 7145-7159 (2005).

21. Hwang, R. B. Negative group velocity and anomalous transmission in a one-dimensionally periodic waveguide. IEEE Transactions on Antennas and Propagation 54, 755-760 (2006).

22. Gnan, M. et al. Fabrication of low-loss photonic wires in silicon-on-insulator using hydrogen silsesquioxane electron-beam resist. Electronics Letters 44, 115-116 (2008).

23. Ma, Y. M. et al. Mid-infrared slow light engineering and tuning in 1-D grating waveguide. IEEE Journal of Selected Topics in Quantum Electronics 24, 6101608 (2018).

24. Sabek, J. et al. Experimental study of an evanescent-field biosensor based on 1D photonic bandgap structures. Beilstein Journal of Nanotechnology 10, 967-974 (2019).

25. Mathias, P. C., Ganesh, N. \& Cunningham, B. T. Application of photonic crystal enhanced fluorescence to a cytokine immunoassay. Analytical Chemistry 80, 9013-9020 (2008).

26. Treyz, G. V., May, P. G. \& Halbout, J. M. Silicon Mach-Zehnder waveguide interferometers based on the plasma dispersion effect. Applied Physics Letters 59, 771-773 (1991).

27. Heideman, R. G., Kooyman, R. P. H. \& Greve, J. Performance of a highly sensitive optical waveguide Mach-Zehnder interferometer immunosensor. Sensors and Actuators B: Chemical 10, 209-217 (1993).

28. Liao, L. et al. High speed silicon Mach-Zehnder modulator. Optics Express 13, 3129-3135 (2005).

29. Green, W. M. J. et al. Ultra-compact, low RF power, $10 \mathrm{~Gb} / \mathrm{s}$ silicon Mach-Zehnder modulator. Optics Express 15, 17106-17113 (2007).

30. Prieto, F. et al. An integrated optical interferometric nanodevice based on silicon technology for biosensor applications. Nanotechnology 14, 907-912 (2003). 
31. Melikyan, A. et al. High-speed plasmonic phase modulators. Nature Photonics 8, 229-233 (2014).

32. Haffner, C. et al. All-plasmonic Mach-Zehnder modulator enabling optical high-speed communication at the microscale. Nature Photonics 9, 525-528 (2015).

33. Ayata, M. et al. High-speed plasmonic modulator in a single metal layer. Science 358, 630-632 (2017).

34. Gao, Y. K. et al. Plasmonic Mach-Zehnder interferometer for ultrasensitive on-chip biosensing. ACS Nano 5, 9836-9844 (2011).

35. Liu, M. et al. A graphene-based broadband optical modulator. Nature 474, 64-67 (2011).

36. Sorger, V. J. et al. Ultra-compact silicon nanophotonic modulator with broadband response. Nanophotonics 1, 17-22 (2012).

37. Wang, C. et al. Integrated lithium niobate electro-optic modulators operating at CMOS-compatible voltages. Nature 562, 101-104 (2018).

38. Shaw, N. et al. Optical slow-wave resonant modulation in electro-optic GaAs/AlGaAs modulators. Electronics Letters 35, 1557-1558 (1999).

39. Camargo, E. A., Chong, H. M. H. \& De La Rue, R. M. Highly compact asymmetric Mach-Zehnder device based on channel guides in a two-dimensional photonic crystal. Applied Optics 45, 6507-6510 (2006).

40. Brosi, J. M. et al. High-speed low-voltage electro-optic modulator with a polymer-infiltrated silicon photonic crystal waveguide. Optics Express 16, 4177-4191 (2008).

41. Tanabe, T. et al. Low power and fast electro-optic silicon modulator with lateral p-i-n embedded photonic crystal nanocavity. Optics Express 17, 22505-22513 (2009).

42. Chong, H. M. H. \& De La Rue, R. M. Tuning of photonic crystal waveguide microcavity by thermooptic effect. IEEE Photonics Technology Letters 16, 1528-1530 (2004).

43. Geis, M. W. et al. Submicrosecond submilliwatt silicon-on-insulator thermooptic switch. IEEE Photonics Technology Letters 16, 2514-2516 (2004).

44. Brimont, A. et al. High speed silicon electro-optical modulators enhanced via slow light propagation. Optics Express 19, 20876-20885 (2011).

45. Qin, K. et al. Slow light Mach-Zehnder interferometer as label-free biosensor with scalable sensitivity. Optics Letters 41, 753-756 (2016). 
46. Zinoviev, K. E. et al. Integrated bimodal waveguide interferometric biosensor for label-free analysis. Journal of Lightwave Technology 29, 1926-1930 (2011).

47. Duval, D. et al. Nanophotonic lab-on-a-chip platforms including novel bimodal interferometers, microfluidics and grating couplers. Lab on a Chip 12, 1987-1994 (2012).

48. Torrijos-Morán, L. \& García-Rupérez, J. Single-channel bimodal interferometric sensor using subwavelength structures. Optics Express 27, 8168-8179 (2019).

49. Torrijos-Morán, L., Griol, A. \& García-Rupérez, J. Experimental study of subwavelength grating bimodal waveguides as ultrasensitive interferometric sensors. Optics Letters 44, 4702-4705 (2019).

50. Notomi, M. et al. Extremely large group-velocity dispersion of line-defect waveguides in photonic crystal slabs. Physical Review Letters 87, 253902 (2001).

51. Olivier, S. et al. Mini-stopbands of a one-dimensional system: the channel waveguide in a two-dimensional photonic crystal. Physical Review B 63, 113311 (2001).

52. García-Rupérez, J. et al. Label-free antibody detection using band edge fringes in SOI planar photonic crystal waveguides in the slow-light regime. Optics Express 18, 24276-24286 (2010).

53. Soljačić, M. et al. Photonic-crystal slow-light enhancement of nonlinear phase sensitivity. Journal of the Optical Society of America B 19, 2052-2059 (2002).

54. Reed, G. T. et al. Silicon optical modulators. Nature Photonics 4, 518-526 (2010).

55. Liu, Q. et al. Highly sensitive Mach-Zehnder interferometer biosensor based on silicon nitride slot waveguide. Sensors and Actuators B: Chemical 188, 681-688 (2013). 


\title{
4.2 Paper D:
}

Journal of Lightwave Technology 39, 3495-3501 (2021)

\section{Ultra-compact optical switches using slow light bimodal silicon waveguides}

\author{
Luis Torrijos-Morán, Antoine Brimont, Amadeu Griol, \\ Pablo Sanchis and Jaime García-Rupérez
}

(C) 2021 Institute of Electrical and Electronics Engineers (IEEE) and Optical Society of America (OSA) under a Creative Commons Attribution 4.0 International License. 



\title{
Ultra-compact optical switches using slow light bimodal silicon waveguides
}

\author{
Luis Torrijos-Morán, Antoine Brimont, Amadeu Griol, \\ Pablo Sanchis and Jaime García-Rupérez
}

\begin{abstract}
Nanophotonics Technology Center, Universitat Politècnica de València, Camino de Vera s/n, 46022 Valencia, Spain
\end{abstract}

\begin{abstract}
Switches are essential components in several optical applications, in which reduced footprints are highly desirable for mass production of densely integrated circuits at low cost. However, most conventional solutions rely on making long switching structures, thus increasing the final device size. Here, we propose and experimentally demonstrate an ultra-compact 2x2 optical switch based on slow-light-enhanced bimodal interferometry in one-dimensional silicon photonic crystals. By properly designing the band structure, the device exhibits a large group index contrast between the fundamental even mode and a higher order odd mode for TE polarization. Thereby, highly dispersive and broadband bimodal regions for high-performance operation are engineered by exploiting the different symmetry of the modes. Two configurations are considered in the experiments to analyze the dimensions influence on the switching efficiency. As a result, a photonic switch based on a bimodal single-channel interferometer with a footprint of only $63 \mu \mathrm{m}^{2}$, a power consumption of $19.5 \mathrm{~mW}$ and a crosstalk of $15 \mathrm{~dB}$ is demonstrated for thermo-optic tunability.
\end{abstract}

\subsubsection{Introduction}

Switches play a prominent role in current communication networks to address the ever-growing increase in the data centers traffic [1]. In this context, photonics offers remarkable advantages in terms of low power consumption and high speed operation for switching applications [2], [3]. Switching light is usually accomplished by interference between signals that have propagated along different optical paths. To this end, the most common photonic structures employed are ring resonators and Mach-Zehnder interferometers (MZIs). Resonant schemes allow very small footprints but at expenses of decreasing the optical bandwidth [4], [5]. In turn, MZI switches offer a broader bandwidth as well as a higher tolerance to fabrication deviations, although long optical paths that increase the resulting footprint are required [6]-[8]. Optical switches based on multimode interferometers (MMIs) exhibit both a broadband operation and robustness[9]. However, 
these types of structures suffer from a higher power consumption, thus requiring further improvement in the heaters geometry and fabrication to design short devices [10].

On the other hand, ultra-compact phase shifters are possible by integrating materials with unique optical properties in silicon. Transparent conducting oxides, such as Indium Tin Oxide (ITO), have been used either as a more efficient heater [11] or in hybrid photonic waveguide as an active material [12]- [14]. In the latter, phase shifter lengths in the micrometer range have been demonstrated but with high insertion losses. Thereby, the replacement of ITO by high-mobility transparent conducting oxides such as cadmium oxide have been proposed to reduce optical losses [15], [16]. Alternatively, photonic crystals (PhCs) have also been largely investigated for thermooptic switching with ultra-compact lengths and low powerconsumption [17]. PhCs allow to drastically reduce the group velocity of the propagating mode, the so-called slow light phenomenon, thus increasing the optical length interaction while the physical length remains small [18]-[20]. This effect is exploited, for instance, to develop 2D hole patterned array PhC structures for all-optical switching based on high speed MZIs [21], or for thermo-optic effects in high-performance MMIs [22] and ultra-compact directional couplers of less than $100 \mu \mathrm{m}^{2}$ footprint [23], [24]. 2D PhCs configurations have also been used to develop optical microcavities for extremely low consumption at the sub-femtojoule level [25]. However, decreasing the group velocity also makes light coupling to the $\mathrm{PhC}$ modes to be more inefficient and reduces the optical bandwidth, which must be addressed when using slow light structures [26].

Due to its structural complexity, including 2D PhCs adds extra difficulties in micro-structuring optical chips, which hinders fabrication processes [27]. Accordingly, 1D PhCs present more straightforward designs suitable for mass-level production, while preserving the slow light advantages from compactness [28]. In addition, this type of structures does not need additional periodic cells in the transverse spatial direction, thus reducing the lateral size and the footprint of the device 1D PhCs have been demonstrated for tunable delay lines [29], refractometric sensing [30], negative group velocity phenomena [31], slow light engineering waveguides [32] and biosensing [33], among others. Accordingly, high speed electro-optic modulators based on 1D PhCs in MZI configurations have also been demonstrated [34], although the interferometric structure still poses a limit on the minimum achievable footprint. To overcome some of these size limitations, single-channel structures based on bimodal interferometry in periodic subwavelength waveguides are proposed for the development of ultra-sensitive sensors [35], [36]. Furthermore, bimodal 1D PhC waveguides working in the slow light regime have also been designed as ultra-compact modulators with footprints of only 100 $\mu \mathrm{m}^{2}$ [37]. In these last structures, the operation is based on the interference between two modes of the same polarization and parity in a singlechannel silicon structure, with 
a large group velocity difference and without the need of additional structures or other materials.

In this paper, we propose the use of bimodal $1 \mathrm{D} \mathrm{PhC}$ waveguides based on silicon as highly efficient and ultracompact 2x2 optical switches. We optimize the periodic unit cell of the 1D PhC to support two TE-like modes of both even and odd symmetry, designed with a large group velocity difference. The device is experimentally demonstrated for thermo-optic switching with a very good agreement with simulations. Our design encompasses the benefits from 1D PhCs and bimodal waveguides, to validate this type of structures for optical switching with extremely reduced footprints.

\subsubsection{Concept and design}

The proposed design of the device is presented in Fig. 1, with a periodic bimodal waveguide acting as the switching structure and four single-mode silicon waveguides acting as input and output ports. The unit cell of the periodic bimodal section is designed to be within the photonic crystal regime and to support the fundamental even mode and a higher order odd mode at the transversal plane, both for the transverse-electric (TE) polarization. Due to symmetry conditions, the fundamental mode of the displaced single-mode input waveguide excites the even and odd parity modes in the bimodal section, which propagate and interfere at the abrupt discontinuity with the single-mode output waveguides. Therefore, the transferred power may be expressed as a function of the phase shift accumulated between these two modes in the bimodal section, thus creating an interference pattern in the transmitted spectra. In addition, a rectangular taper section for an efficient modal excitation is placed at the interface between the access ports and the bimodal waveguide.

In PhC theory, when two modes of the same polarization and parity are expected to intersect, they couple in the same band and form an anti-crossing point [37], [38]. In contrast, modes of different parity do not interact in the band formation, so that they can be overlapped with different dispersion properties. Consequently, using modes of even and odd symmetry provides an additional degree of freedom in obtaining broadband and highly dispersive bimodal regions, since their dispersion relationships can be designed independently by a proper control of the unit cell dimensions. Figure 2(a) shows the first seven bands for a unit cell with pitch $a=370 \mathrm{~nm}$, transversal element width $w_{i}=220$ $\mathrm{nm}$, transversal element length $w_{e}=1700 \mathrm{~nm}$ and central waveguide width $w=650 \mathrm{~nm}$, everything in a fully etched silicon layer of $220 \mathrm{~nm}$ thickness covered by a silica cladding. The rest of the design parameters considered are single-mode waveguide width $w_{s}=450$ $\mathrm{nm}$, taper length $t=1200 \mathrm{~nm}$ and distance between the ports $d=300 \mathrm{~nm}$. To obtain the bands structure of the 1D PhC, definite-frequency eigenstates of Maxwell's equations 


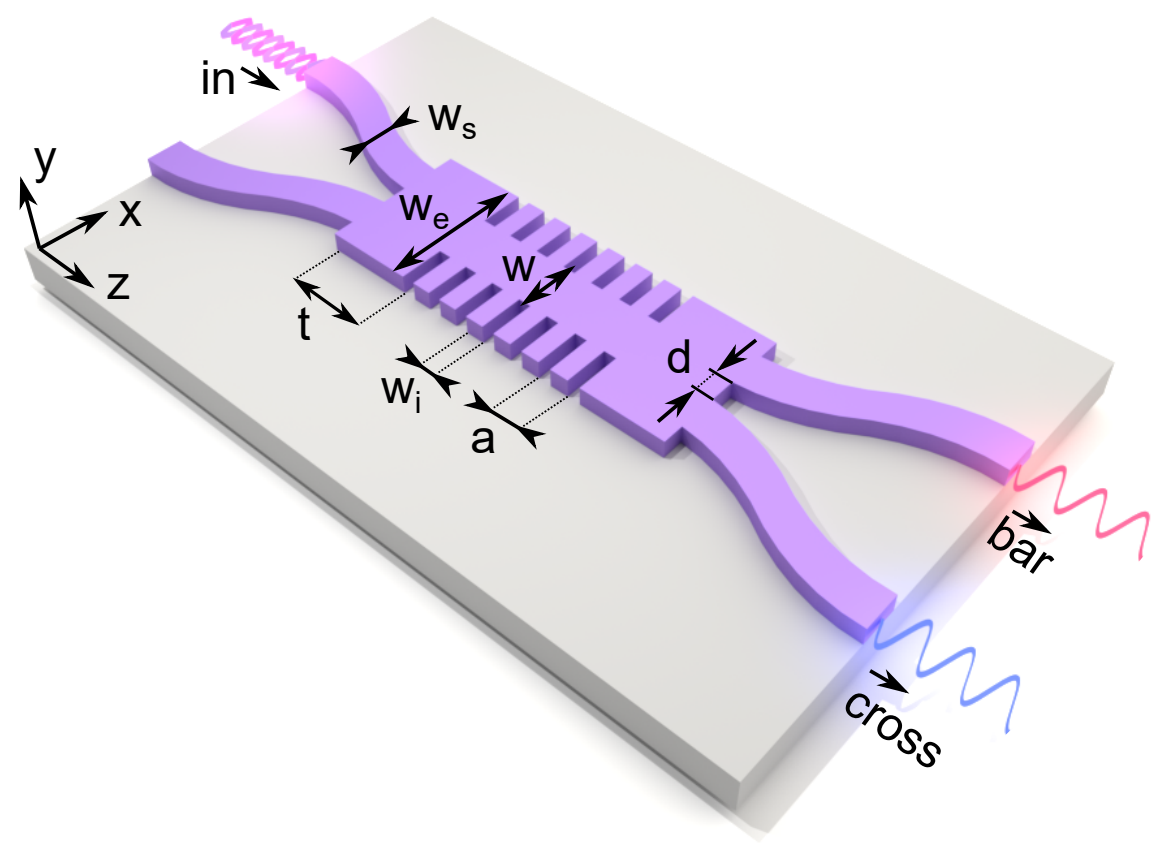

Fig. D.1. Device schematic of the proposed $2 \times 2$ optical switch design, composed of two input and output single-mode silicon waveguides, a bimodal 1D PhC structure and a rectangular taper or transition between these two parts, placed both at the input and output interfaces.

have been computed by Plane Wave Expansion (PWE) numerical methods using the MIT Photonics Bands (MPB) software [39]. As a result, a bimodal behavior between the third even band and the second odd band is obtained for an operating wavelength around 1550 $\mathrm{nm}$, as depicted by the green shaded area in Fig. 2(a). In this region, the third even and second odd bands are formed by the fundamental and the first higher order odd modes folded into the Brillouin zone. Note that the odd mode presents a slow light behavior at two different wavelengths: at the anti-crossing point with the third odd band and at the end of the first Brillouin zone, both marked with circles in Fig. 2(a) and (b). At these wavelengths, the group index of the odd mode is drastically increased whereas a highly dispersive region with a large bandwidth is obtained in between, as shown in the lower graph in Fig. 2(b). On the other hand, the even mode presents a non-dispersive behavior with low group index values for the entire bimodal region. Therefore, a relatively flat and broad spectral region exhibiting a large group index contrast between the even and odd modes is obtained in the bimodal region.

The electric field distribution of the even and odd modes at the flat region of the group index is shown in Fig. 2(c). The even mode is strongly confined within the central waveguide, while the odd mode interacts with the transversal corrugations, which causes the highly dispersive behavior of this mode. Fullyvectorial 3D Finite-Difference Time-Domain (FDTD) simulations using CST Studio software have been employed to 
(a)

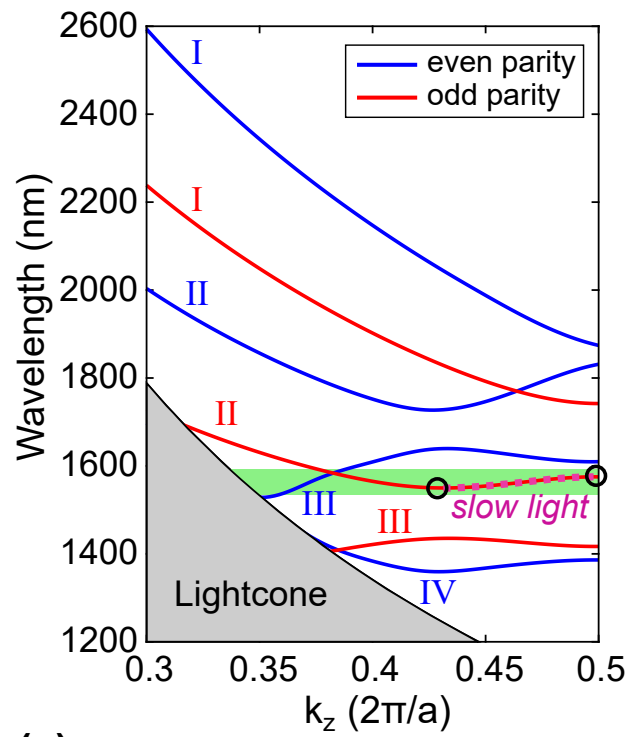

(b)

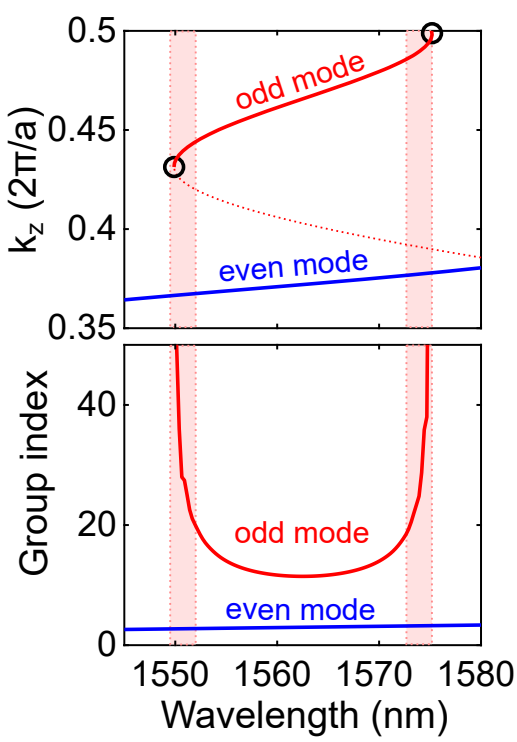

(c)
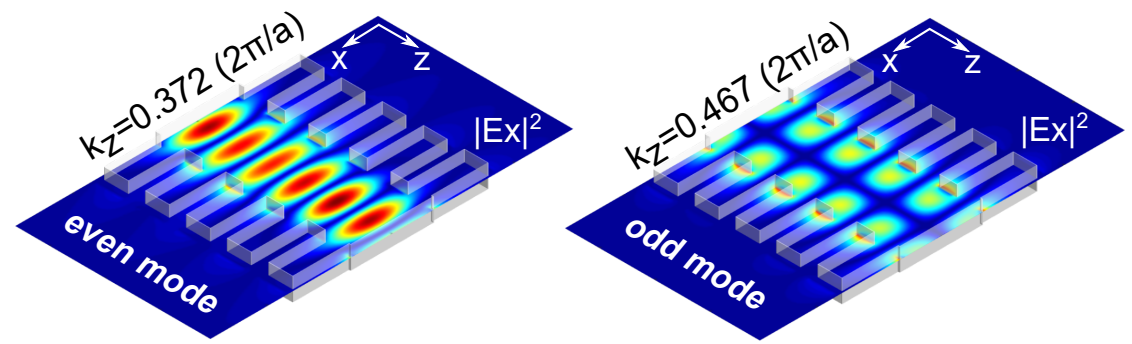

(d)
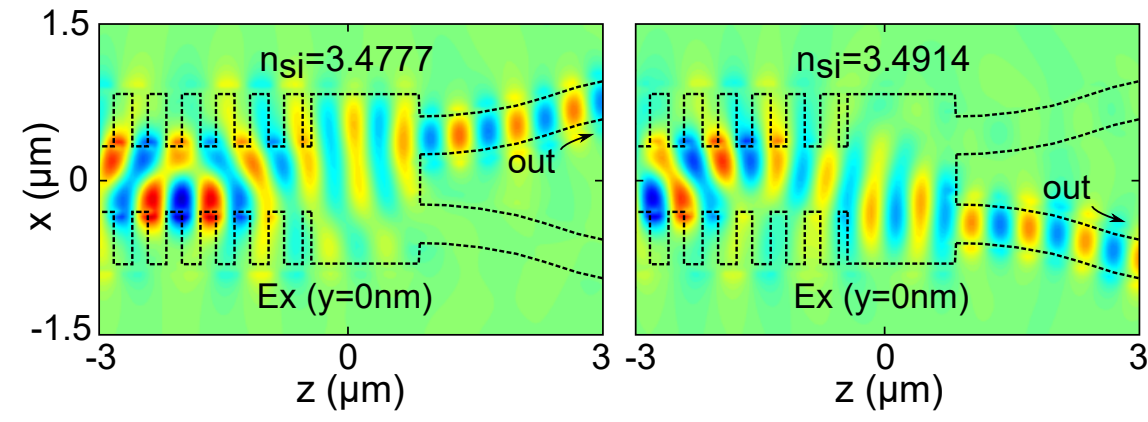

Fig. D.2. (a) Band structure of the 1D PhC periodic unit cell for the TE-like polarized bands. The dimensions considered for the simulations are $a=370 \mathrm{~nm}, w_{i}=220 \mathrm{~nm}, w_{e}=1700 \mathrm{~nm}, w=650$ $\mathrm{nm}$ and $w_{s}=450 \mathrm{~nm}$, with a height of $220 \mathrm{~nm}$. The roman numerals indicate the band formation order for the even and odd parity bands. The green shaded area indicates the bimodal region of interest, where the odd parity band becomes slow light. Circles mark the region where the group velocity of the band is critically reduced. (b) Detailed band structure and group index for both even and odd modes in the bimodal region, upper and lower graph, respectively. (c) Absolute value of the electric field $x$-component at $y=0 \mathrm{~nm}$ plane for the third even parity band and the second odd parity band, both at the bimodal region at $1564 \mathrm{~nm}$. (d) 3D-FDTD simulation of the real part $x$-component electric field propagation at $y=0 \mathrm{~nm}$ plane when the silicon refractive index changes at $1556 \mathrm{~nm}$ wavelength. 
compute the electric field propagation in the optical switch by injecting light into an input waveguide port, as depicted in Fig. 2(d). The complete device with the input and output singlemode waveguides, the tapers and the bimodal periodic structure has been considered to evaluate the switching performance when the silicon refractive index is changed. The full optical switching from the bar to the cross states can be achieved by a bimodal structure of only $N=100$ periods with a silicon refractive index change of 0.0137 .

To optimize the switching performance, the most critical design parameter that determines the effective index contrast between the even and odd modes is the transversal element length of the periodic structure, $w_{e}$. By increasing this parameter, the spectral distance between the slow light regions is reduced, thus increasing the group index in the flat region, but at expenses of reducing the optical bandwidth. This fact is due to the influence of such parameter on the cut-off frequency of the higher order odd mode. However, increasing the group index of slow light modes also leads to a reduction of the

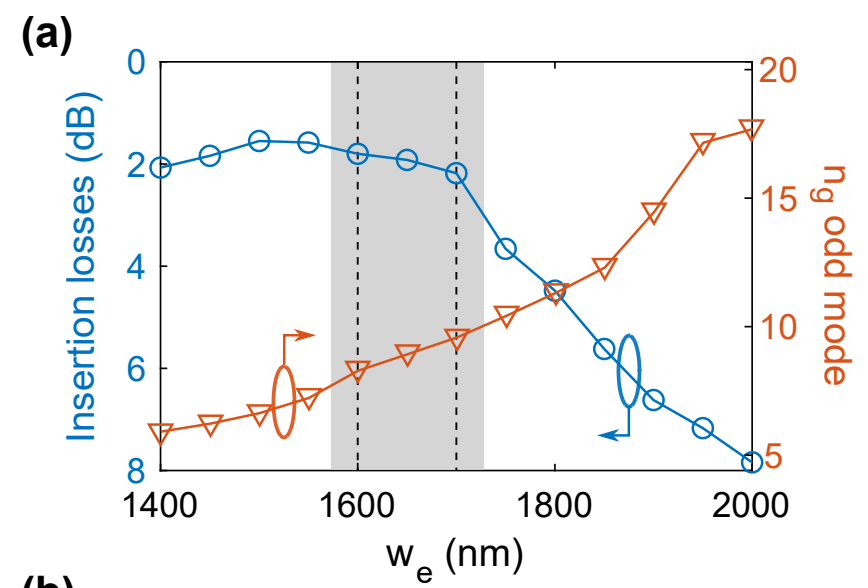

(b)

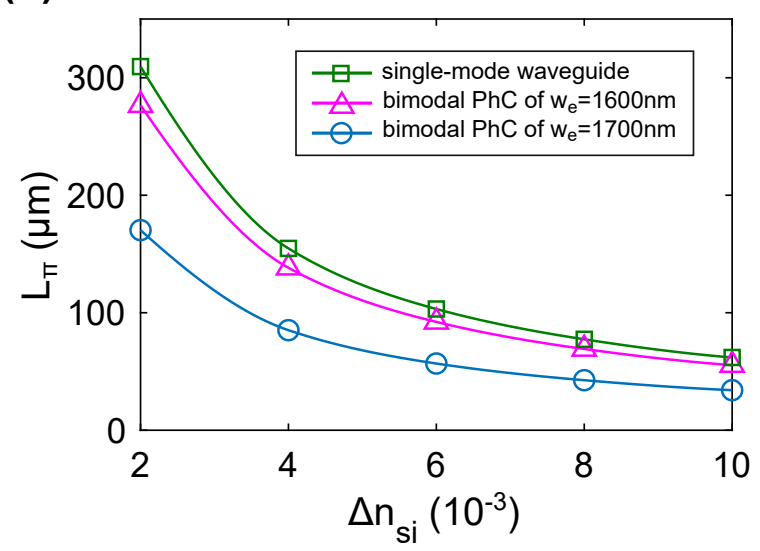

Fig. D.3. (a) Trade-off between the insertion losses and the group index of the odd parity mode as a function of the transversal element width $w_{e}$. The rest of the design parameters are the same previously considered. (b) Simulated $\pi$ length for different silicon refractive index increments at a common wavelength of $1560 \mathrm{~nm}$. 
coupling efficiency that increases the insertion losses, which must be also considered. Additionally, insertion losses are also due to the field distribution mismatch between the fundamental mode of the in-output waveguide and the higher order mode of the PhC. Figure 3(a) shows the trade-off between the group index obtained for the odd mode and the insertion losses, both in the flat region. Note that these values remain around $2 \mathrm{~dB}$ up to a $w_{e}$ of $1700 \mathrm{~nm}$, from which the design becomes highly lossy as the group index increases. Therefore, the design presents high tolerances to fabrication deviations regarding $w_{e}$. Two different designs of $w_{e 1}=1600 \mathrm{~nm}$ and $w_{e 2}=1700 \mathrm{~nm}$ with odd mode group indices of 7.85 and 9.14, respectively, have been considered for changes in the silicon refractive index. In bimodal waveguides, since both modes interact with variations in the material optical properties, the length required to obtain a phase shift of $\pi$ for a given change in refractive index is mathematically expressed as

$$
L_{\pi}=\frac{\lambda}{2\left(\Delta n_{e f f, o}-\Delta n_{e f f, e}\right)}
$$

where $\lambda$ is the wavelength, and $\Delta n_{e f f, o}$ and $\Delta n_{e f f, e}$ is the change in the effective index of the odd and even modes, respectively. Figure 3(b) depicts the $\pi$ lengths obtained for different silicon refractive index changes at a wavelength of $1560 \mathrm{~nm}$, which is within the flat region of group index. Both $w_{e 1}$ and $w_{e 2}$ designs of the bimodal 1D PhC have been computed, as well as for a conventional homogeneous singlemode waveguide of $450 \mathrm{~nm}$ width and $220 \mathrm{~nm}$ thickness. The length obtained in the $1 \mathrm{D} \mathrm{PhC}$ waveguides is shorter than in the homogeneous waveguide, even though in the bimodal case the resulting effective index change is the difference between the two modes contribution. In addition, the length obtained in the $w_{e 2}=$ design is clearly shorter than in the $w_{e 1}$ case, due to the higher group index obtained for the first configuration. As a result, compact footprints can be achieved in the $w_{e 2}$ design since the interferometry is produced in a single-channel waveguide and very short lengths are needed. Specifically, two lengths of $37 \mu \mathrm{m}$ and 74 $\mu \mathrm{m}$, corresponding to 100 and 200 periods, are considered for the experimental evaluation for the $w_{e 2}$ and $w_{e 1}$ configurations, respectively.

\subsubsection{Fabrication and characterization of the chip}

The two $w_{e 1}$ and the $w_{e 2}$ bimodal PhC designs with their respective lengths previously determined have been fabricated on a silicon-on-insulator platform with a silicon layer thickness of $220 \mathrm{~nm}$ and a silica buried layer of $2 \mu \mathrm{m}$. Regarding the fabrication process, $30 \mathrm{KeV}$ of acceleration voltage and $30 \mu \mathrm{m}$ of aperture size have been employed in the electron-beam lithography process to expose the photonic structures on a hydrogen silsesquioxane (HSQ) negative resist. Likewise, inductively plasma etching has been used to transfer the photonic designs onto the silicon layer. Finally, a silica cover layer of 
$700 \mathrm{~nm}$ has been deposited on the sample, on which a lift-off process has been used to evaporate titanium heaters of $160 \mathrm{~nm}$ thickness over the $1 \mathrm{D}$ PhC structures. The heaters dimensions are $37 \mu \mathrm{m} \times 4 \mu \mathrm{m}$ in the short $w_{e 2}$ design and $74 \mu \mathrm{m} \times 4 \mu \mathrm{m}$ in the long $w_{e 2}$ design. The dimensions of the titanium pads are $100 \mu \mathrm{m} \times 100 \mu \mathrm{m}$ in both configurations, with a separation between them of $200 \mu \mathrm{m}$.

Figure 4(a) shows the optical microscope images of the fabricated chip with both $w_{e 1}$ and $w_{e 2}$ designs, and their corresponding heaters. A scanning electron microscope (SEM) image of the entire fabricated switching structure of $N=200$ periods and $w_{e 1}$ is shown in Fig. 4(b) and a detailed image of the bimodal $1 \mathrm{D} \mathrm{PhC}$ waveguide is shown in Fig. 4(c). For the optical characterization, a continuous wave laser (Keysight 81980) with a TE polarizer was employed to inject light into the chip by using on-chip grating couplers. A synchronized power meter (Keysight $81636 \mathrm{~B}$ ) at the output, controlled by a LabVIEW application, was used to record the transmitted spectra.

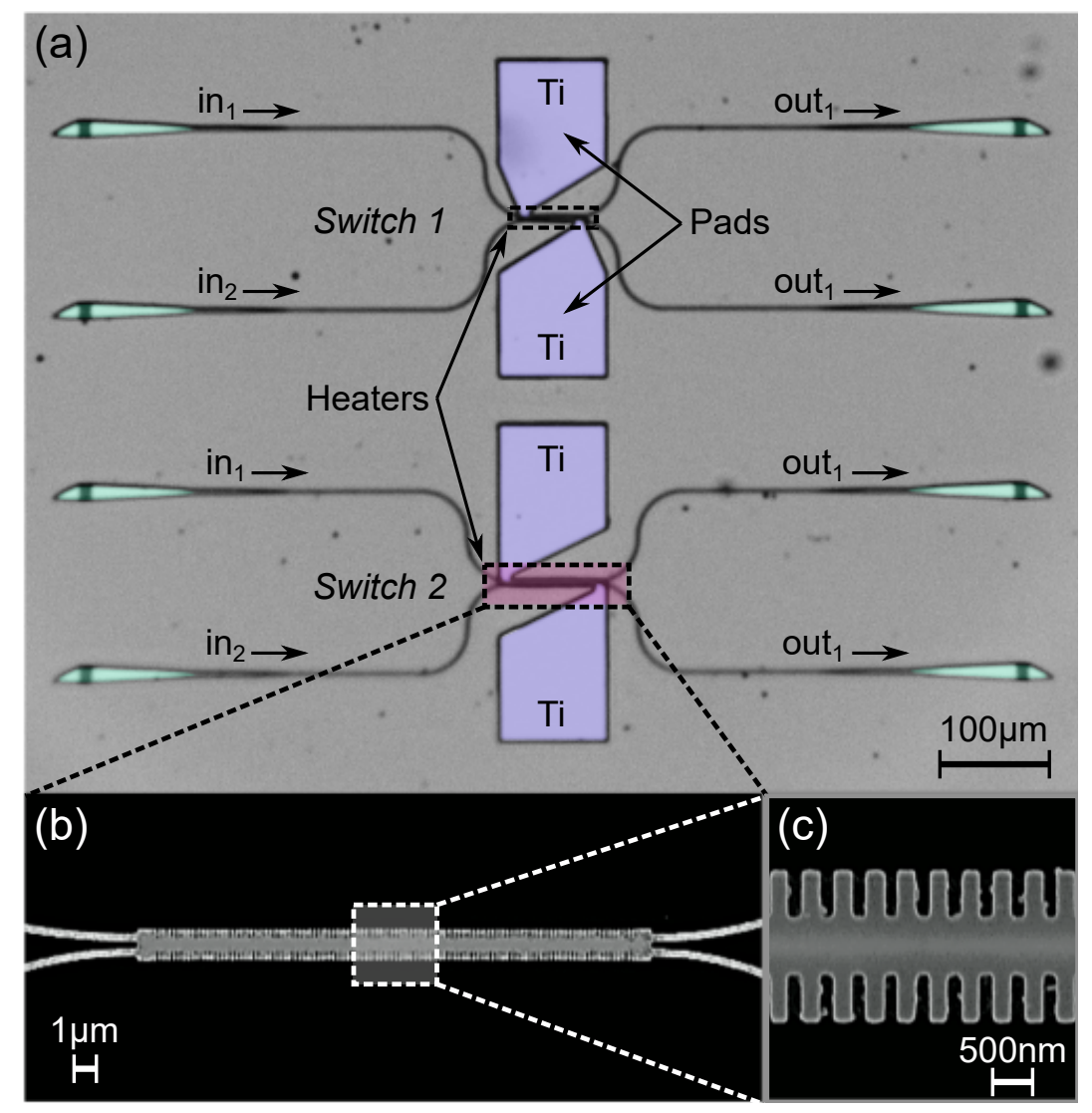

Fig. D.4. (a) Optical microscope image of the fabricated chip with the input and output grating couplers, the titanium (Ti) heaters and lateral pads. Upper and lower switches have a length of $37 \mu \mathrm{m}$ and $74 \mu \mathrm{m}$ for $w_{e 2}$ and $w_{e 1}$ designs, respectively. (b) SEM image of the optical switch of $N=200$ periods and $w_{e 2}=1600 \mathrm{~nm}$ with the input and output single mode waveguides and (c) detailed 1D PhC structure in silicon. 


\subsubsection{Experimental demonstration}

The normalized transmitted spectra for the bar and cross states are depicted for both $w_{e 1}$ and $w_{e 2}$ configurations in Fig. 5. As it is shown, an interference pattern is experimentally obtained as a result of the constructive and destructive interferences between the even and odd modes in the bimodal 1D PhC. The number of spectral peaks available in Fig. 5(a) is higher than in Fig. 5(b) due to the increment in the physical length in the first case. It should be also noted that the modal excitation is decreased for those wavelengths near the slow light regions predicted in Fig. 2(b), while the coupling efficiency is improved in between. In this region, experimental insertion losses around $1.9 \mathrm{~dB}$ and extinction ratios higher than $17 \mathrm{~dB}$ are observed for $w_{e 1}$ and $w_{e 2}$ configurations at a wavelength of 1576 $\mathrm{nm}$ and $1590 \mathrm{~nm}$, respectively, which is in a good agreement with the simulations. For these wavelengths, a crosstalk of $15 \mathrm{~dB}$ between bar and cross states is measured with a bandwidth of $0.5 \mathrm{~nm}$ and $1 \mathrm{~nm}$ for $w_{e 1}$ and $w_{e 2}$ configurations, respectively.

To determine the dispersive behavior of the bimodal 1D PhC [18], the experimental group index of the higher order odd mode is calculated from the bimodal oscillations in the transmitted spectra by using the following equation:

$$
n_{g}^{o}(\lambda)=\frac{\lambda_{\max } \lambda_{\min }}{2 L\left(\lambda_{\max }-\lambda_{\min }\right)}+n_{g}^{e}(\lambda)
$$

where $\lambda_{\max }$ is the maxima and $\lambda_{\text {min }}$ the minima positions of the transmission bimodal oscillations, $L$ the length of the bimodal region and $n_{g}^{e}$ is the simulated group index of the even mode as a function of wavelength. In Fig. 5(a) and (b) the positions of the maxima and minima are marked with circles for the bar port transmitted spectrum. The resulting experimental group index obtained is depicted in Fig. 5(c), as well as the fitting curve and the simulated even mode group index. These experimental measurements match the theoretical results in Fig. 2(b), regarding the definition of two slow light zones of extremely large group index contrast. Between these two zones, a flat region of almost a constant group index for the odd mode is obtained. A specific group index value of 7.6 and 9 at the wavelengths previously considered is experimentally obtained for the $w_{e 1}$ and $w_{e 2}$ configurations, respectively, which perfectly match the simulations in Fig. 3(a). Moreover, the optical bandwidth of the group index flat region is reduced because of the influence of the $w_{e 2}$ dimension in the band structure formation, as it was theoretically predicted. A large group index contrast flat region of $45 \mathrm{~nm}$ and $33 \mathrm{~nm}$ is experimentally measured for the $w_{e 1}$ and $w_{e 2}$ designs, respectively.

To validate the device as an optical switch, the transmitted spectra in the bar and cross states have been recorded when applying an electrical signal to the titanium heaters. Due to the thermo-optic coefficient of silicon, the optical response is tuned by the temperature 
(a)

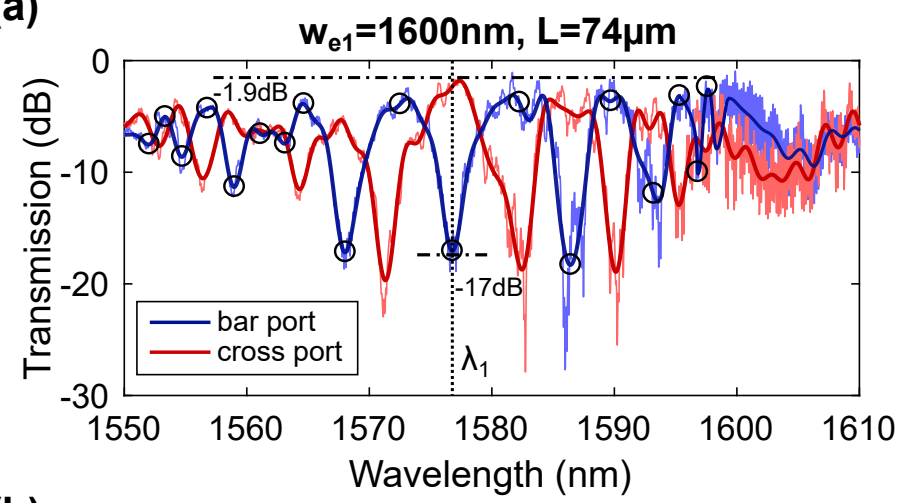

(b)

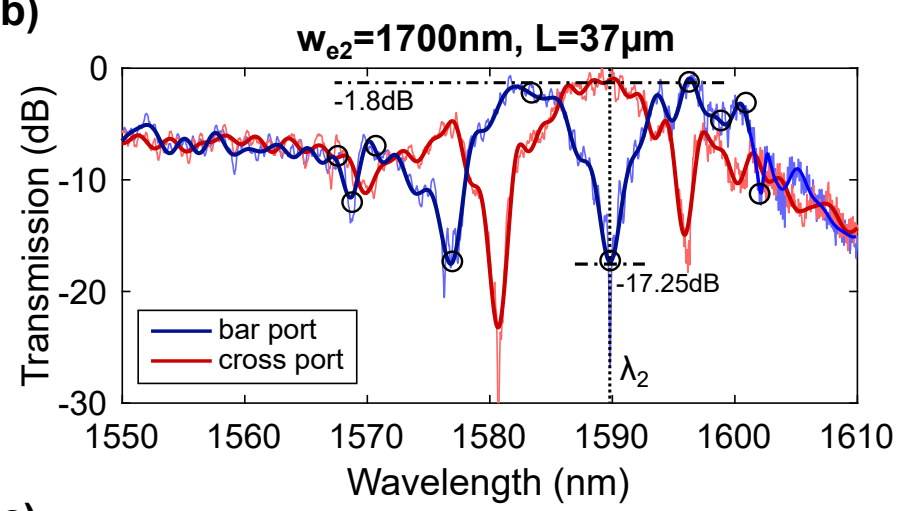

(c)

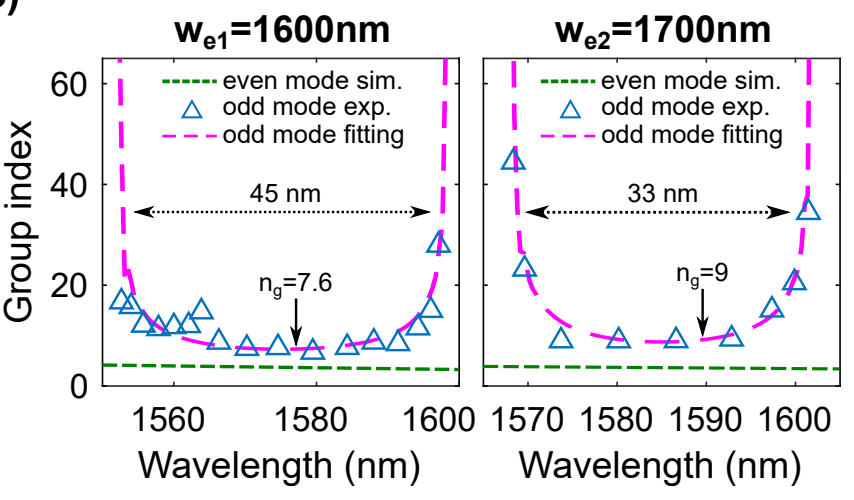

Fig. D.5. Normalized transmission spectra of the fabricated optical $2 \times 2$ switches with a transversal element width (a) $w_{e 1}$ and (b) $w_{e 2}$ with a silica cover as upper cladding. The normalization has been calculated respect to a reference waveguide of the same length. The filtered spectra are shown over the raw experimental data, in dark and light colors, respectively. Maxima and minima peaks corresponding to the constructive and destructive interferences are marked with circles. (c) Experimental group index calculated from the spectra minima and maxima peaks as a function of wavelength for both $w_{e 1}$ and $w_{e 2}$ designs.

changes in the structure caused by the heaters. Figure 6(a) and (b) shows the optical response in the bar and cross states of the $w_{e 1}$ and $w_{e 2}$ switches, respectively, for different voltages. The results present a positive spectral shift originated by an increment in the temperature, which corresponds to a phase shift around $\pi$ for both designs. To determine 
the power consumption of the switch, the spectral dips previously detailed at $1576 \mathrm{~nm}$ and $1590 \mathrm{~nm}$ have been considered in the $w_{e 1}$ and $w_{e 2}$ designs, respectively. The phase shift accumulated in the bimodal waveguide may be obtained from the transmitted spectra as:

$$
\Delta \phi=\frac{2 \Delta \lambda}{\left(F S R_{H}+F S R_{L}\right)}
$$

where $\Delta \lambda$ is the spectral shift of the transmission dip, and $F S R_{H, L}$ are the free spectral range between consecutive dips at higher and lower wavelengths, respectively. In the left graph of Fig. 6(c), the estimated phase shift with Eq. (3) is depicted as a function of the applied electrical power. As it was previously predicted, the $w_{e 2}$ design presents a higher switching efficiency than the $w_{e 1}$ design, as a result of the higher odd mode group index for this configuration. Concretely, a power consumption of $19.5 \mathrm{~mW}$ is demonstrated in the $w_{e 2}$ configuration, whereas $24.5 \mathrm{~mW}$ is obtained in the $w_{e 1}$ configuration, for a $\pi$-phase shift. The right graph of Fig. 6(c) depicts the optical power of the bar and cross states versus the applied electrical power, for both wavelengths previously considered within the flat region of the group index. It is shown that a lower electrical power is needed in the $w_{e 2}$ design to switch from a minimum optical peak to a maximum. Consequently, a higher power consumption is required in the $w_{e 1}$ design, which confirms the higher efficiency obtained for the $w_{e 2}$ configuration.

\subsubsection{Conclusion}

To conclude, we have demonstrated an ultra-compact electro-optical 2x2 switch based on a slow-light-enhanced bimodal waveguide and driven by the thermo-optic effect in silicon. In contrast to other similar work [37], the periodic unit cell is designed to support two modes of both even and odd symmetries in the bimodal part of the band structure. We benefit from this fact to engineer a broadband region of large group index contrast, thereby improving the switching performance. Two different designs have been considered to show the trade-off between the group index contrast and the insertion losses. In comparison with the literature, simulations have demonstrated a higher efficiency for both slow-lightenhanced bimodal configurations compared to a standard silicon waveguide integrated in ring resonators and MZI-based switches [3], [4], [6]. Moreover, the interferometry has been carried out in a single-channel structure without the need of additional photonic elements, which have significantly reduced the final size of the device. As a result, an ultra-compact switch with a footprint of only $63 \mu \mathrm{m} 2$ was achieved, which is smaller than the ones reported in related PhC-based works in MZI configurations [17], [21] and similar to the ones observed in 2D PhC [22]-[25], but in our case in a straightforward design that eases the fabrication processes and cost for high-level production. An insertion loss of $1.8 \mathrm{~dB}$ and a crosstalk of 15 
(a)

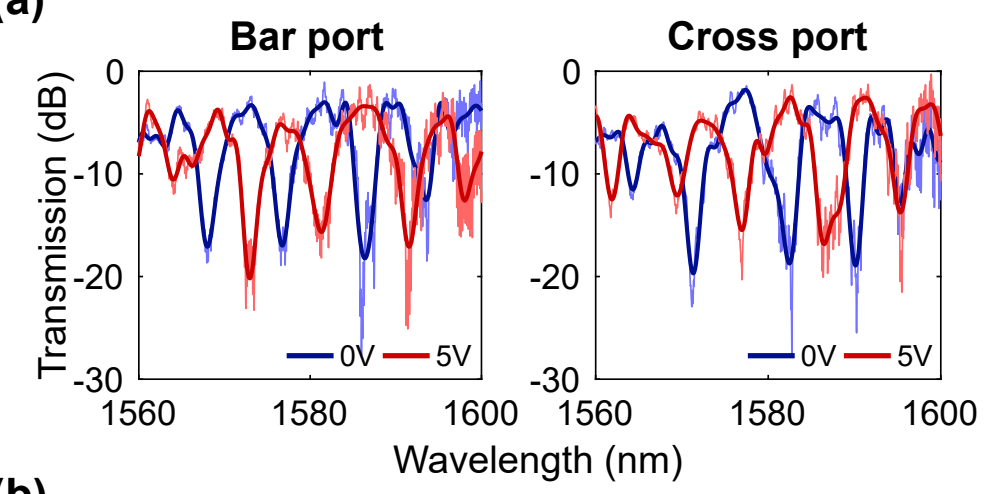

(b)

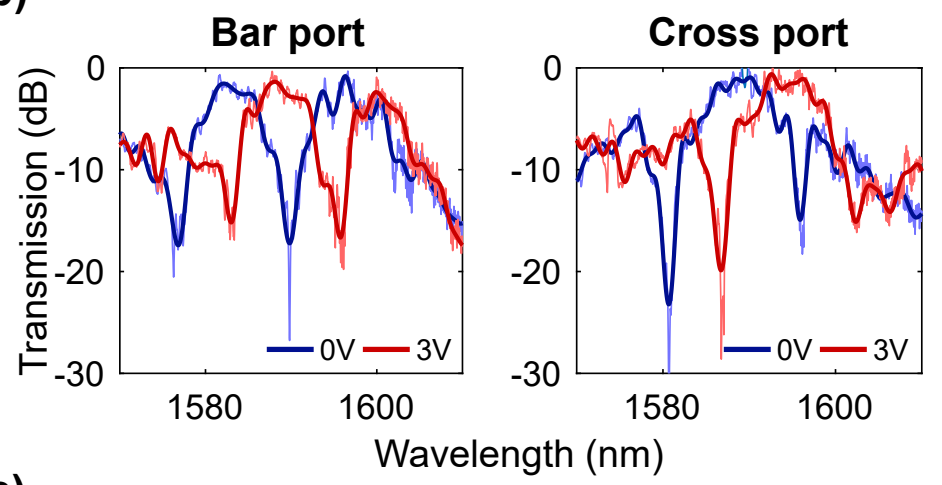

(c)
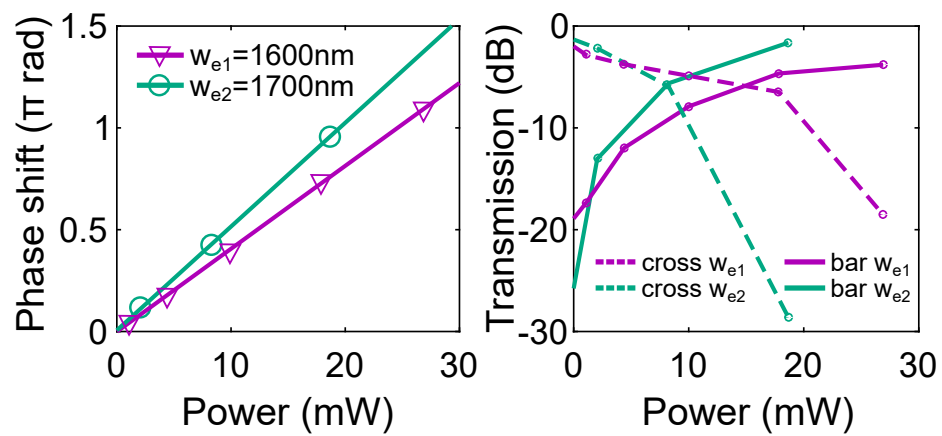

Fig. D.6. Normalized transmission spectra for different voltages and for both designs of (a) $w_{e 1}=$ $1600 \mathrm{~nm}$ with a device length of $74 \mu \mathrm{m}$ and (b) $w_{e 1}=1700 \mathrm{~nm}$ with a device length of $37 \mu \mathrm{m}$. (c) Phase shift and optical power as a function of the electrical power applied, left and right graph, respectively. A wavelength of $1576 \mathrm{~nm}$ and $1590 \mathrm{~nm}$ have been considered for the $w_{e 1}$ and $w_{e 2}$ designs, respectively, both in the flat region of the group index.

$\mathrm{dB}$ over a $1 \mathrm{~nm}$ bandwidth were obtained with a power consumption of $19.5 \mathrm{~mW}$. The resulting bandwidth is smaller than for conventional MZI switches [7] but higher than in the case of RR switches [5]. On the other hand, insertion losses is higher than in conventional MZIs or ring resonators, where values below $0.5 \mathrm{~dB}$ have been reported by using thermo-optic phase shifters [40], [41]. However, the taper geometry could be further optimized to minimize insertion losses [42], while power consumption could also 
be reduced with an improved design of the heaters [11]. The results suggest the use of these types of structures in photonic switching for datacenters in which large matrices are not required and the overall photonic integrated circuit size would be drastically reduced [43]. Furthermore, future work in lowering losses would be highly desirable for improving scalability and addressing other potential applications such as LIDAR systems, programmable circuits, or neuromorphic computing.

\section{Acknowledgements}

This work was supported by Generalitat Valenciana under AVANTI/2019/123 and ACIF/2019/009 grants; by the Spanish Ministerio de Ciencia e Innovación through PID2019-106965RB-C21 and PID2019- 111460GB-I00 projects, and by the European Union through the operational program of the European Regional Development Fund (FEDER) of the Valencia Regional Government 2014-2020.

\subsubsection{References}

1. D. Thomson et al., "Roadmap on silicon photonics,” J. Opt., vol. 18, no. 7, 2016.

2. H. Subbaraman et al., "Recent advances in silicon-based passive and active optical interconnects," Opt. Express, vol. 23, no. 3, p. 2487, 2015.

3. D. Nikolova et al., "Scaling silicon photonic switch fabrics for data center interconnection networks," Opt. Express, vol. 23, no. 2, p. 1159, 2015.

4. P. Dong, S. F. Preble, and M. Lipson, "All-optical compact silicon comb switch," Opt. Express, vol. 15, no. 15, p. 9600, 2007.

5. A. Biberman et al., "Broadband silicon photonic electrooptic switch for photonic interconnection networks," IEEE Photonics Technol. Lett., vol. 23, no. 8, pp. 504-506, 2011.

6. A. Densmore et al., "Compact and low power thermo-optic switch using folded silicon waveguides,” Opt. Express, vol. 17, no. 13, p. 10457, 2009.

7. J. Van Campenhout, W. M. Green, S. Assefa, and Y. A. Vlasov, "Low-power, $2 \times 2$ silicon electro-optic switch with 110-nm bandwidth for broadband reconfigurable optical networks," Opt. Express, vol. 17, no. 26, p. 24020, 2009.

8. M. R. Watts, J. Sun, C. Derose, D. C. Trotter, R. W. Young, and G. N. Nielson, "Adiabatic thermo-optic Mach - Zehnder switch,” Opt. Lett., vol. 38, no. 5, pp. 733-735, 2013. 
9. F. Wang, J. Yang, L. Chen, X. Jiang, and M. Wang, "Optical switch based on multimode interference coupler,” IEEE Photonics Technol. Lett., vol. 18, no. 2, pp. 421-423, 2006.

10. Á. Rosa, A. Gutiérrez, A. Brimont, A. Griol, and P. Sanchis, "High performace silicon 2x2 optical switch based on a thermo-optically tunable multimode interference coupler and efficient electrodes," Opt. Express, vol. 24, no. 1, p. 191, 2016.

11. J. Parra, J. Hurtado, A. Griol, and P. Sanchis, "Ultra-low loss hybrid ITO/Si thermo-optic phase shifter with optimized power consumption," Opt. Express, vol. 28, no. 7, p. 9393, 2020 .

12. R. Amin et al., "0.52 v mm ITO-based Mach-Zehnder modulator in silicon photonics," APL Photonics, vol. 3, no. 12, pp. 0-11, 2018.

13. E. Li, B. A. Nia, B. Zhou, and A. X. Wang, "Transparent conductive oxide-gated silicon microring with extreme resonance wavelength tunability,” Photonics Res., vol. 7, no. 4, p. 473, 2019.

14. R. Amin et al., "Sub-wavelength GHz-fast broadband ITO Mach-Zehnder modulator on silicon photonics," Optica, vol. 7, no. 4, p. 333, 2020.

15. S. Campione et al., "Submicrometer Epsilon-Near-Zero Electroabsorption Modulators Enabled by High-Mobility Cadmium Oxide,” IEEE Photonics J., vol. 9, no. 4, 2017.

16. J. Parra, I. Olivares, F. Ramos, and P. Sanchis, "Ultra-compact nonvolatile Mach-Zehnder switch enabled by a high-mobility transparent conducting oxide," Opt. Lett., vol. 45, no. 6, p. 1503, 2020.

17. E. A. Camargo, H. M. H. Chong, and R. M. De La Rue, "2D Photonic crystal thermo-optic switch based on AlGaAs/GaAs epitaxial structure," Opt. Express, vol. 12, no. 4 , p. $588,2004$.

18. Y. A. Vlasov, M. O’Boyle, H. F. Hamann, and S. J. McNab, "Active control of slow light on a chip with photonic crystal waveguides,” Nature, vol. 438, no. 7064, pp. 65-69, 2005.

19. T. F. Krauss, "Slow light in photonic crystal waveguides," J. Phys. D. Appl. Phys., vol. 40, no. 9, pp. 2666-2670, 2007.

20. T. Baba, "Slow light in photonic crystals," Nat. Photonics, vol. 2, no. 8, pp. 465-473, 2008.

21. H. Nakamura, Y. Sugimoto, K. Kanamoto, and N. Ikeda, "Ultra-fast photonic crystal/quantum dot all-optical switch for future photonic networks," Opt. Express, vol. 12, no. 26, pp. 6606-6614, 2004. 
22. Z. Li, Y. Zhang, and B. Li, "Terahertz photonic crystal switch in silicon based on self-imaging principle," Opt. Express, vol. 14, no. 9, p. 3887, 2006.

23. N. Yamamoto, T. Ogawa, and K. Komori, "Photonic crystal directional coupler switch with small switching length and wide bandwidth," Opt. Express, vol. 14, no. 3, p. 1223, 2006.

24. D. M. Beggs, T. P. White, L. O'Faolain, and T. F. Krauss, "Ultracompact and low-power optical switch based on silicon photonic crystals," Opt. Lett., vol. 33, no. 2, p. 147, 2008.

25. K. Nozaki et al., "Sub-femtojoule all-optical switching using a photonic-crystal nanocavity," Nat. Photonics, vol. 4, no. 7, pp. 477-483, 2010.

26. Y. A. Vlasov and S. J. McNab, "Coupling into the slow light mode in slab-type photonic crystal waveguides,” Opt. Lett., vol. 31, no. 1, p. 50, 2006.

27. M. Notomi, "Manipulating light with strongly modulated photonic crystals," Reports Prog. Phys., vol. 73, no. 9, 2010.

28. M. Centini et al., "Dispersive properties of finite, one-dimensional photonic band gap structures: Applications to nonlinear quadratic interactions," Phys. Rev. E, vol. 60, no. 4, pp. 4891-4898, 1999.

29. M. Scalora et al., "Ultrashort pulse propagation at the photonic band edge: Large tunable group delay with minimal distortion and loss," Phys. Rev. E, vol. 54, no. 2, pp. 1078-1081, 1996.

30. W. C. L. Hopman et al., "Quasi-one-dimensional photonic crystal as a compact building-block for refractometric optical sensors," IEEE J. Sel. Top. Quantum Electron., vol. 11, no. 1, pp. 11-15, 2005.

31. R. B. Hwang, "Negative group velocity and anomalous transmission in a one-dimensionally periodic waveguide," IEEE Trans. Antennas Propag., vol. 54, no. 2, pp. 755-760, 2006.

32. A. Brimont, A. Griol, J. Martí, P. Sanchis, J. Martí, and P. Sanchis, "Group index engineering in silicon corrugated waveguides," IEEE Int. Conf. Gr. IV Photonics GFP, vol. 35, no. 16, pp. 39-41, 2010.

33. J. Sabek et al., "Experimental study of an evanescent-field biosensor based on 1D photonic bandgap structures," Beilstein J. Nanotechnol., vol. 10, pp. 967-974, 2019.

34. A. Brimont et al., "High speed silicon electro-optical modulators enhanced via slow light propagation," Opt. Express, vol. 19, no. 21, p. 20876, 2011. 
35. L. Torrijos-Morán and J. García-Rupérez, "Single-channel bimodal interferometric sensor using subwavelength structures," Opt. Express, vol. 27, no. 6, pp. 8168-8179, 2019.

36. L. Torrijos-Morán, A. Griol, and J. García-Rupérez, "Experimental study of subwavelength grating bimodal waveguides as ultrasensitive interferometric sensors," Opt. Lett., vol. 44, no. 1, pp. 4702-4705, 2019.

37. L. Torrijos-Morán, A. Griol, and J. García-Rupérez, "Slow light bimodal interferometry in one- dimensional photonic crystal waveguides," Light Sci. Appl., vol. 10, no. 16, 2021.

38. M. Notomi, K. Yamada, A. Shinya, J. Takahashi, C. Takahashi, and I. Yokohama, "Extremely large group-velocity dispersion of linedefect waveguides in photonic crystal slabs," Phys. Rev. Lett., vol. 87, no. 25, pp. 253902-253902-4, 2001.

39. S. Johnson and J. Joannopoulos, "Block-iterative frequency-domain methods for Maxwell's equations in a planewave basis," Opt. Express, vol. 8, no. 3, pp. 173-190, 2001.

40. Q. Fang et al., "Ultralow power silicon photonics thermo-optic switch with suspended phase arms,” IEEE Photonics Technol. Lett., vol. 23, no. 8, pp. 525-527, 2011.

41. N. Sherwood-Droz et al., "Optical $4 \times 4$ hitless Silicon router for optical Networks-on-Chip (NoC): erratum,” Opt. Express, vol. 16, no. 23, p. 19395, 2008.

42. X. Zhao, H. Dalir, X. Xu, and R. T. Chen, "Efficient coupling into slow-light one-dimensional fishbone waveguide by mode converter method," Appl. Phys. Express, vol. 10, no. 7, 2017.

43. Q. Cheng, S. Rumley, M. Bahadori, and K. Bergman, "Photonic switching in high performance datacenters [Invited]," Opt. Express, vol. 26, no. 12, p. 16022, 2018. 


\subsection{Paper E:}

Optics Express 29, 15 (2021)

\section{Design of slow-light-enhanced bimodal interferometers using dimensionality reduction techniques}

Luis Torrijos-Morán and Jaime García-Rupérez 



\title{
Design of slow-light-enhanced bimodal interferometers using dimensionality reduction techniques
}

\author{
Luis Torrijos-Morán, and Jaime García-Rupérez
}

\begin{abstract}
Nanophotonics Technology Center, Universitat Politècnica de València, Camino de Vera s/n, 46022 Valencia, Spain
\end{abstract}

\begin{abstract}
Interferometers usually require long paths for the ever-increasing requirements of high-performance operation, which hinders the miniaturization and integration of photonic circuits into very compact devices. Slow-light based interferometers provide interesting advantages in terms of both compactness and sensitivity, although their optimization is computationally costly and inefficient, due to the large number of parameters to be simultaneously designed. Here we propose the design of slow-light-enhanced bimodal interferometers by using principal component analysis to reduce the high-dimensional design space. A low-dimensional hyperplane containing all optimized designs is provided and investigated for changes in the silicon core and cladding refractive index. As a result, all-dielectric single-channel interferometers as modulators of only $33 \mu \mathrm{m}^{2}$ footprint and sensors with $19.2 \times 10^{3} 2 \pi \mathrm{rad} / \mathrm{RIU} \cdot \mathrm{cm}$ sensitivity values are reported and validated by two different simulation methods. This work allows the design and optimization of slow light interferometers for different applications by considering several performance criteria, which can be extended to other photonic structures.
\end{abstract}

\subsubsection{Introduction}

Optical interferometers are essential components in today integrated photonics. Among others, they are widely used in modulators, switches, sensors and programmable photonic circuits [1-4], due to their ability of converting a change in the refractive index (RI) into a shift of the relative phase between two propagating modes. To enhance the phase shift, further optimization either in the material or in the geometry is required to maximize light-matter interaction. In sensing interferometers, a strong field interaction with the cladding is desired to increase the sensitivity [5], for instance by employing plasmonic configurations [6-9] or lower RI contrast materials such as silicon nitride [10]. In contrast, typical electro-optical modulators based on Mach-Zehnder (MZI) interferometers in silicon-on-insulator (SOI) platforms require from highly confined modes within the waveguide core to assure a complete overlap of the optical and the 
electric field [11-13]. Similarly, SOI thermo-optic switches are also designed to support highly confined optical modes to optimize the interaction of the optical field with the core RI changes due to the thermo-optic coefficient of silicon [14-16]. Concurrently, interferometers performance can also be improved by engineering new structures and including them in one of the MZI arms, as in the case of slot waveguides $[17,18]$ or subwavelength grating (SWG) structures [19,20], although roughness scattering losses must be considered compared to strip waveguides [21].

On the other hand, the physical path of the interferometer limits the size of the device as long interferometric structures are typically designed to provide high-performance operation, which hinders the fabrication of densely integrated optical circuits [22]. To solve this issue, long MZIs are proposed as compact interferometers by including low-loss compact bends with small radius in the MZI arms [23], as well as bimodal (BiM) waveguide sensors where the interferometry is carried out in a single-channel structure [24]. More sophisticated BiM waveguides based on SWG structures have also been reported as compact and high-sensitivity sensors without the need of additional photonic structures $[25,26]$. Another interesting approach is to increase the optical path of the interferometer using slow light structures [27,28], while maintaining a reduced physical length. This is the case of photonic crystals (PhCs), either combining them in the arms of a MZI to develop modulators [29-31] and sensors [32], or by fully integrating an interferometric scheme in a $2 \mathrm{D}$ hole-patterned $\mathrm{PhC}$ for switching purposes $[33,34]$. In this scenario, further improvement was made by including a bimodal behavior in a single-channel slow light waveguide [35,36], demonstrating high-performance interferometers as modulators, switches, and sensors, with extremely reduced footprints compared to the abovementioned approaches. However, the optimization of these types of periodic structures is not straightforward because of the multiple design dimensions to consider. Several optimization methods based on genetic algorithms [37,38], gradient-based optimizations [39,40] or particle swarm [41,42] are proposed as useful tools to design high-performance grating couplers, SWG structures or PhCs, among others. More recently, machine learning techniques have also been demonstrated for designing optimized nanophotonic components using artificial networks $[43,44]$. Nevertheless, all these optimization methods focus on optimizing a single criterion, which makes it very difficult to obtain a general perspective of the overall device performance. In contrast, dimensionality reduction techniques offer interesting design tools to optimize multi-parameter structures by taking into consideration different performance criteria [45-48], which eases the design process for the desired application.

In this work, we propose the design and optimization of multi-variable slow-light-enhanced bimodal interferometers by using principal component analysis (PCA). A figure of merit (FoM) is provided, which allows us to characterize the desired 
bimodal band structure and extrapolate it to other different PhC designs. By using PCA of the FoM, we explore and optimize the low-dimensional design space for changes in both the silicon core and the cladding RI. Three different interferometers are presented and optimized as sensors and modulators, offering significant improvements both in sensitivity and compactness compared to other existing configurations.

\subsubsection{Figure of merit definition}

In a PhC, when two bands of the same polarization and parity intersect, they repeal each other and form an anti-crossing point where electromagnetic wave propagation is forbidden [49]. Furthermore, it has already been demonstrated how slow light bimodal interferometers, based on two modes with a large group index difference, can be designed in the vicinity of the anti-crossing point [35]. Figure la schematically shows the first three bands of a band structure in a PhC formed by the fundamental mode folded into the first Brillouin zone and the higher order mode. Figure $1 \mathrm{~b}$ and $\mathrm{c}$ depict the third bimodal band and its corresponding group index for both modes as a function of wavelength. The group index is defined as $n_{g}=c / v_{g}$ where $c$ is the speed of light in the vacuum and $v_{g}$ the group velocity, which is related to the slope of the wavelength versus the wavevector. Note that the maximum group index difference is obtained at $\lambda_{1}$, where the higher order mode reaches the end of the first Brillouin zone and becomes slow light. Conversely, at $\lambda_{2}$, both modes present a slow light behavior and almost no group index difference is observed. This operating principle, involving higher order bands, is obtained within the PhC region, while in the SWG regime only first order bands are considered. In this work, we define a FoM to characterize the curvature of the third bimodal band in order to quantify the group index difference and the bandwidth of the bimodal region. The FoM is mathematically expressed as

$$
F o M=\int_{\lambda_{1}}^{\lambda_{2}}\left(n_{g 2}-n_{g 1}\right) d \lambda
$$

where $\lambda_{1,2}$ are the entire wavelength limits of the bimodal region, and $n_{g 2,1}$ are the group index of the higher order and fundamental mode, respectively. Therefore, the FoM may be defined as the area under the curve of group index difference as a function of wavelength. By optimizing this variable, we assure a bimodal region with large group index contrast and bandwidth, which are the main parameters in order to maximize the optical path of the interferometer. The higher the group index difference, the larger the phase shift obtained for a given change in the RI, regardless the physical length. Moreover, the FoM provides information about the bimodal band curvature, which is common to other PhC designs in which an anti-crossing point is formed, see Fig. 1a. This fact will allow us to 
a

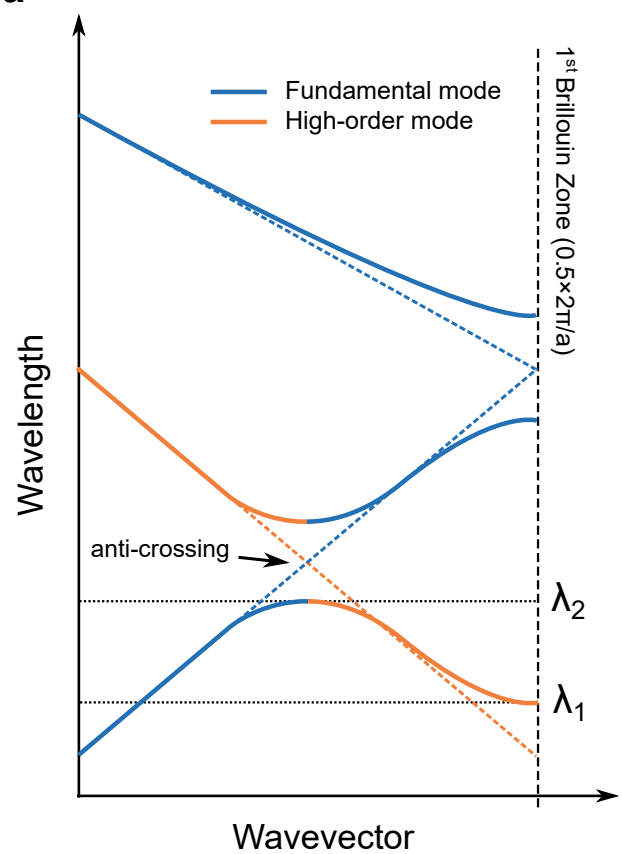

b

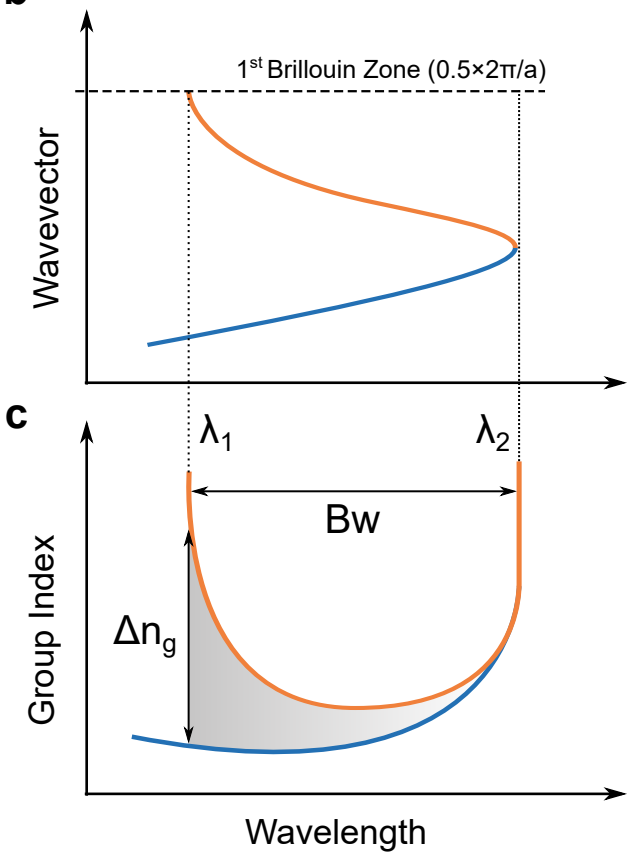

Fig. E.1. Bimodal band structure representation. a) The first three bands are shown as well as the contribution of the fundamental and high-order modes. The anti-crossing point of a $\mathrm{PhC}$ is also depicted in the intersection between the second and third band. Dashed lines represent the hypothetical band structure of a homogeneous waveguide without periodicity. b) Third band considered to perform the interferometry and c) group index as a function of wavelength. The gray shaded area represents the FoM.

compare between $\mathrm{PhC}$ configurations of the same design, and between different designs with new geometric shapes, and thus, with a different light-matter interaction.

\subsubsection{Design optimization}

In the following section, three designs based on one-dimensional $\mathrm{PhC}$ waveguides made of silicon over silica cladding are investigated for sensing and modulating purposes. The unit cell geometry is engineered to obtain a bimodal band structure with a similar curvature than the third band presented in Fig. 1b, so that it can be quantified by the FoM previously defined. To this end, a first exploration of the multi-parameter design space is carried out by using MIT photonics band (MPB) free software [50]. MPB computes definite-frequency eigenstates of Maxwell's equations by using plain wave expansion numerical methods, to obtain the band structure of the PhC. The first three bands for the transverse electric (TE)-like polarization have been computed. An initial low-resolution sweep of $n^{D}$ simulations is carried out, where $n$ is the number of values for each dimension and $D$ the number of design dimensions. A collection of good designs 
providing values of FoM above a certain threshold is selected from this initial sweep. These designs are subsequently used in the PCA to extract the sub-dimensional design space [46]. PCA finds among the multi-dimensional design space a set of orthogonal vectors that maximizes the variance of the FoM. Specifically, a two-dimensional hyperplane formed by the first two principal components is selected, which critically reduces the design space dimensionality and enables a low-computational exhaustive mapping. Thereby, a design $k$ with dimensions $\mathbf{L}_{k}=\left[L_{1, k} \ldots L_{D, k}\right]$ can be expressed as

$$
\mathbf{L}_{k}=\alpha_{k} \mathbf{V}_{1 \alpha \beta}+\beta_{k} \mathbf{V}_{2 \alpha \beta}+\mathbf{C}_{\alpha \beta}
$$

where $\alpha, \beta$ are the coefficients that describe any design in the hyperplane formed by the first two principal components vectors $\mathbf{V}_{1,2}$, and $\mathbf{C}_{\alpha \beta}$ is the origin constant vector. High-resolution sweeps of the resulting 2D hyperplane are now reachable, with a wide collection of good designs for different configurations. Besides, an exhaustive mapping of the hyperplane can be further evaluated for different criteria beyond the FoM. More precisely, we are going to investigate the interferometer as sensors and modulators, thus for changes in the cladding and silicon RI, respectively, and also for fabrication robustness.

The first 1D PhC design considered is shown in Fig. 2a, based on a periodic corrugated waveguide in the propagation direction. This design has already been investigated in Ref. [35], but here we intend to optimize it by using PCA. The unit cell dimensions are shown on the right-side part of Fig. 2a, for the periodicity $a$, central waveguide width $w$, transversal element length $w_{e}$, width $w_{i}$ and height $h$. Note that a rectangular taper for the efficient bimodal excitation is placed between the single mode in-output waveguide of width $w_{s}$ and the periodic bimodal structure, although these parameters do not affect the band calculation. The height is set to $220 \mathrm{~nm}$, which is a typical value used in SOI wafers for silicon photonics, although this parameter could be also included in the PCA optimization. As a result, a four-dimensional design of the unit cell is obtained $\left(a, w, w_{e}, w_{i}\right)$. To reduce the computational resources, the number of values for each dimension $n$ is set to a low value of 4 . Thus, an initial sweep of $4^{4}=256$ simulations has been computed to obtain the band diagram in MPB, and its respective FoM, of the entire multi-dimensional space. An initial collection of 44 good designs with a FoM over a threshold of 270 has been selected for the PCA. This value has been selected to obtain a relatively large number of designs in the initial collection.

Figure $2 \mathrm{~b}$ depicts the exhaustive mapping, as a function of $\alpha$ and $\beta$ coefficients, of the $2 \mathrm{D}$ hyperplane after the PCA with a high-resolution sweep of $30 \times 30 \mathrm{MPB}$ simulations. The black solid contour represents those designs with a FoM of 250, which is the value calculated in Ref. [35]. Those FoM values out of the color bar range are not represented 

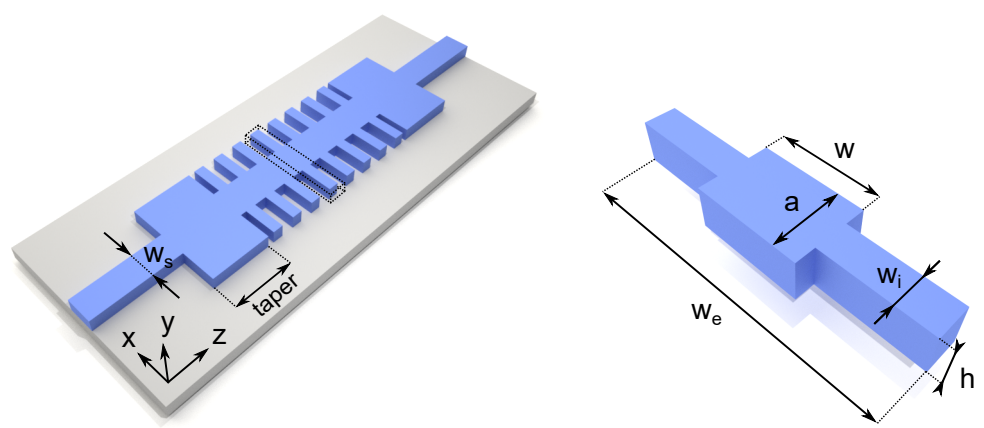

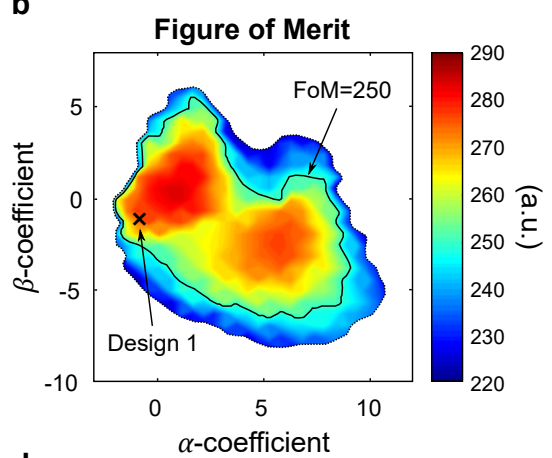

d

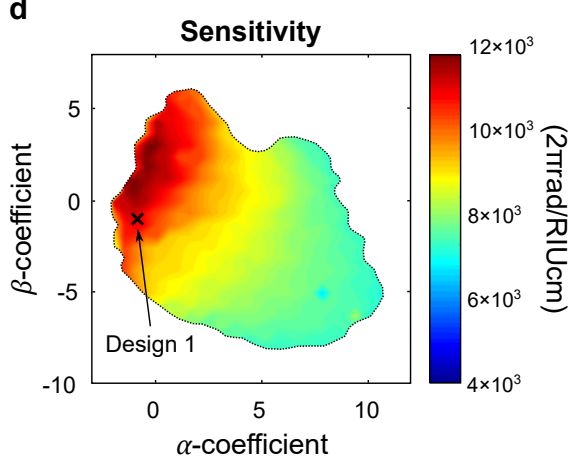

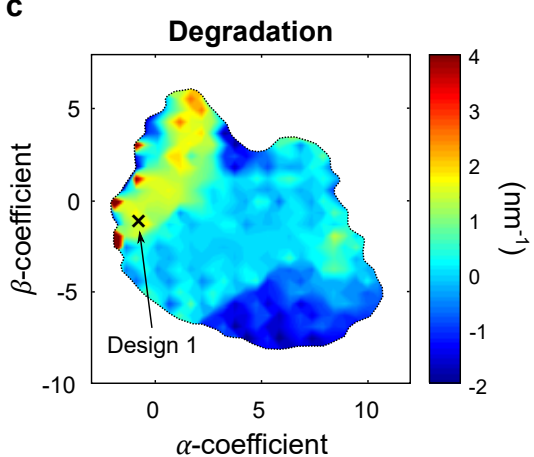

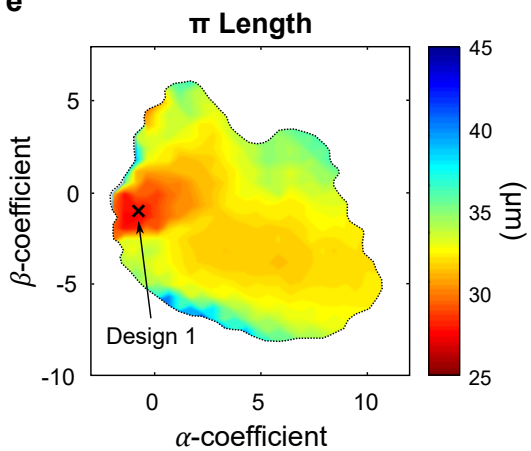

Fig. E.2. Corrugated waveguide design optimization. (a) Sketch of the complete slowlight-enhanced interferometer based on a bimodal PhC waveguide, and two single-mode waveguides at the input and output. The unit cell dimensions are also specified on the right-hand side of the figure. (b) Colormap of the figure of merit PCA, for the first and second principal components of the collection of good designs. (c) Degradation of the $\mathrm{F}$ igure of merit for a width variation of $+-10 \mathrm{~nm}$ in all the specified dimensions except for the period. (d) Upper cladding RI sensitivity normalized to a $1 \mathrm{~cm}$ length at $\lambda_{0}$ wavelength. (e) Length required for a $\pi$ phase shift at $\lambda_{0}$ wavelength and a given silicon $\mathrm{RI}$ increment of 0.01 .

to emphasize the optimized values. A relatively large region of different designs with optimized FoM values is obtained, within which two optimized FoM areas with values around 280 are observed. Likewise, the hyperplane of optimized designs is explored for small fabrications deviations of $+-10 \mathrm{~nm}$ in the design dimensions. The degradation of the FoM is shown in Fig. 2c, calculated as $\left.d=-\left(F^{+}+F^{-}-2 F_{0}\right) /\left(2 \Delta \delta_{w}\right)\right)$ where $F^{+,-}$is the FoM for a positive and negative deviation, respectively, $F_{0}$ the initial FoM without 
deviations and $\Delta \delta_{w}$ is the absolute deviation width considered. Note that a positive degradation means a reduction of the FoM when deviations occur, whereas a negative degradation will be translated into an increment of the FoM.

Figure $2 \mathrm{~d}$ and e show the colormap for the sensitivity and $\pi$ length, which are strongly related with the light-matter interaction of the propagating modes with the cladding and silicon core, respectively. The phase shift has been calculated for a given change in the cladding and in the silicon RI. Due to the dispersion of these interferometers, the phase shift strongly depends on the operating wavelength, being much higher for those regions near $\lambda_{1}$ in Fig. 1a. However, coupling losses are also incremented within the slow light region, so that a trade-off between sensitivity and losses is obtained [51]. Due to this fact, the phase shift is calculated at $\lambda_{0}$ wavelength where the group index of the higher order mode is limited to 20 , since this value has been experimentally demonstrated in similar structures [35]. At this wavelength, the sensitivity to cladding RI changes shown in Fig. $2 \mathrm{~d}$ and normalized to a length of $1 \mathrm{~cm}$, has been calculated as $S=\Delta \phi / \Delta n_{c}$, where $\Delta \phi$ is the phase shift difference for a cladding RI change $\Delta n_{c}$ of 0.01 . Similarly, changes in the silicon RI have also been investigated by calculating the length required to obtain a phase shift of $\pi$, see Fig. 2e. As in the previous case, it has been obtained at $\lambda_{0}$ wavelength as $L_{\pi}=\pi / \Delta \beta$ where $\Delta \beta$ is the increment in the propagation constants of both modes for a given change in the silicon RI of 0.01. Design 1, marked with a cross in Fig. 2, has been chosen from the hyperplane at the region where both the sensitivity and the $\pi$ length reaches its maximum, and where considerable low values of degradation are obtained. A FoM of 272.2 with a degradation of $1.41 \mathrm{~nm}^{-1}$, a sensitivity of $10,8702 \pi \mathrm{rad}$ and a $\pi$ length of $27.5 \mu \mathrm{m}$ has been obtained for the corrugated waveguide optimization of Design 1.

The second design is presented in Fig. 3a, consisting of a double array of circular holes over a uniform rectangular waveguide, forming a $1 \mathrm{D} \mathrm{PhC}$ in the propagation direction. The dimensions of the unit cell are shown in the right-hand side of Fig. 3a, for the periodicity $a$, waveguide width $w$, distance from the waveguide center to the holes center $d$, hole radius $r$ and height $h$. As in the previous case, the height has been fixed to $220 \mathrm{~nm}$, so that a four-dimensional design space is obtained $(a, w, d, r)$. An initial low-resolution sweep of 256 simulations has been carried out to make a first exploration. In this case, 23 good designs with a FoM above a threshold of 280 are selected for the PCA. Figure $3 \mathrm{~b}$ shows the FoM colormap as a function of $\alpha$ and $\beta$ coefficients. A contour delimiting those good designs with a FoM value higher than 250 is depicted, which demonstrates that this $1 \mathrm{D} \mathrm{PhC}$ design can also be improved in comparison to [35]. It should be also noted that now the maximized area of the FoM is localized on a certain region of the colormap, close to the edge of good designs. Figure 3c shows that these designs present high degradation values due to the deterioration of the band curvature at these regions where FoM is drastically deteriorated. Figure $3 \mathrm{~d}$ and e show the sensitivity and $\pi$ length, 

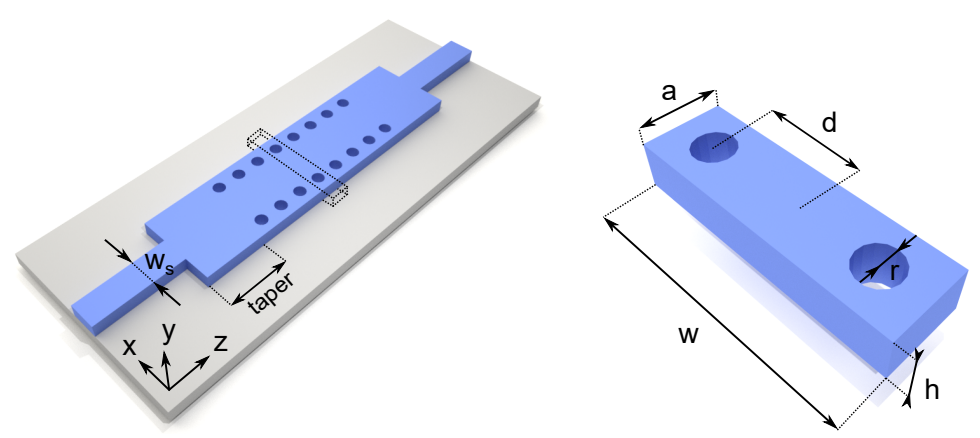
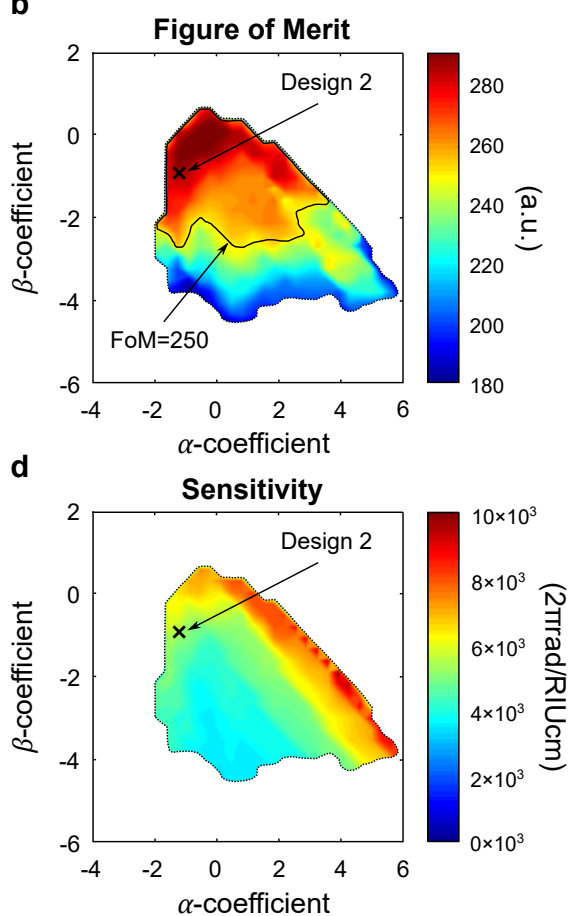
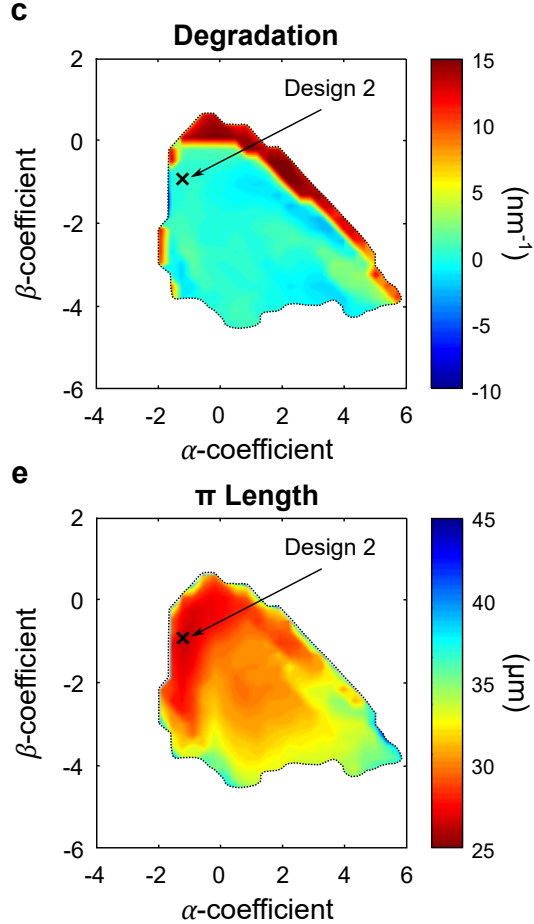

Fig. E.3. Double hole waveguide design optimization. (a) Sketch of the complete slowlight-enhanced interferometer with the unit cell dimensions of the 1D PhC. Colormap of the two principal components of the (b) figure of merit, (c) degradation for deviations of $+-10 \mathrm{~nm}$, (d) normalized sensitivity to $1 \mathrm{~cm}$ and (e) $\pi$ length for a silicon RI increment of 0.01 .

respectively, both calculated at $\lambda_{0}$ wavelength. High-sensitivity designs are obtained near the right-side edge of the hyperplane where high-degradation values were also obtained, while the $\pi$ length is clearly optimized in the opposite left side part of the colormap. Design 2 marked with a cross in Fig. 3 has been chosen from a region of optimized $\pi$ length designs and low degradation. Specifically, a FoM of 284.6 with a degradation of $0.28 \mathrm{~nm}^{-1}$, a sensitivity of $5,3642 \pi \mathrm{rad}$ and a $\pi$ length of $25.9 \mu \mathrm{m}$ has been obtained for the double hole waveguide optimization of Design 2.

Finally, the third 1D PhC design is presented in Fig. 4a and consists of two independent transversal elements periodically placed besides a central uniform 
waveguide, forming a kind of multi-box periodic waveguide in the propagation direction. In this case, a five-dimensional design space is obtained since a small gap is defined within the structure. By using PCA, more complex designs can also be optimized which enables new geometric shapes with new interesting light matter interaction. The unit cell dimensions are shown for the periodicity $a$, central waveguide width $w$, transversal element length $w_{e}$, width $w_{i}$, gap between the central waveguide and the transversal element $g$ and height $h$. The height is fixed to $220 \mathrm{~nm}$ as in previous designs, although in this case a five-dimensional design is obtained. An initial sweep of $3^{5}=243$ simulations is computed to select 42 good designs with a FoM above 260. Figure 4 b shows the FoM

a
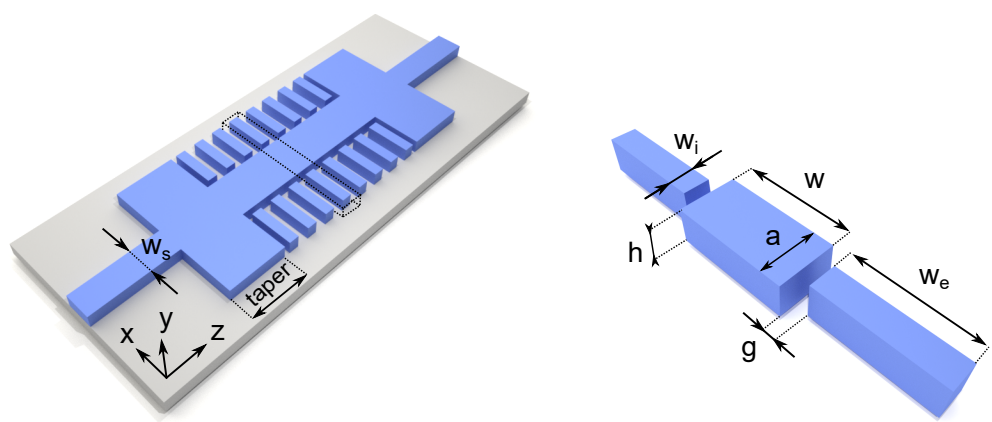

b

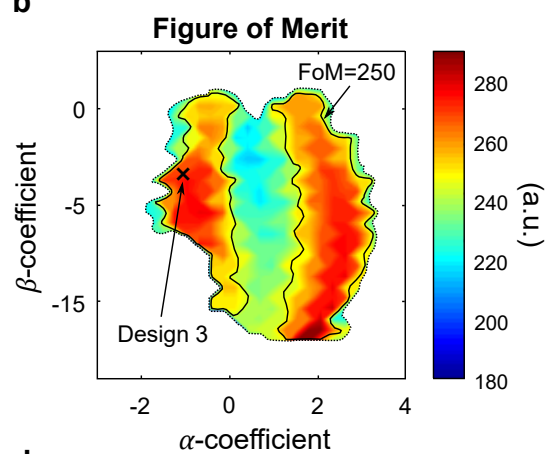

d

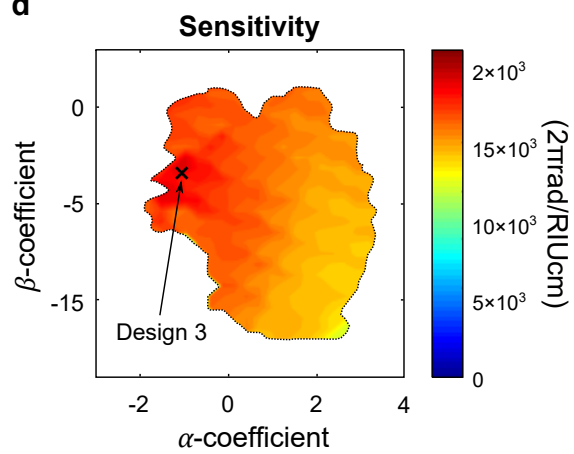

C

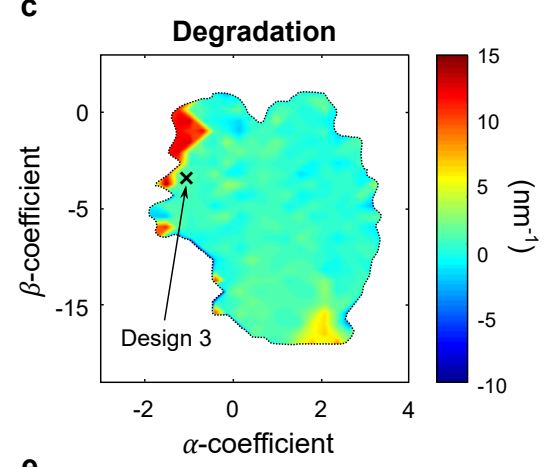

e

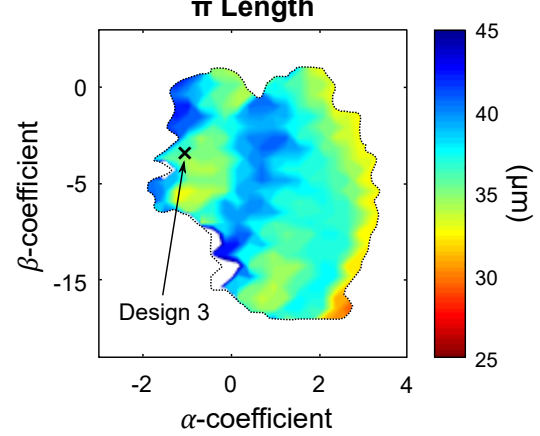

Fig. E.4. Multi box waveguide design optimization. (a) Sketch of the complete slowlight-enhanced interferometer with the unit cell dimensions of the 1D PhC. Colormap of the two principal components of the (b) figure of merit, (c) degradation for deviations of $+-10 \mathrm{~nm}$, (d) normalized sensitivity to $1 \mathrm{~cm}$ and $(\mathrm{e}) \pi$ length for a silicon $\mathrm{RI}$ increment of 0.01 . 
colormap as a function of the first and second principal components where two main regions of designs with a FoM value higher than 250 are obtained. The degradation is equally distributed, see Fig. 4c, while the sensitivity in Fig. 4d is homogeneously distributed and maximized for those designs in the left side part of the FoM colormap. Nevertheless, low-performance regarding the $\pi$ length is obtained for the entire PCA mapping in comparison with the previous design, see Fig. 4e. Therefore, Design 3, marked with a cross in Fig. 4, has been chosen in the region of maximized sensitivity with a FoM of 275.7, degradation of $1.09 \mathrm{~nm}^{-1}$, sensitivity of $19,1962 \pi \mathrm{rad}$ and a $\pi$ length of $36.6 \mu \mathrm{m}$.

\subsubsection{Comparison of the results and discussion}

The resulting optimized designs from the PCA are presented in Table 1, which shows a comparison between the main results regarding the FoM, fabrication deviations and changes in the RI of the cladding and the core of the structure. Dimensions are sorted in the appearance order in which they have been previously detailed. Note that all designs present a higher FoM value than the one reported in Ref. [35], which demonstrates an enhancement of the optical path in slow-light-enhanced bimodal waveguides by using PCA. Moreover, further improvement in terms of both sensitivity and $\pi$ length is presented. Compared to [35], a specific $22 \%$ reduction in the physical length of the interferometer as a modulator (changes in the silicon RI) is obtained for Design 2, whereas the sensitivity is increased by a factor of 1.85 for Design 3, acting as a sensor (changes in the cladding RI). These results are obtained for an all-dielectric structure compared to other highly sensitive plasmonic sensors based on metallic structures [6-9]. However, some other parameters that could affect the sensitivity (other material properties, temperature changes or fabrication deviations) must be considered in future sensor optimization steps. By reducing the design space dimensionality, we develop a parallel optimization for both the optical path and the light matter interaction of the interferometer. Furthermore, this method has been used in three different geometrical designs to further evaluate the field distribution and its interaction with induced RI changes. As a result, very different performance are obtained for the configurations considered, as in the case of the silicon and cladding RI sensitivity contrast between Designs 2 and 3.

Figure 5a shows the first three bands for the TE-like polarization for the three designs presented in Table 1. The region of interest is located around $1550 \mathrm{~nm}$ at the third band curvature, marked with the green shaded area of Fig. 5a. As it was schematically explained in Fig. 1, in this region an anti-crossing point is formed, below which the bimodal desired operation is obtained, see $\beta_{1,2}$ in Fig. $5 \mathrm{a}$. Note that the operating region could be roughly 
Table 4.1 PCA Optimized designs comparison

\begin{tabular}{ccccccc}
\hline Design & $\alpha, \beta$ & Dimensions $(\mathrm{nm})$ & FoM & Deg. $\left(\mathrm{nm}^{-1}\right)$ & $\mathrm{S}(2 \pi \mathrm{rad})$ & $\mathrm{L}_{\pi}(\mu \mathrm{m})$ \\
\hline Ref. [35] & None & {$[370,600,1400,220]$} & 249.8 & 0.62 & 10,337 & 33 \\
1 (corr.) & {$[-0.93,-1.31]$} & {$[378,560,1638,171]$} & 272.2 & 1.41 & 10,870 & 27.5 \\
2 (hol.) & {$[-1.24,-1.03]$} & {$[387,1319,439,100]$} & 284.6 & 0.28 & 5,364 & 25.6 \\
3 (box.) & {$[-0.96,-3.17]$} & {$[360,726,722,168,90]$} & 275.7 & 1.09 & 19,196 & 36.6 \\
\hline
\end{tabular}

a

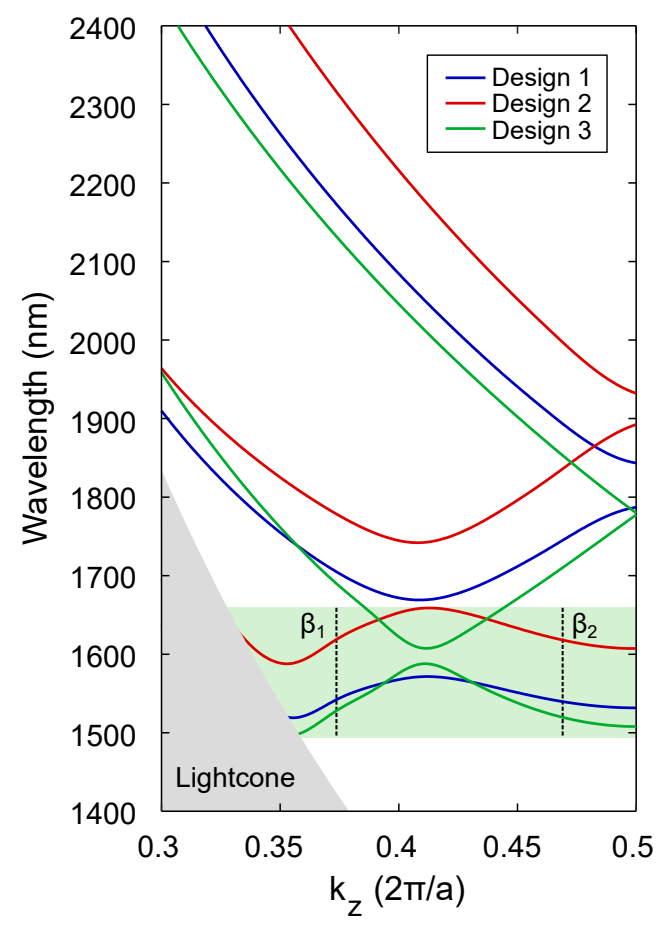

b

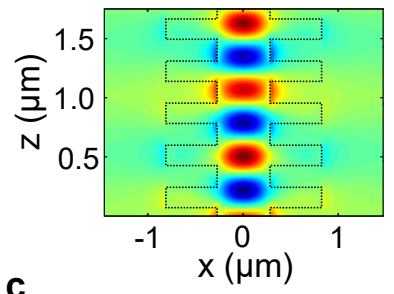

c
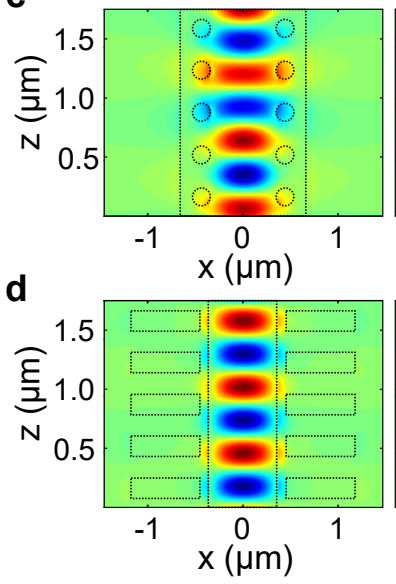

$\beta_{2}$
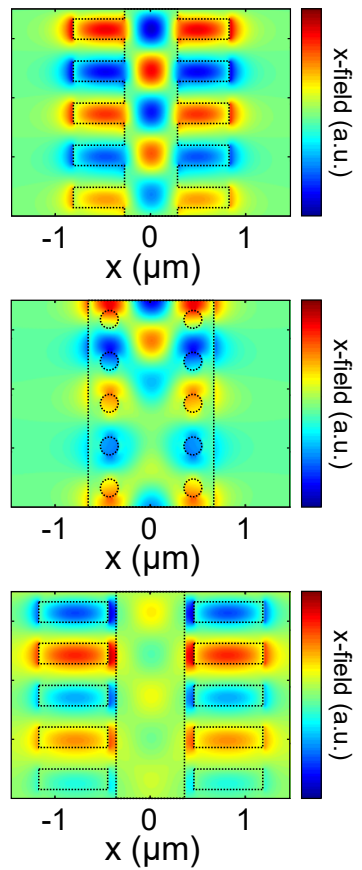

Fig. E.5. (a) First three TE-like polarized bands of the band diagram for the optimized designs in the PCA. The green shaded area represents the bimodal region. (b) Real part of the $x$-component field in the corrugated waveguide design for the fundamental mode at $k_{z}=0.3752 \pi / a$ and the higher order mode at $k_{z}=0.4752 \pi / a$, left and right colormaps, respectively. (c) Field distribution in the double hole waveguide and (d) multibox waveguide designs. The dashed line represents the silicon structure shape.

designed to other wavelengths by simply changing the periodicity of the structure and re-scaling the rest of parameters. The band structure formation for all designs is very similar, specifically the curvature of the third band, in accordance with the FoM results obtained in Table 1. Figure 5b, $\mathrm{c}$ and d show the field distribution for Designs 1, 2 and 3, respectively. Both the fundamental $\left(\beta_{1}\right)$ and the higher order $\left(\beta_{2}\right)$ modes are shown. In all these cases, the fundamental mode is highly confined within the center of the waveguide, while the higher order mode is partially localized in the periodic pattern of the structure. Note that in the right graph of Fig. $5 \mathrm{c}$, the $\beta_{2}$ mode is more confined within the silicon 
structure than in the case of the right graph of Fig. 5d, where a high part of the field is localized within the gap. This fact explains why Design 2 is highly sensitive for changes in the silicon RI and Design 3 for changes in the cladding RI, and thus their difference between the sensitivity and $\pi$ length results.

For the sake of completeness, the results have also been validated with 3D finite difference time domain (FDTD) simulations using the CST Studio software. In this case, the unit cells are periodically disposed in the propagation direction with its correspondent taper configuration of $\sim 1.5 \mu \mathrm{m}$ length and accessed in and out with a single mode waveguide of $450 \mathrm{~nm}$ width. The fundamental TE mode of the input waveguide excites both modes in the bimodal $\mathrm{PhC}$, which similarly contribute to the excitation of the fundamental TE mode at the output waveguide. Thus, the bimodal interference pattern can be observed in the transmitted spectra. Note that as in the case of [35], there will be a trade-off between the group index and the bimodal bandwidth. To measure the phase shift, we need to calculate the relationship between the wavelength shift of a constructive interference in the spectrum and the free spectral range (FSR) by using the following expression: $\Delta \phi=\Delta \lambda / F S R$. Figure $6 \mathrm{a}$ and $\mathrm{b}$ depict the phase shift of the lowest wavelength interference for all designs as a function of linear changes in the cladding and silicon RI, respectively.

Note that the most sensitive configuration to cladding changes in Design 3, which is in a good agreement with the results shown in Table 1. Likewise, Design 2 is the most sensitive to silicon RI variations, hence the lower $\pi$ length is obtained for this configuration, although it presents a lower extinction ratio $(2.5 \mathrm{~dB})$ than the other configurations (15-20 dB) because of the low coupling efficiency to the slow light mode that must be addressed in a future optimization of the taper design. Design 1 presents a balanced behavior for changes in both the cladding and core, which also matches with previous results. Figure $6 \mathrm{~b}$ shows the considered interference in the transmitted spectra for all designs under cladding RI changes. The FSR is almost equal in all of them, while the wavelength shift is clearly higher for Design 3 (green right-side graph), which explains the results of Fig. 6a. Similarly, Fig. 6d shows the spectra for a change in the silicon core RI of 0.01 and for 1D PhCs with the $\pi$ lengths calculated in the PCA. The FDTD results perfectly match previous MPB simulations, an a clear $\pi$ phase shift is observed in the spectra.

\subsubsection{Conclusions}

We have demonstrated a method to design and optimize slow-light-enhanced bimodal interferometers by using dimensionality reduction techniques. A FoM has been introduced to characterize the bimodal band curvature of the 1D PhC and PCA has been employed to optimize the optical path of the interferometers in terms of group index 
and bandwidth. A low-dimensional 2D hyperplane containing the optimized designs has been obtained, which allows us to explore different performance criteria as the degradation to fabrication deviations, bulk sensitivity and $\pi$ length for changes in the silicon RI. As a result, three different single-channel 1D PhC interferometers have been designed, with remarkable improvements regarding other similar slow-light-enhanced bimodal structures [35]. Specifically, simulations show that the physical interferometer

a

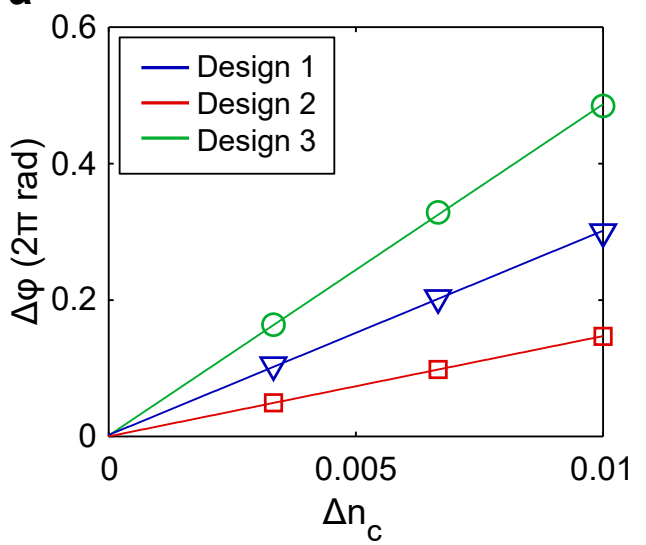

C

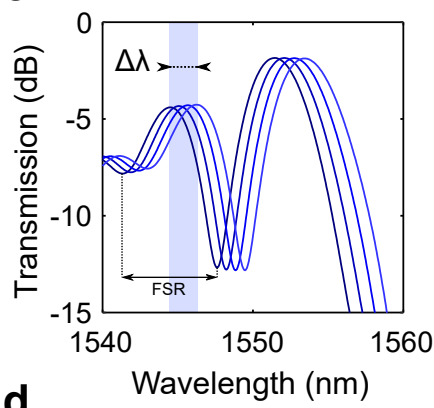

d

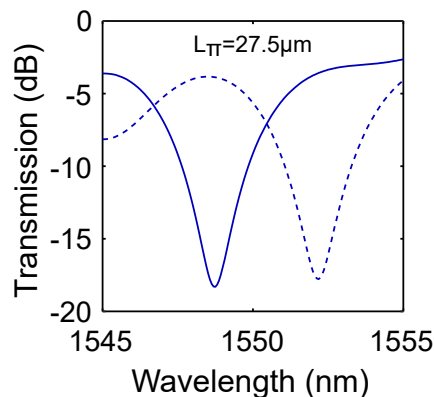

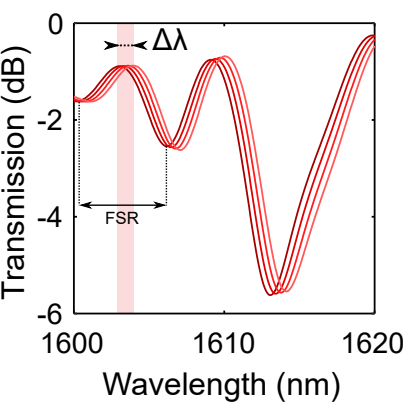

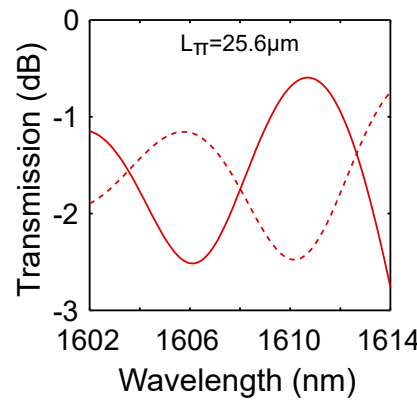

b

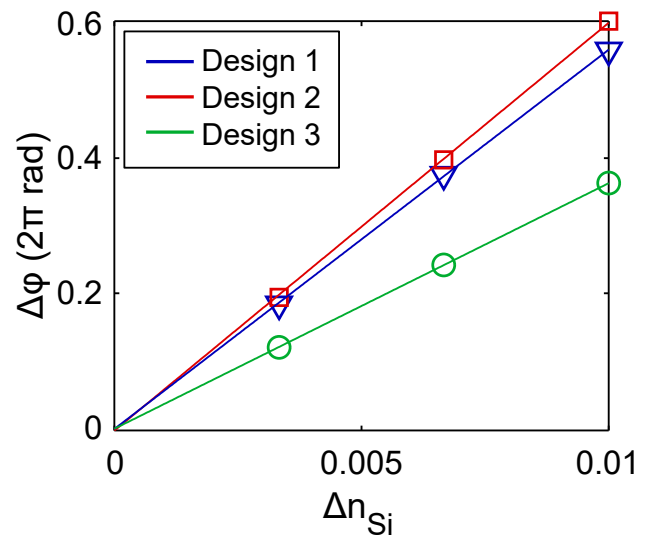

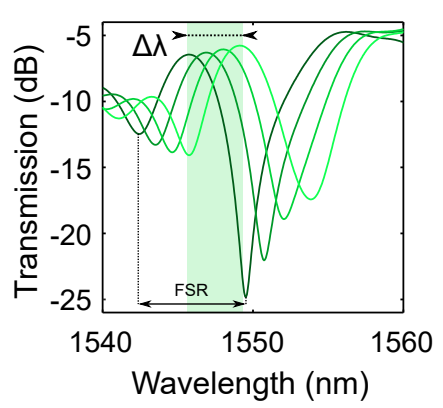

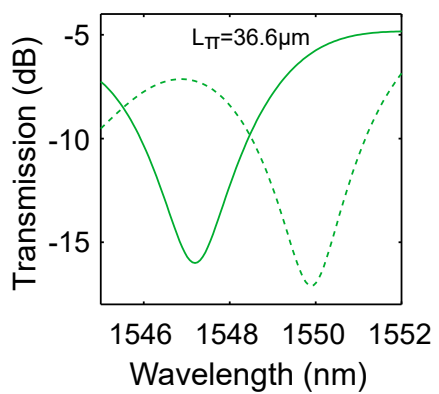

Fig. E.6. Comparison of the optimized designs in FDTD simulations. Phase shift of the first constructive interference for (a) changes in cladding and (b) in the silicon refractive index. (c) Transmission spectra of the slow light bimodal interferometers with $\mathrm{N}=100$ periods and for different cladding RI (a linear sweep from 1.444 to 1.454). All PCA optimized configurations are shown, Design 1 (blue colored left graph), Design 2 (red colored middle graph of Fig. 6c), and Design 3 (green colored right graph). (d) Transmission spectra for all PCA optimized designs with a length $L_{\pi}$. Solid lines represents the spectra for a RI index of 3.4777 and dashed line for 3.4877 . 
modulation length has been reduced by $22 \%$ for silicon RI variations, which means that this device can be integrated in an all-dielectric structure of only $33 \mu \mathrm{m}^{2}$ footprint. In comparison with the literature, these results correspond to a reduction of more than two orders of magnitude respect to conventional MZI structures [12] and more than one order compared to slow-light based interferometers [28, 31]. Moreover, a sensor with values of $19.2 \times 10^{3} 2 \pi \mathrm{rad} / \mathrm{RIU} \cdot \mathrm{cm}$ has been reported, which means nearly twice as high as cited slow-light-enhanced bimodal interferometers [35], and more than one order of magnitude compared to standard MZI sensors and bimodal waveguides [5, 24]. Overall, these findings open up new avenues to design and optimize bimodal photonic crystal devices which may lead to novel applications in different areas with enhanced properties.

\section{Fundings}

The authors acknowledge funding from the Generalitat Valenciana through the AVANTI/ 2019/123, ACIF/2019/009 and PPC/2020/037 grants, from the Spanish Government through the PID2019-106965RB-C21-PHOLOW project and from the European Union through the operational program of the European Regional Development Fund (FEDER) of the Valencia Regional Government 2014-2020.

\section{Disclosures}

The authors declare no conflicts of interest.

\section{Data availability.}

Data underlying the results presented in this paper are not publicly available at this time but may be obtained from the authors upon reasonable request.

\subsubsection{References}

1. G. T. Reed, G. Mashanovich, F. Y. Gardes, and D. J. Thomson, "Silicon optical modulators," Nat. Photonics 4, 518-526 (2010).

2. S. Kumar, S. K. Raghuwanshi, and A. Kumar, "Implementation of optical switches using Mach-Zehnder interferometer,” Opt. Eng. 52, 097106- 097119 (2013).

3. P. Kozma, F. Kehl, E. Ehrentreich-Förster, C. Stamm, and F. F. Bier, "Integrated planar optical waveguide interferometer biosensors: A comparative review," Biosens. Bioelectron. 58, 287-307 (2014). 
4. W. Bogaerts, D. Pérez, J. Capmany, D. A. Miller, J. Poon, D. Englund, F. Morichetti, and A. Melloni, "Programmable photonic circuits," Nature 586, 207-216 (2020).

5. A. Calle, A. Llobera, C. Dom, F. Prieto, B. Sepúlveda, A. Calle, A. Llobera, C. Domínguez, A. Abad, A. Montoya, and L. Lechuga, "An integrated optical interferometric nanodevice based on silicon technology for biosensor applications A Abad , A Montoya and L M Lechuga," Online 14, 907-912 (2003).

6. S. I. Bozhevolnyi, V. S. Volkov, E. Devaux, J. Y. Laluet, and T. W. Ebbesen, "Channel plasmon subwavelength waveguide components including interferometers and ring resonators," Nature 440, 508-511 (2006).

7. Y. Gao, Q. Gan, Z. Xin, X. Cheng, and F. J. Bartoli, "Plasmonic Mach-Zehnder interferometer for ultrasensitive on-chip biosensing,” ACS Nano 5, 9836-9844 (2011).

8. . H. T. Chorsi, Y. Lee, A. Alù, and J. X. Zhang, "Tunable plasmonic substrates with ultrahigh Q-factor resonances,” Sci. Reports 7, 1-9 (2017).

9. H. T. Chorsi, Y. Zhu, and J. X. Zhang, "Patterned Plasmonic Surfaces-Theory, Fabrication, and Applications in Biosensing," J Microelectromech Syst 176, 139-148 (2017).

10. P. Muñoz, G. Micó, L. A. Bru, D. Pastor, D. Pérez, J. D. Doménech, J. Fernández, R. Baños, B. Gargallo, R. Alemany, A. M. Sánchez, J. M. Cirera, R. Mas, and C. Domínguez, "Silicon nitride photonic integration platforms for visible, near-infrared and mid-infrared applications," Sensors (Switzerland) 17, 1-25 (2017).

11. A. Liu, R. Jones, L. Liao, D. Samara-Rubio, D. Rubin, O. Cohen, R. Nicolaescu, and M. Paniccia, "A high-speed silicon optical modulator based on a metal-oxide-semiconductor capacitor," Nature 427, 615-618 (2004).

12. L. Liao, D. Samara-Rubio, M. Morse, A. Liu, D. Hodge, D. Rubin, U. D. Keil, and T. Franck, "High speed silicon Mach-Zehnder modulator," Opt. Express 13, 3129-3135 (2005).

13. W. M. Green, M. J. Rooks, L. Sekaric, and Y. A. Vlasov, "Ultra-compact, low RF power, 10 Gb/s silicon Mach-Zehnder modulator,” Opt. Express 15, 17106-17113 (2007).

14. M. W. Geis, S. J. Spector, R. C. Williamson, and T. M. Lyszczarz, "Submicrosecond submilliwatt silicon-on-insulator thermooptic switch," IEEE Photonics Technol. Lett. $16,2514-2516$ (2004).

15. P. Sun and R. M. Reano, "Submilliwatt thermo-optic switches using free-standing silicon-on-insulator strip waveguides,” Opt. Express 18, 8406-8411 (2010). 
16. M. R. Watts, J. Sun, C. Derose, D. C. Trotter, R. W. Young, and G. N. Nielson, "Adiabatic thermo-optic Mach-Zehnder switch," Opt. Lett. 38, 733-735 (2013).

17. Q. Liu, X. Tu, K. W. Kim, J. S. Kee, Y. Shin, K. Han, Y. J. Yoon, G. Q. Lo, and M. K. Park, "Highly sensitive Mach-Zehnder interferometer biosensor based on silicon nitride slot waveguide," Sensors Actuators, B: Chem. 188, 681-688 (2013).

18. X. Sun, D. Dai, L. Thylén, and L.Wosinski, "High-sensitivity liquid refractive-index sensor based on a Mach-Zehnder interferometer with a double-slot hybrid plasmonic waveguide," Opt. Express 23, 25688-25699 (2015).

19. P. J. Bock, P. Cheben, J. H. Schmid, J. Lapointe, A. Delâge, S. Janz, G. C. Aers, D.-X. Xu, A. Densmore, and T. J. Hall, "Subwavelength grating periodic structures in silicon-on-insulator: a new type of microphotonic waveguide," Opt. Express 18, 20251-20262 (2010).

20. R. Sumi, N. Das Gupta, and B. K. Das, "Integrated optical Mach-Zehnder interferometer with a sensing arm of sub-wavelength grating waveguide in SOI," Proc. IEEE Sensors pp. 1-3 (2017).

21. D. M. Kita, J. Michon, S. G. Johnson, and J. Hu, "Are slot and sub-wavelength grating waveguides better than strip waveguides for sensing?" Optica 5, 1046-1054 (2018).

22. V. J. Sorger, N. D. Lanzillotti-Kimura, R. M. Ma, and X. Zhang, "Ultra-compact silicon nanophotonic modulator with broadband response,” Nanophotonics 1, 17-22 (2012).

23. A. Densmore, S. Janz, R. Ma, J. H. Schmid, D.-X. Xu, A. Delâge, J. Lapointe, M. Vachon, and P. Cheben, "Compact and low power thermo-optic switch using folded silicon waveguides," Opt. Express 17, 10457-10465 (2009).

24. K. E. Zinoviev, A. B. González-Guerrero, C. Domínguez, and L. M. Lechuga, "Integrated bimodal waveguide interferometric biosensor for label-free analysis,” J. Light. Technol. 29, 1926-1930 (2011).

25. L. Torrijos-Morán and J. García-Rupérez, "Single-channel bimodal interferometric sensor using subwavelength structures,” Opt. Express 27, 8168-8179 (2019).

26. L. Torrijos-Morán, A. Griol, and J. García-Rupérez, "Experimental study of subwavelength grating bimodal waveguides as ultrasensitive interferometric sensors," Opt. Lett. 44, 4702-4705 (2019).

27. N. Shaw,W. Stewart, J. Heaton, and D.Wight, "Optical slow-wave resonant modulation in electro-optic GaAs/AlGaAs modulators,” Electron. letters 35, 1557-1558 (1999). 
28. M. Soljacic, S. G. Johnson, S. Fan, M. Ibanescu, E. Ippen, and J. D. Joannopoulos, "Photonic-crystal slow-light enhancement of nonlinear phase sensitivity," J. Opt. Soc. Am. B 19, 2052-2059 (2002).

29. Y. Jiang, W. Jiang, L. Gu, X. Chen, and R. T. Chen, "80-Micron Interaction Length Silicon Photonic Crystal Waveguide Modulator,” Appl. Phys. Lett. 87, 1-3 (2005).

30. L. O’Faolain, D. M. Beggs, T. P. White, T. Kampfrath, K. Kuipers, and T. F. Krauss, "Compact optical switches and modulators based on dispersion engineered photonic crystals," IEEE Photonics J. 2, 404-414 (2010).

31. A. Brimont, D. J. Thomson, P. Sanchis, J. Herrera, F. Gardes, J. M. Fedeli, G. T. Reed, and J. Martí, "High speed silicon electro-optical modulators enhanced via slow light propagation,” Opt. Express 19, 20876-20885 (2011).

32. K. Qin, S. Hu, S. T. Retterer, I. I. Kravchenko, and S. M. Weiss, "Slow light Mach-Zehnder interferometer as label-free biosensor with scalable sensitivity,” Opt. Lett. 41, 753-756 (2016).

33. E. A. Camargo, H. M. H. Chong, and R. M. De La Rue, "2D Photonic crystal thermo-optic switch based on AlGaAs/GaAs epitaxial structure,” Opt. Express 12, 588-592 (2004).

34. H. Nakamura, Y. Sugimoto, K. Kanamoto, and N. Ikeda, "Ultra-fast photonic crystal/quantum dot all-optical switch for future photonic networks," Opt. Express 12, 6606-6614 (2004).

35. L. Torrijos-Morán, A. Griol, and J. García-Rupérez, "Slow light bimodal interferometry in one-dimensional photonic crystal waveguides," Light. Sci. \& Appl. 10, 16 (2021).

36. L. Torrijos-Morán, A. Brimont, A. Griol, P. Sanchis, and J. García-Rupérez, "Ultra-compact optical switches using slow light bimodal silicon waveguides," J. Light. Technol. 39, 3495-3501 (2021).

37. A. Håkansson and J. Sánchez-Dehesa, "Inverse designed photonic crystal de-multiplex waveguide coupler," Opt. Express 13, 5440-5449 (2005).

38. J. Covey and R. T. Chen, "Efficient perfectly vertical fiber-to-chip grating coupler for silicon horizontal multiple slot waveguides,” Opt. Express 21, 10886-10896 (2013).

39. J. S. Jensen and O. Sigmund, "Systematic design of photonic crystal structures using topology optimization: Low-loss waveguide bends," Appl. Phys. Lett. 84, 2022-2024 (2004). 
40. A. C. Niederberger, D. A. Fattal, N. R. Gauger, S. Fan, and R. G. Beausoleil, "Sensitivity analysis and optimization of sub-wavelength optical gratings using adjoints," HP Lab. Tech. Rep. 22, 1207-1216 (2014).

41. Y. Ma, Y. Zhang, S. Yang, A. Novack, R. Ding, A. E.-J. Lim, G.-Q. Lo, T. Baehr-Jones, and M. Hochberg, "Ultralow loss single layer submicron silicon waveguide crossing for SOI optical interconnect,” Opt. Express 21, 29374-29382 (2013).

42. T. Watanabe, M. Ayata, U. Koch, Y. Fedoryshyn, and J. Leuthold, "Perpendicular Grating Coupler Based on a Blazed Antiback-Reflection Structure,” J. Light. Technol. 35, 4663-4669 (2017).

43. J. Peurifoy, Y. Shen, L. Jing, Y. Yang, F. Cano-Renteria, B. Delacy, M. Tegmark, J. D. Joannopoulos, and M. Soljacic, "Nanophotonic particle simulation and inverse design using artificial neural networks," Aci Adv pp. 1-8 (2017).

44. M. Turduev, E. Bor, C. Latifoglu, I. H. Giden, Y. Sinan Hanay, and H. Kurt, "Ultracompact Photonic Structure Design for Strong Light Confinement and Coupling Into Nanowaveguide," J. Light. Technol. 36, 2812-2819 (2018).

45. M. Liang, Y. Li, H. Meng, M. A. Neifeld, and H. Xin, "Reconfigurable array design to realize principal component analysis (PCA)-based microwave compressive sensing imaging system,” IEEE Antennas Wirel. Propag. Lett. 14, 1039-1042 (2015).

46. D. Melati, Y. Grinberg, M. Kamandar Dezfouli, S. Janz, P. Cheben, J. H. Schmid, A. Sánchez-Postigo, and D. X. Xu, "Mapping the global design space of nanophotonic components using machine learning pattern recognition," Nat. Commun. 10, 1-9 (2019).

47. D. Melati, M. Dezfouli, Y. Grinberg, J. H. Schmid, R. Cheriton, S. Janz, P. Cheben, and D. $\mathrm{X} . \mathrm{Xu}$, "Design of Compact and Efficient Silicon Photonic Micro Antennas with Perfectly Vertical Emission,” IEEE J. Sel. Top. Quantum Electron. 27, 1-10 (2021).

48. A. Waqas, P. Manfredi, and D. Melati, "Performance Variability Analysis of PhotonicCircuits with Many Correlated Parameters," J. Light. Technol. 8724, 1-8 (2021).

49. M. Notomi, K. Yamada, A. Shinya, J. Takahashi, C. Takahashi, and I. Yokohama, "Extremely large group-velocity dispersion of line-defect waveguides in photonic crystal slabs,” Phys. Rev. Lett. 87, 253902-253904 (2001).

50. S. Johnson and J. Joannopoulos, "Block-iterative frequency-domain methods for Maxwell's equations in a planewave basis,” Opt. Express 8, 173-190 (2001). 
51. J. P. Hugonin, P. Lalanne, T. P. White, and T. F. Krauss, "Coupling into slow-mode photonic crystal waveguides," Opt. Lett. 32, 2638-2640 (2007). 



\section{Chapter 5}

\section{Discussion and conclusions}

Once the original contributions have been presented, in this last chapter we highlight the main results reported throughout the thesis, as well as discuss the principal findings of the work and relate them with the initial objectives stated at the introduction. We also detail next steps that will be developed in future works and conclude the document with some final remarks.

\subsection{General discussion of the results}

In this section we present a brief discussion on the main results reported. We will consider separately contributions on subwavelength structures and photonic crystals as they provide different solutions to the main goal of optical interferometry in a single-channel waveguides. We will also compare our designs with the state-of-the-art of similar devices, in order to illustrate the main advantages in the resulting footprint and operational features.

\subsubsection{Bimodal subwavelength waveguides}

The first part of the thesis is about bimodal SWG waveguides for spectral-based refractive index sensing. As it is described in the introduction of Paper A and Paper B, SWG are attracting increasing interest from the scientific community in the last years due to their dispersion and anisotropy properties that provide broadband operation and compactness. Figure 5.1 shows some photonic examples of devices made of multimode SWGs $[154,101]$. Specifically, a directional coupler and a MMI for beam splitting are depicted, as well as our proposed bimodal refractive index sensor. In our case, we benefit from the dispersion of the fundamental mode to critically enhance the spectral sensitivity. 

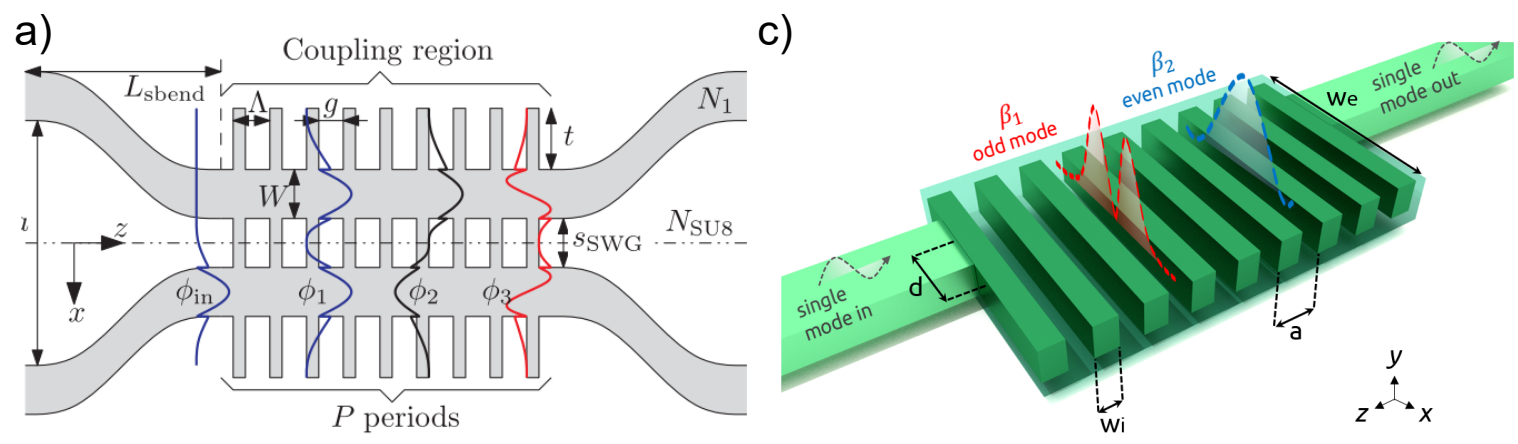

b)

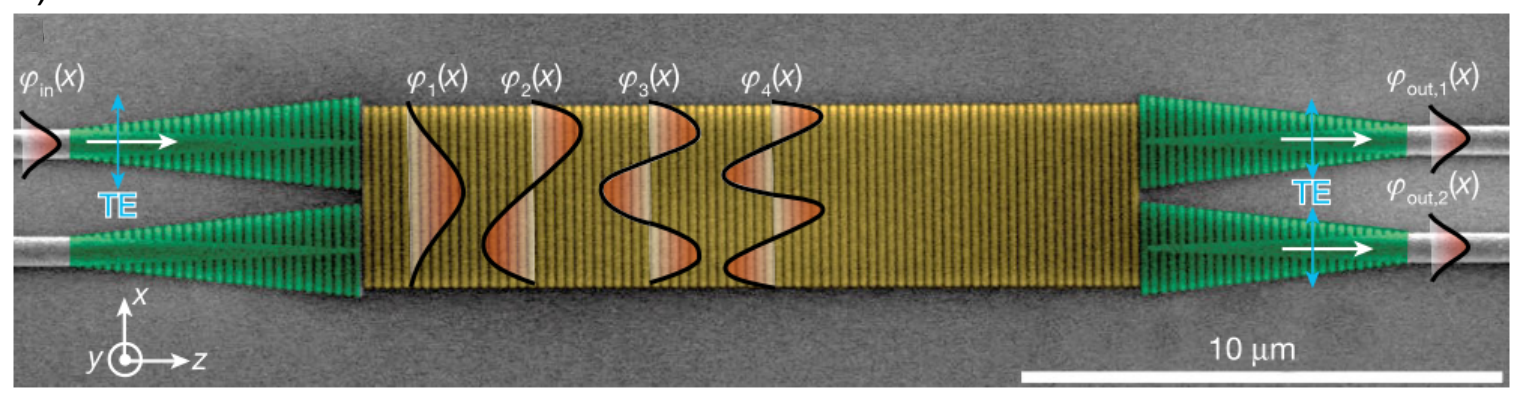

Fig. 5.1. SWG designs based on multimode operation for different photonic applications such as (a) a directional coupler [154] (b) beam splitter [101] and (c) our proposed bimodal refractive index sensor.

This fact, combined with the advantages of SWGs for sensing, provides outstanding results in terms of bulk and surface sensitivity. Figure 5.2 shows some other photonic sensing structures on SWG based waveguides [155, 156, 115]. In these cases, single-mode operation is employed but it allows us to illustrate other approaches involving SWGs to increase the light-matter interaction with the cladding, and thus the sensitivity. Our SWG elements are similar to the examples herein presented, but in our case to design a bimodal sensor for spectral-based sensing.

The presented sensor configuration clearly presents dispersion for the even mode at the region near the end of the Brillouin zone, specifically at lower wavelengths, where the structure might be considered in between the PhC and the SWG regimes. On the other hand, for higher wavelengths the structure is in the deep subwavelength regime with less dispersive modes, especially in the case of the odd mode, although the even one is still slightly dispersive. This transition between dispersive and non-dispersive regions provokes a soft non-linear fall in the phase shift between both modes, which creates the so-called critical sensitivity region. Moreover, these dispersion effects can also be observed in the transmission measurements where the spectral dips are more grouped for low bimodal wavelengths in which the structure operates near the $\mathrm{PhC}$ regime. Another interesting property is the scalability of SWG structures to operate in other wavelength ranges. The operating region of the bimodal structure can be moved to other regions by 
correctly designing the period $\Lambda$ of the structure. For instance, by reducing the period of the SWG structure we obtain new bands shifted to lower wavelengths for even and odd modes, thus moving the operating wavelength. However, we should take into account that for lower wavelengths we approximate to the light cone, where the modes become lossy and the bimodal condition cannot be reached.

The presence of fabrication deviations could affect in different ways depending on which design parameter could suffer from this variability. If the parameter $\Lambda$ is affected, the spectral response will be moved to other operating wavelengths, but this would not be so crucial since the spectra response will be shifted just a few nanometers. Another parameter that could be affected is the width of the SWG element $w$. Given that there is a good agreement between simulations and experimental measurements, the variation of the width do not affect that much in the modal behavior of the structure since we obtain similar results for simulated widths from $1300 \mathrm{~nm}$ to $1500 \mathrm{~nm}$. Nonetheless, the most important parameter that has to be carefully controlled in fabrication processes is the thickness of the SWG element, which determines the duty cycle of the structure. As it has been reported in the manuscript, the sensitivity is strongly dependent on the duty cycle, so that variations in this parameter could affect the performance of the sensor.

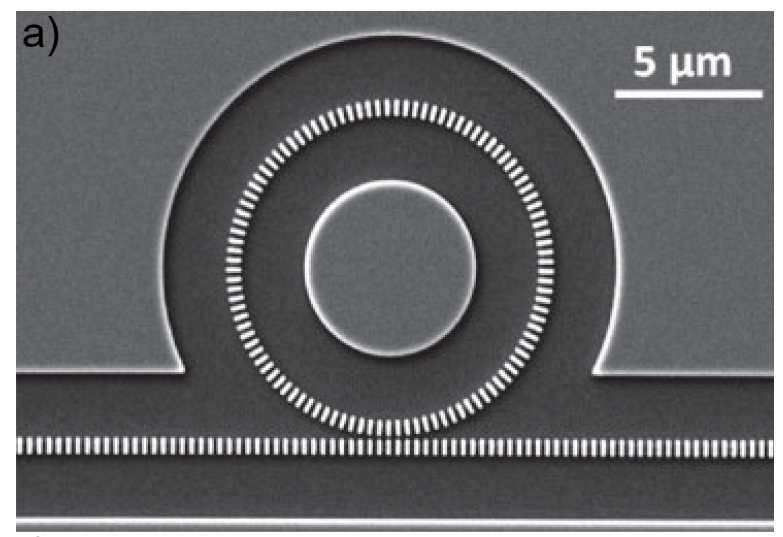

c)
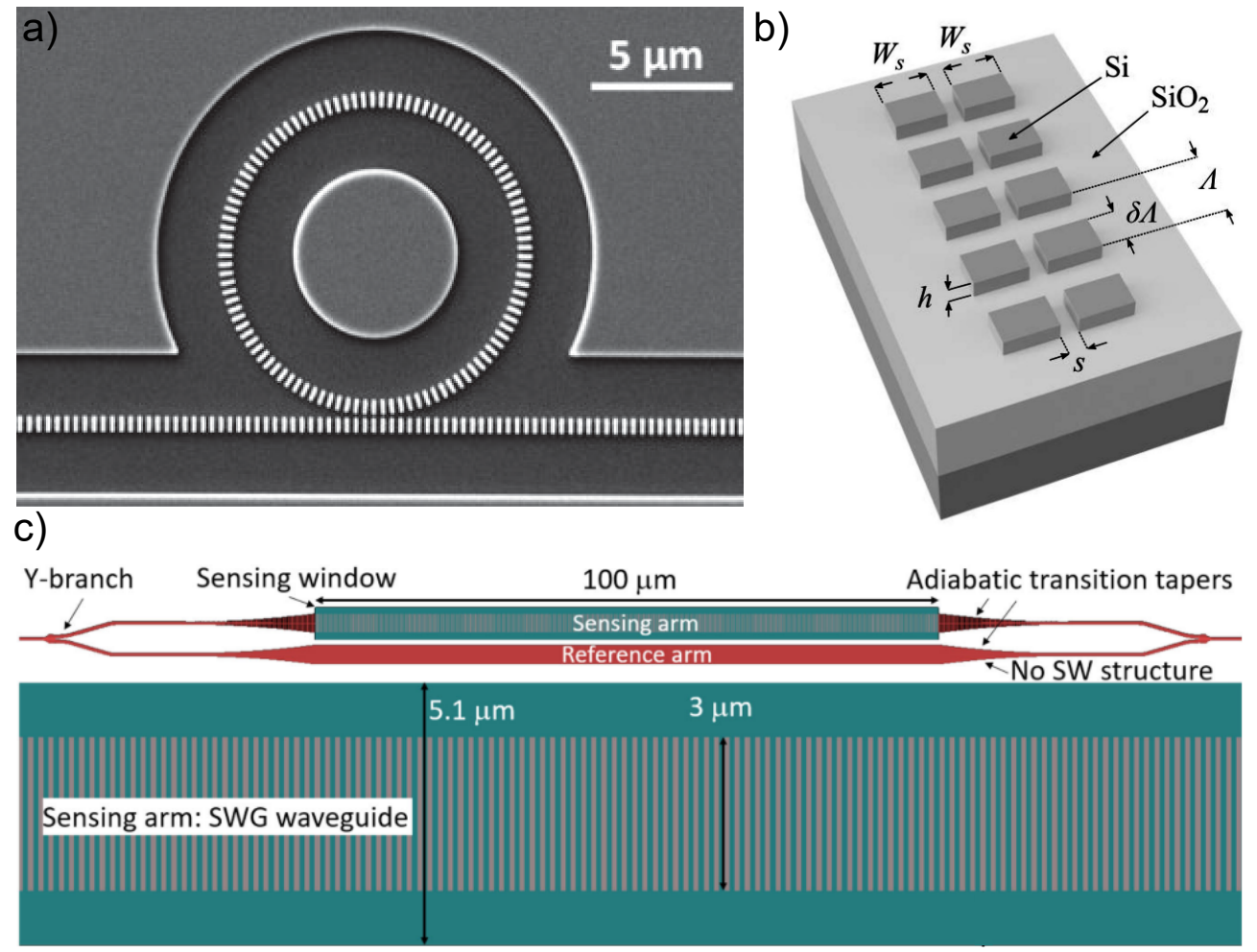

Adiabatic transition tapers

No SW structure

Fig. 5.2. SWG based refractive index sensors consisting of (a) ring resonators [155] (b) slot waveguides [156] and (c) MZI configurations [115]. 
It is worth also noting that the sensor length strongly influences the number of spectral dips in the critical sensitivity region, but not the sensitivity of those peaks. In addition, having different $N$ periods for two different configurations allows us to demonstrate this fact, which is not exclusive of this type of SWG interferometer. The non-dependence of the sensitivity with the sensor length is a common feature to all MZI schemes (including bimodal sensors) interrogated with a wavelength sweep. Regarding the losses for different SWG lengths, the propagation losses are typically higher than in a normal solid-core waveguides, around $10-25 \mathrm{~dB} / \mathrm{cm}$. However, one of the objectives of our sensor proposal is reducing the total length of the sensing structure in comparison with other interferometric configurations. In that way, we can work with sub-mm structures (instead of requiring several millimeters or even centimeters lengths), what would introduce losses below 1 or $2 \mathrm{~dB}$. The limit of detection has not been specifically studied because it depends on further optimization of the presented designs that must be addressed in future works. However, a rough estimation of the actual limit of detection value is around $10^{-5}$ according to the values provided in [Conf. 4]. In fact, increasing the sensor length would produce a higher number of peaks with a sharper spectral response and a higher quality factor that would be easier to track. That reduction of the noise/uncertainty is translated into a reduction of the limit of detection which would be an advantage, but not to an improvement of the spectral sensitivity, which remains the same regardless the sensor length.

\subsubsection{Bimodal 1D photonic crystals}

The second part of the thesis includes bimodal photonic crystals as common path interferometers with reduced footprints (Paper C, Paper D and Paper E). To illustrate the contribution of these findings to the state-of-the-art we must take a look at the interferometric configurations commonly used as modulators and switches [121, 130, 30, 134]. Figure 5.3 shows some interferometric schemes aiming at improving the performance by employing extremely long paths in folded waveguides, or including photonic crystal structures in the MZI arms. In all cases, the main objective is the same, engineering very long optical paths to increase the measured phase shift at the output. On this matter, our bimodal interferometers provide high-performance slow light operation while addressing size limitations typical of the aforementioned interferometric architectures. Moreover, these findings can be extrapolated to sensing devices, where bimodal waveguides and MZI including slow light structures have been widely demonstrated as biosensors, see Fig. 5.4a and b, respectively [32, 133]. Our approach combines these two ideas into a straightforward design that can be easily integrated in mass-volume PICs. 
Regarding the losses, light coupling in a PhC waveguide becomes inefficient when dealing with both slow light and higher order modes. In both Paper $\mathrm{C}$ and Paper $\mathrm{D}$ a 1D PhC waveguide supporting the fundamental mode and the higher order odd mode is presented. On the one hand, there is a group index mismatch between the fundamental mode of the input/output waveguide and the slow light mode supported by the PhC structure, which causes a Fresnel reflection at the interface. This is translated into a lower coupling efficiency of the slow light mode, and subsequently, into higher insertion losses. This fact explains the increasing losses presented in the experimental measurements of the bimodal PhCs where both insertion loss and extinction ratio deteriorate at the slow light region. However, there is another fact that contributes to the increment of the insertion loss. That is the field mismatch between the fundamental mode of the input/output waveguide and the higher order mode of the PhC structure. The higher order odd mode presents a different field distribution within the $\mathrm{PhC}$ which leads to a lower coupling, and hence an increment of the insertion losses measured at the region of high group index contrast. The taper placed at the interface between the input/output waveguides and the $\mathrm{PhC}$ waveguide is intended to improve the coupling efficiency of the higher order mode. Nevertheless, further work improving the bimodal excitation

a)

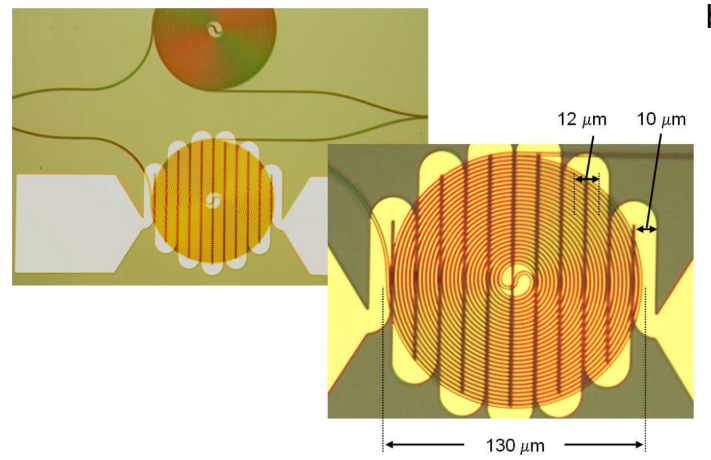

c)

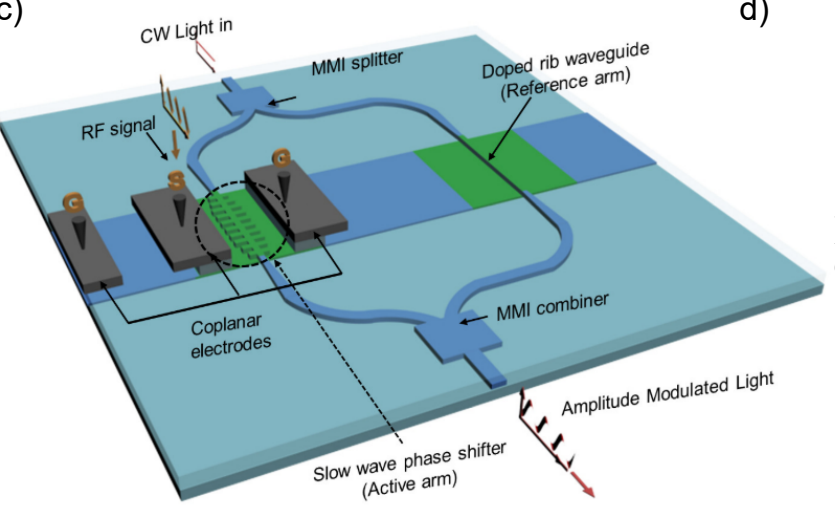

b)

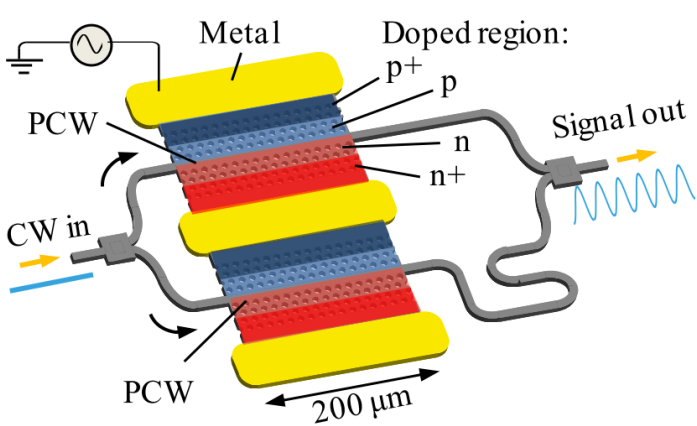

Grating coupler

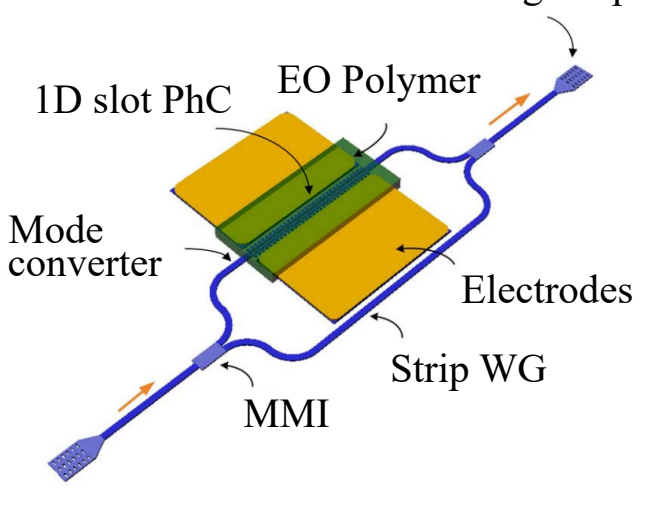

Fig. 5.3. Interferometric configurations as optical modulators and switches including (a) folded waveguides and PhCs either (b) two-dimensional, (c) one-dimensional and (d) slot PhC in the arms of a MZI [121, 130, 30, 134]. 
to reduce losses and increase the extinction ratio must be considered. For example, designing more complex tapered structures as mode converters and step junctions, to minimize both Fresnel reflection and mode field mismatch, respectively. On the other hand, propagation losses are almost negligible and they do not contribute to insertion losses. Besides, note that losses are almost equal between different $\mathrm{PhC}$ design lengths, so that they are not mainly due to this fact, otherwise this would be clearly visible in the transmitted spectra.

In Paper $\mathrm{D}$ we present a switch consisting of a bimodal $\mathrm{PhC}$ waveguide and four input and output ports. The device presents a lower bandwidth than conventional MZIs but higher than ring resonators-based switches. In turn, insertion loss is higher than both MZIs and RRs, due to the highly dispersive periodic structure employed. However, future work in the taper design could minimize losses by improving the coupling efficiency to the higher order odd mode, as it was previously explained. Moreover, further investigation in the $\mathrm{PhC}$ structure could enlarge the bandwidth, for instance designing larger regions of group index contrast and reducing the bimodal length. This could help to increase the free spectral range between consecutive interferences and so the bandwidth. At the end, the proposed switch acts as a modal MZI in single-channel structure, so further developments to offer similar performances than a MZI in terms of bandwidth could be achievable, but in a drastically reduced footprint.

To some extent, the previous work done with bimodal SWG structures has many things in common with the main ideas of the bimodal PhC part. Nevertheless, we believe that the operation principle, development, and findings presented in the latter are clearly

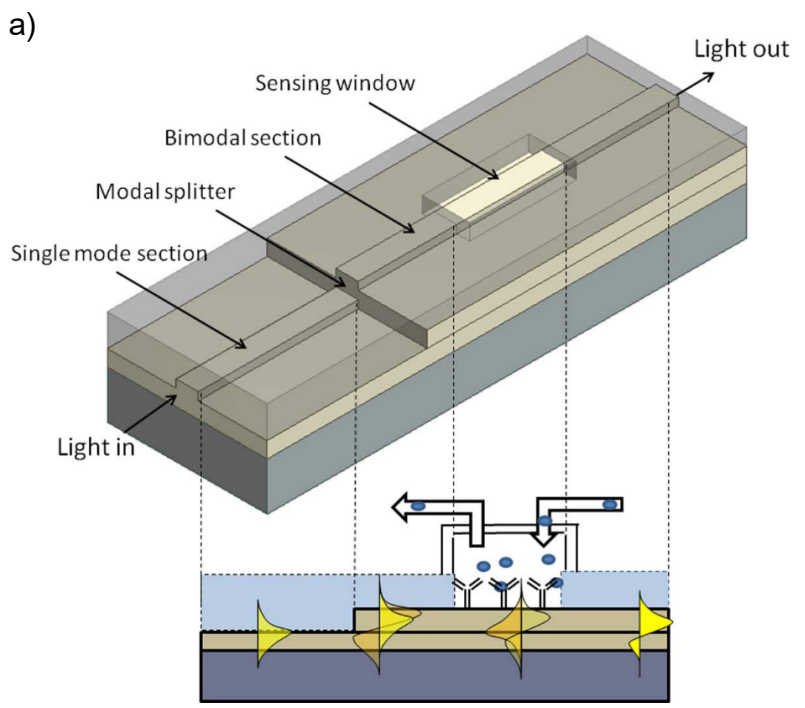

b)
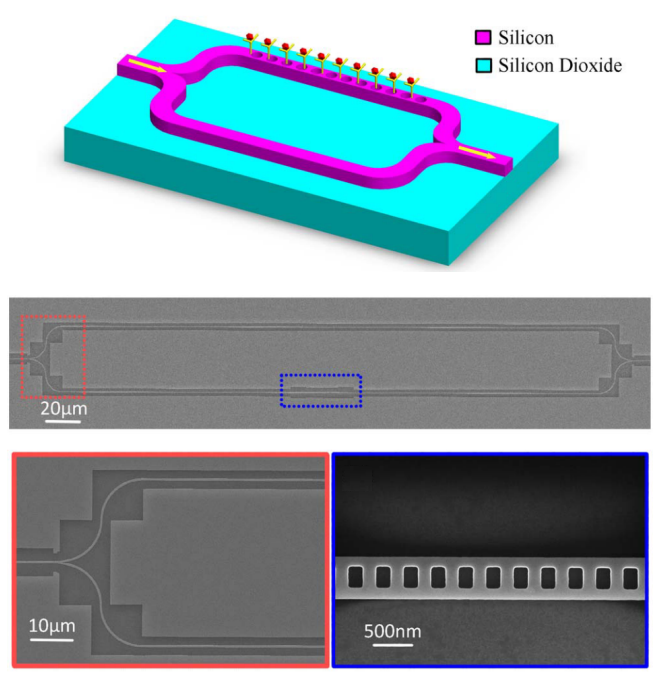

Fig. 5.4. Interferometric refractive index sensors as (a) bimodal waveguides and (b) MZI-based schemes with a 1D PhC in one of the arms [32, 133]. 
different. We designed the periodic structure to support two modes with large group velocity difference in order to enhance the accumulated phase shift for changes in the refractive index of the system, specifically in the higher order mode, in contrast to the dispersion obtained for the fundamental one in the SWG case. To this end, PhCs allow us to engineer the band diagram, and thus to design the desired bimodal slow light regions. Moreover, that is the reason why in the PhC part we provide the sensitivity in terms of radians per refractive index unit, since the phase shift obtained is drastically improved in comparison with the almost negligible phase shift accumulated when dealing with SWG structures. Note that in the case of SWG structures, the group velocity of the fundamental mode is lower than the group velocity of the higher order mode. In contrast, in the case of the photonic crystal the group velocity of the higher order mode is much lower than the group velocity of the fundamental mode. This working condition in photonic crystal structures is emphasized by drastically slowing down the high-order mode. The idea of having a fundamental mode acting as a reference and a higher order mode acting as an active mode for phase shift sensing is exploited in this part, while in the bimodal SWGs both modes interacted with the cladding variations. It would be pointless to sense the accumulated phase shift with SWG structures since it would be very low and the active mode has a higher group velocity than the fundamental one, which would be like designing a MZI with the sensing arm shorter than the reference one, obtaining a not desired operation behavior.

Another important fact that distinguish both parts of the thesis is the field of application of the proposed devices. Bimodal SWG waveguides were mainly focused on sensing devices, since its dispersion effects were already exploited for other passive photonic applications such as polarization controllers and beam splitters. On the other hand, bimodal $\mathrm{PhC}$ can be used in a higher number of active photonic applications, as in the case of the modulators and switches, as a result of the large phase shift accumulated for induced changes in the silicon refractive index. This idea could be extended to many other integrated optical applications as in the case of programmable optics, and logic gates for optical processing, among others. None of the abovementioned applications could be carried out by using the SWG-based bimodal waveguides, since very high changes of the refractive index of the silicon structure would be required to achieve a complete modulation transition, being much higher than in a standard MZI.

\subsection{Main conclusions}

One could say that the reported articles presented in the previous chapters cover a wide variety of topics of very diverse nature. Indeed, during this thesis we did not intended to focus on a specific application, rather on the optical dispersive phenomena that arise 
when dealing with bimodal periodic structures of different kinds. Otherwise, we think that we might have missed some key insights that remain hidden when facing new research for the first time. Hence, the main conclusions achieved in this work come from the intuition of merging different ideas existing in the literature with no apparent relation, in order to explore new potential applications with enhanced operational features.

The research work presented in this dissertation is included within the silicon photonics field, in particular, integrated interferometry. The main goal was to develop bimodal interferometric devices in very compact footprints. To this end, the work carried out combines different periodic structures such as subwavelength gratings and photonic crystals, both aimed at reducing the physical length employed and improving the performance in the interferometry process. Specifically, we explore the resulting effects of engineering dipersive modes in common path interferometers-based on bimodal waveguides, and how these can be exploited for different purposes and applications. In all cases, the designed devices are based on fully etched and all-dielectric structures, which allow the compatibility with foundry processes.

The following list details each of the conclusions obtained:

- In Paper A we theoretically designed a bimodal waveguide-based on a periodic pattern of SWG elements that supports the fundamental even and the first-order odd mode of the TE polarization. By using periodically patterned structures in the near subwavelength region ${ }^{1}$, we achieved a dispersive behavior of the fundamental propagating mode, which produces an almost constant phase shift between both modes as a function of the wavelength. These effects, together with the sensing advantages typical of SWGs, have been exploited to develop an spectral-based refractive index sensor with critical sensitivity regions. The sensor performance is demonstrated to be strongly dependent on the design dimensions since they determine the evolution of the phase shift, and thus the sensitivity of the device. The surface sensing is also studied, providing also outstanding results because of the inherent high-sensitivity performance of the bimodal SWG sensor. Furthermore, by measuring the spectral shift, the design becomes non-dependent on the interferometric length, which benefits the densely integration of multiple devices in a single chip.

- The bimodal SWG sensor has been experimentally demonstrated in Paper B for changes in the bulk refractive index of the cladding. The theoretical predictions have been proved experimentally, providing a detailed comparison with theory and simulations. The influence of the dimensions on the sensitivity has also been analyzed as well as the sensor length non-dependence. As a result, a compact

\footnotetext{
${ }^{1}$ We refer to the near subwavelength to that region close to the Bragg regime.
} 
silicon spectral-based sensor with record-high sensitivity values is experimentally demonstrated in a bimodal SWG waveguide. Compared to the literature, our proposed design provides a higher sensitivity than other spectral-based sensors such as SWG ring resonators, PhCs and BiM interferometers [114, 146, 152] and critically reduces the physical length typically required in uniform BiM waveguides when measuring the phase shift [32].

- Paper C proposed, both theoretically and experimentally, a single-channel interferometer in a one-dimensional $\mathrm{PhC}$ that supports the fundamental and the second-order even modes of the TE polarization. Since both modes are of the same parity, they interfere in the band structure of the $\mathrm{PhC}$, forming an anti-crossing point when the fundamental mode is folded into the first Brillouin zone. For lower wavelengths below this region, the higher order mode becomes slow light, while the fundamental mode presents a non-dispersive behavior. Therein, the higher order mode acts as an active mode while the fundamental one acts as a reference. This bimodal operation enables high-performance interferometry, as large phase shifts occur when a refractive index change is induced without the need of designing very long optical paths. Really compact and all-dielectric interferometers are subsequently demonstrated, as a result of the slow light bimodal interferometry together with the single-channel-based structure and the small lateral size typical of 1D PhCs.

- The bimodal PhC interferometer has been demonstrated in Paper $\mathrm{C}$ for changes in the refractive index of the silicon core and cladding, to demonstrate its efficiency for optical modulation and sensing, respectively. In the first case, the silicon core RI variation has been tested changing the temperature due to the thermo-optic coefficient of the silicon. Phase shifts of $\pi$ have been demonstrated in designs being more than two orders of magnitude smaller than conventional MZIs, and more than one order compared to interferometric schemes including PhCs [23, 21, 30]. Regarding the sensing experiments, the reported sensitivities improve by a factor of 10 traditional MZIs, and around 7.5 uniform bimodal waveguides [120, 32]. Overall, its really compact footprint and straightforward CMOS-compatible design thereby offer clear advantages respect to other interferometers for mass-level production at low cost.

- In Paper D we demonstrate optical switching in a slow-light-enhanced bimodal waveguide for the thermo-optic tunability effect. In this case, the bimodal $\mathrm{PhC}$ waveguide supports the fundamental even and the first-order odd modes of the TE polarization, which adds another degree of freedom in the designing process. By exploiting the different parity of the modes, we achieve a large group index contrast 
between the modes for a wide wavelength operation region. The device has been experimentally tested as a 2x2 switch by using titanium heaters close the photonic structures. As a result, a switching efficiency higher than in a conventional silicon waveguide has been demonstrated, everything integrated in an extremely reduced design area, smaller than the ones used in others PhC-based switches [123, 124]. These results address the ever-growing increase in the traffic of data centers where multiple switches in densely integrated circuits are highly desirable.

- Paper E explored a new method of designing slow-light-enhanced bimodal interferometers for high-performance operation. Here the structures are designed to support the TE fundamental and second-order modes of the even symmetry. A figure of merit that characterizes the group index contrast in a wavelength region is defined and used for principal component analysis. By taking the first two principal components, we reduced the multi-dimensional design space, which allowed the exhaustive mapping of the optimized designs. Consequently, the designs have been investigated for changes in both the core and cladding refractive indices, in order to test their efficiency as modulators and sensors. Moreover, we proposed different geometrical PhC designs to analyze not only the band formation but the light-matter interaction for an specific purpose. Ultra-compact and high-performance interferometers are subsequently presented by using two different simulation methods and with improved properties compared to other similar designs. The results suggest the use of this designing method to engineer novel $\mathrm{PhC}$ structures as bimodal interferometers by considering different performance criteria.

\subsection{Future work}

Given the rise of photonics in the last few decades, this thesis has intended to provide specific solutions for the increasingly high demand of smaller integrated devices. However, we believe there are many aspects that remain open for further investigation in future works, not only improving the existing results, but also seeking for new research directions. Therefore, we herein detail a brief list describing some works that might be considered in future investigations.

- Photonic biosensors have attracted increasing attention from many research groups to develop LoC devices for real-time diagnosis. On this matter, we have been proving the bimodal SWG sensor for the detection of different concentrations of the BSA 
protein using in-flow biofunctionalization processes ${ }^{2}$. However, these tasks are still ongoing and no clear results have yet been obtained. It would be also fruitful to test the bimodal SWG sensor in more complex experiments involving the recognition of some specific biomarkers related with certain diseases.

- The slow light bimodal waveguides have been demonstrated for high performance operation. Nevertheless, it is well known that 2D PhCs are much more efficient providing slow light benefits, which means that higher values of the group index can be obtained for the propagating modes. Taking this into account, it would be very insightful to design slow-light-enhanced bimodal waveguides in $2 \mathrm{D}$ and even 3D PhCs to develop single-channel interferometers with an enhanced performance.

- As in the previous case, PhC bimodal interferometers can be used for biosensing purposes. In addition, since the sensitivity of the sensor is expressed in terms of the phase shift, low-cost interrogation systems could be developed by measuring the sensor response at a given fixed wavelength. Moreover, the bimodal $\mathrm{PhC}$ design could be extrapolated to other CMOS-compatible materials such as silicon nitride in order to operate not only in the infrared, but also in the visible spectrum as in the case of other similar sensing devices to reduce costs and absorption losses in an aqueous environment.

- The bimodal PhC waveguide has been proved for changes in the silicon core refractive index. It could be really interesting to further investigate the performance of the device as a high-speed modulator and switch by improving the heaters design or exploiting plasma dispersion effects in the silicon. Another approach for tuning the bimodal PhC could be using liquid crystal as cladding and silicon electrodes to twist the molecules by applying an electric field ${ }^{3}$. These strong effects, combined with the ultra-compact design of the slow-light-enhanced bimodal interferometers could find potential applications in programmable photonics where large meshes with a high number of optical gates are required.

- The designs proposed by using the dimensionality reduction techniques could be fabricated and compared with the reported configurations. Furthermore, the idea of characterizing the bimodal band structure of a $\mathrm{PhC}$ by a figure of merit could be extrapolated to design other configurations such as reconfigurable interferometers, where we could change the bimodal operation regime by tuning the refractive index of the silicon core.

\footnotetext{
${ }^{2}$ Some results involving biosensing experiments in SWG bimodal waveguides are presented in conferences [Conf. 4] and [Conf. 5] (see Author's merits section).

${ }^{3}$ This task regarding liquid crystal actuation has been partially developed during the research stay at Ghent University.
} 


\subsection{Concluding remarks}

To conclude, I would like to make one final remark on what has motivated me to get throughout all these years and make this thesis.

Art and science have much more in common than it seems. They both are driven by creativity and curiosity of thinking about things that do not exist yet, and perhaps, for being recognized by others. In that sense, a thesis was for me like an empty painting waiting to be sketched. The freedom of creating something was my motivation, just like a craftsman when carving a handmade wooden figure. After all, we are just trying to understand the world we live in, in the most enjoyable manner possible.

"Music is basically like mathematics, you're trying to form patterns, patterns that makes you to understand what is around you and help you get through the next day."

— Thom Yorke 


\section{Author's merits}

\section{Journal articles}

\section{Main contributions:}

[Paper A] Luis Torrijos-Morán and Jaime García-Rupérez, "Single-channel bimodal interferometric sensor using subwavelength structures," Optics Express 27, 8168-8179 (2019).

[Paper B] Luis Torrijos-Morán, Amadeu Griol, and Jaime García-Rupérez, "Experimental study of subwavelength grating bimodal waveguides as ultrasensitive interferometric sensors," Optics Letters 44, 4702-4705 (2019).

[Paper C] Luis Torrijos-Morán, Amadeu Griol, and Jaime García-Rupérez, "Slow light bimodal interferometry in one-dimensional photonic crystal waveguides," Light: Science \& Applications 10, 16 (2021).

[Paper D] Luis Torrijos-Morán, Antoine Brimont, Amadeu Griol, Pablo Sanchis, and Jaime García-Rupérez, "Ultra-compact optical switches using slow light bimodal silicon waveguides," Journal of Lightwave Technology, 39, 3495-3501 (2019).

[Paper E] Luis Torrijos-Morán and Jaime García-Rupérez, "Design of slow-light-enhanced bimodal interferometers using dimensionality reduction techniques," Optics Express 29, 15 (2021).

\section{Other contributions:}

[Paper 6] Raffaele Caroselli, Salvador Ponce-Alcántara, Francisco Prats Quilez, David Martín Sánchez, Luis Torrijos-Morán, Amadeu Griol Barres, Laurent Bellieres, Hanna Bandarenka, Kseniya Girel, Vitaly Bondarenko, and Jaime 
García-Rupérez, "Experimental study of the sensitivity of a porous silicon ring resonator sensor using continuous in-flow measurements," Optics Express 25, 31651-31659 (2017).

[Paper 7] Raffaele Caroselli, David Martín Sánchez, Salvador Ponce Alcántara, Francisco Prats Quilez, Luis Torrijos-Morán, and Jaime García-Rupérez, "Real-time and in-flow sensing using a high sensitivity porous silicon microcavity-based sensor," Sensors 17, 2813 (2017).

[Paper 8] Raffaele Caroselli, David Martín Sánchez, Salvador Ponce Alcántara, Francisco Prats Quilez, Luis Torrijos-Morán, and Jaime García-Rupérez, "Experimental study of a real-time and highly sensitive fiber-optic porous silicon temperature sensing probe," IEEE Sensors Journal 18, 5361-5367 (2018).

[Paper 9] Jad Sabek, Luis Torrijos-Morán, Amadeu Griol, Zeneida Díaz Betancor, María-José Bañuls Polo, Ángel Maquieira, and Jaime García-Rupérez, "Real time monitoring of a UV light-assisted biofunctionalization protocol using a nanophotonic biosensor," Biosensors 9, 6 (2019).

[Paper 10] Jad Sabek, Francisco Javier Díaz-Fernández, Luis Torrijos-Morán, Zeneida Díaz-Betancor, Ángel Maquieira, María-José Bañuls, Elena Pinilla-Cienfuegos, and Jaime García-Rupérez, "Experimental study of an evanescent-field biosensor based on 1D photonic bandgap structures," Beilstein Journal of Nanotechnolgy 10, 967-974 (2019).

\section{Conferences}

\section{Main contributions:}

[Conf. 1] Luis Torrijos-Morán and Jaime García-Rupérez, "High sensitivity optical sensing based on modal interferences in one-dimensional photonic crystals," Poster, 5th International Electronic Conference on Sensors and Applications, Online (2018).

[Conf. 2] Luis Torrijos-Morán and Jaime García-Rupérez, "Modal interferometric sensing in silicon corrugated waveguides," Poster, The European Optical Society Biennial Meeting, Delft, Netherlands (2018).

[Conf. 3] Luis Torrijos-Morán and Jaime García-Rupérez, "Subwavelength grating bimodal waveguide for refractive index sensing," Oral, OSA Advanced Photonics Congress ITh3A.5, San Francisco, USA (2019). 
[Conf. 4] Luis Torrijos-Morán, Amadeu Griol, and Jaime García-Rupérez, "Bimodal waveguide sensors enabled by subwavelength grating structures," Oral, OSA Advanced Photonics Congress, ITu4A. 4, Online (2020).

[Conf. 5] Luis Torrijos-Morán, Paula Martínez-Pérez and Jaime García-Rupérez, "Real-time functionalization and biosensing in subwavelength grating bimodal waveguides," Poster, European Conference on Integrated Optics, Online (2020).

\section{Other contributions:}

[Conf. 6] Raffaele Caroselli, Salvador Ponce-Alcántara, Francisco Prats Quilez, David Martín Sánchez, Luis Torrijos-Morán, Amadeu Griol Barres, Laurent Bellieres, Hanna Bandarenka, Kseniya Girel, Vitaly Bondarenko and Jaime García-Rupérez, "Development and experimental study of a highly sensitive porous silicon ring resonator," 20th European Conference on Integrated Optics, Valencia, Spain (2018).

[Conf. 7] Raffaele Caroselli, Salvador Ponce-Alcántara, David Martín Sánchez, Luis Torrijos-Morán and Jaime García-Rupérez, "Development and experimental study of a porous silicon fiber-optic temperature sensing probe," ImagineNano Bringing together Nanoscience \& Nanotechnology, Bilbao, Spain (2018).

[Conf. 8] Raffaele Caroselli, Salvador Ponce-Alcántara, Francisco Prats Quilez, David Martín Sánchez, Luis Torrijos-Morán, Amadeu Griol Barres, Laurent Bellieres, Hanna Bandarenka, Kseniya Girel, Vitaly Bondarenko and Jaime García-Rupérez, "In-flow and in-continuum refractive index sensing using a highly sensitive porous silicon ring resonator," ImagineNano Bringing togetherNanoscience \& Nanotechnology, Bilbao, Spain (2018).

[Conf. 9] Jad Sabek, Luis Torrijos-Morán, María José Bañuls-Polo, Zeneida Díaz-Betancor, Ángel Maquieira-Catalá, and Jaime García-Rupérez, "In situ functionalization and label free pro-tein detection using a nanophotonic biosensor," XII International Workshop on Sensors and Molecular Recognition, Valencia, Spain (2018).

[Conf. 10] Jad Sabek, Luis Torrijos-Morán, Zeneida Díaz-Betancor, María José Bañuls-Polo, Ángel Maquieira-Catalá and Jaime García-Rupérez, "Live tracking biofunctionalization and label-free protein detection performed by a 
nanophotonic biosensor," 5th International Electronic Conference on Sensors and Applications, Online (2018).

[Conf. 11] Paula Martínez-Pérez, Maribel Gómez-Gómez, Luis Torrijos-Morán, Todora Angelova, Amadeu Griol, Juan Hurtado, Laurent Bellieres, and Jaime García-Rupérez, "1D periodic corrugated waveguides for real-time detection of increasing concentrations of thrombin," OSA Advanced Photonics Congress, ITu4A.8, Online (2020).

[Conf. 12] Jaime García-Rupérez, Luis Torrijos-Morán, Maribel Gómez-Gómez, Paula Martínez-Pérez, and Salvador Ponce-Alcántara, "High performance photonic biosensors based on periodic configurations," SPIE OPTO 11689, Online (2021).

\section{Patents}

[Patent] Luis Torrijos-Morán and Jaime García-Rupérez "Método para maximizar el desfase en una guía bimodal de cristal fotónico," Oficina Española de Patentes y Marcas, Ref. P202031056, Madrid (2020).

\section{Research stays}

[Stay] Ghent University, Ghent, Belgium. Photonics Research Group, Department of Information Technology. Design and simulation of liquid crystal actuation on slow-light-enhanced bimodal waveguides, supervised by Prof. Wim Bogaerts (2020). 


\section{Bibliography}

[1] G. M. Moore, "Cramming more components onto integrated circuits," Electronics, vol. 38, no. 8, pp. 114-117, 1965.

[2] M. Waldrop, “More than moore," Nature, vol. 530, pp. 144-147, 2016.

[3] R. Kirchain and L. Kimerling, "A roadmap for nanophotonics," Nature Photonics, vol. 1, no. 6, pp. 303-305, 2007.

[4] D. Thomson, A. Zilkie, J. E. Bowers, T. Komljenovic, G. T. Reed, L. Vivien, D. Marris-Morini, E. Cassan, L. Virot, J. M. Fédéli, J. M. Hartmann, J. H. Schmid, D. X. Xu, F. Boeuf, P. O’Brien, G. Z. Mashanovich, and M. Nedeljkovic, "Roadmap on silicon photonics,” Journal of Optics, vol. 18, no. 7, pp. 073003-073023, 2016.

[5] M. Haurylau, G. Chen, H. Chen, J. Zhang, N. A. Nelson, D. H. Albonesi, E. G. Friedman, and P. M. Fauchet, "On-chip optical interconnect roadmap: Challenges and critical directions," IEEE Journal on Selected Topics in Quantum Electronics, vol. 12, no. 6, pp. 1699-1704, 2006.

[6] J. Chan, G. Hendry, A. Biberman, and K. Bergman, "Architectural exploration of chip-scale photonic interconnection network designs using physical-layer analysis," Journal of Lightwave Technology, vol. 28, no. 9, pp. 1305-1315, 2010.

[7] H. Subbaraman, X. Xu, A. Hosseini, X. Zhang, Y. Zhang, D. Kwong, and R. T. Chen, "Recent advances in silicon-based passive and active optical interconnects," Optics Express, vol. 23, no. 3, pp. 2487-2511, 2015.

[8] X. Fan and I. M. White, "Optofluidic microsystems for chemical and biological analysis," Nature Photonics, vol. 5, pp. 591-597, 2011.

[9] D. Duval, A. B. González-Guerrero, S. Dante, J. Osmond, R. Monge, L. J. Fernández, K. E. Zinoviev, C. Domínguez, and L. M. Lechuga, "Nanophotonic lab-on-a-chip platforms including novel bimodal interferometers, microfluidics and grating couplers," Lab on a Chip, vol. 12, no. 11, pp. 1987-1994, 2012. 
[10] A. González-Guerrero, J. Maldonado, S. Herranz, and L. M. Lechuga, "Trends in photonic lab-on-chip interferometric biosensors for point-of-care diagnostics," Anal. Methods, vol. 8, no. 48, pp. 8380-8394, 2016.

[11] R. Yu, S. Cheung, Y. Li, K. Okamoto, R. Proietti, Y. Yin, and S. J. B. Yoo, “A scalable silicon photonic chip-scale optical switch for high performance computing systems," Optics Express, vol. 21, no. 23, pp. 9203-9210, 2013.

[12] B. J. Shastri, A. N. Tait, T. F. D. Lima, W. H. P. Pernice, H. Bhaskaran, C. D. Wright, and P. R. Prucnal, "Photonics for artificial intelligence and neuromorphic computing," Nature Photonics, vol. 15, no. February, pp. 102-114, 2021.

[13] A. Rahim, T. Spuesens, R. Baets, and W. Bogaerts, "Open-Access Silicon Photonics: Current Status and Emerging Initiatives," Proceedings of the IEEE, vol. 106, no. 12, pp. 2313-2330, 2018.

[14] R. A. Soref and J. P. Lorenzo, "Single-Crystal Silicon: A New Material For 1-3 And 1-6 Fim Integrated-Optical Components,” Electronics Letters, vol. 21, no. 21, pp. 953-954, 1985.

[15] P. Dumon, W. Bogaerts, V. Wiaux, J. Wouters, S. Beckx, J. Van Campenhout, D. Taillaert, B. Luyssaert, P. Bienstman, D. Van Thourhout, and R. Baets, "Low-loss SOI photonic wires and ring resonators fabricated with deep UV lithography," IEEE Photonics Technology Letters, vol. 16, no. 5, pp. 1328-1330, 2004.

[16] G. T. Reed, G. Mashanovich, F. Y. Gardes, and D. J. Thomson, "Silicon optical modulators," Nature Photonics, vol. 4, no. 8, pp. 518-526, 2010.

[17] W. Bogaerts, R. Baets, P. Dumon, V. Wiaux, S. Beckx, D. Taillaert, B. Luyssaert, J. Van Campenhout, P. Bienstman, and D. Van Thourhout, "Nanophotonic waveguides in silicon-on-insulator fabricated with CMOS technology," Journal of Lightwave Technology, vol. 23, no. 1, pp. 401-412, 2005.

[18] G. V. Treyz, P. G. May, and J. M. Halbout, "Silicon Mach-Zehnder waveguide interferometers based on the plasma dispersion effect," Applied Physics Letters, vol. 59, no. 7, pp. 771-773, 1991.

[19] R. G. Heideman, R. P. H. Kooyman, and J. Greve, "Performance of a highly sensitive optical waveguide Mach-Zehnder interferometer immunosensor," Sensors and Actuators: B. Chemical, vol. 10, no. 3, pp. 209-217, 1993.

[20] J. Van Campenhout, W. M. Green, S. Assefa, and Y. A. Vlasov, "Low-power, $2 \times 2$ silicon electro-optic switch with 110-nm bandwidth for broadband reconfigurable optical networks," Optics Express, vol. 17, no. 26, pp. 24020-24029, 2009. 
[21] W. M. Green, M. J. Rooks, L. Sekaric, and Y. A. Vlasov, "Ultra-compact, low RF power, $10 \mathrm{~Gb} / \mathrm{s}$ silicon Mach-Zehnder modulator," Optics Express, vol. 15, no. 25, pp. 17106-17113, 2007.

[22] D. J. Thomson, F. Y. Gardes, J. M. Fedeli, S. Zlatanovic, Y. Hu, B. P. P. Kuo, E. Myslivets, N. Alic, S. Radic, G. Z. Mashanovich, and G. T. Reed, "50-Gb/s silicon optical modulator,” IEEE Photonics Technology Letters, vol. 24, no. 4, pp. 234-236, 2012.

[23] L. Liao, D. Samara-Rubio, M. Morse, A. Liu, D. Hodge, D. Rubin, U. D. Keil, and T. Franck, "High speed silicon Mach-Zehnder modulator," Optics Express, vol. 13, no. 8, pp. 3129-3135, 2005.

[24] B. Sepúlveda, J. Sánchez Del Río, M. Moreno, F. J. Blanco, K. Mayora, C. Domínguez, and L. M. Lechuga, "Optical biosensor microsystems based on the integration of highly sensitive Mach-Zehnder interferometer devices," Journal of Optics A: Pure and Applied Optics, vol. 8, no. 7, p. S561, 2006.

[25] R. Sumi, R. K. Gupta, N. DasGupta, and B. K. Das, "Ultra-Broadband Add-Drop Filter/Switch Circuit Using Subwavelength Grating Waveguides," IEEE Journal of Selected Topics in Quantum Electronics, vol. 25, no. 3, pp. 1-11, 2018.

[26] R. Halir, P. J. Bock, P. Cheben, A. Ortega-Moñux, C. Alonso-Ramos, J. H. Schmid, J. Lapointe, D. X. Xu, J. G. Wangüemert-Pérez, Í. Molina-Fernández, and S. Janz, "Waveguide sub-wavelength structures: A review of principles and applications," Laser and Photonics Reviews, vol. 9, no. 1, pp. 25-49, 2015.

[27] P. Cheben, R. Halir, J. H. Schmid, H. A. Atwater, and D. R. Smith, "Subwavelength integrated photonics," Nature, vol. 560, no. 7720, pp. 565-572, 2018.

[28] J. D. Joannopoulos, P. R. Villeneuve, and S. Fan, "Photonic crystals: Putting a new twist on light," Nature, vol. 386, no. 6621, pp. 143-149, 1997.

[29] E. A. Camargo, H. M. Chong, and R. M. De La Rue, "Highly compact asymmetric Mach-Zehnder device based on channel guides in a two-dimensional photonic crystal," Applied Optics, vol. 45, no. 25, pp. 6507-6510, 2006.

[30] A. Brimont, D. J. Thomson, P. Sanchis, J. Herrera, F. Gardes, J. M. Fedeli, G. T. Reed, and J. Martí, "High speed silicon electro-optical modulators enhanced via slow light propagation," Optics Express, vol. 19, no. 21, pp. 20876-20885, 2011.

[31] R. Levy and S. Ruschin, "Design of a single-channel modal interferometer waveguide sensor," IEEE Sensors Journal, vol. 9, no. 2, pp. 146-153, 2009. 
[32] K. E. Zinoviev, A. B. González-Guerrero, C. Domínguez, and L. M. Lechuga, "Integrated bimodal waveguide interferometric biosensor for label-free analysis," Journal of Lightwave Technology, vol. 29, no. 13, pp. 1926-1930, 2011.

[33] C. S. Huertas, D. Fariña, and L. M. Lechuga, "Direct and label-free quantification of micro-RNA-181a at attomolar level in complex media using a nanophotonic biosensor," ACS Sensors, vol. 1, no. 6, pp. 748-756, 2016.

[34] C. S. Huertas, S. Domínguez-Zotes, and L. M. Lechuga, "Analysis of alternative splicing events for cancer diagnosis using a multiplexing nanophotonic biosensor," Scientific Reports, vol. 7, pp. 1-8, 2017.

[35] C. S. Huertas, O. Calvo-Lozano, A. Mitchell, and L. M. Lechuga, "Advanced Evanescent-Wave Optical Biosensors for the Detection of Nucleic Acids: An Analytic Perspective," Frontiers in Chemistry, vol. 7, no. October, pp. 1-25, 2019.

[36] T. Maiman, "Stimulated optical radiation in ruby," Nature, vol. 187, pp. 493-494, 1960.

[37] B. Jalali and S. Fathpour, "Silicon photonics," Journal of Lightwave Technology, vol. 24, no. 12, pp. 189-205, 2006.

[38] C. Gunn, "CMOS photonics for high-speed interconnects," IEEE Micro, vol. 26, pp. 58-66, 2006.

[39] M. J. Weber, Handbook of optical materials. CRC Press, 2003.

[40] D. Marcuse, Theory of Dielectric Optical Waveguides. New York: Academic, 1991.

[41] G. Brambilla, V. Finazzi, and D. J. Richardson, "Ultra-low-loss optical fiber nanotapers," Optics Express, vol. 12, no. 10, pp. 2258-2263, 2004.

[42] P. K. Tien, "Integrated optics and new wave phenomena in optical waveguides," Reviews of Modern Physics, vol. 49, no. 2, pp. 361-420, 1977.

[43] R. Soref, "The past, present, and future of silicon photonics," IEEE Journal on Selected Topics in Quantum Electronics, vol. 12, no. 6, pp. 1678-1687, 2006.

[44] M. Paniccia, "Integrating silicon photonics," Nature Photonics, vol. 4, no. 8, pp. 498-499, 2010.

[45] G. Lifante, Integrated Photonics: Fundamentals. John Wiley \& Sons, New York, 2003.

[46] G. Reed and A. Knights, Silicon Photonics - An Introduction. John Wiley \& Sons, Chichester, 2004. 
[47] H. Hertz, “Ueber Strahlen elektrischer Kraft,” Wiedemanns Ann, vol. 36, p. 769, 1888.

[48] L. Rayleigh, "On the influence of obstacles arranged in rectangular order upon the properties of a medium," The London, Edinburgh, and Dublin Philosophical Magazine and Journal of Science, vol. 34, no. 211, pp. 481-502, 1892.

[49] S. Rytov, "Electromagnetic properties of a finely stratified medium," JETP, Soviet Physics, vol. 2, no. 3, pp. 466-475, 1956.

[50] C. G. Bernhard and W. H. Miller, "A Corneal Nipple Pattern in Insect Compound Eyes," Acta Physiologica Scandinavica, vol. 56, no. 3-4, pp. 385-386, 1962.

[51] P. Clapham and M. Hutley, "Reduction of Lens Reflexion by the "Moth Eye" Principle," Nature, vol. 244, pp. 281-282, 1973.

[52] P. Yeh, "A new optical model for wire grid polarizers," Optics Communications, vol. 26, no. 3, pp. 289-292, 1978.

[53] C. Yang and P. Yeh, "Artificial uniaxial and biaxial dielectrics with the use of photoinduced gratings," Journal of Applied Physics, vol. 81, no. 1, pp. 23-29, 1997.

[54] M. C. Huang, Y. Zhou, and C. J. Chang-Hasnain, "A surface-emitting laser incorporating a high-index-contrast subwavelength grating," Nature Photonics, vol. 1, no. 2, pp. 119-122, 2007.

[55] D. A. Bruggeman, "Berechnung verschiedener physikalischer Konstanten von heterogenen Substanzen. III. Die elastischen Konstanten der quasiisotropen Mischkörper aus isotropen Substanzen," Annalen der Physik, vol. 421, no. 2, pp. 160-178, 1935.

[56] L. Brekhovskikh, Waves in layered media. Elsevier, 1980.

[57] G. Floquet, "Sur les équations différentielles linéaires à coefficients périodiques," vol. 12, pp. 47-88, 1883.

[58] E. Peral and J. Capmany, "Generalized bloch wave analysis for fiber and waveguide gratings," Journal of Lightwave Technology, vol. 15, no. 8, pp. 1295-1302, 1997.

[59] R. Kashyap, Fiber Bragg Gratings. San Diego, CA: Academic Press, 1999.

[60] J. D. Joannopoulos, S. G. Johnson, J. N. Winn, and R. D. Meade, Photonic crystals: Molding the flow of light. Princeton Univ. Press, 2007.

[61] N. Bloembergen, Nonlinear Optics. New York: World Scientific, 1965. 
[62] A. R. McGurn and A. A. Maradudin, "Photonic band structures of two- and three-dimensional periodic metal or semiconductor arrays," Physical Review B, vol. 48, pp. 17576-17579, 1993.

[63] V. Kuzmiak, A. A. Maradudin, and F. Pincemin, "Photonic band structures of two-dimensional systems containing metallic components," Physical Review B, vol. 50, no. 23, pp. 16835-16844, 1994.

[64] C. Kittel, Introduction to Solid State Physics. New York: Wiley \& Sons, 1996.

[65] J. Mathews and R. Walker, Mathematical Methods of Physics. California Institute of Technology, 1964.

[66] S. Johnson and J. Joannopoulos, "Block-iterative frequency-domain methods for Maxwell's equations in a planewave basis," Optics Express, vol. 8, no. 3, pp. 173-190, 2001.

[67] T. Baba, "Slow light in photonic crystals," Nature Photonics, vol. 2, no. 8, pp. 465-473, 2008.

[68] R. D. Meade, A. Devenyi, J. D. Joannopoulos, O. L. Alerhand, D. A. Smith, and K. Kash, "Novel applications of photonic band gap materials: Low-loss bends and high Q cavities," Journal of Applied Physics, vol. 75, no. 9, pp. 4753-4755, 1994.

[69] S. Fan, J. D. Joannopoulos, J. N. Winn, A. Devenyi, J. C. Chen, and R. D. Meade, "Guided and defect modes in periodic dielectric waveguides," Journal of the Optical Society of America B, vol. 12, no. 7, pp. 1267-127, 1995.

[70] D. M. Atkin, P. S. J. Russell, T. A. Birks, and P. J. Roberts, "Photonic band structure of guided bloch modes in high index films fully etched through with periodic microstructure," Journal of Modern Optics, vol. 43, no. 5, pp. 1035-1053, 1996.

[71] M. Françon, Optical Interferometry. New York: Academic Press, 1966.

[72] M. Vaughan, The Fabry-Perot Interferometer. Bristol: CRC Press, 1989.

[73] L. Yuan, J. Yang, Z. Liu, and J. Sun, "In-fiber integrated Michelson interferometer," Optics Letters, vol. 31, no. 18, pp. 2692-2694, 2006.

[74] L. Zehnder, "Ein neuer Interferenzrefraktor," Instrumentenkunde, vol. 11, pp. 275-285, 1891.

[75] E. Mach, "Uever einen Interferenzrefraktor," Instrumentenkunde, vol. 12, pp. 89-94, 1892. 
[76] W. Lukosz and C. Stamm, "Integrated optical interferometer as relative humidity sensor and differential refractometer," Sensors and Actuators: A. Physical, vol. 25, no. 1-3, pp. 185-188, 1991.

[77] W. Lukosz, C. Stamm, H. R. Moser, R. Ryf, and J. Dübendorfer, "Difference interferometer with new phase-measurement method as integrated-optical refractometer, humidity sensor and biosensor," Sensors and Actuators, B: Chemical, vol. 39, no. 1-3, pp. 316-323, 1997.

[78] R. Levy and S. Ruschin, "Critical sensitivity in hetero-modal interferometric sensor using spectral interrogation.," Optics express, vol. 16, no. 25, pp. 20516-20521, 2008.

[79] K. P. Zetie, S. F. Adams, and R. M. Tocknell, "How does a Mach-Zehnder interferometer work?," Physics Education, vol. 35, no. 1, pp. 46-48, 2000.

[80] P. Kozma, F. Kehl, E. Ehrentreich-Förster, C. Stamm, and F. F. Bier, "Integrated planar optical waveguide interferometer biosensors: A comparative review," Biosensors and Bioelectronics, vol. 58, pp. 287-307, 2014.

[81] N. Shaw, W. Stewart, J. Heaton, and D. Wight, "Optical slow-wave resonant modulation in electro-optic GaAs/AlGaAs modulators," Electronics letters, vol. 35, pp. 1557-1558, 1999.

[82] R. Levy, S. Ruschin, and D. Goldring, "Critical sensitivity effect in an interferometer sensor.," Optics letters, vol. 34, no. 19, pp. 3023-3025, 2009.

[83] M. Notomi, K. Yamada, A. Shinya, J. Takahashi, C. Takahashi, and I. Yokohama, "Extremely large group-velocity dispersion of line-defect waveguides in photonic crystal slabs," Physical Review Letters, vol. 87, no. 25, pp. 253902-253904, 2001.

[84] S. Olivier, M. Rattier, H. Benisty, C. Weisbuch, C. J. Smith, R. M. De La Rue, T. F. Krauss, U. Oesterle, and R. Houdré, "Mini-stopbands of a one-dimensional system: The channel waveguide in a two-dimensional photonic crystal," Physical Review B, vol. 63, no. 11, pp. 1-4, 2001.

[85] T. F. Krauss, "Slow light in photonic crystal waveguides," Journal of Physics D: Applied Physics, vol. 40, no. 9, pp. 2666-2670, 2007.

[86] T. F. Krauss, "Why do we need slow light?," Nature Photonics, vol. 2, no. 8, pp. 448-450, 2008.

[87] P. Lalanne, S. Astilean, P. Chavel, E. Cambril, and H. Launois, "Design and fabrication of blazed binary diffractive elements with sampling periods smaller than the structural cutoff," Journal of the Optical Society of America A, vol. 16, no. 5, pp. 1143-1156, 1999. 
[88] J. N. Mait and D. W. Prather, Selected Papers on Subwavelength Diffractive Optics. Bellingham, WA: SPIE Optical Engineering Press, 2001.

[89] N. Bloembergen and A. J. Sievers, "Nonlinear Optical Properties of Periodic Laminar Structures," Applied Physics Letters, vol. 17, no. 11, pp. 483-486, 1970.

[90] J. P. Van Der Ziel, "Phase-matched harmonic generation in a laminar structure with wave propagation in the plane of the layers," Applied Physics Letters, vol. 26, no. 2, pp. 60-61, 1975.

[91] P. Cheben, D.-X. Xu, S. Janz, and A. Densmore, "Subwavelength waveguide grating for mode conversion and light coupling in integrated optics," Optics Express, vol. 14, no. 11, pp. 4695-4702, 2006.

[92] P. Cheben, S. Janz, D. X. Xu, B. Lamontagne, A. Delâge, and S. Tanev, "A broad-band waveguide grating coupler with a subwavelength grating mirror," IEEE Photonics Technology Letters, vol. 18, no. 1, pp. 13-15, 2006.

[93] J. H. Schmid, P. Cheben, S. Janz, J. Lapointe, E. Post, A. Delâge, A. Densmore, B. Lamontagne, P. Waldron, and D. X. Xu, "Subwavelength grating structures in silicon-on-insulatorwaveguides," Advances in Optical Technologies, 2008.

[94] P. J. Bock, P. Cheben, J. H. Schmid, J. Lapointe, A. Delâge, S. Janz, G. C. Aers, D.-X. Xu, A. Densmore, and T. J. Hall, "Subwavelength grating periodic structures in silicon-on-insulator: a new type of microphotonic waveguide," Optics Express, vol. 18, no. 19, pp. 20251-20262, 2010.

[95] R. Halir, P. Cheben, S. Janz, D.-X. Xu, Í. Molina-Fernández, and J. G. Wangüemert-Pérez, "Waveguide grating coupler with subwavelength microstructures,” Optics Letters, vol. 34, no. 9, pp. 1408-1410, 2009.

[96] R. Halir, P. Cheben, J. H. Schmid, R. Ma, D. Bedard, S. Janz, D.-X. Xu, A. Densmore, J. Lapointe, and Í. Molina-Fernández, "Continuously apodized fiber-to-chip surface grating coupler with refractive index engineered subwavelength structure," Optics Letters, vol. 35, no. 19, pp. 3243-3245, 2010.

[97] P. Cheben, P. J. Bock, J. H. Schmid, J. Lapointe, S. Janz, D.-X. Xu, A. Densmore, A. Delâge, B. Lamontagne, and T. J. Hall, "Refractive index engineering with subwavelength gratings for efficient microphotonic couplers and planar waveguide multiplexers," Optics Letters, vol. 35, no. 15, pp. 2526-2528, 2010.

[98] P. Cheben, J. H. Schmid, S. Wang, D.-X. Xu, M. Vachon, S. Janz, J. Lapointe, Y. Painchaud, and M.-J. Picard, "Broadband polarization independent 
nanophotonic coupler for silicon waveguides with ultra-high efficiency," Optics Express, vol. 23, no. 17, pp. 22553-22563, 2015.

[99] I. Glesk, P. J. Bock, P. Cheben, J. H. Schmid, J. Lapointe, and S. Janz, “All-optical switching using nonlinear subwavelength Mach-Zehnder on silicon," Optics Express, vol. 19, no. 15, pp. 14031-14039, 2011.

[100] A. Ortega-Moñux, L. Zavargo-Peche, A. Maese-Novo, I. Molina-Fernández, R. Halir, J. G. Wangüemert-Pérez, P. Cheben, and J. H. Schmid, "High-performance multimode interference coupler in silicon waveguides with subwavelength structures," IEEE Photonics Technology Letters, vol. 23, no. 19, pp. 1406-1408, 2011.

[101] R. Halir, P. Cheben, J. M. Luque-González, J. D. Sarmiento-Merenguel, J. H. Schmid, G. Wangüemert-Pérez, D. X. Xu, S. Wang, A. Ortega-Moñux, and Í. Molina-Fernández, "Ultra-broadband nanophotonic beamsplitter using an anisotropic sub-wavelength metamaterial," Laser and Photonics Reviews, vol. 10, no. 6, pp. 1039-1046, 2016.

[102] J. M. Luque-González, A. Herrero-Bermello, A. Ortega-Moñux, Í. Molina-Fernández, A. V. Velasco, P. Cheben, J. H. Schmid, S. Wang, and R. Halir, "Tilted subwavelength gratings: controlling anisotropy in metamaterial nanophotonic waveguides," Optics Letters, vol. 43, no. 19, pp. 4691-4694, 2018.

[103] A. Herrero-Bermello, A. Dias-Ponte, J. M. Luque-González, A. Ortega-Moñux, A. V. Velasco, P. Cheben, and R. Halir, "Experimental demonstration of metamaterial anisotropy engineering for broadband on-chip polarization beam splitting," Optics Express, vol. 28, no. 11, p. 16385, 2020.

[104] D. González-Andrade, J. G. Wangüemert-Pérez, A. Velasco, A. Ortega-Moñux, A. Herrero-Bermello, I. Molina-Fernández, R. Halir, and P. Cheben, "Ultra-Broadband Mode Converter and Multiplexer Based on Sub-Wavelength Structures,” IEEE Photonics Journal, vol. 10, no. 2, pp. 1-10, 2018.

[105] S. Jahani, S. Kim, J. Atkinson, J. C. Wirth, F. Kalhor, A. A. Noman, W. D. Newman, P. Shekhar, K. Han, V. Van, R. G. Decorby, L. Chrostowski, M. Qi, and Z. Jacob, "Controlling evanescent waves using silicon photonic all-dielectric metamaterials for dense integration," Nature Communications, vol. 9, no. 1, pp. 1893-1902, 2018.

[106] J. M. Luque-gonzález, R. Halir, J. G. Wangüemert-pérez, J. H. Schmid, P. Cheben, Í. Molina-fernández, and A. Ortega-moñux, "An Ultracompact GRIN-Lens-Based Spot Size Converter using Subwavelength Grating Metamaterials," Laser \& Photonics Reviews, vol. 13, pp. 1-7, 2019. 
[107] J. Gonzalo Wangüemert-Pérez, P. Cheben, A. Ortega-Moñux, C. Alonso-Ramos, D. Pérez-Galacho, R. Halir, I. Molina-Fernández, D.-X. Xu, and J. H. Schmid, "Evanescent field waveguide sensing with subwavelength grating structures in silicon-on-insulator," Optics Letters, vol. 39, no. 15, pp. 4442-4445, 2014.

[108] J. G. Wangüemert-Pérez, A. Hadij-ElHouati, A. Sánchez-Postigo, J. Leuermann, D. X. Xu, P. Cheben, A. Ortega-Moñux, R. Halir, and Í. Molina-Fernández, "Subwavelength structures for silicon photonics biosensing," Optics and Laser Technology, vol. 109, pp. 437-448, 2019.

[109] V. Donzella, A. Sherwali, J. Flueckiger, S. M. Grist, S. T. Fard, and L. Chrostowski, "Design and fabrication of SOI micro-ring resonators based on sub-wavelength grating waveguides," Optics Express, vol. 23, no. 4, pp. 4791-4803, 2015.

[110] J. Flueckiger, S. Schmidt, V. Donzella, A. Sherwali, D. M. Ratner, L. Chrostowski, and K. C. Cheung, "Sub-wavelength grating for enhanced ring resonator biosensor," Optics Express, vol. 24, no. 14, pp. 15672-15686, 2016.

[111] H. Yan, L. Huang, X. Xu, S. Chakravarty, N. Tang, H. Tian, and R. T. Chen, "Unique surface sensing property and enhanced sensitivity in microring resonator biosensors based on subwavelength grating waveguides," Optics Express, vol. 24, no. 26, pp. 29724-29733, 2016.

[112] H. Tian, H. Yan, N. Tang, R. T. Chen, S. Chakravarty, L. Huang, X. Xu, H. Yan, X. Xu, S. Chakravarty, N. Tang, H. Tian, and R. T. Chen, "Improving the detection limit for on-chip photonic sensors based on subwavelength grating racetrack resonators," Optics Express, vol. 25, no. 9, pp. 10527-10535, 2017.

[113] Z. Zhang, G. I. Ng, T. Hu, H. Qiu, X. Guo, W. Wang, M. S. Rouifed, C. Liu, J. Sia, J. Zhou, C. G. Littlejohns, G. T. Reed, and H. Wang, "Mid-infrared sensor based on a suspended microracetrack resonator with lateral subwavelength-grating metamaterial cladding," IEEE Photonics Journal, vol. 10, no. 2, pp. 1-8, 2018.

[114] E. Luan, H. Yun, L. Laplatine, Y. Dattner, D. M. Ratner, K. C. Cheung, and L. Chrostowski, "Enhanced Sensitivity of Subwavelength Multibox Waveguide Microring Resonator Label-Free Biosensors," IEEE Journal of Selected Topics in Quantum Electronics, vol. 25, no. 3, pp. 1-11, 2019.

[115] J. R. Bickford, P. S. Cho, M. E. Farrell, E. L. Holthoff, and P. M. Pellegrino, “The Investigation of Subwavelength Grating Waveguides for Photonic Integrated Circuit Based Sensor Applications," IEEE Journal of Selected Topics in Quantum Electronics, vol. 25, no. 3, pp. 1-10, 2019. 
[116] M. A. Butt, S. N. Khonina, S. N. Khonina, N. L. Kazanskiy, and N. L. Kazanskiy, "A highly sensitive design of subwavelength grating double-slot waveguide microring resonator," Laser Physics Letters, vol. 17, no. 7, pp. 076201-076209, 2020.

[117] C. K. Tang and G. T. Reed, "Highly efficient optical phase modulator in SOI waveguides," Electronics Letters, vol. 31, no. 6, pp. 451-452, 1995.

[118] R. A. Soref and S. Member, "Silicon-Based Optoelectronics," Proceedings of the IEEE, vol. 81, no. 12, pp. 1687-1706, 1993.

[119] P. V. Lambeck, "Integrated opto-chemical sensors," Sensors and Actuators: B. Chemical, vol. 8, no. 1, pp. 103-116, 1992.

[120] A. Calle, A. Llobera, and C. Dom, “An integrated optical interferometric nanodevice based on silicon technology for biosensor applications A Abad, A Montoya and L M Lechuga," Online, vol. 14, pp. 907-912, 2003.

[121] A. Densmore, S. Janz, R. Ma, J. H. Schmid, D.-X. Xu, A. Delâge, J. Lapointe, M. Vachon, and P. Cheben, "Compact and low power thermo-optic switch using folded silicon waveguides," Optics Express, vol. 17, no. 13, pp. 10457-10465, 2009.

[122] D. Dai, Z. Wang, J. Peters, and J. E. Bowers, "Compact polarization beam splitter using an asymmetrical Mach-Zehnder interferometer based on silicon-on-insulator waveguides," IEEE Photonics Technology Letters, vol. 24, no. 8, pp. 673-675, 2012.

[123] E. A. Camargo, H. M. H. Chong, and R. M. De La Rue, "2D Photonic crystal thermo-optic switch based on AlGaAs/GaAs epitaxial structure," Optics Express, vol. 12, no. 4, pp. 588-592, 2004.

[124] H. Nakamura, Y. Sugimoto, K. Kanamoto, and N. Ikeda, "Ultra-fast photonic crystal/quantum dot all-optical switch for future photonic networks," Optics Express, vol. 12, no. 26, pp. 6606-6614, 2004.

[125] Y. Jiang, W. Jiang, L. Gu, X. Chen, and R. T. Chen, "80-Micron Interaction Length Silicon Photonic Crystal Waveguide Modulator," Applied Physics Letters, vol. 87, no. 22, pp. 1-3, 2005.

[126] L. Gu, W. Jiang, X. Chen, L. Wang, and R. T. Chen, "High speed silicon photonic crystal waveguide modulator for low voltage operation," Applied Physics Letters, vol. 90, no. 7, pp. 10-13, 2007.

[127] J.-M. Brosi, C. Koos, L. C. Andreani, M. Waldow, J. Leuthold, and W. Freude, "High-speed low-voltage electro-optic modulator with a polymer-infiltrated silicon photonic crystal waveguide," Optics Express, vol. 16, no. 6, pp. 4177-4191, 2008. 
[128] D. M. Beggs, T. P. White, L. O'Faolain, and T. F. Krauss, “Ultracompact and low-power optical switch based on silicon photonic crystals," Optics Letters, vol. 33, no. 2, pp. 147-149, 2008.

[129] L. O'Faolain, D. M. Beggs, T. P. White, T. Kampfrath, K. Kuipers, and T. F. Krauss, "Compact optical switches and modulators based on dispersion engineered photonic crystals," IEEE Photonics Journal, vol. 2, no. 3, pp. 404-414, 2010.

[130] H. C. Nguyen, Y. Sakai, M. Shinkawa, N. Ishikura, and T. Baba, "10 Gb/s operation of photonic crystal silicon optical modulators,” Optics Express, vol. 19, no. 14, pp. 13000-13007, 2011.

[131] H. C. Nguyen, S. Hashimoto, M. Shinkawa, and T. Baba, "Compact and fast photonic crystal silicon optical modulators,” Optics Express, vol. 20, no. 20, pp. 22465-22474, 2012.

[132] A. Brimont, P. Sanchis, and J. Martí, "Strong electro-optical modulation enhancement in a slow wave corrugated waveguide," Optics Express, vol. 17, no. 11, pp. 9204-9211, 2009.

[133] K. Qin, S. Hu, S. T. Retterer, I. I. Kravchenko, and S. M. Weiss, "Slow light Mach-Zehnder interferometer as label-free biosensor with scalable sensitivity," Optics Letters, vol. 41, no. 4, pp. 753-756, 2016.

[134] H. Yan, X. Xu, C.-J. Chung, H. Subbaraman, Z. Pan, S. Chakravarty, and R. T. Chen, "One-dimensional photonic crystal slot waveguide for silicon-organic hybrid electro-optic modulators,” Optics Letters, vol. 41, no. 23, pp. 5466-5469, 2016.

[135] M. Scalora, R. J. Flynn, S. B. Reinhardt, R. L. Fork, M. J. Bloemer, M. D. Tocci, C. M. Bowden, H. S. Ledbetter, J. M. Bendickson, J. P. Dowling, and R. P. Leavitt, "Ultrashort pulse propagation at the photonic band edge: Large tunable group delay with minimal distortion and loss," Physical Review E, vol. 54, no. 2, pp. 1078-1081, 1996.

[136] T. Y. Yun and K. Chang, "Uniplanar one-dimensional photonic-bandgap structures and resonators," IEEE Transactions on Microwave Theory and Techniques, vol. 49, no. 3, pp. 549-553, 2001.

[137] W. C. Hopman, P. Pottier, D. Yudistira, J. Van Lith, P. V. Lambeck, R. M. De La Rue, A. Driessen, H. J. Hoekstra, and R. M. De Ridder, "Quasi-one-dimensional photonic crystal as a compact building-block for refractometric optical sensors," IEEE Journal on Selected Topics in Quantum Electronics, vol. 11, no. 1, pp. 11-15, 2005. 
[138] M. L. Povinelli, S. G. Johnson, and J. D. Joannopoulos, "Slow-light, band-edge waveguides for tunable time delays," Optics Express, vol. 13, no. 18, pp. 7145-7159, 2005.

[139] E. G. Turitsyna and S. Webb, "Fabrication of low-loss photonic wires in silicon-on-insulator using hydrogen silsesquioxane electron-beam resist," Electronics letters, vol. 41, no. 2, pp. 40-41, 2005.

[140] R. B. Hwang, "Negative group velocity and anomalous transmission in a one-dimensionally periodic waveguide," IEEE Transactions on Antennas and Propagation, vol. 54, no. 2, pp. 755-760, 2006.

[141] Y. Ma, B. Dong, B. Li, J. Wei, Y. Chang, C. P. Ho, and C. Lee, "Mid-Infrared Slow Light Engineering and Tuning in 1-D Grating Waveguide," IEEE Journal of Selected Topics in Quantum Electronics, vol. 24, no. 6, pp. 1-8, 2018.

[142] J. Sabek, F. J. Díaz-Fernández, L. Torrijos-Morán, Z. Díaz-Betancor, Á. Maquieira, M.-J. Bañuls, E. Pinilla-Cienfuegos, and J. García-Rupérez, "Experimental study of an evanescent-field biosensor based on 1D photonic bandgap structures," Beilstein Journal of Nanotechnology, vol. 10, pp. 967-974, 2019.

[143] M. Centini, C. Sibilia, M. Scalora, G. D’Aguanno, M. Bertolotti, M. J. Bloemer, C. M. Bowden, and I. Nefedov, "Dispersive properties of finite, one-dimensional photonic band gap structures: Applications to nonlinear quadratic interactions," Physical Review E, vol. 60, no. 4, pp. 4891-4898, 1999.

[144] M. Notomi, "Manipulating light with strongly modulated photonic crystals," Reports on Progress in Physics, vol. 73, no. 9, pp. 096501-096558, 2010.

[145] R. Won, "Slow light now and then," Nature Photonics, vol. 2, no. 8, pp. 454-455, 2008.

[146] A. Di Falco, L. O'Faolain, and T. F. Krauss, "Chemical sensing in slotted photonic crystal heterostructure cavities," Applied Physics Letters, vol. 94, no. 6, pp. 92-95, 2009.

[147] A. Figotin and I. Vitebskiy, "Slow wave phenomena in photonic crystals," Laser and Photonics Reviews, vol. 5, no. 2, pp. 201-213, 2011.

[148] K. E. Zinoviev, C. Dominguez, and L. M. Lechuga, "Silicon Nitride Bimodal Waveguides for High Sensitivity Biosensors," European Conference on Integrated Optics (ECIO), 2010.

[149] R. Bruck and R. Hainberger, "Sensitivity and design of grating-assisted bimodal interferometers for integrated optical biosensing," Optics Express, vol. 22, no. 26, pp. 32344-32352, 2014. 
[150] Y. Liang, M. Zhao, Z. Wu, and G. Morthier, "Investigation of grating-assisted trimodal interferometer biosensors based on a polymer platform," Sensors (Switzerland), vol. 18, no. 5, pp. 1502-1511, 2018.

[151] B. Chocarro-Ruiz, S. Herranz, A. Fernández Gavela, J. Sanchís, M. Farré, M. P. Marco, and L. M. Lechuga, "Interferometric nanoimmunosensor for label-free and real-time monitoring of Irgarol 1051 in seawater," Biosensors and Bioelectronics, vol. 117, no. March, pp. 47-52, 2018.

[152] Y. Liang, M. Zhao, Z. Wu, and G. Morthier, "Bimodal Waveguide Interferometer RI Sensor Fabricated on Low-Cost Polymer Platform," IEEE Photonics Journal, vol. 11, no. 2, pp. 1-8, 2019.

[153] K. Uchiyamada, K. Okubo, K. Asakawa, Y. Kamon, Y. Kitayama, T. Takeuchi, and H. Suzuki, "Perforated Bimodal Interferometric Biosensor for Affinity Sensing," Advanced Materials Technologies, vol. 4, no. 9, pp. 1-7, 2019.

[154] A. Maese-Novo, J. G. Wangüemert-Pérez, D.-X. Xu, A. Ortega-Moñux, S. Janz, P. Cheben, J. H. Schmid, I. Molina-Fernández, and R. Halir, "Colorless directional coupler with dispersion engineered sub-wavelength structure," Optics Express, vol. 20, no. 12, pp. 13470-13477, 2012.

[155] X. Xu, C. J. Chung, Z. Pan, H. Yan, and R. T. Chen, "Periodic waveguide structures for on-chip modulation and sensing," Japanese Journal of Applied Physics, vol. 57, no. 8, pp. 08PA041-08PA049, 2018.

[156] M. Odeh, K. Twayana, K. Sloyan, J. E. Villegas, S. Chandran, and M. S. Dahlem, "Mode Sensitivity Analysis of Subwavelength Grating Slot Waveguides," IEEE Photonics Journal, vol. 11, no. 5, pp. 1-10, 2019. 


\section{List of figures}

1.1 Electronic equipment shrinkage in size as a function of time. Approximately, every decade a new class of machines emerged in agreement with Moore's law prediction [2]. . . . . . . . . . . . . . 2

2.1 Light rays propagating through a planar optical waveguide with three different media with a refractive index of the core $n_{1}$, and upper and lower

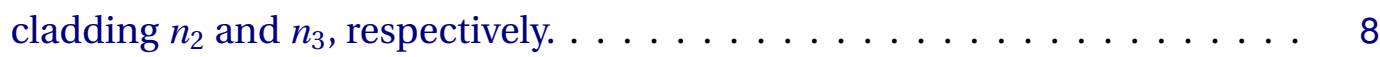

2.2 Rectangular waveguides consisting of stripe, rib and buried structures on a planar substrate, from left to right, respectively. The refractive index of the core (blue colored material) is designed to be higher than the one of the cladding (grey colored material)

2.3 Hertz's experiment on electromagnetic wave propagation through subwavelength wire grid polarizers. a) The electromagnetic wave pass through the polarizing filter when the wires are perpendicular to the dipoles and b) transmission is blocked when they are parallel. . . . . . . . . . 15

2.4 Finely stratified structure consisting of two layers of electric permittivity $\epsilon_{1,2}$ and magnetic permeability $\mu_{1,2}$, respectively, repeated along the $z$ axis with

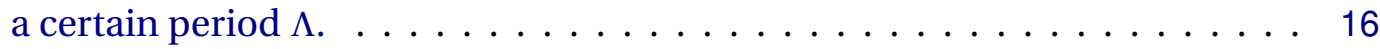

2.5 Electromagnetic components distribution for a propagating wave along the layers with $\mathbf{E}$ vector parallel to the interface. . . . . . . . . . . 16

2.6 Electromagnetic components distribution for a propagating wave along the layers with $\mathbf{H}$ vector parallel to the interface. . . . . . . . . . . . . . . 20

2.7 Electromagnetic components distribution for a propagating wave normal

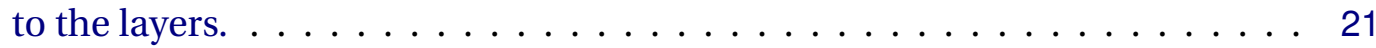

2.8 Schematic of a periodic dielectric waveguide based on an array of subwavelength elements on a substrate. . . . . . . . . . . . . . . . 24 
2.9 Light behaviour in a periodic dielectric waveguide [27]: schematic dispersion diagram and field distribution of its corresponding region, left and right part, respectively. . . . . . . . . . . . . . . .

2.10 Top view of a multimode subwavelength waveguide sketch of a certain width $w$ with the representation of the two first lower order modes [27]. . . . 27

2.11 Sketch of periodic structures as one-dimensional (1D), two-dimensional (2D) and three-dimensional (3D) photonic crystals. The colors represent different materials with their respective dielectric constants. . . . . . . . . .

2.12 Square lattice of a two-dimensional photonic crystal. On the left hand side the real space is represented and on the right hand side the reciprocal lattice with its corresponding Brillouin zone $[60] . \ldots \ldots \ldots$. . . . . . .

2.13 Photonic band structure of a two-dimensional rectangular array of dielectric columns. The red lines represent the TE polarization modes, while the blue ones represent the TM polarization modes. The left inset represents the Brillouin zone with the irreducible area shaded in light yellow. The right inset schematically shows the real space with the dielectric distribution of two dielectric materials represented in different colors [60]. .

2.14 One-dimensional photonic crystal consisting of a multilayer structure and its corresponding band diagram. Three different examples are depicted: on the left graph, both layers present the same dielectric constant $\epsilon_{1}=\epsilon_{2}=13$, in the middle low-index contrast layers of $\epsilon_{1}=13$ and $\epsilon_{2}=12$ and on the right high-index contrast layers of $\epsilon_{1}=13$ and $\epsilon_{2}=1[60] \ldots \ldots \ldots 35$

2.15 Periodic dielectric waveguides examples consisting of one-dimensional periodic patterns in the propagation direction. . . . . . . . . . . .

2.16 Band diagrams of an uniform and one-dimensional periodic waveguide for the TM polarization, left and right graphs, respectively [60]. . . . . . . . .

2.17 Integrated interferometer examples. A Mach-Zehnder configuration is shown on the left hand side, while a bimodal waveguide is depicted on the right hand side. . . . . . . . . . . . . . . . .

2.18 Comparison between the uniform and the subwavelength bimodal waveguides under a static cladding and for a given variation in its refractive index $\Delta n_{c}$, solid and dashed lines, respectively. From upper to lower figures, the dispersion relations, phase shifts and transmitted spectra are shown. . . 
2.19 Band structure representation of one-dimensional photonic crystals. The example on the left side hand schematically shows the behavior of the bands for a single mode configuration. On the right side hand, the band structure of a bimodal configuration is depicted. The solid lines show the bands of the periodic structure, while the dashed lines represent the hypothetical bands of a uniform non-periodic waveguide of similar

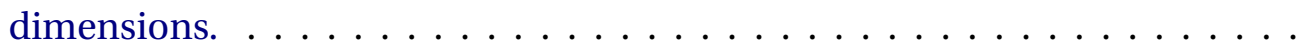

A.1 Schematic representation of the proposed SWG bimodal sensor where ' $\Lambda$ ' is the period, ' $D C$ ' the duty cycle of the SWG elements, ' $w$ ' the width of the SWG elements, ' $h$ ' the height and ' $w_{s}$ ' the width of the input and output

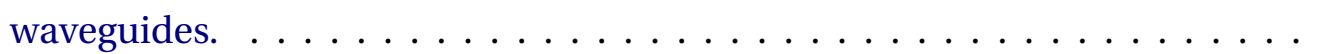

A.2 Dispersion relations and phase shift of the even and odd TE-like modes of a SWG bimodal structure of period $\Lambda=290 \mathrm{~nm}, D C=50 \%, w=1400 \mathrm{~nm}$ and $h=220 \mathrm{~nm}$, under an aqueous environment as cladding $(n=1.36) \ldots \ldots$.

A.3 Phase shift between both modes of the SWG bimodal structure for different duty cycles. Numerical wavelength shift as a function of the cladding RI for different duty cycles at $1665 \mathrm{~nm}$. Bulk sensitivity comparison between the numerical and the semi-analytic calculations obtained using Eq. (3) at an operation wavelength of $1665 \mathrm{~nm} . \ldots \ldots \ldots$

A.4 Surface sensitivity as a function of the layer thickness $\rho$ for the SWG bimodal sensor in an aqueous environment for different duty cycles. Surface sensitivity as a function of wavelength for different layer thicknesses and a duty cycle of $60 \%$. The layer thickness goes from $10 \mathrm{~nm}$

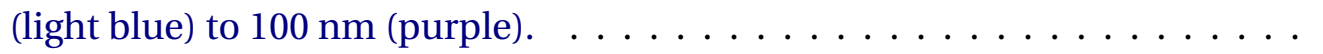

A.5 Electric field magnitude distribution of a periodic cell of the SWG structure in different planes for even and odd modes. Mode profile of the $x$ component of the electric field for both modes in the $x$ - axis at $y=0$ and $z=0$. Transmission spectra for a $50 \%$ duty cycle SWG bimodal structure as a function of the displacement ' $d$ ' of a single mode waveguide of width $w_{s}=450 \mathrm{~nm}$ at the input and output. . . . . . . . . . . . . 61 
A.6 Spectral shift as a function of cladding RI variations for $50 \%$ and $60 \%$ duty cycles. Transmission spectra for $50 \%$ duty cycle (top) and $60 \%$ duty cycle (bottom) at different RI scenarios. $x$ - component of the electric field at $y=$ 0 slice. The upper contour map represents maximum transmission for a wavelength exhibiting constructive interference. The lower contour map depicts minimum transmission for a wavelength where a spectral dip is located (i.e., destructive interference) . . . . . . . . . . . . . . . . .

A.7 Comparison between the sensitivity results obtained in MPB and CST for the SWG sensor having a duty cycle of $50 \%$ and $60 \%$. Semi-analytical curves (solid and dashed lines) represent the theoretical bulk sensitivity as a function of wavelength obtained using MPB and Eq. (3). Diamonds and circle markers show the sensitivity of certain spectral fringes in the spectrum obtained from the FDTD simulations. . . . . . . . . . . . . . . . . 64

B.1 Sketch of the proposed bimodal SWG sensor and its design parameters. Dispersion diagram of even and odd modes. Phase shift " $\Delta \phi=L *\left(\beta_{\text {even }}-\right.$ $\beta_{\text {odd }}$ )" between the even and odd modes as a function of wavelength. Electric field energy density of the TE even and odd modes excited in the SWG bimodal region. SEM image of the interface between the SWG bimodal waveguide and the single-mode access waveguide. . . . . . . . .

B.2 Experimental normalized transmission spectra with respect to a reference waveguide, obtained for a bimodal SWG sensor. Experimental comparison between the numerical simulations and the absolute wavelength shifts of each spectral dip for a refractive index change of $3.2 \times 10^{-3}$ RIU as a function

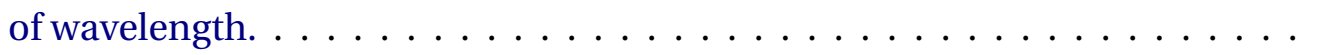

B.3 Time evolution of the spectral dips located at $1580 \mathrm{~nm}$ with. Representation of the absolute wavelength shift of the spectral dips under different RI variations. The linear fitting represents the bulk sensitivity experimentally obtained for both configurations. . . . . . . . . . . . . . . .

B.4 Color map representing the numerical derivative of the phase shift with respect to the cladding RI (upper graph) and wavelength (lower graph). Comparison between the simulated sensitivity obtained using Eq. (1) (red curve) and the experimental results considering a different number of periods $N$ (colored bars). . . . . . . . . . . . . . . . . . 78 
C.1 Design and simulation of the 1D PhC. 3D sketch of the proposed design composed of two single-mode waveguides at the input/output ports and a rectangular taper between the uniform and periodic bimodal parts. Dispersion diagram of the 1D PhC showing the first three $x$-even parity bands for TE-like polarization. Real part of the electric field $x$-component for the fundamental and higher-order modes in the $x z$ plane for $y=0 \ldots . .92$

C.2 Optimization of the band structure. Second and third bands for different transversal element lengths $w_{e}$. Trade-off between the group velocity difference between both modes (in blue) and the bimodal bandwidth (in orange) as a function of $w_{e}$. Ratio between the output and input power, calculated as the squared cosine of the phase shift as a function of the

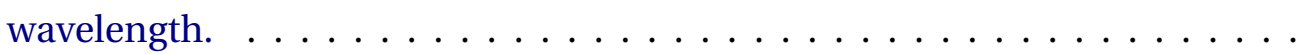

C.3 Simulation of the bimodal behaviour. Transmission spectra for a bimodal PhC interferometer with and without a rectangular taper. Modal excitation obtained from the amplitude difference between constructive and destructive interference in the spectrum for different taper lengths. Electric field absolute value for the $x z$ plane and $y=0$ at the output interface. FFT amplitude of the electric field $x$-component along the propagation direction $z$ for different wavelengths in the bimodal region. . .

C.4 Experimental measurements of slow light bimodal behaviour. Scanning electron microscopy (SEM) image of the fabricated silicon structures without a silica cover layer. Normalized experimental transmitted spectra for $N=200$ and $N=400$ periods under a silica cover. Group index experimentally obtained from the maxima and minima interference points and with the simulated fundamental mode group index as a reference. . . .

C.5 Optical response for changes in the silicon temperature. Experimental phase shift obtained in the fabricated bimodal $1 \mathrm{D} \mathrm{PhC} \mathrm{interferometer} \mathrm{as}$ a function of the wavelength. Evolution of the phase shift measured for a linear increment in the chip temperature. The upper graph represents a close-up view of the normalized transmission spectrum for the $N=$ 200 configuration (a bimodal 1D PhC length of $74 \mu \mathrm{m}$ ). The lower graph represents a close-up view of the normalized transmission spectrum for the $N=400$ configuration (a bimodal 1D PhC length of $148 \mu \mathrm{m}$ ) . . . . . . . 101 
C.6 Optical response for changes in the cladding refractive index. Normalized experimental spectra obtained for the $N=400$ configuration fabricated without a silica cover layer when the structure is covered with different ethanol concentrations in DIW dilutions. Phase shift measurements for both the $N=200$ and $N=400$ configurations as a function of the linear increments in the cladding RI. RI sensitivity experimentally obtained for $N=200$ and $N=400$ bimodal 1D PhC sensors as the slope of the linear fitting for different changes in the cladding RI. . . . . . . . . . . . . . . . 103

D.1 Device schematic of the proposed 2x2 optical switch design, composed of two input and output single-mode silicon waveguides, a bimodal $1 \mathrm{D} \mathrm{PhC}$ structure and a rectangular taper or transition between these two parts, placed both at the input and output interfaces. . . . . . . . . . . 116

D.2 Band structure of the 1D PhC periodic unit cell for the TE-like polarized bands. Detailed band structure and group index for both even and odd modes in the bimodal region, upper and lower graph, respectively. Absolute value of the electric field $x$-component at $y=0 \mathrm{~nm}$ plane for the third even parity band and the second odd parity band. 3D-FDTD simulation of the real part $x$-component electric field propagation at $y=0 \mathrm{~nm}$ plane. . . . . 117

D.3 Trade-off between the insertion losses and the group index of the odd parity mode as a function of the transversal element width $w_{e}$. Simulated $\pi$ length for different silicon refractive index increments at a common wavelength of

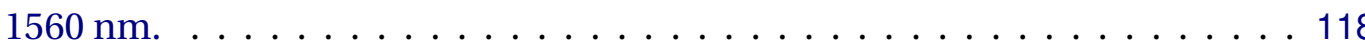

D.4 Optical microscope image of the fabricated chip with the input and output grating couplers, the titanium (Ti) heaters and lateral pads. SEM image of the optical switch with the input and output single mode waveguides and detailed 1D PhC structure in silicon . . . . . . . . . . . . . . . . . . 120

D.5 Normalized transmission spectra of the fabricated optical 2x2 switches with a transversal element width $w_{e 1}$ and $w_{e 2}$ with a silica cover as upper cladding. Experimental group index calculated from the spectra minima and maxima peaks as a function of wavelength for both $w_{e 1}$ and $w_{e 2}$ designs. 122

D.6 Normalized transmission spectra for different voltages and for both designs of $w_{e 1}=1600 \mathrm{~nm}$ with a device length of $74 \mu \mathrm{m}$ and $w_{e 1}=1700 \mathrm{~nm}$. Phase shift and optical power as a function of the electrical power applied, left and right graph, respectively. . . . . . . . . . . . . . . . . . . . . . . . . 124 
E.1 Bimodal band structure representation. The first three bands are shown as well as the contribution of the fundamental and high-order modes. Third band considered to perform the interferometry and group index as a function of wavelength. The gray shaded area represents the FoM. . . . . 134

E.2 Corrugated waveguide design optimization. Sketch of the complete slow-light-enhanced interferometer. Colormap of the figure of merit PCA, for the first and second principal components of the collection of good designs. Degradation of the Figure of merit for a width variation of +-10 nm. Upper cladding RI sensitivity normalized to a $1 \mathrm{~cm}$ length at $\lambda_{0}$ wavelength. Length required for a $\pi$ phase shift at $\lambda_{0}$ wavelength. . . . . . . . . .

E.3 Double hole waveguide design optimization. Sketch of the complete slowlight-enhanced interferometer. Colormap of the two principal components of the figure of merit, degradation for deviations of $+-10 \mathrm{~nm} . \ldots \ldots$

E.4 Multi box waveguide design optimization. Sketch of the complete slow-light-enhanced interferometer with the unit cell dimensions of the 1D PhC. Colormap of the two principal components of the figure of merit, degradation for deviations of $+-10 \mathrm{~nm}$, normalized sensitivity to $1 \mathrm{~cm}$ and $\pi$ length. . . . . . . . . . . . . . . . . . . . . . . . . 139

E.5 First three TE-like polarized bands of the band diagram for the optimized designs in the PCA. Real part of the $x$-component field in the corrugated waveguide design for the fundamental mode at $k_{z}=0.3752 \pi / a$ and the higher order mode at $k_{z}=0.4752 \pi / a$. Field distribution in the double hole waveguide and multibox waveguide designs. . . . . . . . . . . . . . . 141

E.6 Comparison of the optimized designs in FDTD simulations. Phase shift of the first constructive interference for changes in cladding and in the silicon refractive index. Transmission spectra of the slow light bimodal interferometers with $\mathrm{N}=100$ periods and for different cladding RI. Transmission spectra for all PCA optimized designs with a length $L_{\pi} \ldots \ldots 143$

5.1 SWG designs based on multimode operation for different photonic applications such as a directional coupler, beam splitter and our proposed bimodal refractive index sensor. . . . . . . . . . . . . . . . . . 152

5.2 SWG based refractive index sensors consisting of ring resonators slot waveguides and MZI configurations. . . . . . . . . . . . . . . . 153

5.3 Interferometric configurations as optical modulators and switches including folded waveguides and PhCs either two-dimensional, one-dimensional and slot PhC in the arms of a MZI . . . . . . . . . . . . . 155 
5.4 Interferometric refractive index sensors as bimodal waveguides and MZI-based schemes with a 1D PhC in one of the arms. . . . . . . . . . . . . 156 


\section{List of acronyms}

\begin{tabular}{ll} 
1D & One-Dimensional \\
2D & Two-Dimensional \\
AU & Three-Dimensional \\
BiM & Arbitrary Units \\
BZ & Bimodal \\
CMOS & Brillouin Zone \\
CST & Complementary Metal-Oxide-Semiconductor \\
CW & Continuous Wave \\
DC & Duty Cycle \\
DIW & Deionized Water \\
EtOH & Ethanol \\
FDTD & Finite Difference Time Domain \\
FEDER & European Regional Development Fund \\
\hline FoM & Figure of Merit \\
\hline Fourier Transform \\
\hline Thology
\end{tabular}


FSR Free Spectral Range

FWHM Full Width at Half Maximum

HSQ Hydrogen Silsesquioxane

IEEE Institute of Electrical and Electronics Engineers

ITO Indium Tin Oxide

LoC Lab on a Chip

MIT Massachusetts Institute of Technology

MMI Multimode Interferometers

MPB MIT Photonics Bands

MZI Mach-Zehnder Interferometer

OSA Optical Society of America

PBG Photonic Bandgap

PCA Principal Component Analysis

PhC Photonic Crystal

PHC PHOCNOSIS

PhD Doctor of Philosophy

PIC Photonic Integrated Circuit

PWE Plain Wave Expansion

RI Refractive Index

RIU Refractive Index Units

ROI Region Of Interest

RR Ring Resonator 
SEM Scanning Electron Microscope

SOI Silicon On Insulator

SWG Subwavelength Grating

TE Transverse Electric

TI Titanium

TIR Total Internal Reflection

TM Transverse Magnetic

UPV Universitàt Politècnica de València 
